\title{
MiRNAs and tumor suppressors form a gene regulatory network to protect multiciliogenesis
}

\section{Dissertation}

for the award of the degree

"Doctor rerum naturalium (Dr. rer. nat.)"

Georg-August-Universität Göttingen

\author{
within the Molecular Medicine Study Program \\ of the Georg-August University School of Science (GAUSS)
}

submitted by

Merit Wildung

from Gifhorn, Germany

Göttingen, 2018 


\section{Thesis Committee}

Dr. Muriel Lizé, Research Group Molecular \& Experimental Pneumology, Clinic for Cardiology and Pneumology, University Medical Center Göttingen

Prof. Dr. Matthias Dobbelstein, Institute of Molecular Oncology, University Medical Center Göttingen

PD Dr. Halyna Shcherbata, Research Group Gene Expression and Signaling, Max-PlanckInstitute for Biophysical Chemistry, Göttingen

\section{Members of the Examination Board}

Dr. Muriel Lizé, Research Group Molecular \& Experimental Pneumology, Clinic for Cardiology and Pneumology, University Medical Center Göttingen

Prof. Dr. Matthias Dobbelstein, Institute of Molecular Oncology, University Medical Center Göttingen

\section{Further members of the Examination Board}

PD Dr. Halyna Shcherbata, Research Group Gene Expression and Signaling, Max-PlanckInstitute for Biophysical Chemistry, Göttingen

Prof. Dr. Frauke Alves, Research Group Molecular Biology of Neuronal Signals, MaxPlanck-Institute for Experimental Medicine, Göttingen

Prof. Dr. Gregor Bucher, Department of evolutionary developmental genetics, University Medical Center Göttingen

Prof. Dr. Luis Pardo, Research Group Oncophysiology, Max-Planck-Institute for Experimental Medicine, Göttingen 


\section{AFFIDAVIT}

I hereby declare that my doctoral thesis entitled "MiRNAs and tumor suppressors form a gene regulatory network to protect multiciliogenesis" has been written independently with no other sources and aids than quoted.

Merit Wildung

Göttingen, $19^{\text {th }}$ October 2018 


\section{ACKNOWLEDGEMENTS}

I would like to thank my supervisor Dr. Muriel Lizé for giving me the opportunity to do my Ph.D. in her group - for her guidance, scientific advice and support during this time. I have learned a lot throughout my time with her and I am grateful for this experience.

I want to thank my thesis advisory committee members Prof. Dr. Matthias Dobbelstein and Dr. Halyna Shcherbata for their interesting and helpful discussions during our annual meetings. Especially, I would like to thank Prof. Dr. Matthias Dobbelstein for giving me additional scientific input and for the helpful advice throughout my PhD. Moreover, I am grateful to the extended committee members - Prof. Dr. Frauke Alves, Prof. Dr. Gregor Bucher, and Prof. Dr. Luis Pardo for taking the time to read and evaluate my thesis.

Special thanks to the Göttingen Graduate School for Neurosciences, Biophysics, and Molecular Biosciences (GGNB) and the Ph.D. program Molecular Medicine for accepting me as member of this program and for offering interesting courses and retreats. In particular, I want to thank Steffen Burkhardt for his support towards the end of my PhD.

Many thanks to all my external collaborators who contributed to this work: Dr. Dietmar Riedel for the electron microscopy data, Dr. Christian Herr for lung function analysis and NTHi-treatment, Dr. Mihai Alevra for his assistance on the mucociliary clearance assay, Ph.D. Haotian Zhou and Dr. Ann-Kathrin Guenther for brain analysis, Naira Movsisyan for the primary cilia disassembly assay, Hataitip Tasena for patient data analysis, Dr. Sharen Provoost for the DEP-treated samples, Dr. Tabea Menchen and Dr. Julia Wallmeier for showing me the cilia beat frequency analysis technique.

I want to thank all my coworkers from the pneumology group: Conny, Evrim, Jan, Larisa, Maren, Sabine, and Tilman. Special thanks to Tilman for starting and helping me with the TAp73 project, to Maren for being my great motivated Master student, to Conny, Sabine, and Larisa for their help for both projects, especially during the revision of our paper. I thank Prof. Dr. Stefan Andreas, the head of the pneumology, for his scientific discussions e.g. during the $U 4$ meetings.

Heartfelt thanks to all the members of the Molecular Oncology for adopting me. I truly had a great time working in the big lab, you have created a genuinely nice and happy environment. Sharing experiences with you guys have helped me a lot, not only scientifically, but also emotionally.

I want to thank Amke, Andrea, Anusha, Jan, Josephine, and Maren for proof reading this thesis and for their incredibly helpful corrections.

Finally, I am very grateful to my family and Jörg for their constant loving support, always encouraging me and being there for me. I am also very grateful to my roommates and friends for listening to me and distracting me occasionally.

My sincere thanks go to the Deutsche Forschungsgesellschaft (DFG) and the GGNB (Bridging fund stipend) for their financial support. 


\section{TABLE OF CONTENTS}

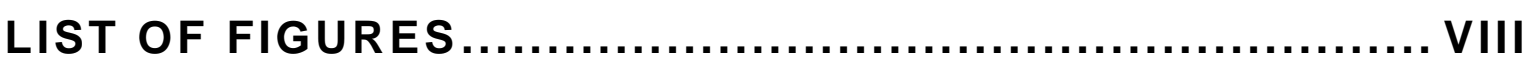

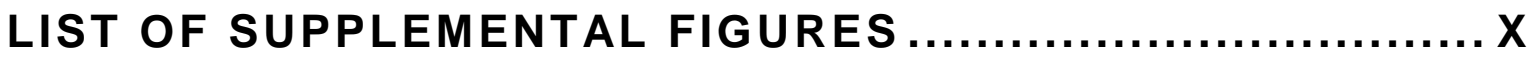

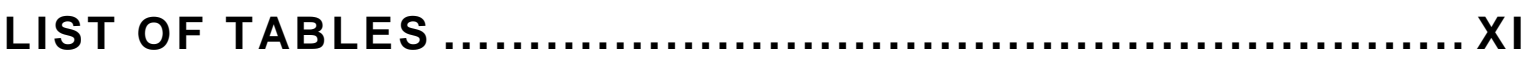

LIST OF SUPPLEMENTAL TABLES $\ldots \ldots \ldots \ldots \ldots \ldots \ldots \ldots \ldots \ldots \ldots \ldots \ldots \ldots \ldots$

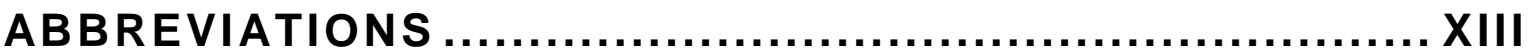

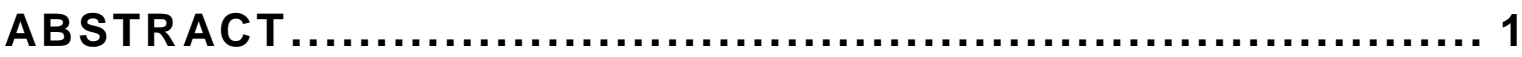

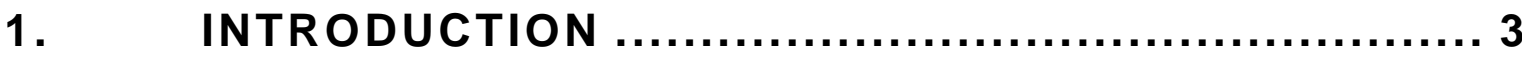

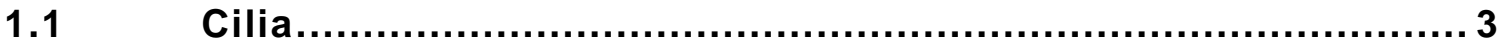

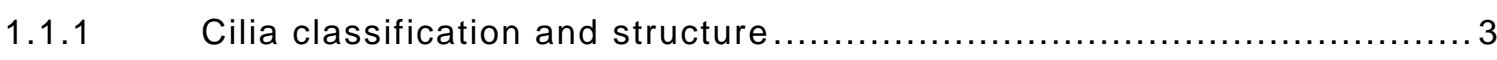

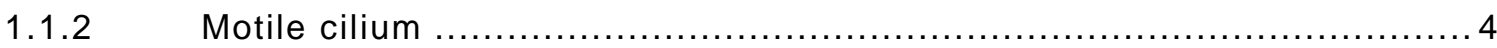

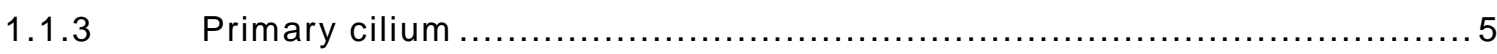

1.2 Location and function of motile cilia in diverse tissues...........5

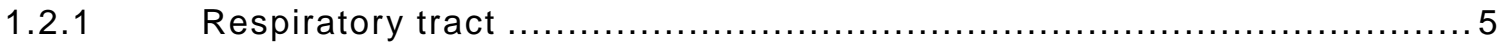

1.2.1.1 Respiratory epithelial cells and their function ..............................

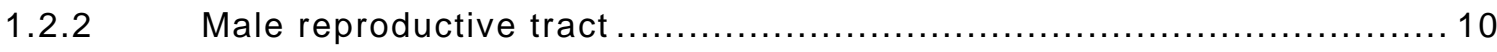

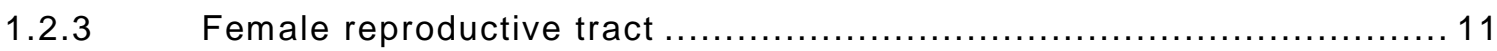

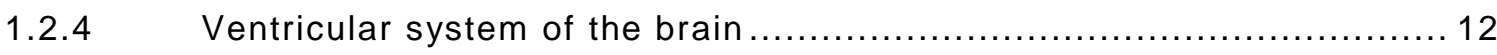

1.3 Regulation of multiciliogenesis - from centrioles to cilia....... 13

1.3.1 Centriole/basal body biogenesis ........................................... 13

1.3.2 Ciliary assembly is mediated by intraflagellar transport .................... 14

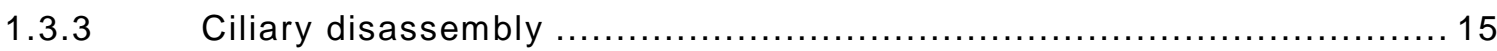

1.3.3.1 Cell cycle-associated functions of Aurora family members................. 17

1.3.4 Transcriptional regulation of multiciliated cell differentiation ….......... 17

1.3.4.1 Master regulators of multiciliogenesis - GEMC1, MCIDAS, and E2Fs ... 18

1.3.4.2 P73 - a novel central regulator of multiciliogenesis .......................... 19

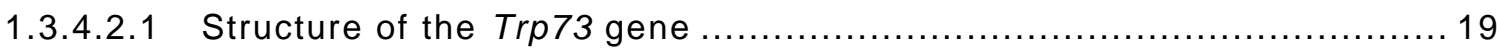

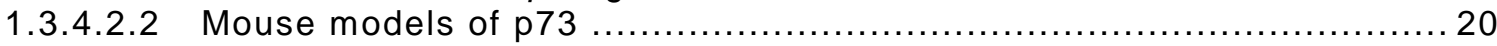

1.3.4.3 Downstream effectors of multiciliogenesis .................................... 22

1.3.5 Post-transcriptional regulation of multiciliated cell differentiation .........23

1.3.5.1 miRNA biogenesis and mechanism of action ................................ 23

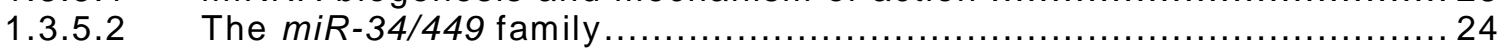

1.4 Diseases associated with motile cilia dysfunction ................ 26

1.4.1 Primary ciliary dyskinesia - Inherited cilia dysfunction ..................... 26

1.4.2 Chronic obstructive pulmonary disease - Acquired cilia dysfunction .....26

1.5 Airway epithelial regeneration - a process impaired in

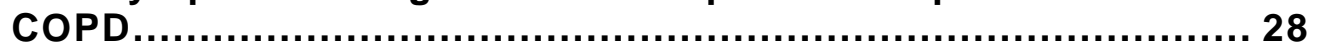

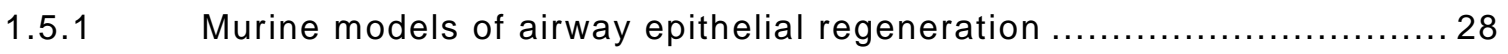




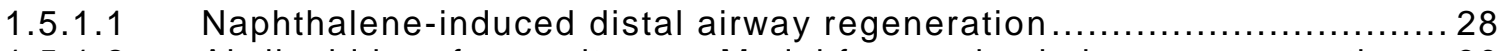

1.5.1.2 Air-liquid interface cultures - Model for proximal airway regeneration... 30

1.6 Preliminary results - TAp73 is a central transcriptional regulator of airway multiciliogenesis

2. OBJECTIVES

3.

METHODS

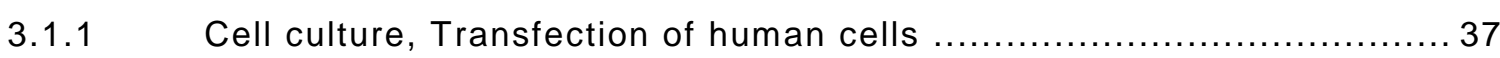

3.1.2 Analysis of primary cilia assembly and disassembly ................... 37

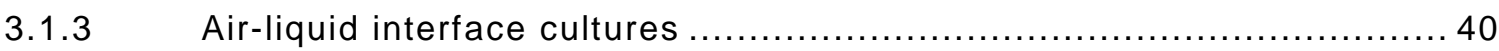

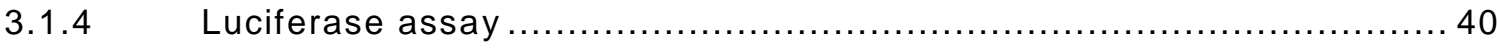

3.2 Mouse Work, Mouse Models ..................................... 41

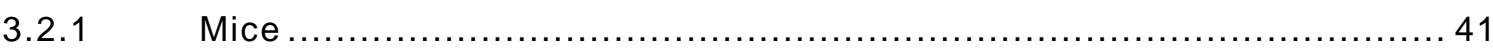

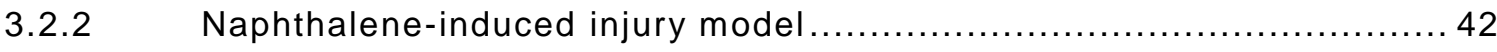

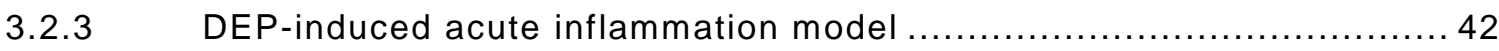

3.2.4 NTHi-induced chronic inflammation model ...........................42

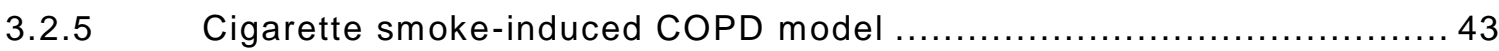

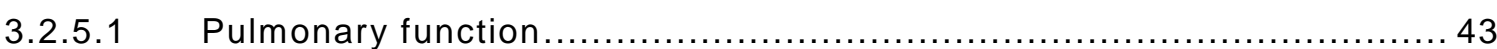

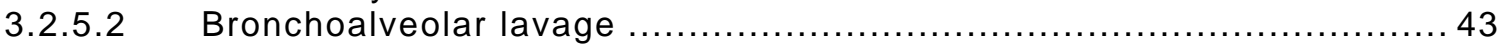

3.2.5.3 Stereology .... 43

3.2.6 Tissue sample preparation for protein and RNA analyses ..............44

$3.3 \quad$ Human samples ........................................................... 44

3.3.1 Human samples of the male reproductive duct........................ 44

3.3.2 Human COPD bronchial biopsy samples, mRNA and miRNA profiling,

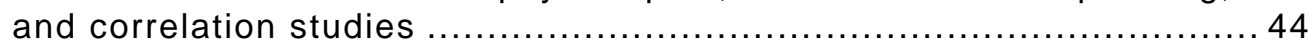

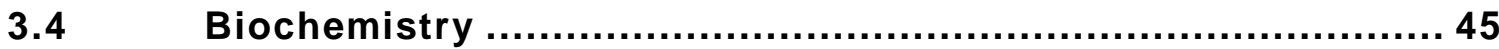

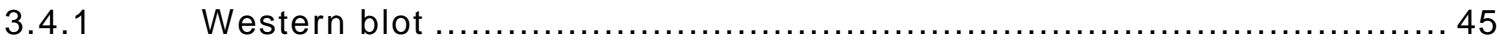

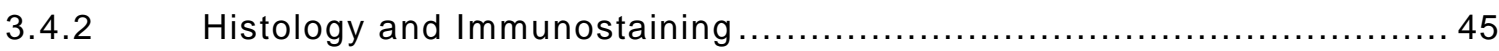

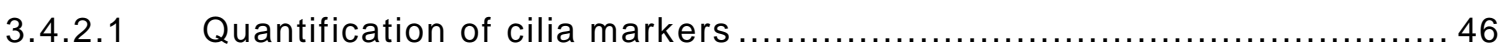

3.5 Molecular Biology ......................................................... 46

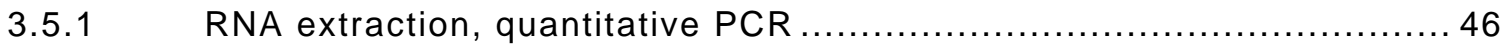

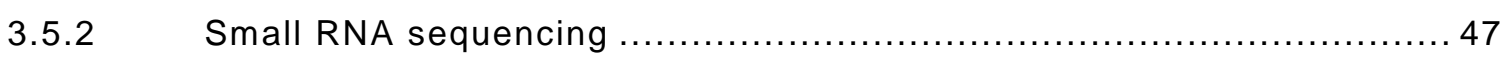

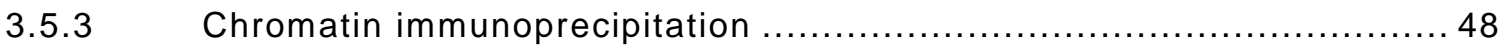

3.5.4 In situ hybridization, RNAscope .................................... 49

3.6 Microscopy, Video Microscopy ……................................ 50

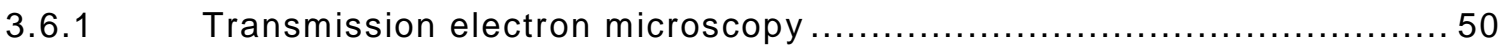

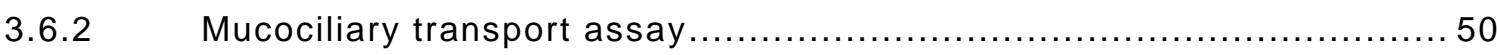

3.6.3 Video microscopy of spermatozoa and smooth muscle contraction of

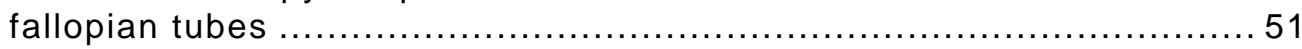

3.6.4 Imaging of cilia-generated bead-flow and cilia beating in the brain

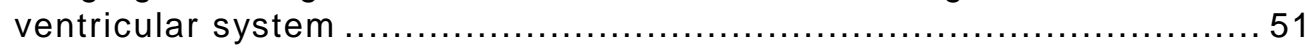

$3.7 \quad$ Statistical Analysis ................................................ 51 
4. RESULTS.

4.1 Chapter 1: Transcription factor TAp73 and microRNA-449 cooperate in multiciliogenesis

4.1.1 TAp73 is expressed in diverse multiciliated epithelia 54

4.1.2 TAp73 is crucial for the molecular circuit of multiciliogenesis in efferent ducts....

4.1.3 TAp73-driven transcriptional network regulates multiciliogenesis in fallopian tubes .......................................................... 58

4.1.4 Ciliary function in the brain is unaltered in the absence of $T A p 73 \ldots \ldots .60$

4.1.5 TAp73 regulates miR-34/449 family members in diverse multiciliated tissues

4.1.6 Combined deletion of TAp73 and miR449 impairs brain multiciliognesis

4.2 Chapter 2: miR449 protects airway cilia and healthy lung aging, thereby preventing COPD

4.2.1 Cilia-associated genes positively correlate with miR449 in COPD patients

4.2.2 miR449 is upregulated during airway epithelial differentiation and upon bronchial challenges ............................................... 86

4.2.3 miR449 deficiency reduces ciliation in Air-liquid interface cultures...... 89

4.2.4 miR449 is required for bronchial epithelial regeneration .....................90

4.2.5 miR449 $\%$ mice develop spontaneous COPD accompanied by an increased inflammatory response upon challenge .......................... 93

4.2.6 Aurora kinase A is a key player in miR449-regulated ciliary homeostasis and epithelial regeneration processes

4.2.7 miR449- mice accumulate ultrastructural cilia defects associated with reduced mucociliary clearance ....................................... 99

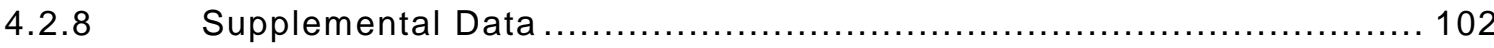

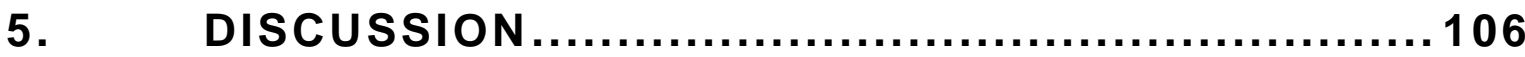

5.1 TAp73 - Tissue-dependent regulation of multiciliogenesis .. 106

5.1.1 Role of motile cilia in causing male infertility ........................... 107

5.1.2 Role of multiciliogenesis in ensuring female fertility .................... 110

5.1.3 Transcriptional regulation of multiciliogenesis in the ventricles of the brain - An exception

5.1.3.1 In ventricles, a robust network ensures multiciliogenesis in the absence of TAp73 ........ 113

5.1.3.2 Regulatory mechanisms explaining E2F4 and miR449 upregulation upon TAp73 loss . 115

5.1.3.3 Combined depletion of TAp73 and the miR449 cluster results in hydrocephalus 119

5.2 miR449 - Post-transcriptional regulation of airway multiciliogenesis

5.2.1 miR449 KO mice represent a novel model to study the role of motile cilia in COPD .............................................................. 120

5.2.2 Role of motile cilia in chronic respiratory diseases ..................... 121

5.2.3 Motile cilia homeostasis is not regulated by well-known miR-34/449 targets in miR449 $\mathrm{KO}$ mice 
5.2.4 A new role of miR449 in protecting motile airway cilia by repressing AURKA-mediated ciliary disassembly

5.2.5 Does miR449 repress the ciliary disassembly pathway at several nodes?

5.2.6 Cilia stabilizing agents as a new treatment option for COPD ............ 131

5.3

Conclusion

6. 


\section{LIST OF FIGURES}

Figure 1: Structure of motile and primary cilia. 3

Figure 2: Cellular composition of the murine airway epithelium and its regeneration potential.

Figure 3: Schematic representation of the male reproductive system. .... 10

Figure 4: Scheme of the female reproductive system $\ldots \ldots \ldots \ldots \ldots \ldots \ldots \ldots \ldots \ldots \ldots \ldots \ldots$

Figure 5: Sagittal view of the ventricular system of the murine brain..... 13

Figure 6: Model illustrating primary cilia disassembly. ................... 15

Figure 7: Transcriptional and post-transcriptional regulation of multiciliogenesis.

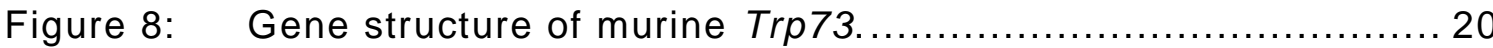

Figure 9: $\quad$ Seed sequence and genomic location of miR-34/449 family

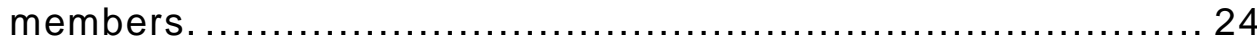

Figure 10: Naphthalene-induced airway epithelial injury. .................. 29

Figure 11: Schematic illustration of ALI cultures. ........................... 30

Figure 12: TAp73 deficiency impairs pulmonary function due to a reduced airway ciliation and mucociliary clearance.

Figure 13: TAp73 is a central regulator of airway multiciliogenesis......... 35

Figure 14: TAp73 is expressed in diverse multiciliated epithelial cells. ....5 54

Figure 15: Expression levels of TAp73 vary within different multiciliated tissues........................................................ 55

Figure 16: TAp73 deficiency results in loss of motile cilia in the efferent ducts.

Figure 17: TAp73 controls multiciliogenesis in the male reproductive tract.

Figure 18: Loss of TAp73 results in reduced ciliation in the oviducts. ..... 59

Figure 19: TAp73 controls multiciliogenesis in the oviducts. ...............60

Figure 20: TAp73 is expressed during brain development................. 61

Figure 21: Ablation of TAp73 does not results in loss of cilia in the ventricles of the brain.

Figure 22: TAp73 is dispensable for brain multiciliogenesis. 62

Figure 23: TAp73 functions through miR-34/449 family in brain multiciliogenesis.

Figure 24: E2F4 levels are upregulated in the ventricles of the brain in TAp73 KO mice................................................. 65

Figure 25: TAp73 functions through miR-34/449 family in brain multiciliogenesis.

Figure 26: miR449 cluster fails to exacerbate airway multiciliogenesis defects in the absence of TAp73.

Figure 27: Schematic diagram of the molecular mechanisms of TAp73driven multiciliogenesis in diverse tissues.

Figure 28: miR449 expression is correlated with the ciliation status in 
COPD patients.

Figure 29: miR449 is induced upon mucociliary differentiation in vitro. ... 87

Figure 30: miR449 is elevated in the lungs upon regenerative challenges....

Figure 31: miR34 expression is not induced in lungs from miR449-1mice.

Figure 32: miR449 depletion reduces ciliation in airway cell cultures. 90

Figure 33: Multiciliated airway regeneration is impaired in $\operatorname{miR} 449^{-1}$ mice.

Figure 34: Exposure to NTHi results in a loss of airway cilia in miR449 mice.

Figure 35: Motile airway cilia are strongly reduced in aged miR449-1 mice.

Figure 36: miR449 deficiency impairs pulmonary function to a similar extent as overserved in CS-exposed WT mice.

Figure 37: $\operatorname{miR} 449^{-/-}$mice develop spontaneous emphysema with increased inflammatory response.

Figure 38: miR449 depletion increases primary cilia disassembly. 96

Figure 39: miR449 represses ciliary disassembly by targeting Aurora kinase $\mathrm{A}$

Figure 40: Loss of miR449 generates cilia ultrastructural defects accompanied by reduced mucociliary clearance. 100

Figure 41: Proposed model of the protective role of miR449 in airway epithelial regeneration.

Figure 42: Proposed mechanism leading to male infertility in TAp73 KO mice. .......................................................... 109

Figure 43: Proposed mechanism leading to female infertility in TAp73 KO mice.

Figure 44: Scheme illustrating the robust network of brain multiciliogenesis.

Figure 45: Schematic diagram displaying possible mechanisms involved in the upregulation of E2F4 in TAp73 KO brains.... 116

Figure 46: Alterations in the expression of E2F4 and miR34b,c contribute to miR449 upregulation in TAp73 KO brains

Figure 47: Working model for the role of miR449 in the maintenance of motile airway cilia in response to environmental challenges. . 128

Figure 48: Hypothetical model of mechanisms contributing to the COPD phenotype in miR449 $\mathrm{KO}$ mice. 


\section{LIST OF SUPPLEMENTAL FIGURES}

Supplemental Figure 1: Expression of TAp73 in multiciliated tissues....... 70

Supplemental Figure 2: Loss of TAp73 impairs multiciliogenesis in the male reproductive duct.

Supplemental Figure 3: Binding of TAp73 is enriched at genomic loci from ciliary genes.

Supplemental Figure 4: TAp73 KO mice show defective motile cilia in fallopian tubes.

Supplemental Figure 5: TAp73 loss does not affect multiciliogenesis in the brain.

Supplemental Figure 6: Epithelial differentiation of CP cells is not affected upon TAp73 loss.

Supplemental Figure 7: Expression analysis of pro-ciliogenic factors in multiciliated tissues of TAp73 KO mice.

Supplemental Figure 8: $\mathrm{pRb} / \mathrm{E} 2 \mathrm{~F}$ pathway activity is not deregulated in TAp73 KO ventricles.

Supplemental Figure 9: TAp73xmiR449 KO mice possess reduced numbers of CP cilia, but not of ependymal cilia. .. 79

Supplemental Figure 10: Combined loss of TAp73 and miR449 does not affect epithelial differentiation of CP cells.

Supplemental Figure 11: Notch signaling is unaltered in developing ventricles of miR449 $\mathrm{KO}$ and TAp73xmiR449 KO mice.

Supplemental Figure 12: Additional loss of miR449 does not exacerbate ciliary defect in the absence of TAp73 in the airways and efferent ducts.

Supplemental Figure 13: Binding of TAp73 is enriched at the genomic locus from MLCK.

Supplemental Figure 14: $\operatorname{miR449^{-1}}$ ALI cultures express less cilia markers than their WT counterparts. 103

Supplemental Figure 15: Naphthalene treatment affects lung histology in WT and $\operatorname{miR} 449^{-/}$mice to a similar extent......... 104

Supplemental Figure 16: Ciliation appears normal in young miR449 mice.

Supplemental Figure 17: Young $m i R 449^{-/-}$mice do not display any signs of spontaneous COPD.

Supplemental Figure 18: Predicted targets involved in ciliary disassembly of miR449. 


\section{LIST OF TABLES}

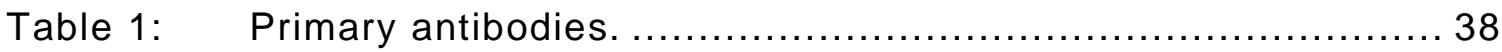

Table 2: $\quad$ Secondary antibodies. ........................................ 39

Table 3: $\quad$ Luciferase constructs. ............................................ 41

Table 4: $\quad$ Sequence information for primers used in RT-qPCR ............48

Table 5: $\quad$ Sequence information for primers used in ChIP-qPCR ..........49 


\section{LIST OF SUPPLEMENTAL TABLES}

Supplemental Table 1: Characteristics of COPD patients enrolled in the GLUCOLD study................................. 102

Supplemental Table 2: Top 10 enriched biological processes among miR34a-5p-positivly correlated genes in COPD patients.

Supplemental Table 3: Top 10 enriched biological processes among miR34b-5p-positivly correlated genes in COPD patients.

Supplemental Table 4: Top 10 enriched biological processes for miR34c-5p-positivly correlated genes in COPD patients. 


\section{ABBREVIATIONS}

\begin{tabular}{|c|c|}
\hline AA & Ambient air \\
\hline Ac- $\alpha-T U B$ & Acetylated-alpha-tubulin \\
\hline ALI & $\underline{\text { Air-liquid interface }}$ \\
\hline AQP1 & Aquaporin 1 \\
\hline ARL13B & $\underline{A D P}$-ribosylation factor-like $\underline{13 b}$ \\
\hline AURKA & 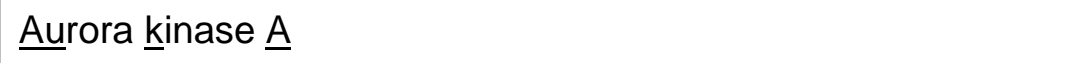 \\
\hline BAL & Bronchoalveolar lavage \\
\hline BALF & Bronchoalveolar lavage fluid \\
\hline BB & Basal body \\
\hline BSA & Bovine serum albumin \\
\hline Btg4 & Btg Anti-Proliferation factor $\underline{4}$ gene \\
\hline CaM & $\underline{\text { Calmodulin }}$ \\
\hline CC10 & $\underline{\text { Club cell }} \underline{10} \mathrm{kDa}$ protein \\
\hline CCNO & 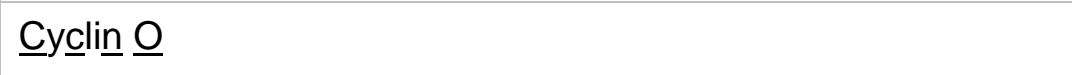 \\
\hline Cf & $\underline{\text { Confer }}$ \\
\hline CHAPS & 3-[(3-cholamidopropyl) dimethylammonio]-1-propanesulfonate \\
\hline ChIP & $\underline{\text { Chromatin immunoprecipitation }}$ \\
\hline COPD & 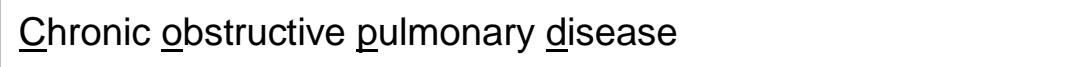 \\
\hline CP & $\underline{\text { Choroid plexus }}$ \\
\hline CS & $\underline{\text { Cigarette smoke }}$ \\
\hline Ctrl & Control \\
\hline CYP & Cytochrome $\underline{\mathrm{P}} 450$ \\
\hline D & Day \\
\hline DAPI & 4, 6-Diamidin-2-phenylindol \\
\hline DDX6 & $\underline{D E A} \underline{\mathrm{D}}$-box $\underline{x}$ helicase $\underline{6}$ \\
\hline DE & Differential expression \\
\hline DEP & Diesel exhaust particle \\
\hline DMEM & Dulbecco`s Modified EEagle Medium \\
\hline DNAH5 & Dynein axonemal heavy chain $\underline{5}$ \\
\hline DNAl1 & Dynein axonemal intermediate chain 1 \\
\hline
\end{tabular}




\begin{tabular}{|c|c|}
\hline DNALI1 & Dynein axonemal light intermediate chain $\underline{1}$ \\
\hline $\mathbf{E}$ & Embryonic day \\
\hline ED & Efferent duct \\
\hline Epi & Epididymis \\
\hline FCS & Fetal calf serum \\
\hline FDR & False discovery rate \\
\hline FEV 1 & forced expiratory volume in 1 second \\
\hline FOXJ1 & Forkhead box $\underline{\mathrm{J} 1}$ \\
\hline Fr & Frozen cryosections \\
\hline FT & Fallopian tube \\
\hline GEMC1 & 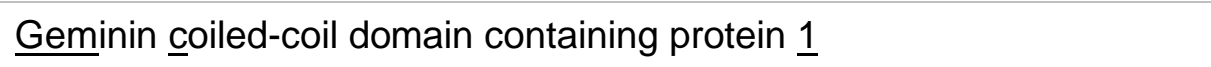 \\
\hline GEO & Gene Exxpression Omnibus \\
\hline GLUCOLD & 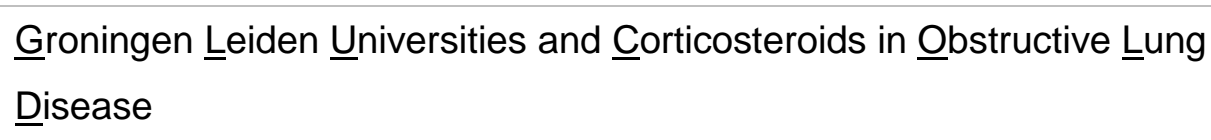 \\
\hline GSEA & Gene-set enrichment analysis \\
\hline GTP & Guanosine triphosphate \\
\hline H & Hour(s) \\
\hline H\&E & Hematoxylin and eosin \\
\hline HDAC6 & 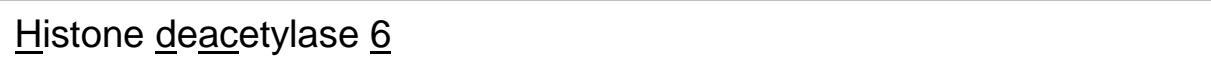 \\
\hline HEF1 & Human enhancer of filamentation 1 \\
\hline HSC70 & Heat shock cognate $\underline{70} \mathrm{kDa}$ protein \\
\hline I.p. & Intraperitoneal \\
\hline ICC & Immunocytochemistry \\
\hline IDA & Inner dynein arm \\
\hline IF & Immunofluorescence \\
\hline IFT & Intraflagellar transport \\
\hline IHC & Immunohisistochemistry \\
\hline IL & Interleukin \\
\hline ISH & In situ hybridization \\
\hline IVC & Inspiratory vital capacity \\
\hline KO / -/- & Knockout \\
\hline Kv10.1 & potassium voltage-gated channel subfamily $\mathrm{H}$ member 1 (KCNH1) \\
\hline
\end{tabular}




\begin{tabular}{|c|c|}
\hline MCC & Multiciliated cell \\
\hline MCIDAS & $\begin{array}{l}\text { Multiciliate differentiation and DNA synthesis-associated cell cycle } \\
\text { protein }\end{array}$ \\
\hline MEF & Mouse embryonic fibroblast \\
\hline Min & Minutes \\
\hline miRNA, miR & $\underline{\text { MicroRNA }}$ \\
\hline MLCK & Myosin light chain kinase \\
\hline MMP & Matrix metalloproteinases \\
\hline MTEC & Mouse tracheal epithelial cell \\
\hline MYB & V-myb avian myeloblastosis viral oncogene homolog \\
\hline N.s. & Non-significant \\
\hline NE & Neutrophil elastase \\
\hline NTHi & Nontypeable $\underline{\text { Haemophilus influenzae }}$ \\
\hline ODA & 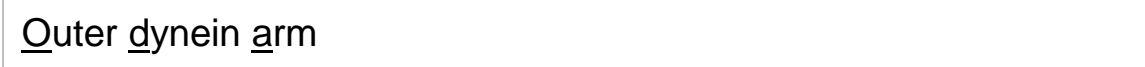 \\
\hline OTX2 & Orthodenticle homeobox $\underline{2}$ \\
\hline $\mathbf{P}$ & Paraffin embedded sections \\
\hline PCD & Primary ciliary $\underline{\text { dyskinesia }}$ \\
\hline PFA & Paraformaldehyde \\
\hline PBS-T & Phosphate-buffered saline containing $0.1 \%$ Triton X-100 \\
\hline PIFO & Pitchfork \\
\hline Pre-miRNA & $\underline{\text { Precursor miRNA }}$ \\
\hline $\mathbf{R b}$ & $\underline{\text { Retinoblastoma protein }}$ \\
\hline RFX2/3 & 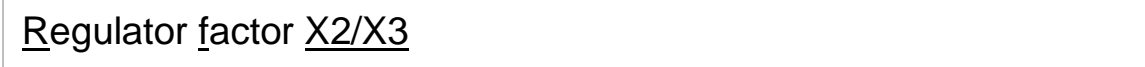 \\
\hline RNA & $\underline{\text { Ribonucleic acid }}$ \\
\hline RNA-seq & $\underline{\text { RNA-sequencing }}$ \\
\hline RT & Room temperature \\
\hline RT-qPCR & Reverse transcription quantitative polymerase chain reaction \\
\hline SAM & 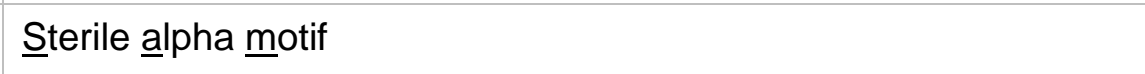 \\
\hline SEM & Standard error of the mean \\
\hline SNP & Single nucleotide polymorphisms \\
\hline snRNA & 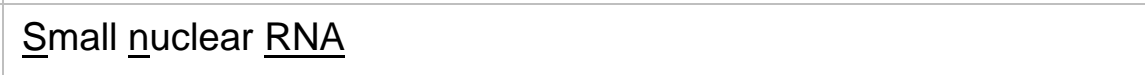 \\
\hline SSC & Saline-sodium citrate buffer \\
\hline
\end{tabular}




\begin{tabular}{|c|c|}
\hline TA & Iransactivation domain \\
\hline TEM & Transmission electron microscopy \\
\hline TIMP1 & Tissue inhibitor of metalloproteinases 1 \\
\hline TNF & Tumor necrosis factor \\
\hline TNRC6 & Trinucleotide repeat containing $\underline{6}$ \\
\hline Trp73 & Transformation-related protein $\underline{73}$ \\
\hline TTR & Transthyretin \\
\hline UTR & Untranslated region \\
\hline W & Week \\
\hline WB & 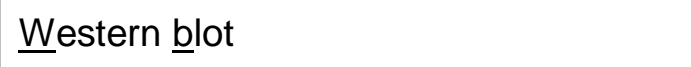 \\
\hline WT & Wild type \\
\hline Y-TUB & Gamma-tubulin \\
\hline 4E-T & elF $\underline{4 E}$-Transporter \\
\hline
\end{tabular}




\section{ABSTRACT}

Multiple motile cilia are present on specialized epithelial cells lining the airways, reproductive ducts, and brain ventricles. Cilia beating contributes to fluid movement and particle transport along the multiciliated surfaces. Dysfunction of motile cilia leads to ciliopathies such as primary ciliary dyskinesia, which is mainly characterized by respiratory infections, infertility, and - in rare cases - hydrocephalus. Due to the diverse vital functions of motile cilia, multiciliogenesis must be tightly controlled at both the transcriptional and post-transcriptional level. We recently identified the tumor suppressor and transcription factor TAp73 as a central regulator of airway multiciliogenesis by activating the transcription of several different pro-ciliogenic factors.

Here, we show that TAp73 elicits its pro-ciliogenic function not only in the airways but also in other multiciliated tissues, albeit to different extents. Depletion of TAp73 results in a profound loss of motile cilia accompanied by diminished expression of several pro-ciliogenic factors including Foxj1, Rfx2, Rfx3, and axonemal dyneins Dnai1 and Dnali1 in the murine male and female reproductive tracts. As male and female TAp73 KO mice are infertile, we propose that defective multiciliogenesis in these mice contributes to the infertility phenotype by preventing proper gamete and zygote transport. Interestingly, multiciliated cells of the brain ventricles of TAp73 KO mice maintain their multiciliogenesis program by upregulating two well-established drivers of multiciliogenesis, namely E2F4 and miR449. Consistently, mice depleted for both TAp73 and miR449 display defective brain multiciliogenesis as these mice possess less and shorter motile cilia in the choroid plexus, which might contribute to the observed hydrocephalus in these mice. In summary, our data show that the role of TAp73 in multiciliogenesis is tissue-dependent and the interplay between transcriptional and post-transcriptional regulators ensures the development of functional motile cilia.

As we have demonstrated that miR449 is involved in brain multiciliogenesis, we next aimed to evaluate the role of miR449 in another multiciliated tissue, namely the airways. Coordinated beating of airway cilia contributes to mucociliary clearance, thereby facilitating airway defense and ensuring proper respiratory function. Moreover, multiciliated cells also participate in bronchial epithelial regeneration, a process which is impaired in patients with chronic obstructive pulmonary disease (COPD) in addition to a defective mucociliary clearance. Here, for the first time, we report a link between cilia-related genes and miR449 in COPD patients using genome-wide transcriptome analysis. Since miR449 is strongly upregulated during mucociliary differentiation and bronchial epithelial regeneration, miR449 depletion in mice results in an impaired ciliated epithelial regeneration upon exposure to various damaging challenges. This shows that miR449 is an important contributor to mucociliary regeneration and it does so by targeting Aurora kinase A (AURKA), a key player in the ciliary disassembly pathway. Consequently, deficiency of miR449 in mice increases AURKA levels, reduces airway cilia upon challenge and mucociliary clearance, which 
thereby triggers spontaneous emphysematous manifestations of COPD. Thus, our study provides a connection between miR449, defective cilia maintenance, and COPD development.

Altogether, our study shows that miRNAs and tumor suppressors form a gene regulatory network to protect multiciliogenesis in different organs, thereby preventing the onset of diseases. 


\section{INTRODUCTION}

\subsection{Cilia}

\subsubsection{Cilia classification and structure}

Cilia are highly conserved, microtubule-based surface organelles with important mechanical and sensory functions. In vertebrates, cilia are present in diverse cell types and tissues with varying length, motility, and number per cell. A common structural property observed in all types of cilia includes a ring-shaped core consisting of alpha ( $\alpha$ )- and beta ( $\beta$ )-microtubule dimers arranged in a nine-fold symmetry that defines the ciliary axoneme (Figure 1a and $b$ ) (Satir and Christensen, 2007b). Several post-translational modifications, including acetylation of $\alpha$-tubules, occur on the axoneme, and influence the stability of the cilia (Wloga et al., 2017). The axoneme is nucleated by a specialized centriole composed of microtubule triplets called basal body (BB) (Figure 1a).

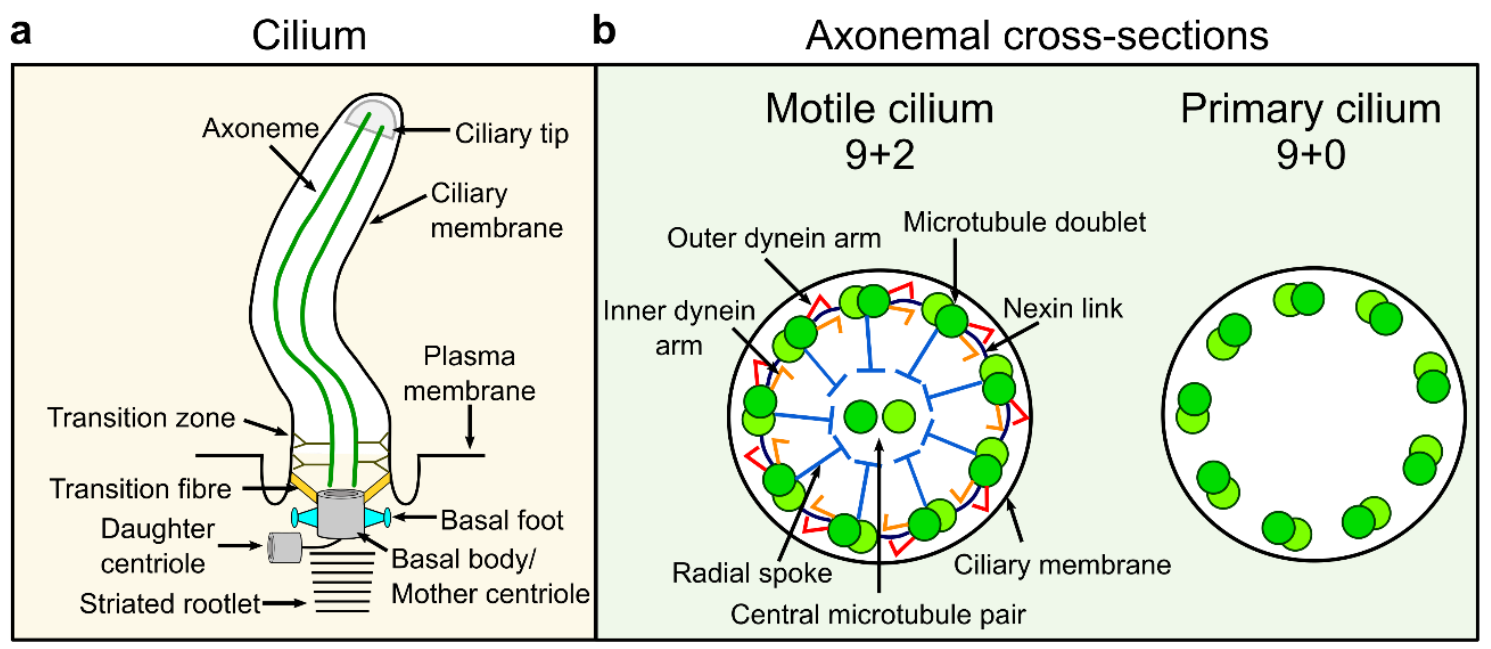

Figure 1: Structure of motile and primary cilia.

a) Schematic representation of the core structures of motile and primary cilia. The BB with its appendages nucleates the ciliary axoneme that consists of microtubules. b) Axonemal cross-sections from a motile (left) and primary (right) cilium. A motile cilium consists of a 9+2 microtubule structure to which several motilityrelated protein complexes are attached. A primary cilium lacks the accessory structures necessary for cilia motility as well as the central pair and thus, comprises a 9+0 microtubule arrangement. Adapted from (Ishikawa and Marshall, 2011).

The ciliary BB displays several appendages as mentioned below.

1) Ciliary rootlets - connect the BB to the nucleus of the cell and provide structural support for the cilium.

2) Transition fibers - anchor the BB to the membrane and act as a docking platform for ciliary proteins prior to their import into the ciliary compartment (Kobayashi and Dynlacht, 2011). 
3) Basal feet - are associated with cytoplasmic microtubules and are necessary for the polarized alignment of BBs required for motile cilia beating (Kunimoto et al., 2012; Garcia and Reiter, 2016).

Additional ciliary sub compartments are the ciliary tip, the ciliary plasma membrane, and the transition zone (Figure 1a). The latter is characterized by Y-shaped linkers connecting the outer axonemal microtubule doublets to the ciliary membrane, thereby forming a gate that controls ciliary protein passage into the cilium (Kobayashi and Dynlacht, 2011).

Cilia can be classified into primary and motile cilia depending on their ability to move. Single, immotile primary cilia are generated by almost all mammalian cell types, whereas only some specialized epithelial cells lining the airways, reproductive ducts, and brain ventricles form multiple motile cilia per cell (Satir and Christensen, 2007b). Motile cilia and their function in different organs are described in more detail in the following sections, as they represent the main topic of this thesis.

\subsubsection{Motile cilium}

Depending on the organ, multiciliated cells (MCCs) assemble up to 300 motile cilia, which beat rhythmically to facilitate fluid, gamete, and zygote transport (Satir and Christensen, 2007b). Planar cell polarity orients the BBs of motile cilia in each MCC and within the tissue, thereby allowing a rhythmic unidirectional beating. Parallel alignment of multiple BBs within each MCC and across the tissue are defined as rotational planar polarity and tissue-level planar polarity, respectively. In MCCs of the airways and reproductive ducts, BBs cover the entire apical cell surface. However, in the ventricles of the brain, cilia are clustered at one side of the apical area, a feature termed translational polarity (Wallingford, 2010).

Motile cilia consist of two central single microtubules called the central pair, which is surrounded by an outer ring composed of nine peripheral microtubule doublets (termed 9+2 structure) (Figure 1b). The 9+2 arrangement of microtubules also defines the core of a flagellum, which is present for example on sperm cells. In most cases, flagellated cells possess a single motile monocilium required for their movement (Satir and Christensen, 2007b). Several accessory structures are attached to the outer microtubule doublets including nexin links, radial spokes, nexin-dynein-regulator complexes, and inner and outer dynein arms (IDAs and ODAs respectively). These structures operate in unison to ensure cilia motility. Axonemal dynein arms anchored to the a-tubule are large ATP-dependent multiprotein complexes that move along the length of the $\beta$-tubule towards the minus end of the axoneme (anchored in the BB), which results in microtubule sliding. Due to the unidirectional movement of the dyneins, their activity is switched on only on one side of the axoneme to induce microtubule sliding in one direction, while the dynein arms of the other half are inactive and only become active during the recovery stroke into the opposite direction. Thus, the activity of the dynein motors must be tightly regulated to generate an 
efficient ciliary beat. This regulation is achieved by other microtubule-associated structures, namely the radial spokes and nexin-dynein-regulator complexes, which receive signals from the central pair apparatus and subsequently transmit these to both dynein arms (Satir et al., 2014). Thus, loss of function of one or more proteins of this complex network disrupts the motility of cilia and triggers the onset of diseases in many different organ systems as described in section 1.4 (Fliegauf et al., 2007).

\subsubsection{Primary cilium}

Primary cilia are composed of a 9+0 microtubule structural arrangement in comparison to the 9+2 arrangement observed in motile cilia. Primary cilia lack the central pair and other key protein complexes that are necessary for cilia motility (Figure 1b). Besides their sensory function in detecting mechanical and chemical stimuli, primary cilia coordinate multiple signaling pathways that play an important role during development and tissue homeostasis. Typically, primary cilia are solitary. However, clusters of multiple immotile primary cilia are found on some sensory cells such as the olfactory cells (Satir and Christensen, 2007b).

\subsection{Location and function of motile cilia in diverse tissues}

Motile cilia are found in the respiratory tract, male and female reproductive system, as well as in the ventricles of the brain. Location and function of MCCs in these diverse tissues are discussed below.

\subsubsection{Respiratory tract}

The respiratory system is divided into the upper and lower respiratory tract. The nasal cavity, pharynx, and larynx are part of the upper airway tract, whereas the trachea, bronchial tree, and lungs belong to the lower airways. The trachea divides into two primary bronchi, each leading into a lung, where they branch into smaller bronchi. As bronchi become narrower, they are considered as bronchioles, which terminate into alveoli that are responsible for gas exchange. The respiratory tract is lined with an epithelium that changes its cellular composition along the proximal to distal axis (Figure 2a). 


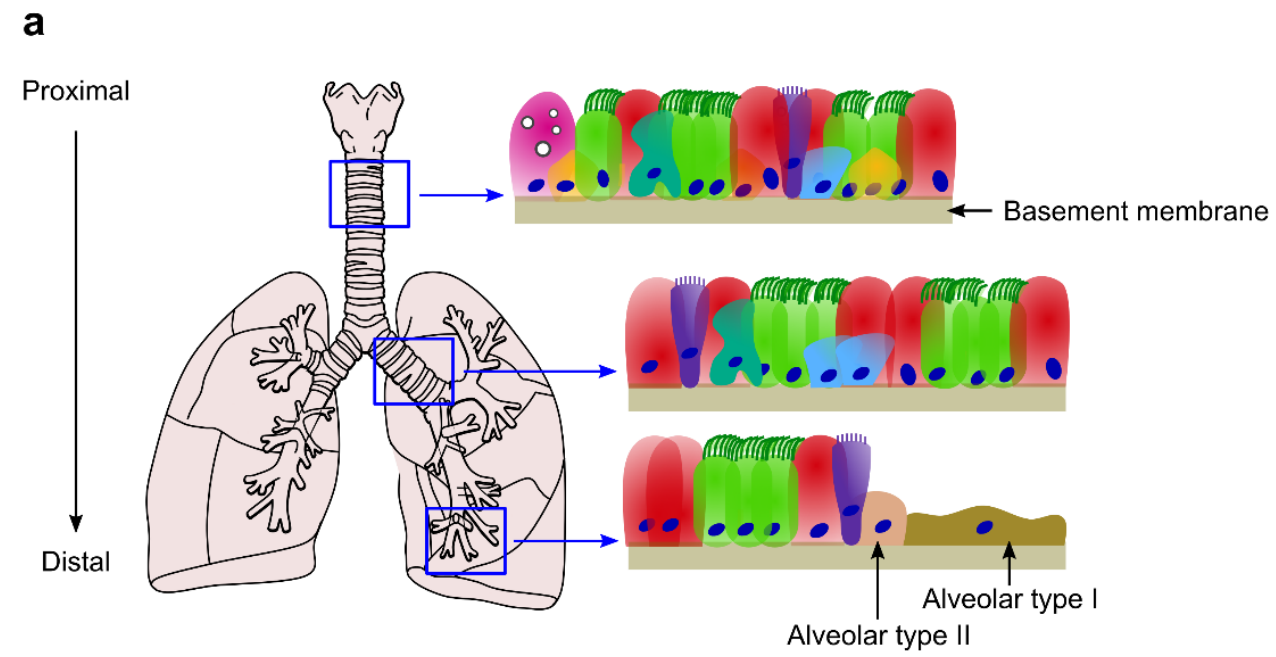

b

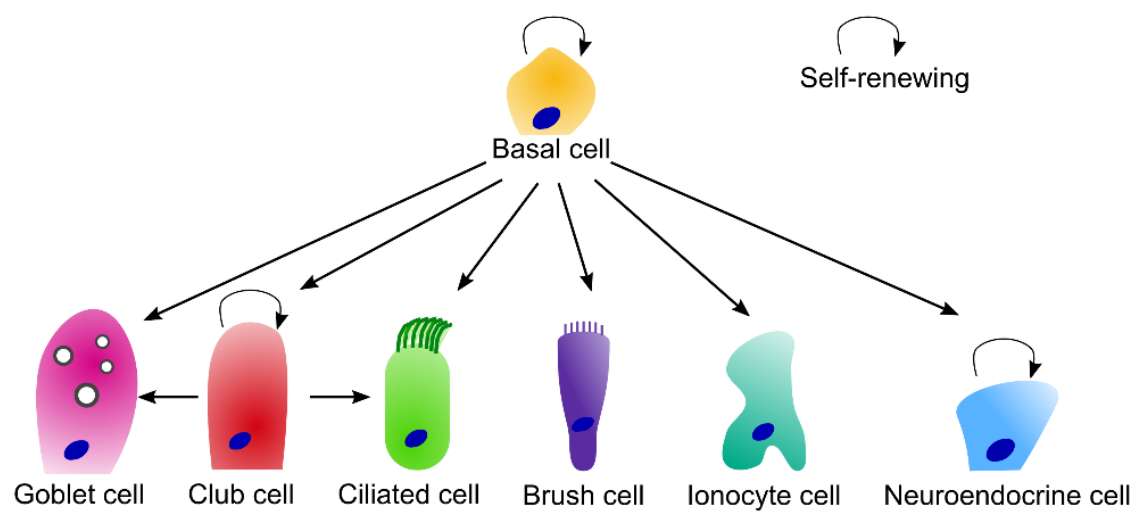

Figure 2: Cellular composition of the murine airway epithelium.

a) The cellular composition of the airway epithelium differs along the proximal-distal axis. The epithelium of the conducting airways mainly consists of basal, club, and ciliated cells. Rare cell types are goblet, brush, ionocyte, and neuroendocrine cells. Notice that further down the bronchial tree, basal cells are absent in the murine lung. b) Airway epithelial cells can regenerate the respiratory epithelium under homeostatic conditions. Basal cells have the capacity of self-renewal and differentiate into all different kinds of airway epithelial cells. Hence, basal cells are the stem cells of the large airways. Progenitor cells of the small airways are club cells, which can transdifferentiate into ciliated and goblet cells. Adapted from (Rackley and Stripp, 2012; Schilders et al., 2016; Montoro et al., 2018).

The trachea and bronchi are covered by a pseudostratified columnar epithelium composed of a variety of cell types, which mainly include basal, ciliated, and non-ciliated secretory cells (club cells). In contrast, smaller airways are predominantly comprised of ciliated and club cells that form the columnar epithelium (Rackley and Stripp, 2012). Moreover the conducting airways are composed of goblet, brush, ionocyte, hillock (not shown in Figure 2), and neuroendocrine cells, which are quite rare cell type populations (Montoro et al., 2018). Additionally, the alveolar epithelium contains alveolar type I and II cells (pneumocytes).

In general, these various cell types are found in human and mouse airways; however, the distribution differs slightly between these two species. Basal cells are restricted to the 
tracheal region in mice, whereas in humans, they are found throughout the epithelium with their amount declining with airway size (Rackley and Stripp, 2012).

Arrangement of the individual lung lobes is another pivotal difference between the human and mouse respiratory tract. The human lung is segmented into three lobes on the right und two lobes on the left, while the murine lung consists of four right lobes and a single left lobe (Fox et al., 2006).

All cells of the respiratory epithelium function together to protect against the entry of foreign pathogens or particles by not only acting as a physical barrier and mucociliary escalator but also by contributing to the innate immune response. The innate immune response of the respiratory epithelium is achieved by the secretion of soluble factors such as cytokines and chemokines by airway epithelial cells. These inflammatory mediators recruit immune cells and thereby activating the innate and adaptive immunity. Thus, the airway epithelium contributes directly to the host defense. Due to the constant exposure of airways to external stimuli, it is important that the airway epithelium is renewed upon injury to restore its functions (Tam et al., 2011).

\subsubsection{Respiratory epithelial cells and their function}

The various respiratory epithelial cells including basal, club, multiciliated, goblet, neuroendocrine, brush, ionocyte, and hillock cells are vital for maintaining airway homeostasis and regeneration (Rackley and Stripp, 2012; Montoro et al., 2018; Plasschaert et al., 2018).

\section{Basal cells}

Basal cells are a population of undifferentiated progenitor cells that cover the basement membrane without being exposed to the airway lumen. These progenitor cells are considered to be the stem cells of the airways due to their ability to differentiate into all the distinct cell types that form the respiratory epithelium (Figure 2b). Hence, basal cells contribute to the homeostasis and repair of the large airways of the epithelium due to their predominant expression there. In contrast, in the lower respiratory tract, club cells are the main progenitor cells (Rackley and Stripp, 2012).

\section{Club cells}

Club cells are predominantly found in the small airways. They can proliferate and differentiate into ciliated and goblet cells (Figure 2b). Apart from their role as progenitor cells, club cells secrete proteins such as the club cell $\underline{10} \mathrm{kDa}$ protein (CC10) (Hiemstra and Bourdin, 2014) and surfactant proteins (SP-A, SP-B, and SP-D) into the fluid lining the respiratory bronchioles (Han and Mallampalli, 2015). Surfactant proteins are essential for lowering the surface tension at the alveolar and bronchiolar air-liquid interface (ALI) (SP-B, 
SP-C), but also contribute to the host defense (SP-A, SP-D) (Han and Mallampalli, 2015). Furthermore, club cells are the only cell type of the respiratory epithelium that express P450 monooxygenases necessary for the detoxification of substances, including the polycystic aromatic hydrocarbon naphthalene (described in section 1.5.1.1) (Tam et al., 2011).

\section{Multiciliated cells}

MCCs are situated in the epithelium of the small and large airways and arise from club cells and basal cells respectively (Tam et al., 2011; Montoro et al., 2018). Under homeostatic condition, MCCs are differentiated columnar cells. However, ciliated cells can transdifferentiate into goblet cells during inflammatory processes as ciliated/goblet cell transdifferentiation is mediated by inflammatory cytokines (Tyner et al., 2006; Gomperts et al., 2007; Turner et al., 2011).

The coordinated beating of multiple motile cilia on MCCs contributes to the mucociliary clearance by transporting pathogens or particles trapped in the mucus out of the airways (Tam et al., 2011). Consequently, defective motile cilia trigger the onset of airway diseases

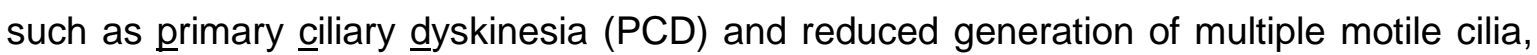
which is another mucociliary clearance disorder (Boon et al., 2014; Wallmeier et al., 2014). Moreover, dysfunctions of motile cilia are also involved in the pathogenesis of acquired

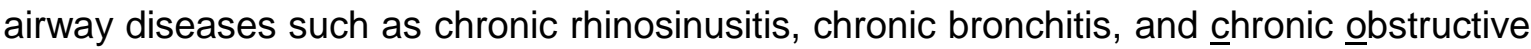
pulmonary disease (COPD) (Tilley et al., 2015) (described in section 1.4).

Efficient mucociliary clearance depends not only on the movement of motile cilia but also on the amount and viscoelasticity of the periciliary layer and the overlying mucus. The periciliary layer surrounds motile cilia, supports coordinated cilia movement, and serves as a barrier between the mucus trapped particles and the cell surface. Impairment of the periciliary layer (e.g. by dehydration) results in the collapse of the mucus layer, which in turn leads to defective mucociliary clearance as observed in cystic fibrosis (described in section 1.2.1.1). The mucus layer is mainly composed of glycoproteins, which are secreted by goblet cells and submucosal glands (Bustamante-Marin and Ostrowski, 2017).

\section{Goblet cells}

Goblet cells are present only in small numbers in the conducting airways and help in regulating mucus production. In response to inflammatory signals (e.g. cytokines) the number of goblet cells increase (metaplasia, hyperplasia), which results in hypersecretion of mucus that in turn triggers airway obstruction associated with chronic airway diseases such as chronic bronchitis (Rackley and Stripp, 2012). 


\section{Neuroendocrine cells}

Pulmonary neuroendocrine cells are present throughout the respiratory epithelium and are commonly found at innervated airway branch points, where they often form clusters, which are termed neuroepithelial bodies (Rackley and Stripp, 2012). Neuroendocrine cells possess neuro-immunomodulatory functions and are required for allergen-induced responses (Sui et al., 2018). Increased numbers of neuroendocrine cells have been found in diseases associated with chronic inflammation such as COPD (Gu et al., 2014). Moreover, the microenvironment of the neuroepithelial body functions in maintaining progenitor cells capable of epithelial regeneration after injury for example by naphthalene (described in section 1.5.1.1) (Reynolds et al., 2000).

\section{Brush cells}

Brush cells (also called tuft cells) are characterized by the presence of microvilli (Reid et al., 2005). They have been less intensively studied. Until now, it has been shown that brush cells line the entire airway and alveolar epithelium (Reid et al., 2005) and act as epithelial chemosensors by detecting irritants via the canonical taste transduction cascade (Krasteva et al., 2011).

\section{lonocyte and hillock cells}

Recently, two new cell types of the respiratory epithelium have been identified, named ionocytes and hillocks (Montoro et al., 2018; Plasschaert et al., 2018). lonocytes represent a rare population of pulmonary cells that function in fluid regulation at the airway surface due to their high expression of the chloride channel called cystic fibrosis transmembrane conductance regulator (CFTR) (Montoro et al., 2018; Plasschaert et al., 2018). Mutation in CFTR gene are associated with the pathophysiology of cystic fibrosis. Cystic fibrosis is a multiorgan disease, which is characterized amongst other things by chronic airway infections. Dysfunction of CFTR leads to several changes in the airways, including dehydration and acidification (Boucher, 2007; Shah et al., 2016). Dehydration of the airway surface fluid caused by an increased fluid absorption into the cell is accompanied by a series of consecutive events, including collapse of the periciliary fluid layer, increased mucus viscosity, and impaired mucociliary clearance (Boucher, 2007).

The second newly identified cell type is the hillock cell, which is characterized by a high cellular turnover that is linked to immunomodulation and barrier function (Montoro et al., 2018). 


\subsubsection{Male reproductive tract}

The male reproductive system consists of the penis, epididymides, and testes. The last two organs are connected by the efferent ducts (EDs) that are lined by MCCs (Ilio \& Hess, 1994) (Figure 3).

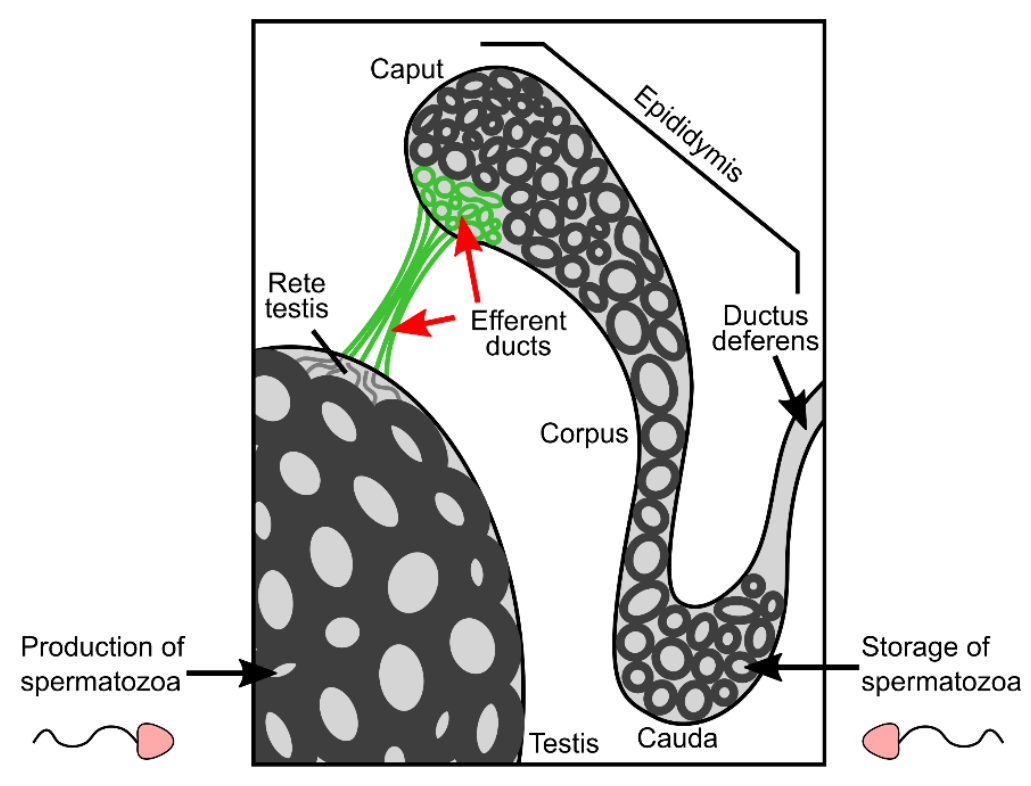

\section{Figure 3: Schematic representation of the male reproductive system.}

The male reproductive tract consists of the testis, ED (red arrow), epididymis, and penis (not shown). Spermatozoa produced in the testis are transported via the EDs to the epididymis where they further mature and are stored until ejaculation. EDs highlighted in green contain MCCs, which are involved in the transport of spermatozoa. Adapted from (Wildung et al., 2018).

The testis is made up of seminiferous tubules, which are lined by a germinal epithelium consisting of multiple layers of varying cell types, including Sertoli cells and cells of the spermatogenic cell lineage. The process by which functional spermatozoa are produced within the germinal epithelium is referred to as spermatogenesis. Spermatogenesis comprises several successive developmental stages including the formation of spermatogonial germ cells, spermatocytes, spermatids, and spermatozoa via mitotic and meiotic divisions. During the last developmental step, morphological changes occur resulting in the formation of a flagellum on the spermatozoa. The nutritional and structural support from the Sertoli cells help in the development of spermatozoa. Hence, these Sertoli cells are also known as nurse cells. Sertoli cells in turn are regulated by testosterone secreting Leydig cells, which are located in the interstitial tissue of the testis (Cooke and Saunders, 2002). A transcription factor, which is expressed in germ cells (Hamer et al., 2001; Holembowski et al., 2014; Inoue et al., 2014) and interferes with spermatogenesis, is TAp73. Briefly, TAp73 is required for spermatogonia maintenance, spermatid maturation, and germ cell adhesion to Sertoli cells (Holembowski et al., 2014; Inoue et al., 2014) (described in section 1.3.4.2.2). 
Following spermatogenesis, the resulting spermatozoa, which are not yet motile, are transported from the testis to the epididymis via the EDs (Tulsiani and Abou-Haila, 2012). EDs are composed of a pseudostratified columnar epithelium containing ciliated, nonciliated, and basal cells. The epithelium of the EDs is surrounded by layers of connective tissues and smooth muscles. The contraction of the smooth muscles is required for efficient transport of the spermatozoa along this conduit (Hess, 2002). Furthermore, EDs are involved in testicular fluid reabsorption, which is essential for spermatozoa concentration. Fluid reabsorption might be facilitated by the beating of multiple motile cilia resulting in the distribution of the luminal content. Thus, MCCs in the EDs are required for proper spermatogenesis and male fertility. Of note, motile cilia of the EDs possess a rotational beating pattern and thus, might be unable to push the spermatozoa towards the epididymis (Hess, 2015). In contrast, motile cilia of the airways, fallopian tubes (FTs), and brain ventricles are involved in the coordinated transport of substances towards one direction (Spassky and Meunier, 2017).

After passing the EDs, spermatozoa undergo further maturation as they travel through the epididymis yielding motile spermatozoa, which are stored in the cauda epididymis until ejaculation. Once in the female reproductive tract, these spermatozoa undergo capacitation, a process that creates a fully functional spermatozoa, which can fertilize an oocyte (Tulsiani and Abou-Haila, 2012).

\subsubsection{Female reproductive tract}

The female reproductive tract includes the vagina, uterus, FT, and the ovary (Figure 4). The ovary releases the oocyte into the abdominal cavity, where it is caught by the ciliated fingerlike fimbriae that extend from the FT. The FT is lined by a pseudostratified epithelium that is mainly composed of secretory and ciliated cells. The beating of multiple motile cilia along with smooth muscle contraction is responsible for the transport of the oocyte and the zygote (fertilized oocyte) along the FT towards the uterus where implantation occurs. Since impairment of smooth muscle contraction did not affect the velocity of oocyte movement, ciliary activity seems to be the main driving force for tubal transport. Hence, ciliary function is necessary for female fertility (Ezzati et al., 2014). 


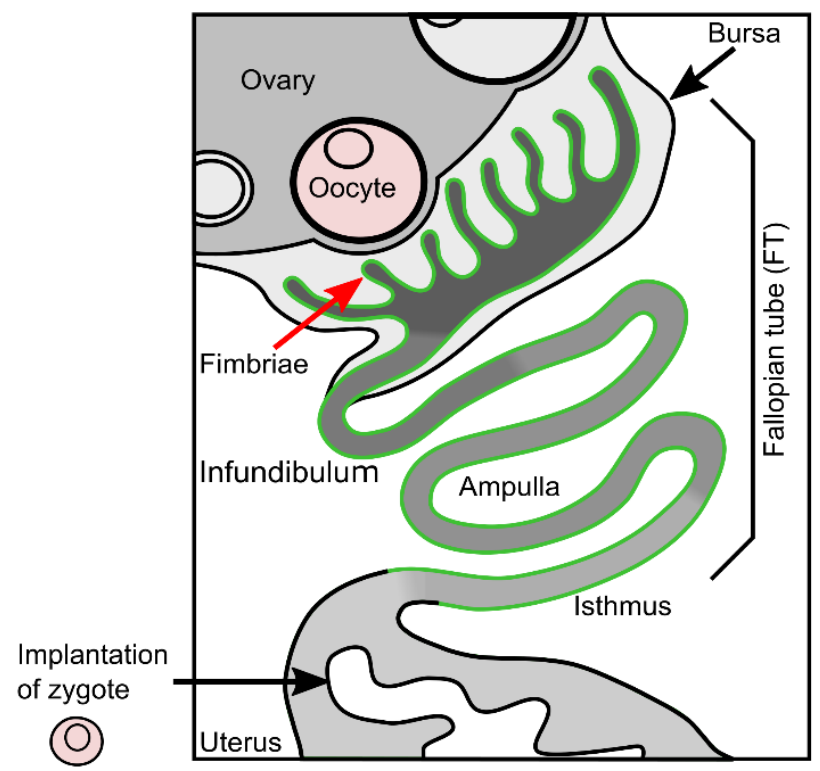

Figure 4: Scheme of the female reproductive system.

The oocyte released from the ovary is caught by the fimbriae (red arrow) and transported along the FT towards the uterus, where implantation of the zygote takes place. The transport along the FT is achieved by coordinated cilia beating. Regions containing MCCs are highlighted in green. Adapted from (Wildung et al., 2018).

\subsubsection{Ventricular system of the brain}

The ventricular system of the brain consists of four interconnected ventricles: two lateral ventricles merging into the third ventricle, which is connected to the fourth ventricle by the cerebral aqueduct (Figure 5). The fourth ventricle lies in the brain stem and continues to the central canal of the spinal cord. The ventricular system as well as the central canal are covered by a single-layered epithelium consisting of ependymal cells (Jiménez et al., 2014). Ependymal cells are derived from radial glial cells and postnatally develop multiple motile 9+2 cilia (Spassky et al., 2005). In contrast to the airways and reproductive ducts where MCCs are frequently regenerated, the brain MCCs are produced only once during embryonic development and their regenerative capacity after injury is quite low (Spassky et al., 2005; Kuo et al., 2006; Luo et al., 2015). Coordinated beating of ependymal cilia contributes to the circulation of the cerebrospinal fluid, which is important for the removal of harmful substances as well as distribution of nutrients within the ventricular system. The cerebrospinal fluid is secreted by epithelial cells of the choroid plexus (CP), which protrudes into the brain ventricles (Jiménez et al., 2014). The CP epithelial cells contain specialized cilia, which display motility around the perinatal period but then shifts to the non-motile $9+0$ cilia (Narita and Takeda, 2015).

Impairment of the cerebrospinal fluid flow, production, or absorption, but also obstructions in the ventricular system leads to the accumulation of cerebrospinal fluid, resulting in the development of hydrocephalus that is characterized by dilated ventricles. As efficient cilia beating is required for the circulation of the cerebrospinal fluid, defects in cilia motility can 
cause hydrocephalus. Thus, hydrocephalus is commonly observed in mouse models of PCD, whereas it is rare among humans with PCD (Lee, 2013). The brain morphology of mice with a longer and narrower aqueduct compared to humans, may explain the increased vulnerability for the development of hydrocephalus in mice with ciliary defects (IbañezTallon et al., 2002; Ibanez-Tallon, 2004).

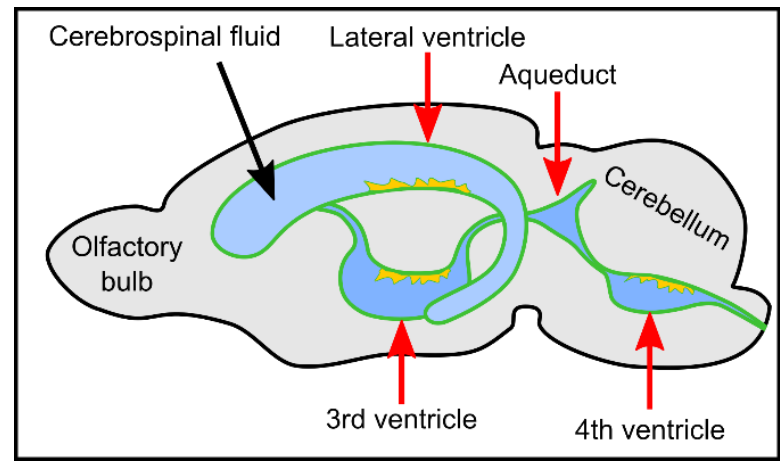

Figure 5: Sagittal view of the ventricular system of the murine brain.

The ventricular system consists of two lateral ventricles, the $3 \mathrm{rd}$ ventricle, the 4 th ventricle, and the aqueduct (red arrows), that are lined by multiciliated ependymal cells (green). MCCs are responsible for circulation of the cerebrospinal fluid (blue) produced by multiciliated CP epithelial cells (yellow). Adapted from (Wildung et al., 2018).

\subsection{Regulation of multiciliogenesis - from centrioles to cilia}

Multiciliogenesis is the process of the formation of multiple motile cilia that is precisely regulated at the transcriptional and post-transcriptional level. However, the complexity of this regulatory network is not fully understood yet. Briefly, the onset of multiciliogenesis requires the exit of the cells from the cell cycle to free the centrioles and allow for their multiplication. Upon multiplication, the centrioles are modified and migrate to the apical site of the membrane to become BBs that nucleate the ciliary axoneme. The subsequent elongation of the axoneme requires the transport of ciliary proteins from the cytoplasm to the ciliary tip, which is achieved by the intraflagellar transport (IFT) (Spassky and Meunier, 2017). IFT is not only responsible for the ciliary assembly but also for the disassembly by transporting cargos back into the cytoplasm. Upon ciliary disassembly, BBs are freed and converted to centrioles, thus allowing the cells to re-enter the cell cycle (Ishikawa and Marshall, 2011). The different steps involved in multiciliogenesis and their regulation are described below in more detail.

\subsubsection{Centriole/basal body biogenesis}

To nucleate multiple motile cilia, biogenesis of many centrioles/BBs is required. Centriole expansion occurs via a poorly understood de novo pathway, also known as the deuterosome dependent pathway (acentriolar pathway). In this pathway, electron dense granules called deuterosomes serve as a platform for centriole nucleation. In contrast, 
formation of primary monocilia requires the production of only one BB via the mother centriole dependent pathway (centriolar pathway) (Dawe et al., 2007). In BBs as well as in centrosomes, centrioles serve as microtubule-organizing platforms to enable the formation of cilia and mitotic spindles, respectively. These two mutually exclusive functions of centrioles require a precise regulation achieved by centriolar modifications yielding different accessory structures (Avidor-Reiss and Gopalakrishnan, 2013).

One crucial modification during BB conversion from centrioles is the removal of the centriolar coiled-coil protein $\underline{110} \mathrm{kDa}(\mathrm{CP} 110)$ from the distal end of the mother centriole. Thus, CP110 acts as a suppressor of primary and motile cilia assembly (Tsang and Dynlacht, 2013; Song et al., 2014). However, it has been shown that CP110 also localizes to cilia-forming BBs and rootlets, where it is involved in the formation of the ciliary adhesion complex required for correct BB anchorage. Thus, besides being a repressor of ciliogenesis at early steps, at later ciliogenic events CP110 promotes ciliogenesis (Walentek et al., 2016; Yadav et al., 2016). Due to these two opposing roles of CP110 in ciliogenesis, its levels must be tightly and timely regulated, for instance by microRNAs (miRNAs, miRs) of the miR34/449 family (Song et al., 2014) (described in section 1.3.5.2).

Following expansion and modification, centrioles migrate towards the apical cell surface, where they dock to become BBs. The process of centriole migration is not fully resolved. However, evidence indicate that the cytoskeleton with its actin-myosin network facilitates this migration (Dawe et al., 2007). The subsequent anchorage of the BB to the plasma membrane is promoted by the formation of a dense cortical actin meshwork (Avasthi and Marshall, 2012). Apical actin formation is facilitated by members of the miR-34/449 family, which repress the small guanosine triphosphate hydrolyzing enzyme (GTPase) R-RAS (Chevalier et al., 2015). Upon proper BB docking, ciliary axoneme extension occurs (Avasthi and Marshall, 2012).

\subsubsection{Ciliary assembly is mediated by intraflagellar transport}

The assembly of the axoneme depends on the transport of proteins from the cytoplasm to the ciliary tip, since cilia cannot produce its own proteins owing to the lack of ribosomes. The cargo transport along the axoneme requires IFT. IFT is a bidirectional, molecular motordriven process that is mediated by two multiprotein complexes termed IFT-A and IFT-B (Figure 28c). These two IFT particles connect the cargos to different microtubule-based molecular motors. The molecular motor for the transport of cargos bound to IFT-B particles to the ciliary tip (anterograde) is powered by kinesin-2 motors. Once the ciliary axoneme is established, it remains highly dynamic at steady state. The dynamic state occurs due to the constant removal and synthesis of ciliary proteins including the $\alpha$-and $\beta$-tubules. Ciliary turnover products are returned to the ciliary base by IFT-A particles that are driven by dynein-2 motors (retrograde). Thus, the IFT system regulates ciliary assembly and turnover, 
which thereby helps to determine the length of the cilia at steady state (Ishikawa and Marshall, 2011). Therefore, IFT is required for the maintenance of functional cilia.

\subsubsection{Ciliary disassembly}

Ciliary disassembly is a pre-requisite for cell cycle re-entry and hence occurs prior to cell proliferation. As cells represent a convenient model system to study primary cilia assembly and disassembly in a controlled manner, a large volume of work has been carried out using cell culture systems. The assembly of primary cilia in vitro can be induced by serum starvation and subsequent disassembly through the addition of serum or growth factors, which activate Aurora kinase $\underline{A}$ (AURKA) at the BB (Goto et al., 2013; Sánchez and Dynlacht, 2016). AURKA induces ciliary disassembly, at least in part, through the activation of histone deacetylase $\underline{6}$ (HDAC6) by phosphorylation (Figure 6).
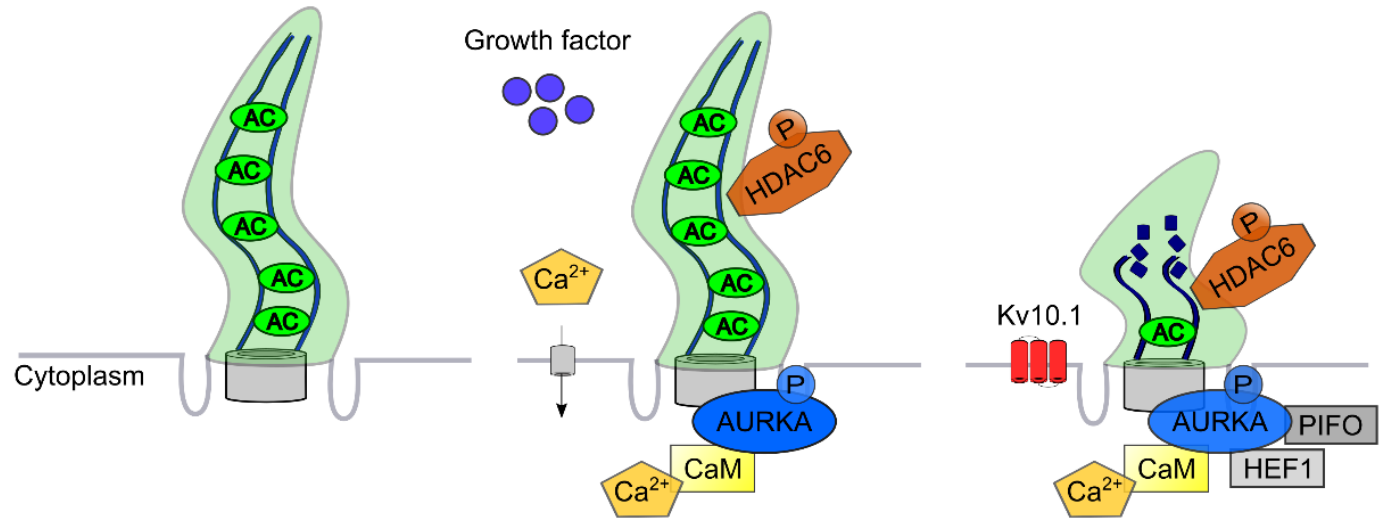

Figure 6: Model illustrating primary cilia disassembly. Upon growth factor stimulation, the $\mathrm{Ca} 2+/ \underline{c}$ almodulin (CaM) complex together with Pitchfork (PIFO) and human enhancer of filamentation 1 (HEF1) activate AURKA, which in turn activate HDAC6. HDAC6 promotes deacetylation of axonemal tubulins, thereby triggering ciliary disassembly. In addition, the voltage-gated potassium channel Kv10.1 induces primary cilia disassembly (Sánchez et al., 2016). Adapted from (Goto et al., 2013).

Briefly, growth factor stimulation and inflammatory signals trigger the release of calcium from the extracellular space and/or endoplasmic reticulum into the cytoplasm (Plotnikova et al., 2012; Zhang et al., 2014). The intracellular calcium in turn, binds to calmodulin (CaM), which then jointly activate a cascade of proteins driving ciliary disassembly. The first protein in this cascade is AURKA, which gets activated by autophosphorylation at Thr288 upon $\mathrm{Ca}^{2+} / \mathrm{CaM}$ binding (Pan et al., 2004; Plotnikova et al., 2012). However, this activation of AURKA is only transient. Hence, additional stimulation is required, which is achieved by the binding of human enhancer of filamentation 1 (HEF1) and Pitchfork (PIFO) to AURKA (Pugacheva et al., 2007; Kinzel et al., 2010). At the next step, the elevated catalytic activity of AURKA induces the phosphorylation of HDAC6 leading to HDAC6-mediated deacetylation of axonemal $\alpha$-tubulins. Deacetylation of $\alpha$-tubulins destabilizes the ciliary axoneme and thereby triggers ciliary disassembly (Pugacheva et al., 2007; Ran et al., 
2015). Not only $\alpha$-tubulin but also cortactin is a substrate of HDAC6. Deacetylation of cortactin by HDAC6 triggers its interaction with filamentous F-actin resulting in actin polymerization, which is another inducer of ciliary disassembly (Ran et al., 2015).

It has also been shown that the potassium voltage-gated channel subfamily $\mathrm{H}$ member 1 (Kv10.1, KCNH1) promotes primary cilia disassembly via a not yet identified mechanism. One proposed mechanism is that Kv10.1 increases $\mathrm{K}^{+}$permeability, which results in a hyperpolarization of the membrane, which in turn triggers $\mathrm{Ca}^{2+}$ entry into the cell leading to AURKA ativation (Sánchez et al., 2016).

Breakdown products arising during axoneme disassembly are tagged by the ubiquitin system and are recognized by the retrograde IFT trains. This allows their transport back into the cytoplasm (Huang et al., 2009a). The fate of ubiquitinated ciliary proteins is not yet fully understood. One study hypothesizes that these proteins will be reused to build a new cilium (Huang et al., 2009a). Another study showed that HDAC6 binds to these ubiquitinated ciliary breakdown products and delivers them to autophagolysosomes, where they are degraded or recycled (Kawaguchi et al., 2003; Lam et al., 2013).

After AURKA/HDAC6-mediated primary cilia resorption and subsequent cell cycle re-entry, repression of cilia regeneration is required during cell cycle progression. This inhibition is also mediated by AURKA, which then localizes to the centrioles, where it is activated by the trichoplein-nuclear distribution element-like 1 pathway (Inoko et al., 2012; Inaba et al., 2016).

So far, most studies have investigated the regulation of the disassembly of primary cilia (Goto et al., 2013; Liang et al., 2016). Thus, little is known about the mechanisms triggering motile cilia disassembly except one study conducted by Lam and colleagues (Lam et al., 2013). They describe the shortening of cilia upon CS exposure to be mediated by an HDAC6-dependent autophagy pathway. In brief, apart from its role as a deacetylase, HDAC6 contains a ubiquitin-binding as well as a dynein-interacting domain. The ubiquitinbinding domain of HDAC6 interacts with ubiquitinated ciliary compounds. The dyneininteracting domain of HDAC6 interferes with the cytoplasmatic dynein motor proteins, thereby facilitating the transport of ubiquitinated ciliary proteins via the cytoplasmatic microtubule network to the aggresomes, where ciliary proteins are degraded upon CS exposure (Lam et al., 2013). Since CS-mediated repression of the deacetylase Sirtuin-1 (Rajendrasozhan et al., 2008; Yao et al., 2012) inhibits the deacetylase activity of HDAC6 by increasing its acetylation (Liu et al., 2012; Gal et al., 2013; Lam et al., 2013), it is still likely that HDAC6 can regulate motile cilia disassembly via its deacetylase activity in the absence of CS. 


\subsubsection{Cell cycle-associated functions of Aurora family members}

In mammals, the Aurora family of serine/threonine kinases consists of AURKA, Aurora B, and Aurora C. Besides its role in primary cilia disassembly and repression of cilia regeneration (Pugacheva et al., 2007; Plotnikova et al., 2012), AURKA is a well-known centrosomal kinase involved in mitosis at different steps. Mitotic functions of AURKA include the regulation of mitotic entry, centrosome maturation and separation, and bipolar spindle formation. Although Aurora B shares high similarity with AURKA in protein sequence and structure, its functions in cell cycle progression differs, most likely due to the recruitment of other cofactors. The regulation of chromosome-microtubule interactions, chromatid cohesion, spindle stability, and cytokinesis belong to the functions of Aurora B. The third Aurora family member is Aurora $\mathrm{C}$, which represents an exception due to its predominant expression in male gametes, where it is involved in meiosis (Carmena et al., 2009).

\subsubsection{Transcriptional regulation of multiciliated cell differentiation}

The decision of a cell to exit the cell cycle and subsequently form a motile cilium is highly regulated at the transcriptional and post-transcriptional level (Figure 7). The first step required for the initiation of $\mathrm{MCC}$ differentiation is the inhibition of Notch signaling, which leads to the activation of two master regulators, Geminin coiled-coil domain containing

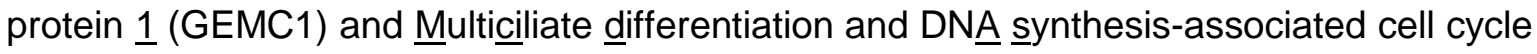
protein (MCIDAS or Multicilin). GEMC1 as well as MCIDAS interact with E2F transcription factor 4 and $5(E 2 F 4 / 5)$ to activate a regulatory network of downstream effectors.

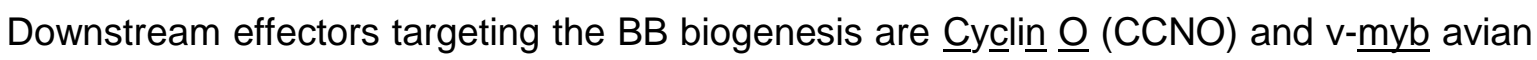
myeloblastosis viral oncogene homolog (MYB). Later steps such as BB docking, axoneme assembly, and cilia motility are regulated by forkhead box $\underline{\mathrm{J} 1}$ (FOXJ1) and regulator factor $\underline{X 2}$ and X3 (RFX2, RFX3) (Spassky and Meunier, 2017). Recently, we and others identified TAp73 as a central regulator of airway multiciliogenesis since it induces the expression of several pro-ciliogenic factors necessary for motile cilia formation (see section 1.3.4.2 and 0 for detailed information) (Marshall et al., 2016; Nemajerova et al., 2016). 


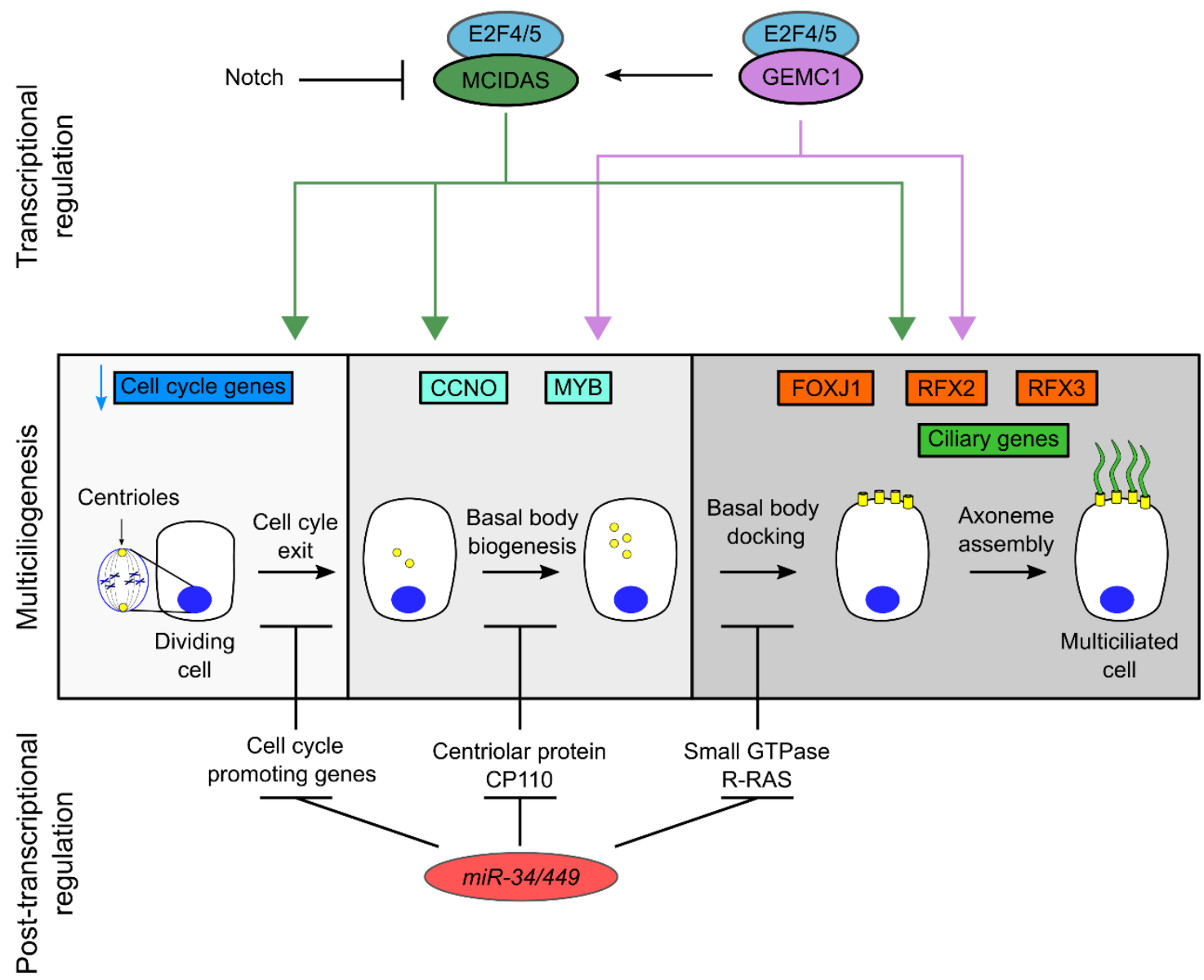

Figure 7: Transcriptional and post-transcriptional regulation of multiciliogenesis.

The onset of multiciliogenesis requires the inhibition of Notch signaling. At the transcriptional level, GEMC1 and MCIDAS, both bound to E2F4/5, regulate cell cycle exit, BB biogenesis and docking, and axoneme assembly by targeting different pro-ciliogenic factors such as CCNO, MYB, FOXJ1, RFX2, and RFX3. At the post-transcriptional level, the miR-34/449 family members repress cell cycle promoting genes, CP110, and $R-R A S$ and thereby promoting cell cycle exit, BB biogenesis, and BB docking. Adapted from (Nemajerova et al., 2016).

\subsubsection{Master regulators of multiciliogenesis - GEMC1, MCIDAS, and E2Fs}

GEMC1 and MCIDAS, together with Geminin, belong to the Geminin superfamily and represent central regulators of multiciliogenesis (Stubbs et al., 2012; Arbi et al., 2016; Terré et al., 2016). When bound to E2F4/5, GEMC1 transcriptionally activates a wide range of pro-ciliogenic factors regulating BB multiplication (MYB, CCNO) and cilia formation (FOXJ1, RFX3). Thus, the deficiency of Gemc1 in mice causes defects associated with impaired multiciliogenesis such as male and female infertility, reduced generation of multiple motile cilia as well as hydrocephalus (Arbi et al., 2016; Kyrousi et al., 2016; Terré et al., 2016).

The second most upstream regulator of multiciliogenesis is MCIDAS, which is sufficient and necessary to promote multiciliogenesis. Mcidas is flanked in the genome by two other prociliogenic factors, namely Ccno and Cdc20b (host gene of miR449). Due to the lack of a DNA-binding domain, MCIDAS elicits its function by acting as a cofactor for E2F4/5 to induce a similar transcriptional program as GEMC1 (e.g. TAp73, FOXJ1, RFX2, RFX3, and MYB) (Stubbs et al., 2012; Boon et al., 2014; Chung et al., 2014; Ma et al., 2014; 
Nemajerova et al., 2016). The importance of MCIDAS in regulating multiciliogenesis is highlighted by the fact that mutations in the MCIDAS gene cause a disease termed reduced generation of multiple motile cilia in humans (Boon et al., 2014).

Both MCIDAS and GEMC1 form a complex with E2F4/5 to activate transcription of prociliogenic factors. E2F4/5 belong to the E2F family, which consists of well-known transcriptional regulators of the cell cycle (Trimarchi and Lees, 2002). While E2F4/5 repress cell cycle genes and therefore promote cell cycle exit (Trimarchi and Lees, 2002), upon binding to MCIDAS or GEMC1, E2F4/5 activate the transcription of pro-ciliogenic genes (Ma et al., 2014). Deficiency of either E2f4 or E2f5 in mice causes defects related to impaired multiciliogenesis but with distinct phenotypes. E2f4 knockout (KO) mice develop defects in airway cilia with no hydrocephalus (Danielian et al., 2007), whereas E2f5 KO mice display hydrocephalus (Lindeman et al., 1998). Neither KO mouse strain is infertile, possibly due to their redundant functions. Infertility is only triggered by combined loss of E2f4 and E2f5 due to defective ED cilia (Danielian et al., 2016).

\subsubsection{P73 - a novel central regulator of multiciliogenesis}

We identified TAp73 as a central regulator of airway multiciliogenesis (Marshall et al., 2016; Nemajerova et al., 2016). In this chapter, background information regarding the structure of the transformation-related protein $\underline{73}$ (Trp73) gene as well as a phenotypic description of different $p 73 \mathrm{KO}$ mouse models are listed. Regulation of multiciliogenesis by TAp73 is described in the preliminary results section 0 .

\subsection{Structure of the Trp73 gene}

P73 belongs to the p53 tumor suppressor family of transcription factors, along with p63. The Trp73 gene encodes two different p73 isoform classes, TAp73 and DNp73, each arising from a different promotor and exerting opposing cellular functions (Figure 8) (Melino et al., 2002).

\section{Trp73 gene}
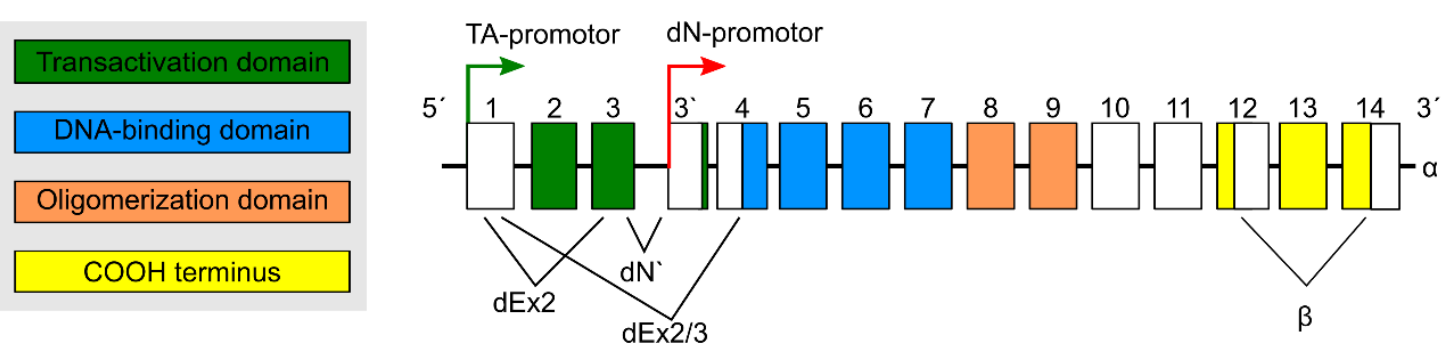

Figure 8. 
Figure 8: Gene structure of murine $\operatorname{Trp} 73$.

The Trp73 genes encodes two main different isoforms, TAp73 and DNp73, each transcribed from a different promotor. Alternative splicing of products generated from the TA-promotor give rise to distinct N-terminal truncated p73 isoforms ( $\mathrm{dEx} 2, \mathrm{dEx} 2 / 3$, and $\left.\mathrm{dN} \mathrm{N}^{\prime}\right)$. Alternative splicing at the $3^{\prime}$-end generates different transcripts, while only the most relevant ones are depicted ( $\alpha$ and $\beta$ ). Exons are displayed as boxes and colored according to their different domains. Adapted from (Tomasini et al., 2008).

TAp73 is transcribed from the extrinsic promotor P1 and acts as the transcriptional active form, since it contains the $\mathrm{N}$-terminal transactivation (TA) domain. DNp73 representing the $\mathrm{N}$-terminal truncated isoform is transcribed from the intrinsic promotor P2 and lacks the TA domain. In addition, other N-terminal truncated isoforms are generated by alternative splicing of the transcript generated from the TA-promotor (dEx2, dEx2/3, and dN') (Melino et al., 2002).

p73 isoforms act as tetramers, which consist either of homo- or heterodimers. Heterodimers are generated either with other p73 splice variants, p63 or to a lesser extent with p53. The TAp73 homotetramer transcriptionally activates proapoptotic genes and thereby acts as a tumor suppressor, while the DNp73 isoforms are incapable of triggering gene expression directly, as this isoform lacks the TA domain. However, DNp73 exhibits a dominant negative effect on TAp73, which leads to the inhibition of the proapoptotic activity of TAp73. Thus, DNp73 indirectly inhibits apoptosis and acts as an oncogene. The inhibitory function of DNp73 is mediated either by the formation of heterodimers with TAp73 and thereby sequestering TAp73 or by competing with the proapoptotic protein p53 for the same promotor binding sites. Therefore, DNp73 regulates the activity of both proapoptotic proteins (TAp73, p53). Moreover, TAp73 induces DNp73 expression, which creates a dominant negative feedback loop (Melino et al., 2002).

Besides the $\mathrm{N}$-terminal p73 isoform classes generated by alternative promoters (TAp73, DNp73), alternative C-terminal (3'-end) splicing also occurs yielding at least seven distinct C-terminal isoforms ( $\alpha, \beta, \gamma, \delta, \varepsilon, \zeta$, and $\eta$ ) (Figure 8). p73 $\alpha$ is the most abundant isoform under physiological conditions and represents the longest isoform containing a fully functional sterile alpha motif (SAM) domain. All the other C-terminal isoforms lack the SAM domain. Current data suggest that the SAM domain contributes to the stability of p73 tetramer, thereby influencing p73 transcriptional activity. In contrast, it has been shown that p73 $\beta$ is the strongest proapoptotic transcriptional activator (Vikhreva et al., 2018).

\subsection{Mouse models of $\mathrm{p73}$}

For the identification of the function of p73 during development, different mouse models have been generated: global $p 73 \mathrm{KO}$ mice and isoform specific $\mathrm{KO}$ strains for TAp73 and DNp73. P73 KO mice deficient for TAp73 as well as DNp73 possess a deletion of exon 5 and 6 in the DNA binding domain, yielding a non-functional protein (Yang et al., 2000). TAp73 KO mice lack exon 2 and 3, which encode the TA domain, while DNp73 isoforms 
can still be expressed using the intrinsic P2 promotor (Tomasini et al., 2008). In DNp73 KO mice, the alternative 3' exon is depleted from the gene locus (Tissir et al., 2009; Wilhelm et al., 2010).

Analyses of the different $p 73 \mathrm{KO}$ mouse models showed that the functions of p73 are complex. Therefore, $p 73$ deficiency results in various phenotypes characterized by infertility as well as neurological and immunological defects (Yang et al., 2000; Tomasini et al., 2008; Tomasini et al., 2009; Wilhelm et al., 2010; Holembowski et al., 2014; Inoue et al., 2014). P73 and TAp73 KO mice are infertile (Yang et al., 2000; Tomasini et al., 2008), while DNp73 $\mathrm{KO}$ mice are fertile (Wilhelm et al., 2010). Hence, the infertility phenotype is attributed primarily to the loss of the TAp73 isoform. Poor quality of eggs caused by impaired meiosis contributes to female infertility in TAp73 KO mice. Moreover, oocytes from TAp73 KO mice did not reach the FT, since they were trapped in the bursa (Tomasini et al., 2008; Tomasini et al., 2009). Male infertility in TAp73 KO mice is associated with defective spermatogenesis, as TAp73 $\mathrm{KO}$ mice possess an increased apoptotic rate in spermatogonia, reduced adhesion of germ cells to Sertoli cells, and malformed spermatids. Consequently, the seminiferous tubules of the testes are nearly empty. However, a few spermatozoa can be found in the testes (Holembowski et al., 2014; Inoue et al., 2014). In this study, we identified a new additional mechanism contributing the infertility phenotype in male and female TAp73 KO mice (described in 4.1.2 and 4.1.3).

The neuronal phenotype differs between the three $p 73 \mathrm{KO}$ mouse models. $P 73 \mathrm{KO}$ mice develop the strongest phenotype, including a variety of defects such as neuronal degeneration (cortical hypoplasia), hippocampal dysgenesis, and hydrocephalus. The neuronal degeneration phenotype has been primarily attributed to the loss of DNp73 due to two reasons. Firstly, a similar neurodegeneration pattern was observed in $D N p 73$ and $p 73$ $\mathrm{KO}$ mice but not in TAp73 KO mice (Tissir et al., 2009; Wilhelm et al., 2010). Secondly, it has been shown that DNp73 promotes the survival of mature neurons by counteracting TAp73-mediated apoptosis (Pozniak, 2000; Pozniak et al., 2002; Talos et al., 2010). The hippocampal dysgenesis defects of $p 73 \mathrm{KO}$ mice are also observed in TAp73 KO mice but not in DNp73 KO mice, implicating that TAp73 is the essential isoform for hippocampal development (Tomasini et al., 2008). Of note, severe hydrocephalus is only observed in p73 KO mice (Yang et al., 2000). In addition, p73 KO mice display ependymal denudation and loss of ependymal cilia. Thus, p73 ensures ependyma maintenance and thereby helps in regulating cerebrospinal fluid homeostasis (Medina-Bolívar et al., 2014). In sum, both p73 isoforms contribute to different steps in the neuronal development as phenotypes from the isoform-specific KOs are clearly different. However, TAp73 and DNp73 may also possess some overlapping functions, since the p73 KO phenotype is much stronger than the phenotype of the isoform-specific KOs. 
Besides infertility and impaired neuronal development, p73 KO mice are susceptible to infections that lead to chronic inflammation due to defective airway multiciliogenesis (described in 1.6). Due to all these impairments, most of the $p 73 \mathrm{KO}$ mice die within the first month (Yang et al., 2000). By contrast, TAp73 KO mice have a only slightly reduced life span, while the DNp73 KO mice possess a normal life span (Tomasini et al., 2008; Wilhelm et al., 2010).

\subsubsection{Downstream effectors of multiciliogenesis MYB and CCNO}

$\mathrm{BB}$ biogenesis is regulated by both MYB and CCNO. The transcription factor MYB has three paralogs in mice: MYBL1, MYBL2, and MYB (in mammals: A-MYB, B-MYB, and C-MYB, respectively). MYB has been shown to regulate BB amplification (Tan et al., 2013). Ccno, found in the same genomic region as the other pro-ciliogenic factors (miR449 (Lizé et al., 2010b; Lizé et al., 2010a; Song et al., 2014)]) and Mcidas (Stubbs et al., 2012; Boon et al., 2014)]) is also necessary for de novo BB biogenesis (Funk et al., 2015). Mutations in CCNO are observed in patients with the reduced generation of multiple motile cilia disorder (Wallmeier et al., 2014).

\section{FOXJ1 and RFX factors}

FOXJ1 belongs to the forkhead box family of transcription factors and is expressed in ciliated cells of multiciliated tissues (Hackett et al., 1995; Murphy et al., 1997; Blatt et al., 1999). In MCCs, FOXJ1 activates the transcription of a variety of genes required for BB docking and several subsequent steps essential for the formation of motile cilia. Examples of FOXJ1 targets are genes required for IFT or IDA/ODA assembly. Hence, depletion of Foxj1 in mice results in the complete loss of ciliary axonemes associated with an impaired BB docking, while the production of BB is not influenced (Chen et al., 1998; Brody et al., 2000; Gomperts et al., 2004; You et al., 2004).

Other important downstream effectors of multiciliogenesis are the transcription factors RFX2 and RFX3. They belong to the RFX family consisting of eight members, while only RFX2-4 are cilia-associated transcription factors. RFX2 and RFX3 activate multiple cilia genes, which regulate ciliary assembly (e.g. IFT proteins) and cilia motility (e.g. dyneins). Due to their partially redundant functions, deficiency of cilia-associated RFX factors in mice only results in a mild ciliary phenotype (Chung et al., 2012; Choksi et al., 2014; Chung et al., 2014). 


\subsubsection{Post-transcriptional regulation of multiciliated cell differentiation}

\subsubsection{1 miRNA biogenesis and mechanism of action}

miRNAs represent a class of small noncoding ribonucleic acid (RNA) sequences that regulate gene expression post-transcriptionally. Post-transcriptional regulation by miRNAs is achieved by its interaction with specific target mRNA sequences resulting in translational repression or mRNA degradation. Biogenesis of the 20-25 nucleotide long miRNAs occurs in the following steps:

1) Transcription of the miRNA gene by RNA polymerase II yielding primary miRNAs folded into hairpin structures.

2) Cleavage of primary miRNAs by the Drosha-Pascha (DGCR8 in mammals) complex into approximately 70-nucleotide long precursor miRNAs (pre-miRNAs).

3) Nuclear export of pre-miRNAs into cytoplasm by the Ran-GTP dependent transport receptor exportin-5.

4) Cleavage of pre-miRNAs into mature miRNA duplexes by Dicer and its cofactor HIV1 trans-activating response RNA binding protein.

5) Incorporation of one single mature miRNA strand into the multimeric protein-RNA complex called miRNA-induced silencing complex. This complex contains proteins of the Argonaute family that function in miRNA repression and endonucleolytic cleavage of the mRNA, whereas Argonaute 2 is the only family member that can cleave mRNAs. In most cases, the other miRNA strand is degraded.

Within the miRNA-induced silencing complex, miRNAs bind to their mRNA target sequence, which is commonly located within the 3' untranslated region (UTR) of the mRNA. The 6 to 8 nucleotide long sequence of miRNAs that usually binds with imperfect complementarity to target mRNAs is called the "seed sequence" and is located near the 5' terminus (Bartel, 2018). Thus, the seed sequence of the miRNAs is used to predict targets. One individual miRNA can target multiple mRNAs (Brennecke et al., 2005) and each mRNA in turn can be controlled by several miRNAs (Doench and Sharp, 2004). miRNAs repress their target transcripts through two different mechanisms, depending on the degree of miRNA-mRNA complementarity. Perfect complementarity and presence of the Argonaute 2 protein trigger slicing of the mRNA (as observed in siRNA-mediated gene regulation). In plants, this slicing mode of repression is commonly observed for miRNAs. In contrast, metazoan miRNAs bind in most cases with imperfect complementarity to their target mRNA and the repression occurs without slicing the mRNA. The repression of metazoan mRNAs is mediated via deadenylation-dependent target mRNA decapping. One important mediator of this process is trinucleotide repeat containing $\underline{6}$ (TNRC6), which recruits deadenylase complexes such as CCR4-NOT. In addition to its deadenylase activity, CCR4-NOT recruits $\underline{D} E A \underline{D}-$ box helicase $\underline{6}(\mathrm{DDX} 6)$ and elF4E-Transporter (4E-T), which causes the inhibition of protein 
translation initiation. Thus, miRNAs can destabilize target mRNAs but can also inhibit protein translation (Bartel, 2018).

\subsubsection{The $m i R-34 / 449$ family}

The miR-34/449 family is strongly conserved in vertebrates and encodes six homologous miRNAs (miR34a,b,c and miR449a,b,c) derived from three different genomic loci. They are classified as one family due to their identical seed sequences and common targets (Figure 9a) (Bao et al., 2012b; Song et al., 2014).

a

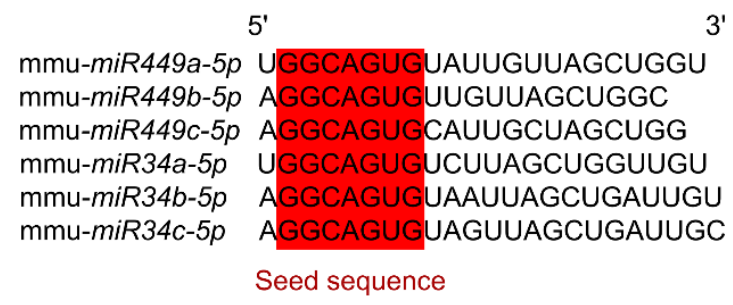

b

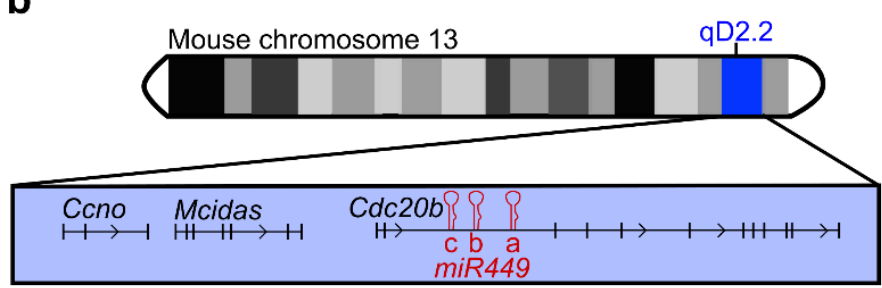

Figure 9: Seed sequence and genomic location of $m i R-34 / 449$ family members.

a) miR-34/449 family members possess the same seed sequence (red box). b) miR449 is located in the second intron of cell division cycle $\underline{20 \mathrm{~b}}$ (Cdc20b) on murine chromosome 13 , which also harbors the genomic regions of Ccno and Mcidas. Adapted from (Gallinas and Lizé, 2013).

In mice, miR34b and miR34c (miR34b,c from here on) are transcribed from one common transcript of chromosome 9qA5.3 (in human: 11q23.1), located within the Btg AntiProliferation factor $\underline{4}$ gene (Btg4). In contrast, murine miR34a is encoded separately on chromosome 4qE2 (in humans 1q36.22) (Hermeking, 2010). The miR449 cluster consisting of miR449a, $b$, and $c$ (miR449 from here on) is located in the second intron of cell division cycle $20 b$ (Cdc20b) on chromosome 13qD2.2 in mice (5q11.2 in humans) and shares its promoter (Figure 9b) (Lizé et al., 2010b) .

The miR34 and miR449 cluster are induced by p53 and E2F1, respectively, both of which are two important DNA damage-responsive transcription factors (Yang et al., 2009; Hermeking, 2010; Lizé et al., 2010b; Lizé et al., 2011). In addition, members of the miR$34 / 449$ family inhibit the activity of the retinoblastoma protein $(R b)-E 2 F 1$ pathway indirectly e.g. by repressing CDK6 and CDC25A (Yang et al., 2000; Lizé et al., 2010b; Lizé et al., 2011). Although it has not been shown experimentally, it can be assumed that both miRNAs also repress E2F1 directly, as it is known that miR449 directly represses E2F2 and E2F3 
(Bao et al., 2012b), which are highly homologous to E2F1 (Trimarchi and Lees, 2002). Moreover, miR-34/449 family members promote p53 activity by increasing its acetylation (e.g. via Sirtuin-1 repression) and thereby facilitating the crosstalk between p53 and E2F1 (Lizé et al., 2010b; Lizé et al., 2011). The attenuation of E2F1 along with the activation of p53 induces cell cycle arrest or apoptosis. Thus, miR-34/449 family members act as tumor suppressors and are therefore often downregulated in various cancer entities (Yang et al., 2000; Hermeking, 2010; Lizé et al., 2010b; Lizé et al., 2011).

Cell cycle arrest is also necessary for the onset of multiciliogenesis and thus, miR-34/449 family members are expressed in multiciliated organs and induced during mucociliary differentiation (Lizé et al., 2010a; Marcet et al., 2011). Depletion of all miR-34/449 family members in mice results in severe respiratory dysfunctions caused by defective mucociliary clearance, early death from respiratory failure, and infertility (Song et al., 2014; Wu et al., 2014; Otto et al., 2017). Different mechanisms on how miR-34/449 regulates multiciliogenesis have been described. For example, in MCCs miR-34/449 initiates cell cycle exit and entry into MCC differentiation by repressing cell cycle promoting genes (Lizé et al., 2010b; Lizé et al., 2011; Otto et al., 2017) (Figure 7). It has also been proposed that miR449 triggers multiciliogenesis by directly repressing Notch signaling. The validation of Notch signaling as a target of miR449 was performed in xenopus embryonic epidermis (Marcet et al., 2011). However, in the airway epithelium of miR-34/449 $\mathrm{KO}$ mice the deregulation of the Notch pathway could not be confirmed (Otto et al., 2017). Thus, regulation of Notch signaling by miR-34/449 might be species-dependent. In addition, miR$34 / 449$ promote multiciliogenesis at later steps by repressing Cp110 (Song et al., 2014) and $R$-ras (Chevalier et al., 2015), thereby facilitating BB maturation and docking respectively (Figure 7). However, Cp110 remains a controversial miR-34/449 target as Otto and colleagues could not reproduce the observed upregulation of $C p 110$ transcript and protein levels in the respiratory epithelium of miR-34/449 KO mice (Otto et al., 2017).

Of note, miR34a represents an exception, as it is not specifically expressed in multiciliated tissues and not upregulated during mucociliary differentiation (Lizé et al., 2010a; Song et al., 2014). Moreover, miR34b,c x miR449 KO mice yield the same respiratory and infertility phenotype as miR34a,b,c x miR449 KO mice. Thus, the presence of miR34a did not rescue the ciliogenesis defects in mouse models of miR-34b,c/449 depletion. In contrast, one allele of miR34b,c or miR449 was sufficient to rescue proper multiciliogenesis (Song et al., 2014). Hence, miR34b,c and miR449, but not miR34a, seem to be the main drivers for multiciliogenesis regulation. However, the contribution of a single miRNA of the miR-34/449 family to multiciliogenesis has not been studied in detail so far. Thus, in this thesis, the involvement of miR449 alone in mucociliary differentiation and lung homeostasis was addressed (see section 4.2). 


\subsection{Diseases associated with motile cilia dysfunction}

Dysfunction of motile cilia is associated with several disorders, which can be both inherited and acquired. One classical inherited disorder primarily associated with airway cilia defects is PCD. The term "primary" (primary ciliary dyskinesia) does not refer to primary cilia but is used to distinguish the genetically triggered motile cilium defects from secondary, acquired motile cilia disorders. Acquired cilia disorders are often characterized by airway mucociliary dysfunction, which mainly occurs due to exposure to environmental pollutants e.g. cigarette smoke (CS) or diesel exhaust particles (DEPs). Mucociliary clearance defects increase the susceptibility to recurrent respiratory infections (exacerbations), thereby triggering chronic lung diseases such as COPD (Tilley et al., 2015).

\subsubsection{Primary ciliary dyskinesia - Inherited cilia dysfunction}

PCD is an autosomal-recessive inherited disorder of motile cilia leading to frequent upper and lower respiratory tract infections. Respiratory tract infections result from a defective airway ciliary function leading to a highly dyskinetic cilia beating, which in turn impairs mucociliary clearance. So far, mutations in more than 30 genes encoding proteins associated with cilia structure or function have been linked to PCD. Components of the ODA such as $\underline{d} y n e i n \underline{a x o n e m a l ~ h e a v y ~} \underline{\text { chain }} \underline{5}$ (DNAH5) and $\underline{\text { dynein }}$ axonemal intermediate chain 1 (DNAI1) belong to the most frequently mutated genes found in PCD patients (Milla, 2016). Besides respiratory cilia, motile cilia of other organs are affected in PCD as well. Hence, clinical manifestations of PCD are heterogenous and include male infertility, situs inversus, congenital heart disease, polycystic kidney disease, and in rare cases hydrocephalus. Of note, male infertility caused by defective sperm motility occurs frequently, whereas only some PCD-affected females show subfertility or ectopic pregnancies where the implantation of the zygote takes place in the FT (Goutaki et al., 2016; Vanaken et al., 2017).

\subsubsection{Chronic obstructive pulmonary disease - Acquired cilia dysfunction}

COPD is the third leading cause of death world-wide with increasing prevalence, morbidity, and mortality, especially in the elderly population. A major characteristic of COPD is the ongoing inflammation in the lung, which results in airflow limitation that is linked to a decline in lung function. The inflammatory response is triggered upon exposure to noxious gases such as CS, air pollution, and job-related fumes. Hence, harmful gases represent the main risk factors for COPD. In addition, respiratory infections in childhood and genetic determinants (e.g. deficiency of the antiprotease $\alpha 1$-antitrypsin) are also associated with increased COPD susceptibility. Chronic inflammation in the lungs and subsequent abnormal repair and regeneration result in the destruction of the airway and alveolar compartment, which leads to chronic bronchitis at one end and emphysema at the other end. Most COPD patients present both features. In addition, repeated exacerbations together with the 
ongoing inflammation shorten the time required for proper tissue repair and regeneration. Exacerbations defined as the worsening of symptoms are mainly triggered by respiratory viral and bacterial infections, which intensify the inflammation. Thus, exacerbations increase the tissue injury, thereby promoting disease progression and severity, which results in a worse prognosis and repeated hospital admissions (Barnes et al., 2015).

Chronic bronchitis is characterized by airway inflammation and mucus secretion and represent one hallmark of COPD. Excessive mucus production occurring in response to inflammatory mediators is caused by goblet cell metaplasia as well as increased mucin synthesis. Mucus hypersecretion triggers airway mucociliary dysfunction, which is intensified by an impaired transport of mucus out of the airways. Under healthy conditions, mucus is transported by the coordinated beating of cilia. However, upon inflammation, cilia are damaged and thus, mucus transport is hampered leading to the accumulation of respiratory pathogens such as Haemophilus influenzae in the airways (Rogers, 2005). Haemophilus influenzae, an opportunistic bacterium commonly found to be accumulated in the lower airways of COPD patients, is known to reduce cilia beat frequency (Johnson and Inzana, 1986; Janson et al., 1999; Bailey et al., 2012). Many other pathogens such as pseudomonas (Wilson et al., 1985; Read et al., 1992), fungi (Piecková and Jesenská, 1996; Bafadhel et al., 2014), and viruses (Zhang et al., 2005) have developed ciliostatic factors that abrogate mucociliary clearance, which also typically colonize the lungs of COPD patients (Sethi and Murphy, 2008; Bafadhel et al., 2014).

Another hallmark of COPD is emphysema. The underlying pathogenic mechanism of emphysema is based on the accumulation of inflammatory cells in response to harmful environmental agents. Inflammatory cells secrete destructive mediators (such as matrix metalloproteinases (MMPs) or neutrophil elastase (NE), which degrade the elastin fibers leading to the destruction of the alveolar parenchyma (emphysema) (Barnes et al., 2015). Under healthy conditions, proteases are counterbalanced by antiproteases (e.g. tissue inhibitors of metalloproteinases (TIMPs) or a1-antitrypsin). However, continuous elevated levels of proinflammatory mediators result in protease-antiprotease imbalance, causing tissue damage. In COPD, NE, MMP9, and MMP12 are considered the main contributors to the pathogenesis of emphysema (Houghton, 2015).

Cilia beating is important to ensure effective mucociliary clearance. In COPD, cilia beating (Piatti et al., 2005; Yaghi et al., 2012) and cilia length (Hessel et al., 2014) are reduced. However, the latter one remains controversial as cilia length seems to be unaffected in some COPD patient cohorts (Yaghi and Dolovich, 2016). Despite these data, little is known about the underlying signaling pathways leading to cilia dysfunction in COPD as most data are descriptive and only deal with the correlation between ciliary structure and function. Moreover, the role of defects in motile cilia in initiation and progression of COPD is not well studied. Thus, more research on cilia and mucociliary dysfunction in COPD is needed to 
identify underlying mechanisms and develop therapeutic approaches to correct mucociliary dysfunction and avoid frequent exacerbations, the driver of COPD progression (Yaghi and Dolovich, 2016). The present study builds on this issue and provides a novel link between cilia maintenance and the onset of COPD. The urgent need for understanding the underlying disease process is highlighted by the fact that till date there is no treatment strategy to cure COPD. Current treatments such as bronchodilators and anti-inflammatory drugs only reduce the symptoms (Barnes et al., 2015).

\subsection{Airway epithelial regeneration - a process impaired in COPD}

The lung is a quiescent tissue under homeostatic conditions. However, upon injury it is capable of extensive repair and regeneration due to the presence of several progenitor cells. Progenitor cells of the airway epithelium are basal and club cells (described in section 1.2.1.1), which can re-enter the cell cycle and proliferate in response to epithelial damage. Upon proliferation, progenitor cells differentiate into various airway epithelial cells, thereby repopulating the lost cells. Hence, a tightly regulated balance between proliferation and differentiation is required for proper airway regeneration. As the airways are constantly exposed to noxious gases, which damage the airway epithelium, proper airway epithelial regeneration is necessary to maintain lung function. Furthermore, regeneration is important to ensure healthy lung aging. Disruption of the regeneration process leads to airway remodeling, which is one of the characteristic feature of COPD that is caused by chronic inflammation as well as repeated exacerbations (Beers and Morrisey, 2011).

\subsubsection{Murine models of airway epithelial regeneration}

Airway epithelial regeneration can be studied by using various murine models, which target distinct regions within the airways. Distal airway regeneration is primarily mediated by variant club cells that can be triggered by naphthalene treatment (Plopper et al., 1992; van Winkle et al., 1995). In contrast, the regeneration of the proximal airways can be monitored in ALI cultures, where basal cells are responsible for the reconstitution of the pseudostratified epithelium (You et al., 2002). Both model systems were applied in this study to investigate the airway epithelial regeneration potential in a genetically modified mouse strain.

\subsubsection{Naphthalene-induced distal airway regeneration}

Naphthalene is a toxic and ubiquitous air pollutant that is commonly found indoors and outdoors due to multiple emission sources. Some of the emission sources include diesel exhaust, CS, moth and pest repellents. Naphthalene is both a polycyclic aromatic hydrocarbon and a volatile organic compound. It is converted into naphthalene-1,2-epoxide, which is further metabolized into cytotoxic and reactive compounds. The initial bioactivation 
of naphthalene is mediated by cytochrome $\underline{P} 450$ (CYP) monooxygenases (Buckpitt et al., 2002). One of the main P450 monooxygenase responsible for the conversion of naphthalene in mice is CYP2F2 (human ortholog CYP2F1) (Buckpitt et al., 1995; Li et al., 2011). In the airways, club cells express high levels of CYP2F2. Thus, club cells are very sensitive for naphthalene-induced cytotoxicity. Of note, CYP2F2 is also expressed in the liver, however to a much lesser extent (Baldwin et al., 2004).

Lung injury and repair processes occurring after naphthalene exposure by intraperitoneal (i.p.) injection have been extensively studied in mice (Buckpitt et al., 2002). Naphthaleneinduced injury is characterized by a massive exfoliation of club cells occurring within the first 24 hours (h) after treatment (Figure 10) (van Winkle et al., 1995).
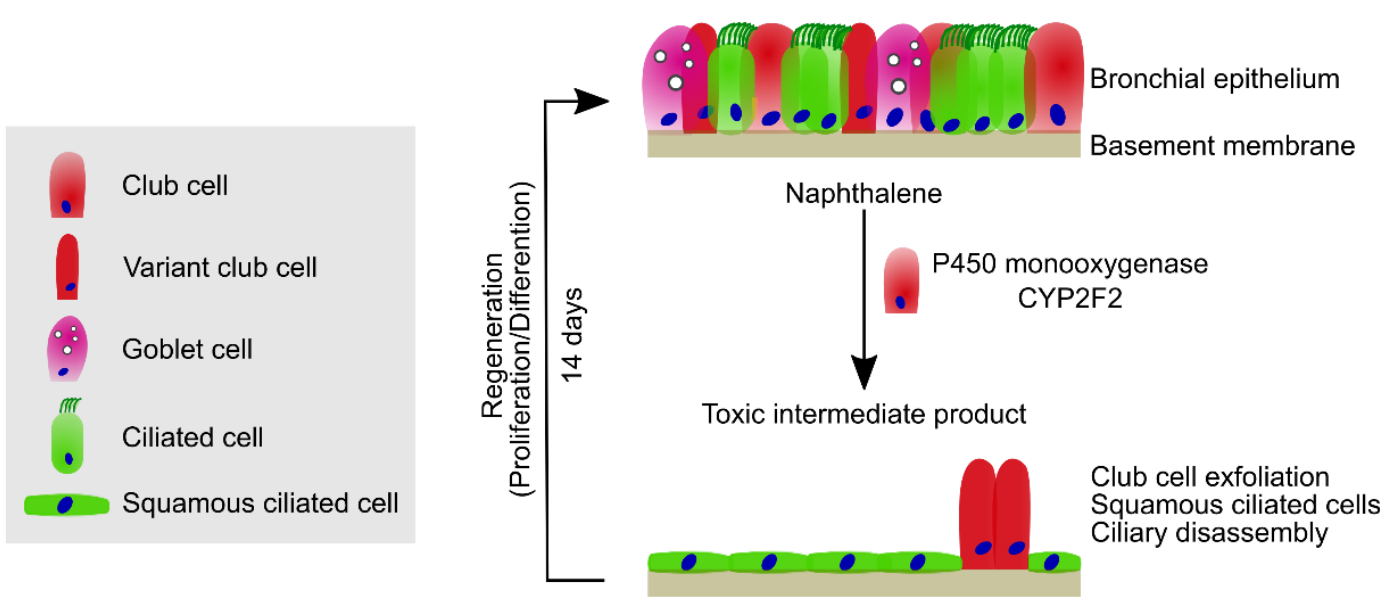

Figure 10: Naphthalene-induced airway epithelial injury.

Naphthalene is converted into a toxic intermediate product by P450 monooxygenases (mainly CYP2F2) that are specifically expressed in club cells. Thus, naphthalene treatment leads to exfoliation of injured club cells. Subsequent regeneration is mediated by ciliated cells and a small subset of surviving club cells, termed variant club cells. Ciliated cells, on the one hand, disassemble their motile cilia and spread out to cover the denuded epithelium. Variant club cells, on the other hand, proliferate and differentiate and thereby help to re-establish the pseudostratified epithelium within 14 days. Adapted from (Volckaert et al., 2011).

To cover the resulting denuded bronchial epithelium, MCCs undergo squamous metaplasia accompanied by ciliary disassembly (van Winkle et al., 1995; van Winkle et al., 1999; Lawson et al., 2002; Rawlins et al., 2007). Thus, the naphthalene model not only provides useful insights into club cell injury but also into the regulation of disassembly and assembly of motile cilia upon epithelial damage.

Upon club cell exfoliation in the distal airways, subsequent proliferation and repopulation of the airway epithelium is mediated by naphthalene-resistant club cells, termed variant club cells. Variant club cells are located in two different stem cell niches (neuroepithelial bodies and bronchoalveolar duct junction) and lack CYP2F2, rendering them naphthalene-resistant (Reynolds et al., 2000; Giangreco et al., 2002). As variant club cells represent a rare population, it is unlikely that the rapid repair after naphthalene injury is only mediated by 
variant club cells. However, the ability of MCCs to proliferate and transdifferentiate post naphthalene treatment and thereby contributing to the repair process is controversial (Park et al., 2006; Rawlins et al., 2007).

The extent of naphthalene injury is dose-dependent and differs between gender, strain, and age (Buckpitt et al., 2002). Lower naphthalene doses ( $\leq 200 \mathrm{mg} / \mathrm{kg}$ ) injure distal airways, while only higher concentrations ( $\geq 300 \mathrm{mg} / \mathrm{kg}$ ) additionally affect proximal airways (West et al., 2001). Moreover, the gender-based difference results from a distinct naphthalene metabolism between females and males rendering females more susceptible to naphthalene-induced injury (van Winkle et al., 2002).

\subsubsection{Air-liquid interface cultures - Model for proximal airway regeneration}

ALI cultures represent an in vitro method for studying mucociliary differentiation, a process required during airway regeneration. For the establishment of ALI cultures, mouse tracheal epithelial cells (MTECs) or human airway epithelial cells are isolated and cultured under submerged conditions on permeable membranes of specialized cell culture inserts (Figure 11). Under submerged conditions, the main population that survives and expands are the basal cells. Once the basal cells reach full confluence, the apical medium is removed to create an ALI that initiates mucociliary differentiation. After approximately 14 (murine) or 21 (human) days, mucociliary differentiation is completed yielding a pseudostratified epithelium. The pseudostratified epithelium contains mostly ciliated and secretory cells, which closely mimics the in vivo situation (You et al., 2002; Ross et al., 2007).

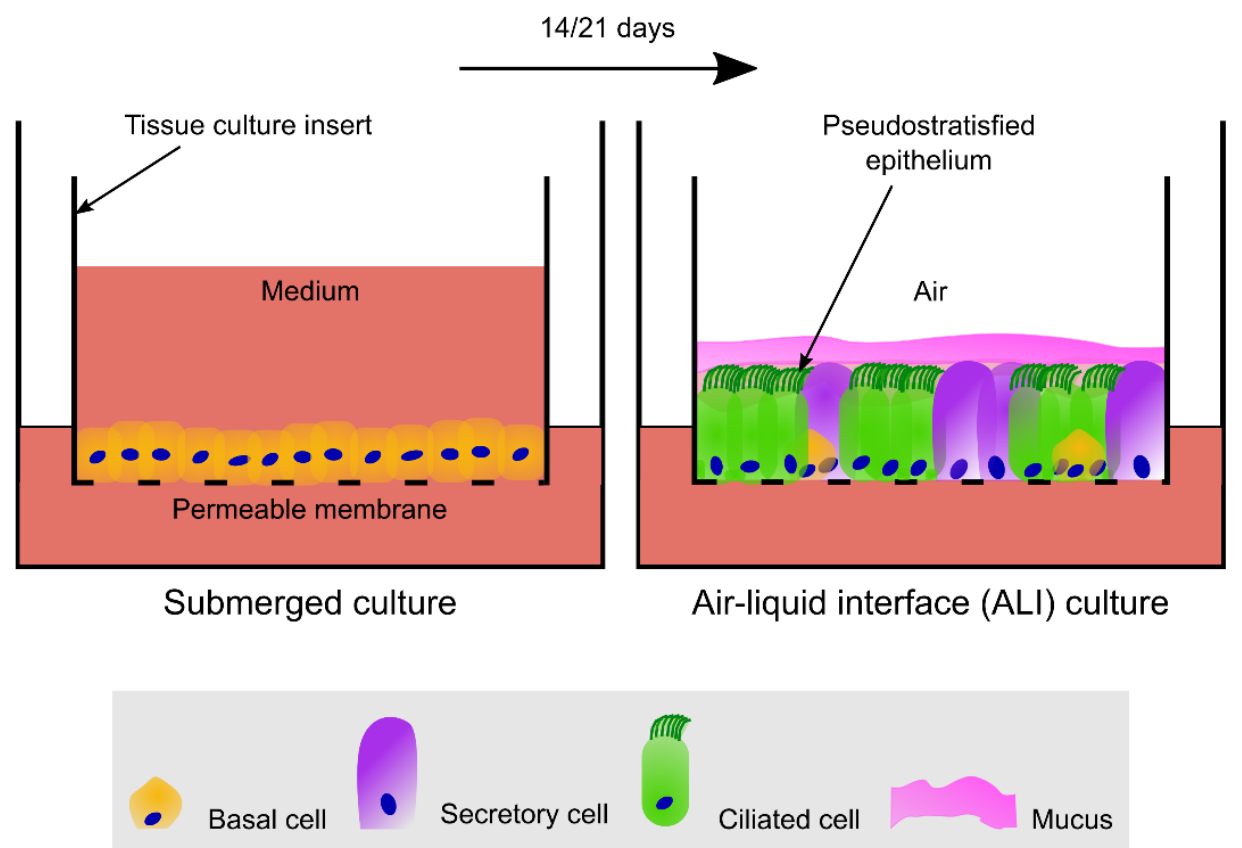

Figure 11: Schematic illustration of ALI cultures.

Isolated human/murine basal cells are grown on a permeable membrane under submerged conditions. Upon confluency, the apical medium is removed to establish an ALI, which allows basal cells to differentiate into a pseudostratified epithelium within 14 (mouse)/21(human) days. Adapted from (Tam et al., 2011). 


\subsection{Preliminary results - TAp73 is a central transcriptional regulator of airway multiciliogenesis}

Alice Nemajerova ${ }^{1}$, Daniela Kramer, ${ }^{2,10}$, Saul S. Siller ${ }^{3,10}$, Christian Herr ${ }^{4,10}$, Orr Shomroni ${ }^{5}$, Tonatiuh Pena ${ }^{5}$, Cristina Gallinas Suazo ${ }^{2}$, Katharina Glaser ${ }^{2}$, Merit Wildung ${ }^{2}$, Henrik Steffen $^{2}$, Anusha Sriraman², Fabian Oberle ${ }^{2}$, Magdalena Wienken², Magali Hennion ${ }^{5}$, Ramon Vidal ${ }^{5}$, Bettina Royen ${ }^{6}$, Mihai Alevra ${ }^{6}$, Detlev Schild ${ }^{6}$, Robert Bals ${ }^{4}$, Jürgen Dönitz ${ }^{7}$, Dietmar Riedel ${ }^{8}$, Stefan Bonn ${ }^{5}$, Ken-Ichi Takemaru ${ }^{3}$, Ute M. Moll ${ }^{1,2}$, and Muriel Lizée,

1) Department of Pathology, Stony Brook University, Stony Brook, New York 11794, USA;

2) Institute of Molecular Oncology, Göttingen University, 37077 Göttingen, Germany;

3) Department of Pharmacology, Stony Brook University, Stony Brook, New York 11794, USA;

4) Department of Internal Medicine V, Saarland University, Homburg 66421, Germany;

5) Computational Systems Biology, German Center for Neurodegenerative Diseases, 37077 Göttingen, Germany;

6) Department of Neurophysiology and Cellular Biophysics, Göttingen University, 37073 Göttingen, Germany;

7) Department of Evolutionary Developmental Genetics, Göttingen University, 37077 Göttingen, Germany;

8) Electron Microscopy, Max-Planck-Institute for Biophysical Chemistry, 37077 Göttingen, Germany;

9) Clinic for Cardiology and Pneumology, Department of Pneumology, University Medical Center Göttingen, 37099 Göttingen, Germany.

adapted from Genes Dev. 2016 Jun 1;30(11):1300-12

http://www.genesdev.org/cgi/doi/10.1101/gad.279836.116

Contribution to the results presented in this section:

Experiments and data analysis for Figure 12 in this thesis were conducted as indicated below:

M. Wildung: Figure 12a-c, h, i, and j

C. Herr: Figure $12 d, e$, and $f$

H. Steffen: Figure $12 \mathrm{~g}$

M. Alevra: Figure 12i and j (data analysis) 


\section{Results}

MCCs play a key role in several processes including mucociliary clearance and fertility, hence defective MCCs are involved in the development of several human diseases such as PCD. However, the transcriptional regulation of multiciliogenesis is poorly understood. To get insights into the pathogenesis of ciliopathies, it is of great importance to elucidate the regulation of multiciliogenesis.

p73 and TAp73 KO mice develop typical characteristics of PCD (hydrocephalus, rhinitis/otitis, and infertility) (Yang et al., 2000; Tomasini et al., 2008; Tomasini et al., 2009; Holembowski et al., 2014; Inoue et al., 2014). Thus, we hypothesized that TAp73 is a novel transcriptional regulator of multiciliogenesis. We specifically aimed to study the role of TAp73 in airways multiciliogenesis in order to explain the chronic airway inflammation observed in $p 73 \mathrm{KO}$ mice.

First, we studied whether isoform-specific TAp73 KO mice (Figure 12a) possess a reduced lung function as an indicator for chronic airway inflammation. Indeed, TAp73 KO mice developed an impaired pulmonary function accompanied with an increased macrophage infiltration, and emphysema (Figure $12 b-f$ ). The impaired lung function along with the chronic inflammation is caused by a reduction in airway ciliation, as indicated by reduced levels of the cilia markers Ac- $\alpha-T U B$ and dynein axonemal light intermediate chain 1 (DNALI1) (Figure $12 \mathrm{~g}$ and $\mathrm{h}$ ). The functional consequence of the reduced ciliation is a marked decline in mucociliary clearance in p73 and TAp73 KO mice compared to wild type (WT) mice (Figure 12i and j, for data on TAp73 KO see online publication (Nemajerova et al., 2016)]). 
a

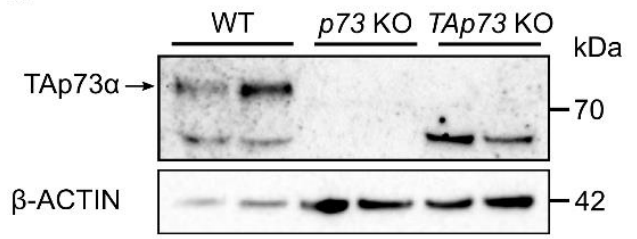

C

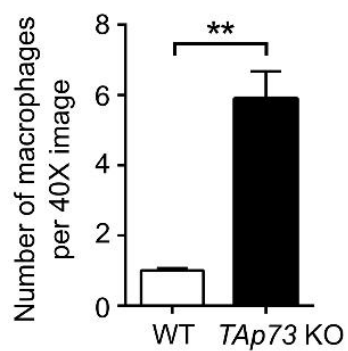

g

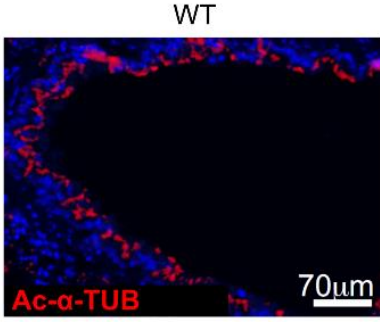

d

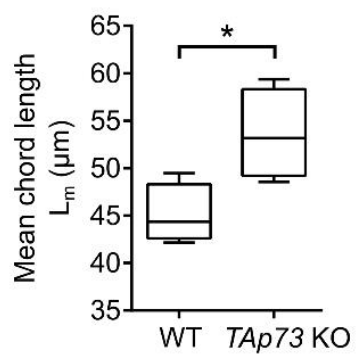

TAp73 KO

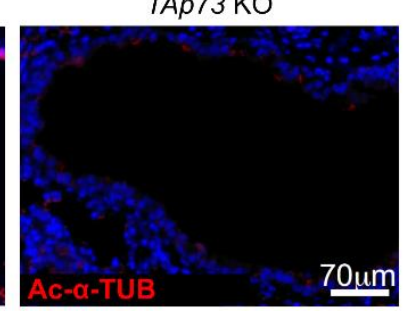

i

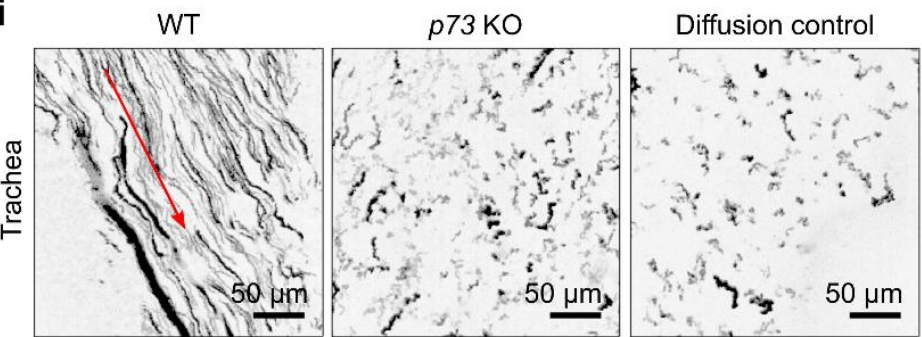

b

e
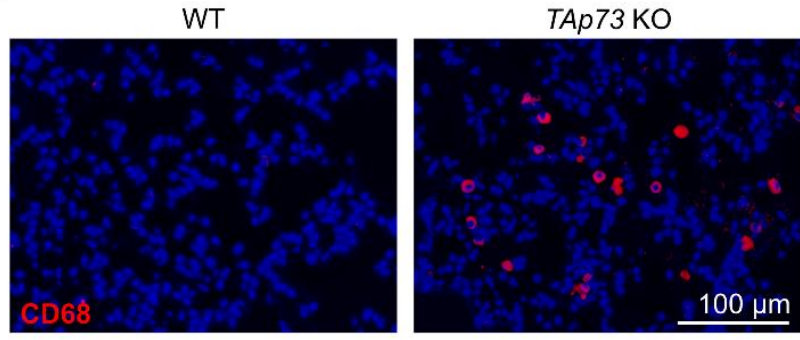

$\mathbf{f}$
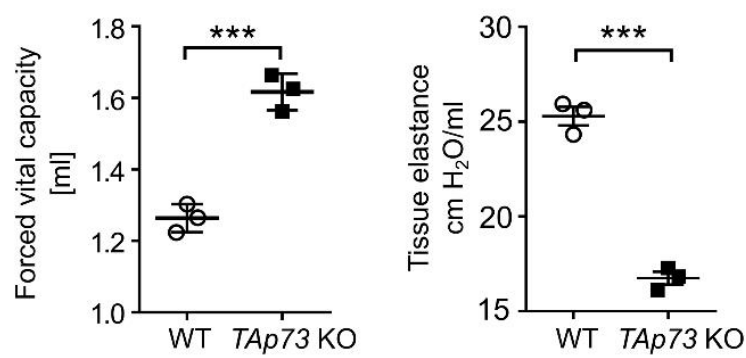

h

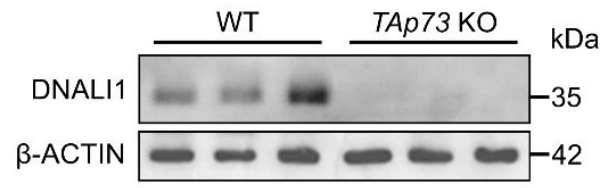

j

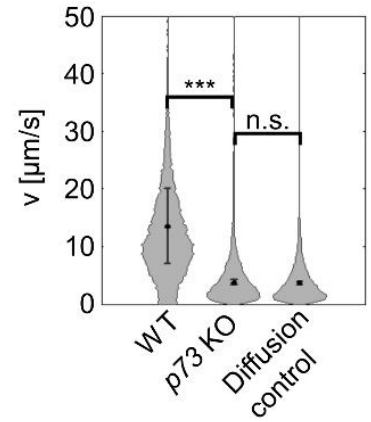

Figure 12: TAp73 deficiency impairs pulmonary function due to a reduced airway ciliation and mucociliary clearance.

a) Immunoblot of total lung lysates from WT, p73, and TAp73 KO mice stained for TAp73 and $\beta$-ACTIN as loading control. b) Macrophage infiltration in TAp73 KO lungs stained with CD68 (red) and 4', 6-Diamidin-2phenylindol (DAPI, blue). c) Quantification of CD68 immunostaining, $n=4$ mice per genotype. d) Quantification of mean chord length as indicator for emphysema in WT and TAp73 KO mice, $n=4$ per genotype. e and f) Assessment of pulmonary function by measuring forced vital capacity (e) and tissue elastance (f) in WT and TAp73 KO mice by spirometry. g) Immunostaining for the axonemal cilia marker Ac$\mathrm{a}-T U B$ (red) on lung sections from WT and TAp73 mice. Nuclei were counterstained with DAPI (blue). h) Immunoblot of total lung lysates from WT and TAp73 KO mice stained for DNALI1, a marker for the IDA of motile cilia. i and j) Fluorescence beads were applied to tracheal explants and tracked by high-speed confocal microscopy. i) Bead trajectories aggregated from 2,000 images over 32 seconds of movie. Red arrow indicates direction of bead transport. j) Bead transport velocity over WT and $p 73 \mathrm{KO}$ trachea. Passive diffusion over dead trachea of both genotypes serves as control. $n=4$ mice per genotype. 18,186, 22,838, and 14,970 tracks for WT, p73 KO, and diffusion control, respectively. 
To identify the mechanism by which TAp73 influences multiciliogenesis, we used genomic analyses and subsequently validated potential TAp73 targets by targeted chromatin immunoprecipitation (ChIP) analysis and reporter-based assays. These analyses established TAp73 as a central regulator of airway multiciliogenesis, since TAp73 directly activated key players of multiciliogenesis such as Foxj1, Rfx2, Rfx3, Myb, miR34b,c, and structural and functional ciliary genes (Figure 13). Thus, TAp73 is necessary for proper BB docking, axoneme extension, and cilia motility in the respiratory system.

Whether TAp73 also contributes to the regulation of multiciliogenesis in other multiciliated organs such as FTs, EDs, and brain ventricles remains unknown. However, a quick look into the phenotype of TAp73 KO mice provides some clues: 1) Female and male TAp73 KO mice are infertile (Tomasini et al., 2008). 2) In females, the oocytes of TAp73 KO mice do not reach the FT, as they are trapped in the bursa (Tomasini et al., 2008; Tomasini et al., 2009). 3) TAp73 KO mice do not develop a hydrocephalus (Tomasini et al., 2008). Proper gamete and zygote transport are achieved by beating of multiple motile cilia and thus, required for fertility (Hess, 2002; Ezzati et al., 2014; Hess, 2015). Hence, it is likely that TAp73 is also necessary for multiciliogenesis in the reproductive systems. Since hydrocephalus, which results from dysfunction of ependymal and CP cilia (Ibanez-Tallon, 2004; Banizs et al., 2005; Banizs et al., 2007), is not observed in TAp73 KO mice, TAp73 might not be a major contributor to brain multiciliogenesis. Due to these indications, we investigated the role of TAp73 on multiciliogenesis in the reproductive tracts and the ventricles of the brain (see section 4.1). 


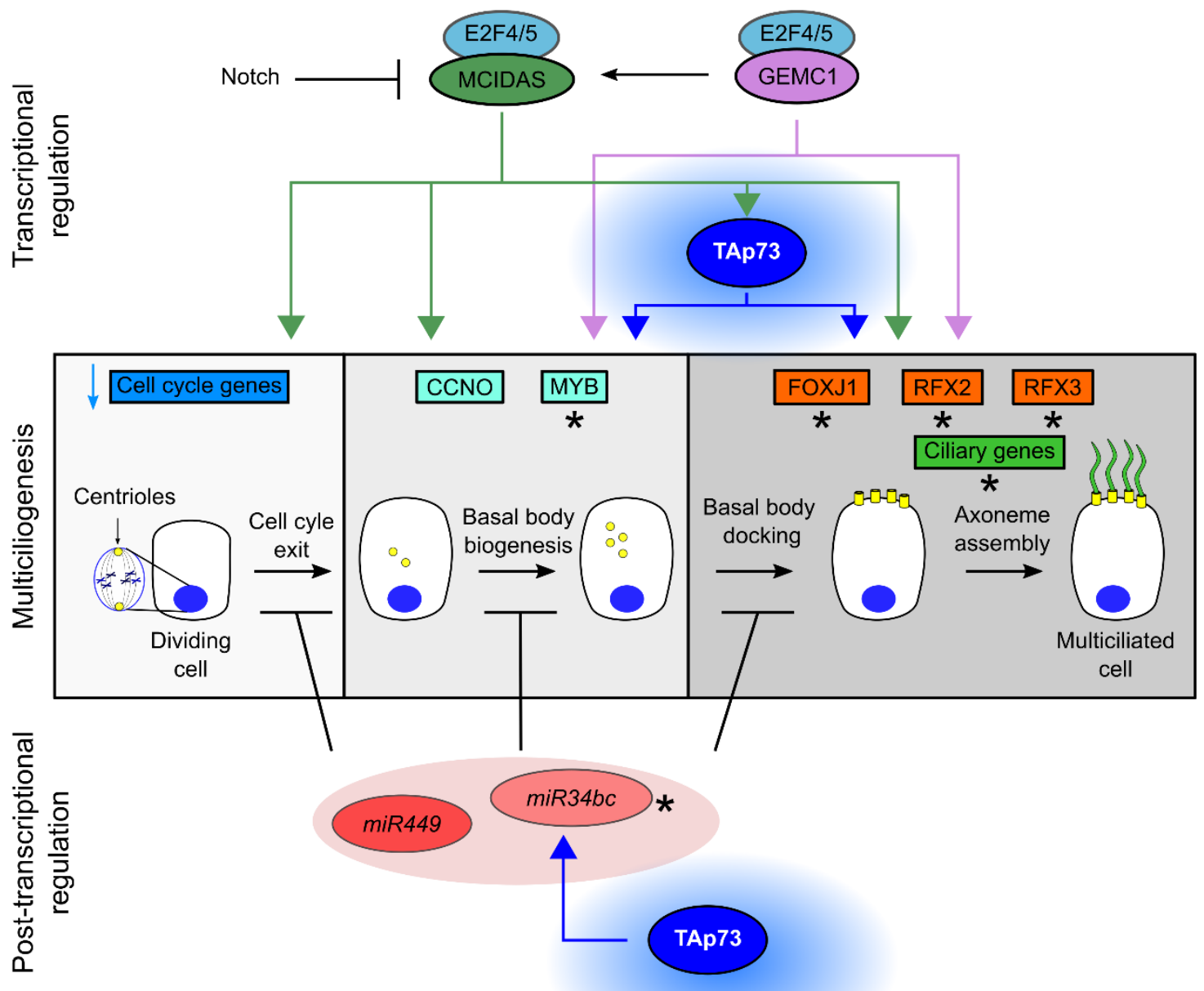

Figure 13: TAp73 is a central regulator of airway multiciliogenesis.

TAp73 acts upstream of known key regulators of multiciliogenesis like Foxj1, Rfx2, Rfx3, Myb, and miR34b,c. In addition, TAp73 directly activates structural and functional ciliary genes. TAp73 targets are indicated with asterisks. Adapted from (Nemajerova et al., 2016). 


\section{OBJECTIVES}

We identified TAp73 as a central regulator of airway multiciliogenesis as it directly activates the expression of several pro-ciliogenic factors (described in section 1.6) (Nemajerova et al., 2016). The first aim of this thesis is to examine whether TAp73 also regulates multiciliogenesis in other multiciliated tissues, including the female and male reproductive tracts as well as the ventricles of the brain.

Multiciliogenesis is not only regulated at the transcriptional level but also at the posttranscriptional level by miRNAs of the miR-34/449 family. Depletion of all family members results in a severe impairment of mucociliary clearance, leading to early lethality (Song et al., 2014, Otto et al., 2017). However, previous reports showed that the expression of miR449 is greatly increased in comparison to miR34 during mucociliary differentiation, highlighting the importance of miR449 (Lizé et al., 2010, Marcet et al., 2011). This brings me to the second aim of this thesis, which is to elucidate the specific function of miR449 alone in mucociliary regeneration and lung homeostasis.

In summary, the overall aim of this thesis is to examine the role of the transcription factor and tumor suppressor TAp73 and the post-transcriptional regulator miR449 in multiciliogenesis to understand the underlying molecular pathology of diseases associated with cilia dysfunction such as PCD and COPD. To achieve that, the specific objectives are the following:

1. Determination of the role of TAp73 in diverse multiciliated tissues. To achieve this purpose, we analyzed TAp73 KO mice focusing on the following aspects:

- Analysis of the expression pattern of TAp73 in different multiciliated tissues.

- Examination of the multiciliated epithelia of the female and male reproductive tracts, and brain ventricles in the absence of TAp73 in mice.

- Identification of pro-ciliogenic target genes of TAp73 in the female and male reproductive system.

- Characterization of the transcriptional regulation of multiciliogenesis in the brains of TAp73 KO mice.

- Analysis of the multiciliated epithelium in the ventricles of TAp73xmiR449 KO mice to support the observed findings in ventricles of TAp73 KO mice.

2. Assessment of the specific function of miR449 in mucociliary regeneration and lung homeostasis. To accomplish this objective, we characterized miR449 KO mice focusing on the following specific points:

- Analysis of expression of miR449 during mucociliary differentiation in vitro and after environmental toxicant exposure in vivo.

- Examination of the airway ciliation under homeostatic and challenged conditions in WT and miR449 KO mice.

- Characterization of the effect of miR449 loss on pulmonary function.

- Identification and validation of a new miR449 target that is required for the maintenance of the multiciliated airway epithelium. 


\section{METHODS}

\subsection{Cell Biology}

\subsubsection{Cell culture, Transfection of human cells}

H1299 cells were cultured in Dulbecco's Modified Eagle Medium (DMEM, Gibco, Thermo Fisher Scientific, Waltham, MA, USA) supplemented with 10\% fetal calf serum (FCS), $2 \mathrm{nM}$ L-glutamine, and $10 \mu \mathrm{g} / \mathrm{ml}$ Ciprofloxacin 500. H1299 cells were reverse-transfected with 12 $\mathrm{nM}$ (RNA analysis) or $20 \mathrm{nM}$ (protein analysis) synthetic pre-miRNAs (Ambion, Thermo Fisher Scientific) of the miR449 family using Lipofectamine 2,000 (Invitrogen, Thermo Fisher Scientific). Scrambled RNA oligonucleotide (negative control (ctrl) \#2) and pre-miR$302^{*}$ were used as controls. Cells were harvested for protein and RNA isolation $48 \mathrm{~h}$ after transfection.

\subsubsection{Analysis of primary cilia assembly and disassembly}

Mouse embryonic fibroblasts (MEFs) were isolated from embryos at E13.5 from pregnant female WT and miR449-/- mice and cultured in DMEM-high glucose (DMEM, Gibco, Thermo Fisher Scientific) supplemented with $10 \%$ heat inactivated FCS, $100 \mathrm{ug} / \mathrm{ml}$ streptomycin, and $100 \mathrm{U} / \mathrm{ml}$ penicillin (Invitrogen, Thermo Fisher Scientific). For the analysis of primary cilia assembly freshly isolated WT and miR449 $/$ MEFs were plated on fibronectin (Merck, Darmstadt, Germany) coated coverslips and exposed to serum-free medium (DMEM supplemented only with $100 \mathrm{ug} / \mathrm{ml}$ streptomycin and $100 \mathrm{U} / \mathrm{ml}$ penicillin) for $60 \mathrm{~h}$ to induce primary cilia assembly. Afterwards, serum was reintroduced for $6 \mathrm{~h}$ to trigger ciliary disassembly. To quantify the percentage of ciliated cells at ciliary assembly and disassembly, MEFs were fixed with $2 \%$ formaldehyde (Merck) at room temperature (RT) for 8 minutes ( $\mathrm{min}$ ) and blocked with 10\% bovine serum allbumin (BSA) (Merck) in phosphatebuffered saline containing $0.1 \%$ Iriton X-100 (PBS-T) for $1 \mathrm{~h} 30$ min at RT. First, MEFs were incubated with primary antibodies diluted in blocking solution overnight at $4^{\circ} \mathrm{C}$ (List of primary antibodies in Table 1) and subsequently stained with secondary antibodies for $1 \mathrm{~h}$ 30 min at RT (List of primary antibodies in Table 2). Nuclei were counterstained with 4', 6Diamidin-2-phenylindol (DAPI). Stainings were visualized using a Leica SP2 laser scanning confocal microscope (Leica Microsystems, Wetzler, Germany). For the quantification of the percentage of ciliated cells only cells, which were positively stained for both Pericentrin and Ac- $\alpha$-TUB were counted as ciliated cells. Experiment was performed by N. Movsisyan. 
Table 1: Primary antibodies.

\begin{tabular}{|c|c|c|c|}
\hline Antibodies & $\begin{array}{l}\text { Dilution } \\
\text { (Application) }\end{array}$ & Company & Catalog [clone] \\
\hline $\begin{array}{l}\text { Mouse monoclonal } \\
\text { anti-Ac- } \alpha-T U B\end{array}$ & $\begin{array}{l}1: 1,000 \text { (IF-P) } \\
1: 4,000 \text { (IF-Fr) }\end{array}$ & Merck & T6793 [6-11B-1] \\
\hline $\begin{array}{l}\text { mouse monoclonal } \\
\text { anti-Ac- } \alpha-T U B\end{array}$ & $1: 250$ (ICC) & $\begin{array}{l}\text { Santa Cruz (Dallas, } \\
T X, U S A)\end{array}$ & sc23950 \\
\hline $\begin{array}{l}\text { Mouse monoclonal } \\
\text { anti-ARL13B }\end{array}$ & $1: 500$ (IF-P) & $\begin{array}{l}\text { UC Davis/NIH } \\
\text { NeuroMab Facility } \\
\text { (Davis, CA, USA) }\end{array}$ & $75-287$ [N295B/66] \\
\hline $\begin{array}{l}\text { Rabbit polyclonal } \\
\text { anti-ARL13B }\end{array}$ & 1:500 (IHC-P) & $\begin{array}{l}\text { Proteintech } \\
\text { (Rosemont, IL, USA) }\end{array}$ & 17711-1-AP \\
\hline $\begin{array}{l}\text { Mouse monoclonal } \\
\text { anti-AQP1 }\end{array}$ & $1: 1,000(\mathrm{IHC}, \mathrm{IF}-\mathrm{P})$ & $\begin{array}{l}\text { Abcam (Cambridge, } \\
\text { UK) }\end{array}$ & ab9566 [1/22] \\
\hline $\begin{array}{l}\text { Rabbit polyclonal } \\
\text { anti-AQP1 }\end{array}$ & $1: 1,000(\mathrm{IF}-\mathrm{P})$ & Merck & AB2219 \\
\hline $\begin{array}{l}\text { Rabbit polyclonal } \\
\text { anti- } \beta \text {-ACTIN }\end{array}$ & $1: 10,000$ (WB) & Abcam & ab8227 \\
\hline $\begin{array}{l}\text { mouse monoclonal } \\
\text { anti-Aurora A }\end{array}$ & $1: 700$ (WB) & Santa Cruz & sc56881 \\
\hline $\begin{array}{l}\text { Rabbit polyclonal } \\
\text { anti-Cytokeratins }\end{array}$ & 1:100 (IHC) & $\begin{array}{l}\text { Agilent } \\
\text { Technologies, Santa } \\
\text { Clara, CA, USA) }\end{array}$ & Z0622 \\
\hline $\begin{array}{l}\text { Goat polyclonal } \\
\text { Anti-CD206 }\end{array}$ & 1:750 (WB) & $\begin{array}{l}\text { Novus Biological } \\
\text { (Littleton, Colorado, } \\
\text { USA) }\end{array}$ & AF2535 \\
\hline $\begin{array}{l}\text { Rabbit polyclonal } \\
\text { anti-DNAl1 }\end{array}$ & $\begin{array}{l}1: 500 \text { (IF-P), } 1: 300 \\
\text { (IF-Fr), 1:700 (WB) }\end{array}$ & Merck & HPA021649 \\
\hline $\begin{array}{l}\text { Goat polyclonal } \\
\text { anti-DNALI1 }\end{array}$ & 1:300 (WB) & Santa Cruz & sc-160296 \\
\hline $\begin{array}{l}\text { Mouse monoclonal } \\
\text { anti-E2F4 }\end{array}$ & 1:300 (WB) & Santa Cruz & sc-6851 [D-3] \\
\hline $\begin{array}{l}\text { Rabbit polyclonal } \\
\text { anti-FOXJ1 }\end{array}$ & $1: 500$ (WB) & Merck & HPA005714 \\
\hline $\begin{array}{l}\text { Mouse monoclonal } \\
\text { anti-HSC70 }\end{array}$ & $1: 20,000$ (WB) & Santa Cruz & sc-7298 [B-6] \\
\hline $\begin{array}{l}\text { Rabbit anti-mouse } \\
\text { IgG H\&L }\end{array}$ & $2 \mu \mathrm{g}(\mathrm{ChIP})$ & Abcam & Ab46540 \\
\hline $\begin{array}{l}\text { Rabbit monoclonal } \\
\text { anti-KI-67 }\end{array}$ & 1:100 (IF-P) & Abcam & ab16667 [SP6] \\
\hline $\begin{array}{l}\text { Rabbit polyclonal } \\
\text { anti-OTX2 }\end{array}$ & 1:500 (IHC-P) & Merck & AB9566 \\
\hline $\begin{array}{l}\text { Rabbit polyclonal } \\
\text { anti-Pericentrin }\end{array}$ & $1: 1,500$ (ICC) & Abcam & ab4448 \\
\hline $\begin{array}{l}\text { Rabbit monoclonal } \\
\text { anti-p21 }\end{array}$ & $1: 300$ (WB) & Abcam & $\begin{array}{l}\text { ab188224 } \\
\text { [EPR18021] }\end{array}$ \\
\hline $\begin{array}{l}\text { Rabbit monoclonal } \\
\text { anti-P73 }\end{array}$ & $\begin{array}{l}\text { 1:100 (IF-P, IHC-P), } \\
1: 300 \text { (WB) }\end{array}$ & Abcam & ab40658 [EP436Y] \\
\hline
\end{tabular}




\begin{tabular}{|c|c|c|c|}
\hline Antibodies & $\begin{array}{l}\text { Dilution } \\
\text { (Application) }\end{array}$ & Company & Catalog [clone] \\
\hline $\begin{array}{l}\text { Rabbit polyclonal } \\
\text { anti-P73 }\end{array}$ & $2 \mu \mathrm{g}(\mathrm{ChIP})$ & Abcam & Ab14430] \\
\hline $\begin{array}{l}\text { Mouse monoclonal } \\
\text { anti-TIMP1 }\end{array}$ & $1: 500$ & $\begin{array}{l}\text { Invitrogen, Thermo } \\
\text { Fisher Scientific }\end{array}$ & MA1-773 \\
\hline $\begin{array}{l}\text { Rabbit polyclonal } \\
\text { anti-TTR }\end{array}$ & 1:100 (IHC-P) & Proteintech & 1189-1-AP \\
\hline $\begin{array}{l}\text { Rabbit polyclonal } \\
\text { anti-y-Tubulin }\end{array}$ & 1:400 (WB) & Merck & T5192 \\
\hline
\end{tabular}

Table 2: Secondary antibodies.

\begin{tabular}{|c|c|c|c|}
\hline Antibodies & Dilution (Application) & Company & Catalog \# \\
\hline Alexa Fluor 488 donkey anti-mouse & $1: 500$ (IF-P) & $\begin{array}{l}\text { Invitrogen, } \\
\text { Thermo Fisher } \\
\text { Scientific }\end{array}$ & A21202 \\
\hline Alexa Fluor 488 goat anti-mouse & $1: 1,000($ ICC) & $\begin{array}{l}\text { Invitrogen, } \\
\text { Thermo Fisher } \\
\text { Scientific }\end{array}$ & A-11001 \\
\hline $\begin{array}{l}\text { Alexa Fluor } 488 \text { chicken anti- } \\
\text { mouse }\end{array}$ & $1: 200$ (IF-Fr) & $\begin{array}{l}\text { Invitrogen, } \\
\text { Thermo Fisher } \\
\text { Scientific }\end{array}$ & A-21200 \\
\hline Alexa Fluor 594 goat anti-rabbit & $1: 500$ (IF-P) & $\begin{array}{l}\text { Invitrogen, } \\
\text { Thermo Fisher } \\
\text { Scientific }\end{array}$ & A11012 \\
\hline Alexa Fluor 633 goat anti-rabbit & $1: 1,000($ ICC) & $\begin{array}{l}\text { Invitrogen, } \\
\text { Thermo Fisher } \\
\text { Scientific }\end{array}$ & A-21071 \\
\hline $\begin{array}{l}\text { Rhodamine Red-X } \mathrm{XM}^{\mathrm{TM}} \text { donkey } \\
\text { anti-rabbit }\end{array}$ & $1: 200$ (IF-Fr) & $\begin{array}{l}\text { Jackson } \\
\text { Immunoresearch } \\
\text { (West Grove, PA, } \\
\text { USA) }\end{array}$ & 711-295-152 \\
\hline $\begin{array}{l}\text { Peroxidase-conjugated donkey } \\
\text { anti-mouse }\end{array}$ & $1: 10,000$ (WB) & $\begin{array}{l}\text { Jackson } \\
\text { Immunoresearch }\end{array}$ & $715-036-150$ \\
\hline $\begin{array}{l}\text { Peroxidase-conjugated donkey } \\
\text { anti-goat }\end{array}$ & $1: 10,000$ (WB) & $\begin{array}{l}\text { Jackson } \\
\text { Immunoresearch }\end{array}$ & 705-036-147 \\
\hline $\begin{array}{l}\text { Peroxidase-conjugated donkey } \\
\text { anti-rabbit }\end{array}$ & $1: 10,000$ (WB) & $\begin{array}{l}\text { Jackson } \\
\text { Immunoresearch }\end{array}$ & 711-036-152 \\
\hline $\begin{array}{l}\text { Biotin-SP-conjugated AffiniPure } \\
\text { Goat Anti-Rabbit IgG }\end{array}$ & 1:1,000 (IHC-P) & $\begin{array}{l}\text { Jackson } \\
\text { ImmunoResearch }\end{array}$ & $111-065-144$ \\
\hline $\begin{array}{l}\text { Biotin-SP-conjugated AffiniPure } \\
\text { Donkey Anti-Mouse }\end{array}$ & $1: 1,000(\mathrm{IHC}-\mathrm{P})$ & $\begin{array}{l}\text { Jackson } \\
\text { ImmunoResearch }\end{array}$ & 715-065-151 \\
\hline
\end{tabular}




\subsubsection{Air-liquid interface cultures}

MTECs were isolated and cultivated using ALI conditions as described previously (You et al., 2002). Note, minor changes regarding the culture and differentiation medium were applied as highlighted below. Briefly, tracheae from WT and miR449/- mice (10-16 weeks old) were excised and after pronase digestion (Merck), epithelial cells were seeded onto collagen coated transwell culture inserts $(12 \mathrm{~mm}$ diameter, $0.4 \mu \mathrm{m}$ pores, polyester, Corning, New York, USA). Cells were cultured under submerged conditions in airway epithelial cell growth medium supplemented with growths factors (Promocell, Heidelberg, Germany), $100 \mathrm{U} / \mathrm{ml}$ penicillin, and $100 \mu \mathrm{g} / \mathrm{ml}$ streptomycin to allow proliferation. Upon confluence, ALI condition was generated by removing the apical chamber medium and changing the basolateral medium to the differentiation medium consisting of DMEM/F-12 (Gibco) supplemented with $2 \%$ Ultroser G (Pall, Port Washington, New York, USA). At differentiation day (d) 6 , the entire ALI culture membrane was fixed with $4 \%$ paraformaldehyde (PFA) and embedded in paraffin. For one ALI culture, MTECs pooled from three mice were used. Human ALIs were performed in a former study (Lizé et al., 2010a) and only RNA was recovered to complete the presented work.

\subsubsection{Luciferase assay}

Luciferase assay was performed as previously described (Nemajerova et al., 2016). Briefly, Saos2 cells were transfected with pcDNA3.1 empty vector, or pcDNA3.1 vector carrying $P 73$ isoforms, E2F4, or MCIDAS. Moreover, a firefly luciferase reporter construct containing the putative E2F-binding sequences of miR449 genomic region (WT) or the P73-binding sequence of MLCK genomic region (WT) were transfected (Table 3). In addition, the same sequences lacking the predicted binding motifs (mutant) were transfected (Table 3). Moreover, a Renilla TK luciferase vector was co-transfected. At $24 \mathrm{~h}$ after transfection, cells were harvested, and the luciferase activities were measured using the dual luciferase assay. Firefly luciferase activities were determined relative to those of the Renilla TK luciferase vector and normalized to the mean value of samples from the control vector. As described in (Wildung et al., 2018). E2F4/Cdc20b luciferase assay was performed by $M$. Lizé. 
Table 3: $\quad$ Luciferase constructs.

\begin{tabular}{|c|c|c|c|c|}
\hline Construct name & DNA sequence of inserts & Vector & $5^{\prime}$ RS & 3' RS \\
\hline $\begin{array}{c}\text { WT } \\
\text { miR449/Cdc20b } \\
\text { WT E2F } \\
\text { binding site }\end{array}$ & $\begin{array}{l}\text { GCCAGAAAGCTGAGCACACTGGGGA } \\
\text { CTCCGTGATAAAGGGGGAGAGGAAG } \\
\text { ATATTGAGGGTTGAGGAAGAGGTCTG } \\
\text { GCGGGAAATGACAGGGAACCAGATG } \\
\text { GGCTGTGCAGCCTTAGCTGCCCATCT } \\
\text { GAGCTGCCAAGAGAGCCGAGTTGTG } \\
\text { CCATATGGCAGGAG }\end{array}$ & pGL4.23 & Nhel & EcoRV \\
\hline $\begin{array}{c}\text { Mut } \\
\text { miR449/Cdc20b } \\
\text { Mut E2F } \\
\text { binding site }\end{array}$ & $\begin{array}{l}\text { GCCAGAAAGCTGAGCACACTGGGGA } \\
\text { CTCCGTGATAAAGGGGGAGAGGAAG } \\
\text { ATATTGAGGGTTGAGGAAGAGGTCTG } \\
\text { GACAGGGAACCAGATGGGCTGTGCA } \\
\text { GCCTTAGCTGCCCATCTGAGCTGCCA } \\
\text { AGAGAGCCGAGTTGTGCCATATGGCA } \\
\text { GGAG }\end{array}$ & pGL4.23 & Nhel & EcoRV \\
\hline $\begin{array}{c}\text { WT MLCK } \\
\text { WT p73 } \\
\text { binding site }\end{array}$ & $\begin{array}{l}\text { CACAGGGAACCTGTAATCCCAGCACT } \\
\text { TTGGGAGGCCAAGGCAAGTCGACCA } \\
\text { CTTGAGCCCAGGAGGTCGAGACAAG } \\
\text { CCTGGACAACACGGCAAACCACAAC } \\
\text { TCTACAAAAAAACACAAAAATTAACTG } \\
\text { GGAATGGTGGTGCATGCCTGTGGTCT } \\
\text { CAGCCACTCAAGGGGCTGAGGCAG }\end{array}$ & pGL4.23 & Nhel & HindIII \\
\hline $\begin{array}{c}\text { Mut MYLK } \\
\text { Mut p73 } \\
\text { binding site }\end{array}$ & $\begin{array}{l}\text { CACAGGGAACCTGTAATCCCAGCACT } \\
\text { TTGGGAGGCCAAGGCAAGTCGACCA } \\
\text { CTTGAGCTCGAGAGAACGGCAAACCA } \\
\text { CAACTCTACAAAAAAACACAAAAATTA } \\
\text { ACTGGGTGGTGCCTGTGGTCTCAGC } \\
\text { CACTCAAGGGGCTGAGGCAG }\end{array}$ & pGL4.23 & Nhel & HindIII \\
\hline
\end{tabular}

The strongest E2F binding site is depicted in yellow and the removed sequence in bold underlined. Two consensus sequences with lower score were retained in the mutant (pink and red). RS: Restriction site. p73 binding sites in yellow, bold underlined and green respectively; deleted bases in red)

\subsection{Mouse Work, Mouse Models}

\subsubsection{Mice}

TAp73 mutant mice with targeted deletion of exons 2 and 3 of the $\operatorname{Trp} 73$ gene were a generous gift from Dr. Tak Mak (Princess Margaret Cancer Centre, Toronto, Canada) (Tomasini et al., 2008). miR449 mutants were previously described (Song et al., 2014). Both strains were maintained in C57B//6 background (n8) at the animal facility of the European Neuroscience Institute Göttingen, Germany in full compliance with institutional guidelines. The study was approved by the Animal Care Committee of the University Medical Centre Goettingen and the authorities of Lower-Saxony under the number 16/2069 (TAp73xmiR449 KO mice) and G12/963 (naphthalene). As described in (Wildung et al., 2018). 
WT (C57BL/6) mice used for DEP experiments were obtained from Harlan Laboratories (Horst, The Netherlands) and maintained in the animal facility of the Ghent University. The Animal Ethical Committee of the Faculty of Medicine and Health Sciences of Ghent University approved the in vivo manipulations using DEP.

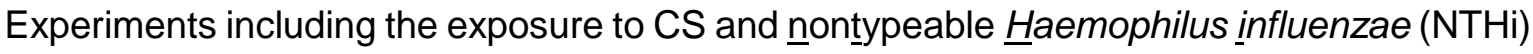
were approved by the "Landesamt für Soziales, Gesundheit und Verbraucherschutz" of the State of Saarland following the national guidelines for animal treatment. During the experiment WT and miR449- mice were maintained under specific pathogen-free conditions in the animal facility of the Institute for Experimental Surgery of the Saarland University Homburg.

\subsubsection{Naphthalene-induced injury model}

Naphthalene (Merck) was dissolved in sunflower oil to a concentration of $30 \mathrm{mg} / \mathrm{ml}$ in a sterile environment on the day of use.12-14 weeks old male WT and miR449-/ mice were injected with naphthalene $(200 \mathrm{mg} / \mathrm{kg}$ ) or with oil (vehicle control) i.p. in the morning on $\mathrm{d} 0$. Changes in body weight were monitored every day during the first week and then again at the endpoint (d14). Lung analysis was performed at d1, d3, d7, and d14.

\subsubsection{DEP-induced acute inflammation model}

DEP instillation was performed as described previously (Provoost et al., 2016). Briefly, DEP (standard reference material (SRM) 2975) was obtained from the National Institute for Standards and Technology (Gaithersburg, MD, USA) and suspended in saline containing $0.05 \%$ Tween 80 to a concentration of $2 \mathrm{mg} / \mathrm{ml}$. 6-8 weeks old anesthetized mice (i.p. ketamine (70 mg/kg; Ketamine 1,000 CEVA, Ceva Sante Animale, Libourne, France) xylazine (7 mg/kg; Rompun 2\%, Bayer, Leverkusen, Germany) were held vertically and 50 $\mu \mathrm{l}$ saline or DEP solution $(100 \mu \mathrm{g})$ was pipetted just above their vocal cords. By grasping the tongue, the mice were prevented from swallowing. Mice were instilled at $d 1, d 4$, and $d 7$ and sacrificed at $d 9$ by a lethal dose of i.p. pentobarbital (Ceva Sante Animale). Experiment was performed by S. Provoost.

\subsubsection{NTHi-induced chronic inflammation model}

A clinical isolate of NTHi was grown on selective chocolate agar supplemented with $300 \mu \mathrm{l}$ IsoVitaleX per $10 \mathrm{~cm}$ plate (Becton Dickinson, Heidelberg, Germany) for $24 \mathrm{~h}$ at $37^{\circ} \mathrm{C}$ in $5 \%$ $\mathrm{CO}_{2}$. After harvesting, bacteria were incubated for $24 \mathrm{~h}$ in brain-heart infusion $(700 \mathrm{ml}$ ) broth (Becton Dickinson) supplemented with $3.5 \mathrm{mg} / \mathrm{ml} \beta$-Nicotinamide adenine dinucleotide (Merck) and 5\% Fildes enrichment (Becton Dickinson). After a centrifugation step at 2,500 g for $15 \mathrm{~min}$ at $4^{\circ} \mathrm{C}$, the culture was washed, resuspended in PBS $(20 \mathrm{ml})$, and heat inactivated at $70^{\circ} \mathrm{C}$ on a shaker for $45 \mathrm{~min}$. Following sonication (three times for 30 seconds), the protein concentration was determined and adjusted to $2.5 \mathrm{mg} / \mathrm{ml}$ using the 
Pierce BCA-protein assay (Thermo Fisher Scientific). For bacterial challenge, 4 months old WT and $m i R 449^{-/}$mice were transferred to a plexiglass box connected to a Pari MASTER ${ }^{\circ}$ nebulizer (Pari GmbH, Starnberg, Germany) and treated with the inactivated NTHi lysate for $1 \mathrm{~h}$ per day and 5 times per week for 3 months. Experiment was performed by $C$. Herr.

\subsubsection{Cigarette smoke-induced COPD model}

4 months old WT and miR449-/ mice were exposed to CS (3R4F cigarettes, College of Agriculture, Reference Cigarette Program, University of Kentucky, Lexington, Kentucky, USA) using a TE-10 smoking machine (Teague Enterprises, Woodland, California, USA). The concentration of CS was $120 \mathrm{mg} / \mathrm{m}^{3}$ total suspended particles. Mice were subjected to $\mathrm{CS}$ for $5 \mathrm{~h}$ per day and 5 days per week over a period of 6 months. The daily smoking protocol consisted of three smoking phases each with $87 \mathrm{~min}$, which were interrupted by room-air exposures for $40 \mathrm{~min}$. Experiment was performed by $C$. Herr.

\subsubsection{Pulmonary function}

Pulmonary function analysis was carried out using the FlexiVent system (Scireq, Montreal, Canada). Mice were anesthetized by a mixture of xylazine (Rompun, $7 \mathrm{mg} / \mathrm{kg}$, Bayer) and ketamine (Ursotamin, $100 \mathrm{mg} / \mathrm{ml}$, Serumwerk Bernburg, Bernburg, Germany). Subsequently, the trachea was exposed and cannulated using the $18 \mathrm{G}$ cannula supplied with the FlexiVent system. Data acquisition was performed with the flexiWare 7.1 software (Scireq, Montreal, Canada). Experiment was performed by C. Herr.

\subsubsection{Bronchoalveolar lavage}

Prior to bronchoalveolar lavage (BAL) the animals were deeply anesthetized with a mixture of ketamine (Ursotamin, $105 \mathrm{mg} / \mathrm{ml}$, Serumwerk Bernburg) and xylazine (Rompun, $7 \mathrm{mg} / \mathrm{kg}$, Bayer). After reaching anesthesia the trachea was exposed and cannulated. The BAL was performed by rinsing the lungs 3 times repeatedly with $1 \mathrm{ml}$ sterile PBS containing protease inhibitors (cOmplete ULTRA Tablets, Mini, Roche Diagnostic, Mannheim, Germany). The collected BAL fluid (BALF) was centrifuged for $5 \mathrm{~min}$ at $300 \mathrm{~g}$. The number of viable cells was determined by resuspending the cell pellet in $1 \mathrm{ml}$ of sterile PBS and counting on an improved Neubauer chamber after trypan blue staining. For the cellular composition of the BALF a cytospin was stained with DiffQuik (Medion Diagnostic, Gräfelfing, Germany) and at least 200 cells were counted and differentiated based on their morphology. Experiment was performed by C. Herr.

\subsubsection{Stereology}

For stereological analysis, the lung was prepared for uniform random sampling as described previously (Knust et al., 2009). Briefly, fixation of the lung was performed by instillation of freshly prepared PBS-buffered $4 \%$ formalin under a constant hydrostatic pressure of $20 \mathrm{~cm}$ 
for $20 \mathrm{~min}$. After that, the trachea was ligated to preserve intrapulmonary pressure. For additional fixation, the lung was incubated in PBS-buffered $4 \%$ formalin for $24 \mathrm{~h}$. The lung volume used as reference for stereological measurements was determined by fluid displacement. After embedding in agar, the lung was cut into regular slices prior to paraffin embedding. Stereological analysis was performed on hematoxylin and eosin (H\&E) stained lung sections. The mean chord length (mean linear intercept, $L_{m}$ ) was determined using the Visiopharm Integrator System package (Visiopharm, Hoersholm, Denmark) on an Olympus BX51 microscope equipped with an 8-position slide loader (Olymus, Tokio, Japan). Experiment was performed by C. Herr.

\subsubsection{Tissue sample preparation for protein and RNA analyses}

Mice were euthanized with $\mathrm{CO}_{2}$ followed by cervical dislocation. Afterwards, tissues were isolated, washed in cold PBS, and directly snap-frozen in liquid nitrogen until use. For the analyses of the ventricles of the brain coronal slices with an enrichment of the lateral ventricle were used, as indicated by the blue dotted lines in Figure 14e. EDs were isolated by cutting the EDs at connection to the testis on the one end and to the caput epididymis at the other end. Blue dotted lines in Figure 14a indicate the ED cutting sites. FTs were cut at the distal and proximal part to remove the ovary and uterus, respectively (Figure 14c). For the analysis of the airways, the lobus cranialis and the lobus caudalis were used for protein and RNA, respectively. The entire trachea until the bifurcation was used for the analyses.

\subsection{Human samples}

\subsubsection{Human samples of the male reproductive duct}

Human epididymis samples were procured with informed consent from two patients (42 and 41 years of age, respectively). All experimental procedures were approved and performed in accordance with the requirements set forth by Ethics Committee of the University Medical Centre Goettingen (application number: 18/2/16). As described in (Wildung et al., 2018).

\subsubsection{Human COPD bronchial biopsy samples, mRNA and miRNA profiling, and correlation studies}

Bronchial biopsy specimens were collected from 57 COPD patients who were part of the Groningen Leiden Universities and orticosteroids in Obstructive LLung Disease (GLUCOLD) study (Lapperre et al., 2006). In the current study, only the patients at baseline were included. All patients were stable, either current or ex-smokers, and not on corticosteroid therapy. They had irreversible airflow limitation (post-bronchodilator forced expiratory volume in $\underline{1}$ second (FEV 1 ) and FEV 1 /inspiratory vital capacity (IVC) $<90 \%$ confidence interval of the predicted value) and chronic respiratory symptoms. The local 
medical ethics committee approved the study and all patients gave their written informed consent.

mRNA and miRNA profiling were performed at Boston University Microarray Resource Facility using GeneChip ${ }^{\circledR}$ Whole Transcript Sense Target Labeling Assay (Affymetrix, Santa Clara, CA, USA) and FlashTag ${ }^{\mathrm{TM}}$ Biotin HSR Labeling Kit (Affymetrix), respectively. Detailed methods on the profiling and processing of microarray data were described previously (Tasena et al., 2018). Using the matched mRNA and miRNA profiles of the same patient, Pearson correlation coefficient was used to identify genome-wide mRNAs of which expression was correlated with miR-34/449 expression. Benjamini-Hochberg procedure was applied for multiple test correction. Significant correlation was defined using false discovery rate adjusted $p$-value false discovery rate $(F D R)<0.05$.

To assess the role of miR449a in cilia development and function, the list of mRNAs positively correlated with miR449a was compared to the previously reported list of ciliaassociated genes (Nemajerova et al., 2016). Moreover, gene-set enrichment analysis (GSEA, v3.0) (Subramanian et al., 2005) was performed to identify biological processes (Gene Ontology, v6.1) in which miR-34/449 positively correlated genes plays a role. Genome-wide mRNAs were ranked based on their t-statistics representing the strength of their correlation with miR449a expression. A p-value was calculated after 1,000 permutations were performed. Described and performed by $\mathrm{H}$. Tasena.

\subsection{Biochemistry}

\subsubsection{Western blot}

Samples were homogenized in RIPA buffer (20 mM TrisHCl pH 7.5, $150 \mathrm{mM} \mathrm{NaCl}, 9.5 \mathrm{mM}$ EDTA, $1 \%$ Triton X100, $0.1 \%$ SDS, $1 \%$ sodium deoxycholate) supplemented with urea (2.7 M) and protease inhibitors (Complete Mini EDTA-free, Roche). Equal amounts of protein extracts were separated by SDS-polyacrylamide gels prior to transfer onto a nitrocellulose membrane and incubated with primary antibodies (List of antibodies in Table 1). The membrane was washed and incubated for $1 \mathrm{~h}$ with horse radish peroxidase (HRP)conjugated secondary antibodies (List of antibodies in Table 2) followed by chemiluminescence detection. $\beta$-ACTIN or heat shock cognate $\underline{70} \mathrm{kDa}$ protein (HSC70) were used as protein loading controls. As described in (Wildung et al., 2018).

\subsubsection{Histology and Immunostaining}

For histology, PFA-fixed, paraffin-embedded 3- $\mu \mathrm{m}$ tissue sections were stained with H\&E. For immunostaining, PFA-fixed, paraffin-embedded tissue sections (mouse and human tissues: $3 \mu \mathrm{m}$, ALI membranes: $7 \mu \mathrm{m}$ ) were dehydrated and treated with heat-induced epitope retrieval (sodium citrate, $\mathrm{pH}$ 6.0). Cryosections were directly fixed with $4 \%$ PFA for $10 \mathrm{~min}$ at RT. For immunohistochemistry $(\mathrm{IHC})$, endogenous peroxidase activity was 
quenched with $3 \% \mathrm{H}_{2} \mathrm{O}_{2}$ for 10 min after antigen retrieval. Subsequently tissue sections were blocked with 10\% FCS in PBS-T (for cryosections: 1\% BSA in PBS-T) and incubated with primary antibodies (List of antibodies in Table 1).

For IHC, biotinylated secondary antibodies were applied for $1 \mathrm{~h}$ at RT (List of antibodies in Table 2), after which avidin enzyme complex and substrate/chromogen were used for color development (Vector laboratories, Burlingame, CA, USA). Stained tissue sections were counterstained with hematoxylin.

For IF, sections were stained with fluorescently labelled secondary antibodies (List of antibodies in Table 2) for $1 \mathrm{~h}$ at RT. Nuclei were counterstained with DAPI.

Images were acquired on a ZEISS AxioScope A1 microscope (ZEISS, Oberkochen, Germany), except tracheae images, which were viewed on a ZEISS confocal LSM 510 and 880 microscope. As described in (Wildung et al., 2018).

\subsubsection{Quantification of cilia markers}

The expression of cilia markers in immunofluorescence (IF) images (converted to 8-bit) was quantified using the ImageJ software (Schindelin et al., 2012). Briefly, after selecting the region of interest, unspecific signals were removed, and a threshold was set to identify the positive signal. To measure the area of the positive cilia staining the Analyze Particle tool was applied. Values of the cilia area were normalized to the length of the respiratory epithelium measured. As described in (Wildung et al., 2018). For length measurements of motile cilia (ALI cultures, CP) the line tool from the ImageJ software was used. Cilia length was defined by the area positive for both cilia markers used.

\subsection{Molecular Biology}

\subsubsection{RNA extraction, quantitative PCR}

Total RNA from murine tissues was isolated by Extrazol (7BioScience, Hartheim, Germany)/Chloroform extraction followed by $80 \%$ ethanol precipitation at $-20^{\circ} \mathrm{C}$. The miRVana kit (Invitrogen, Thermo Fisher Scientific) was used for RNA isolation from human ALI cultures. For cDNA synthesis, $1 \mu \mathrm{g}$ of RNA was incubated with the M-MuLV reverse transcriptase and a mix of random nonameric and polyA tail primers at $42^{\circ} \mathrm{C}$ for $1 \mathrm{~h}$ in a total volume of $50 \mu \mathrm{l}$. For reverse transcription quantitative polymerase chain reaction (RTqPCR) all reactions were set up in triplicate with self-made SYBR Green qPCR Mix (Tris$\mathrm{HCl}[75 \mathrm{mM}],\left(\mathrm{NH}_{4}\right) 2 \mathrm{SO}_{4}[20 \mathrm{mM}]$, Tween-20 [0.01\% v/v], $\mathrm{MgCl}_{2}$ [3 mM], Triton X-100 [0.25\% v/v], SYBR Green I [1:40,000], dNTPs [0.2 mM] and Taq-polymerase [20 U/ml]) using $250 \mathrm{nM}$ of each gene-specific primers (List of primers in Table 4). Standard curve or $\Delta \triangle \mathrm{Ct}$ method were used to assess relative transcript content. Transcript of interests were normalized to the reference transcript of ribosomal phosphoprotein P0 ( $R p / p 0$, or $36 b 4)$ and normalized to the mean value of control samples. The results for each sample were 
obtained by averaging transcript levels of technical triplicates. No RT controls and dilution curves as well as melting curves and gel electrophoresis assessment of amplicons were performed for all primer combinations. As described in (Wildung et al., 2018).

Copy number in RNA samples was determined by qPCR using a murine TAp73 plasmid (MC219984, Origene, Rockville, USA) with a known copy number as standard curve. Copy number of the TAp73 plasmid was determined using the following formula:

number of copies $=\left(\right.$ plasmid amount $\left.[\mathrm{ng}]{ }^{*} 6.022 \times 10^{23}[\mathrm{molecules} / \mathrm{mole}]\right) /($ plasmid length $[\mathrm{bp}]{ }^{*} 1 \times 10^{9}[\mathrm{ng} / \mathrm{g}]{ }^{*} 650[\mathrm{~g} / \mathrm{mol} \mathrm{l})$. As described in (Wildung et al., 2018).

For miRNA quantification, TaqMan MicroRNA Assays (Applied Biosystems, Thermo Fisher Scientific) for murine and human members of the miR-34/449 family were performed according to the manufacturer's instructions with U6 small nuclear $\underline{R N A}$ (snRNA) as reference gene. As described in (Wildung et al., 2018).

\subsubsection{Small RNA sequencing}

The libraries for small RNA samples were prepared using TruSeq Small RNA Library Prep Kit -Set A (24 rxns, Set A: indexes 1-12; Cat No: RS-200-001, Illumina, San Diego, CA, USA) using $1 \mu \mathrm{g}$ of total RNA according to manufacturer's recommendations. Samples were sequenced on the Illumina HiSeq 4,000 using a 50 bp single-end approach. Mapping, prediction of novel miRNAs, quality control, and differential expression (DE) analysis were carried out using Oasis2.0 (Oasis: online analysis of small RNA deep sequencing data) (Capece et al., 2015). In brief, FASTQ files were trimmed with cutadapt 1.7.1 (Martin, 2011) removing Truseq adapter sequences (TGGAATTCTCGGGTGCCAAGG) followed by removing sequences smaller than 15 or larger than 32 nucleotides. Trimmed FASTQ sequences were aligned to mouse sRNAs using STAR version 2.4.1d (Dobin et al., 2013) with a mismatch of $5 \%$ of the sequence length and by utilizing the following databases: Mirbase version 21 for miRNAs, piRNAbank V.2 for piwiRNA, and Ensembl v84 for small nuclear RNA (snRNA), small nucleolar RNA, and ribosomal RNA. Counts per small RNA were calculated using featureCounts v1.4.6 (Liao et al., 2014). Novel miRNAs were searched for using miRDeep2 version 2.0.0.5 (Friedländer et al., 2012). DE of small RNA was determined by DESeq2 (Love et al., 2014), where small RNAs were considered differentially expressed with an adjusted $p$-value $<0.05$ and absolute log2 fold-change $>1$. The small RNA-sequencing (RNA-seq) data sets were submitted to Gene Expression Omnibus (GEO), accession number GSE108385. As described in (Wildung et al., 2018). Experiment was performed by $O$. Shomroni. 
Table 4: Sequence information for primers used in RT-qPCR.

\begin{tabular}{|c|c|c|}
\hline Gene & Forward primer ( $5^{\prime}$ to $\left.3^{\prime}\right)$ & Reverse primer ( $5^{\prime}$ to $\left.3^{\prime}\right)$ \\
\hline hSaAURKA & TTCTTCCCAGCGCATTCCTT & TTCCTTTACCCAGAGGGCGA \\
\hline mmuCdc20b & CTCGCCAACGGCATGAAGCAG & CTCCGCAGACAGCCTCTTCACG \\
\hline Cdkn1a & GTGGCCTTGTCGCTGTCTT & GCGCTTGGAGTGATAGAAATCTG \\
\hline Cdkn1b & AGTGTCCAGGGATGAGGAAGCGAC & TTCTTGGGCGTCTGCTCCACAGTG \\
\hline mmuDnah5 & CTGACGGACGCTGGGGACAC & CACTGGGGTGGTCGCCGAAG \\
\hline mmuDnali1 & TTTGGCATGAGGAAGGCACT & CTGGTTGGTCCGTTTCAGGA \\
\hline mmuE2F1 & AACTGGGCAGCTGAGGTGC & CAAGCCGCTTACCAATCCC \\
\hline mmuE2F3 & AAACGCGGTATGATACGTCCC & CCATCAGGAGACTGGCTCAG \\
\hline mmuE2f4 & GCACTGGACACTCGGCCT & TGCACTCTCTCGTGGGGTCG \\
\hline mmuFoxj1 & CCATGCAGACCCCACCTGGCA & GGGCAAAGGCAGGGTGGATGT \\
\hline mmuMcidas & AACAACGAAAAGGAGCCTGGA & GCCGCTTAGGGTCACGATTG \\
\hline mmuMmp9 & CTGGACAGCCAGACACTAAAG & CTCGCGGCAAGTCTTCAGAG \\
\hline mmuMmp12 & GGGCTGCTCCCATGAATGAC & CCAGAGTTGAGTTGTCCAGTTG \\
\hline mmuRfx2 & GACGGCACAAGACACTCTCTG & AGAGTCTCAATCGCCATTTCAAG \\
\hline mmuRfx3 & ATGCAGACTTCAGAGACGGGT & ACTGGCACTTGCTGTACCAC \\
\hline mmuTAp73 & AGCAGAATGAGCGGCAGCGTT & TGTTGGACTCCTCGCTGCCTGA \\
\hline mmu36b4 & GCAGATCGGGTACCCAAC & CAGCAGCCGCAAATGCAG \\
\hline hsa36B4 & TKGCCAGTGTSTGTCTGCAG & CCRCAAAKGCAGATGGATCAG \\
\hline
\end{tabular}

\subsubsection{Chromatin immunoprecipitation}

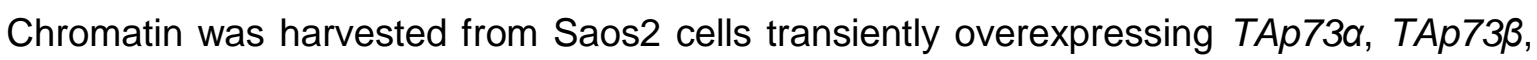
and the control vector pcDNA3.1. ChIP and qPCR was performed as previously described (Nemajerova et al., 2016). Briefly, transfected Saos2 cells were cross-linked with $1.1 \%$ PFA for $30 \mathrm{~min}$. For stopping the cross-linking, $0.125 \mathrm{M}$ glycine was added. After cell lysis using $1 \%$ Triton $\mathrm{X}-100,0,15 \mathrm{M} \mathrm{NaCl}, 1 \mathrm{mM}$ EDTA, $0.1 \%$ SDS, and protease inhibitors, cells were sonicated for 10 min (BioRuptor, Diagenode, high power setting, 30 seconds on/off). An aliquot of chromatin was de-crosslinked as described below and used as input control. Afterwards, chromatin was incubated with Protein A/G plus agarose beads (Santa Cruz) and $2 \mu \mathrm{g}$ of TA-specific p73 antibody or lgG overnight (List of antibodies in Table 1). Following immunoprecipitation, ChIP samples were washed and eluted from beads via a 20 min rotation incubation in $1 \%$ SDS and $0.1 \mathrm{M} \mathrm{NaHCO}$, and de-crosslinked by the 
addition of $0.2 \mathrm{M} \mathrm{NaCl}$ for $4 \mathrm{~h}$ at $65^{\circ} \mathrm{C}$. Subsequently, DNA was purified by the MinElute PCR Purification Kit (Qiagen) and analyzed by realtime PCR using specific primers (List of primers in Table 4). Enrichment levels were determined as the number of PCR products for each gene relative to total input. As described in (Wildung et al., 2018).

Table 5: Sequence information for primers used in ChIP-qPCR.

\begin{tabular}{|c|c|c|}
\hline Gene & Forward primer ( $5^{\prime}$ to $\left.3^{\prime}\right)$ & Reverse primer ( $5^{\prime}$ to $\left.3^{\prime}\right)$ \\
\hline DNAI1 & CCCAAGCGGGGTAATCTCT & CTTGAGGTTGTGGGACTTCAC \\
\hline DNALI1 & CACGCCCGGCAAATTTCTG & CAAGGTGGGCAGATCATGTG \\
\hline FOXJ1 down & CAGCATGCCCAGAAGCTTTG & TCAGGGGCTGCATTCTTCC \\
\hline FOXJ1 end & AGGGCACACTTAGCCTTTG & AGGAGACAAAGGGAGGAGG \\
\hline
\end{tabular}

\subsubsection{In situ hybridization, RNAscope}

Tissue cryosections from mouse embryos were fixed with 4\% PFA for $10 \mathrm{~min}$, acidified (1.3\% Triethanolamine, $0.000065 \% \mathrm{HCl}, 0.25 \%$ Acetic Anhydride) and digested with Proteinase $\mathrm{K}(0.5 \mu \mathrm{g} / \mathrm{ml})$. After pre-incubation of slides with hybridization buffer $(50 \%$ Formamide, 25\% 20x saline-sodium citrate buffer (SSC) pH 4.5, 1\% 0.5 M EDTA, 0.1\% Tween-20, 0.1\% 3-[(3-cholamidopropyl) dimethylammonio]-1-propanesulfonate (CHAPS), $0.1 \mathrm{mg} / \mathrm{ml}$ Heparin, $1 \%$ yeast transfer RNA) for $4 \mathrm{~h}$ at RT, a dioxigenin-labelled LNA-probe (5 pM) against miR449a diluted in hybridization buffer was added for $24 \mathrm{~h}$ at $50^{\circ} \mathrm{C}$ in a chamber humidified with 50\% formamide and $25 \% 20 \times$ SSC buffer. Following two washing steps with pre-warmed $5 \times$ SSC buffer for 5 min and $0.2 x$ SSC buffer for $1 \mathrm{~h}$ at $50^{\circ} \mathrm{C}$, slides were incubated with Buffer B1 (0.1 M TRIS pH 7.5, $0.15 \mathrm{M} \mathrm{NaCl})$ for 10 min at RT and blocked (10\% FCS, 0.05\% Tween-20, 90\% B1 buffer) for $1 \mathrm{~h}$ at RT. The alkaline phosphatase-conjugated anti-dioxigenin antibody (Roche Diagnostics) diluted in blocking solution $(1: 2,000)$ was incubated on the slides overnight at $4^{\circ} \mathrm{C}$. After washing with $\mathrm{B} 1$ buffer, the slides were incubated with buffer B2 (0.1 M TRIS pH 9.5, 0.1 M NaCl, $50 \mathrm{mM}$ $\mathrm{MgCl}_{2}, 0.1 \%$ Tween-20, $2 \mathrm{mM}$ Levamisole) for $10 \mathrm{~min}$. To visualize the in situ hybridization (ISH) signal, slides were incubated with nitroblue tetrazolium chloride $(75 \mathrm{mg} / \mathrm{ml}) / 5$-bromo4-chloro-3-indolyl-phosphate $(50 \mathrm{mg} / \mathrm{ml})$ in B2 buffer for up to 3 days and subsequently mounted with fluorescence mounting medium. As a control, slides were treated the same, but without addition of LNA-probe. Anti-miR449a (ACCAGCUAACAAUACACUGCCA) LNA-probe was purchased from Qiagen.

For RNAscope, TAp73 (probe no. 475741), Mcidas (probe no. 510401-C2), Hes1 (probe no. 417701) and Hes5 (probe no. 400991-C2) were visualized using RNAscope $2.5 \mathrm{HD}$ Duplex Reagent Kit (\#322430, Advanced Cell Diagnostics, Hayward, CA, USA) according to manufacturer's instructions. As described in (Wildung et al., 2018). Experiments were performed by $M$. Lizé and members of the group from $\mathrm{H}$. Zhao). 


\subsection{Microscopy, Video Microscopy}

\subsubsection{Transmission electron microscopy}

For fixation, freshly isolated tissue samples were incubated in $2 \%$ glutaraldehyde in $0.1 \mathrm{M}$ cacodylate buffer (Science Services, München, Germany) at pH 7.4 overnight at $4^{\circ} \mathrm{C}$. Postfixation was performed using $1 \%$ osmium tetroxide diluted in $0.1 \mathrm{M}$ cacodylate buffer. Following pre-embedding staining using 1\% uranyl acetate, and dehydration, tissue samples were embedded in Agar 100 (Plano, Wetzler, Germany). To rule fixation artefacts out, a second embedding technique was performed (only for trachea samples from WT and miR449 ${ }^{-/}$mice). For this, samples were transferred to aluminium platelets of $150 \mu \mathrm{m}$ depth filled with 1-hexadecen (Studer et al., 1989), which were subsequently frozen using a Leica EM HPM100 high pressure freezer (Leica Microsystems). Afterwards, the vitrified samples were placed into an Automatic Freeze Substitution Unit (Leica EM AFS2) and substituted at $-90^{\circ} \mathrm{C}$ in the following solutions: anhydrous acetone, $0.1 \%$ tannic acid (for $24 \mathrm{~h}$ ), and in anhydrous acetone, $2 \% \mathrm{OsO}_{4}$, and $0.5 \%$ anhydrous glutaraldehyde (EMS Electron Microscopical Science, Hatfield, PA, USA) for $8 \mathrm{~h}$. After an additional incubation step for 20 $\mathrm{h}$ at $-20^{\circ} \mathrm{C}$, tissue samples were brought back to $+4^{\circ} \mathrm{C}$, washed with anhydrous acetone, and embedded at RT in Agar 100 at $60^{\circ} \mathrm{C}$ for $24 \mathrm{~h}$. Thin tissue sections (100 nm) were analyzed with a Philips CM 120 BioTwin transmission electron microscope (Philips, Amsterdam, The Netherlands) and micrographs were acquired using a TemCam F416 CMOS camera (TVIPS, Gauting, Germany). As described in (Wildung et al., 2018).

Longitudinal- and cross-sections from motile cilia were evaluated and grouped in normal and abnormal cilia. Abnormal cilia were defined as cilia with a detachment of the ciliary plasma membrane. Images were taken and quantified from at least two different tissue locations from each sample. Experiment was performed by D. Riedel.

\subsubsection{Mucociliary transport assay}

Freshly isolated vital tracheae were longitudinally cut using a microtome (VT1200S, Leica Microsystems) and rinsed with Ringer`s solution containing $98 \mathrm{mM} \mathrm{NaCl}, 2 \mathrm{mM} \mathrm{KCl}, 1 \mathrm{mM}$ $\mathrm{CaCl}_{2}, 2 \mathrm{mM} \mathrm{MgCl}$, $5 \mathrm{mM}$ glucose, $5 \mathrm{mM}$ sodium pyruvate, $10 \mathrm{mM}$ Hepes $(230 \mathrm{mOsm}, \mathrm{pH}$ 7.8). Fluorescent microspheres (FluoSpheres ${ }^{\circledR}, 0.17 \mu \mathrm{m}$, PS-Speck, Invitrogen, Thermo Fisher Scientific) diluted in Ringer`s solution were added onto the multiciliated epithelium of the tracheae. In areas of $280 \times 280 \mu \mathrm{m}(512 \times 512$ pixels $)$ the movement of the fluorescent microspheres was recorded using a custom-built confocal line illumination microscope (Junek et al., 2009) at $61 \mathrm{~Hz}$ for 2,000 frames. Note that only areas with beating cilia were imaged. For each frame, particles were detected and tracked using functions previously described (Crocker, J. C., et al., 1996). Absolute full-trajectory velocities along the focal plane were combined within one measurement. Averaged velocities of independent measurements from different tissue areas and different mice were assigned into the 
following groups: WT, miR449 ${ }^{-}$, and diffusion. Diffusion measurements were obtained from non-living dried tracheae and pooled from WT and miR449־ mice, since they were identical. Individual trajectory velocities from all measurements within the respective group were included to obtain particle velocity distributions. All data evaluation was carried out using a custom software written in Matlab (MathWorks, Natick, MA, USA). Age-matched WT and miR449 mice (12 weeks) were used. Data evaluation was performed by Mihai Alevra.

\subsubsection{Video microscopy of spermatozoa and smooth muscle contraction of fallopian tubes}

The murine FT and testis connected to the epididymis were dissected and transferred to DMEM. To image spermatozoa, the epididymis was separated from the testis and vas deferens. An incision was made at the distal end to release spermatozoa. Spermatozoa as well as the peristaltic contraction of the FT was imaged with an inverse microscope. As described in (Wildung et al., 2018).

\subsubsection{Imaging of cilia-generated bead-flow and cilia beating in the brain ventricular system}

Mouse brains were dissected and transferred to DMEM 21063 (Gibco, Thermo Fisher Scientific). Coronal slices containing the lateral ventricle, ventral third ventricle, aqueduct, and fourth ventricle were prepared by using a coronal adult brain matrix (ASI Instruments, Warren, MI, USA). The ventral third ventricle was processed further as previously described (Faubel et al., 2016). The tissue explant was placed in DMEM containing fluorescent latex beads (Fluoresbrite Multifluorescent 1.0 micron Microspheres, Polysciences, Warrington, $P A, U S A)$. Movement of fluorescent beads along the ventricular wall and within the ventricular lumen was observed by fluorescence microscopy using a DMR (Leica Microsystems) upright microscope with an epifluorescence lamp. Ciliary beating was observed by differential interference contrast microscopy using the same set-up. Bead movement was recorded using a high-speed camera operated by MultiRecorder Software (Cascade II-512, Photometrics, Tucson, AZ, USA) and analyzed using the ImageJ software (Schindelin et al., 2012). As described in (Wildung et al., 2018). Described and performed by $A$. Guenther.

\subsection{Statistical Analysis}

For the data used in the manuscript "Transcription factor TAp73 and microRNA-449 cooperate in multiciliogenesis", a one-tailed, unpaired Student's test assuming normal distribution was used to calculate statistical significance for pairwise comparisons. Luciferase assay statistics were assessed using one-way ANOVA assuming normal distribution followed by Dunnett's multiple comparison tests. Statistical analyses were carried out using the GraphPad Prism software. The following indications of significance 
were used throughout the manuscript: ${ }^{*} p<0.05,{ }^{* *} p<0.01,{ }^{* * *} p<0.001$. Results are shown

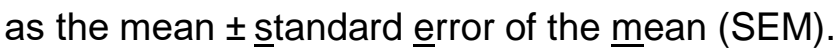

For the data used in the manuscript "miR449 protects airway cilia and healthy lung aging, thereby preventing COPD", a two tailed, unpaired or paired $t$-test with Welch-correction (used only for unequal variances) was used to calculate statistical significance for pairwise comparisons. Except for Figure $5 \mathrm{~d}$ and $6 \mathrm{c}$ were a one tailed unpaired $t$-test and a two tailed paired $t$-test were applied, respectively. Normal distribution was assumed for all analyzed data. Statistical analyses were carried out using the GraphPad Prism software.

The following indications of significance were used throughout the manuscript: ${ }^{*} \mathrm{p}<0.05$,

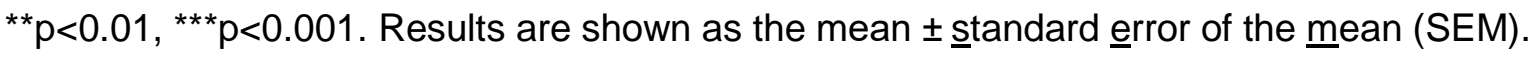




\section{RESULTS}

\subsection{Chapter 1: Transcription factor TAp73 and microRNA-449 cooperate in multiciliogenesis}

Merit Wildung ${ }^{1, *}$, Tilman Uli Esser ${ }^{1, *}$, Katie Baker Grausam ${ }^{2,3}$, Cornelia Wiedwald ${ }^{1}$, Larisa Volceanov-Hahn ${ }^{1}$, Dietmar Riedel ${ }^{4}$, Sabine Beuermann ${ }^{1}$, , Li Li ${ }^{2}$, Jessica Zylla ${ }^{2}$, Ann-Kathrin Guenther $^{5}$, Magdalena Wienken ${ }^{6}$, Evrim Ercetin ${ }^{1}$, Zhiyuan Han ${ }^{7}$ Felix Bremmer ${ }^{8}$, Orr Shomroni ${ }^{9}$, Stefan Andreas ${ }^{1}$, Haotian Zhao ${ }^{2,3,7, \#}$ and Muriel Lizé ${ }^{1, \#}$

* = equal contribution; \# = corresponding authors

1) Molecular \& Experimental Pneumology Group, Clinic for Cardiology and Pneumology, University Medical Center Goettingen, Germany

2) Cancer Biology and Immunotherapeutics Group, Sanford Research, Sioux Falls, South Dakota, USA

3) Division of Basic Biomedical Sciences, University of South Dakota, Sanford School of Medicine, Vermillion, South Dakota

4) Electron Microscopy, Max-Planck-Institute for Biophysical Chemistry, Goettingen, Germany

5) Department of Genes and Behavior, MPI for Biophysical Chemistry, Goettingen, Germany

6) Institute of Molecular Oncology, University Medical Center Goettingen, Germany

7) Department of Biomedical Sciences, New York Institute of Technology College of Osteopathic Medicine, Old Westbury, New York, USA

8) Institute of Pathology, University Medical Center Goettingen, Goettingen, Germany

9) Microarray and Deep-Sequencing Core Facility, University Medical Center Goettingen, Germany

This manuscript is published on a preprint server for biology (BioRxiv) and online available at https://doi.org/10.1101/273375.

This manuscript has been peer-reviewed and is currently in revision (October 2018).

\section{Contribution to manuscript:}

M. W. conducted experiments and data analysis for the following figures:

Figure 15a, b; Figure 16b-d; Figure 17a, c; Figure 18b-d, Figure 19b; Figure 23b-d; Figure 25a, d; Figure 27; Supplemental Figure 2a, b, d; Supplemental Figure 3a; Supplemental Figure 5c, d; Supplemental Figure 7a; Supplemental Figure 9c, d, Supplemental Figure 12a, $b$, Supplemental Figure $13 a, b$.

$\mathrm{M}$. W. arranged the figures and contributed to manuscript writing.

D.R. performed electron microscopy analysis. Ma.W. contributed to the validation of TAp73 targets by ChIP. E.E. and F.B. contributed IF analysis of human epididymis. E.E. performed cilia quantification on trachea. L.V-H. and S.B. contributed to Western blot analysis of different tissues. C.W. maintained mice, performed RNA isolation and qPCRs. K.B.G., J.Z., L.L., Z.H. and H.Z. contributed brain analyses. O.S. analyzed small RNA sequencing data. S.A. contributed to interpretation and supported the group. M.L. developed the project, interpreted the data, designed and coordinated the experiments to complete this study. 


\subsubsection{TAp73 is expressed in diverse multiciliated epithelia}

We and others previously showed that TAp73, which is expressed in respiratory epithelia, controls airway multiciliogenesis (Marshall et al., 2016; Nemajerova et al., 2016). However, little is known about the expression and function of TAp73 in other MCC types. To address this, we performed immunostaining and in situ hybridization studies and demonstrated TAp73 expression in EDs, FTs, and ependymal and CP epithelial cells in the brain as well as the testis as previously described (Hamer et al., 2001; Holembowski et al., 2014; Inoue et al., 2014) (Figure 14a - f, Supplemental Figure 1a).

a

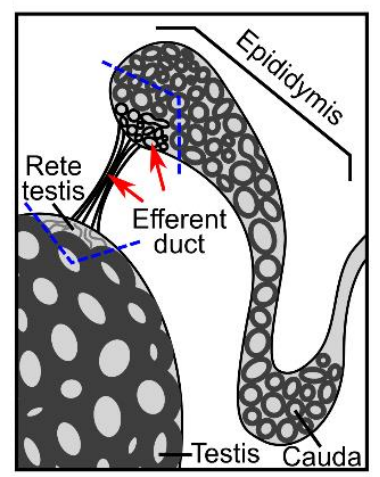

b

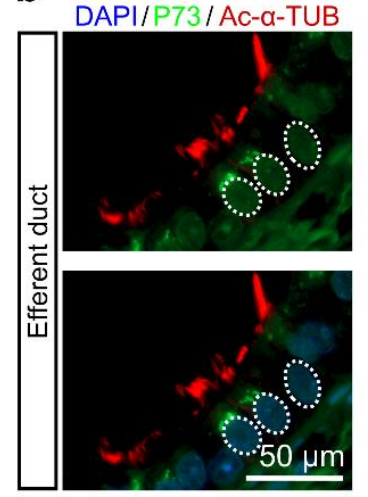

C

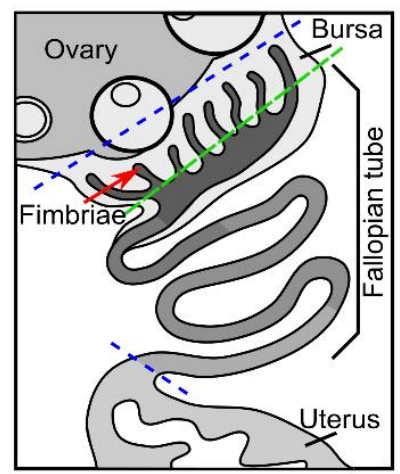

d

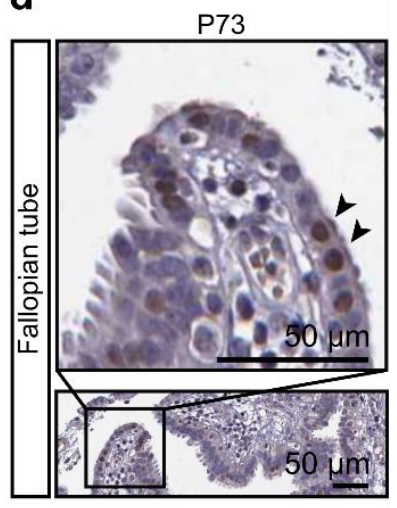

e

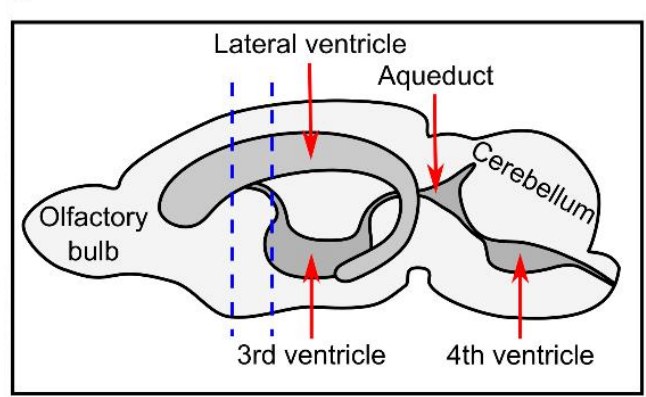

f Lateral ventricle

4th ventricle

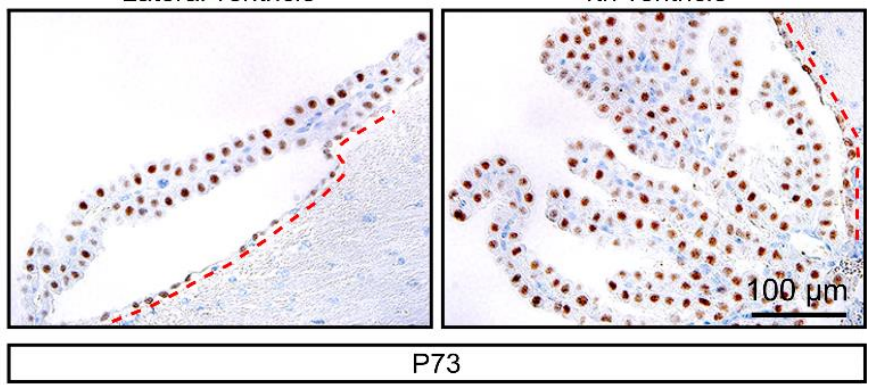

Figure 14: TAp73 is expressed in diverse multiciliated epithelial cells.

a) Schematic illustration of the EDs (arrows) that connect the testis with the epididymis. Blue dotted lines indicate the region used for protein and RNA analyses. b) Expression of P73 (green) and the axonemal cilium marker acetylated-alpha-tubulin (Ac-a-TUB, red) is shown in the human ED. White bracket circles delineate P73 staining. DAPI staining (blue) marks nuclei. c) Schematic illustration of the FT that connects ovary and uterus. Green dotted line illustrates the region used for IF analysis. Blue dotted lines display cuttings site for protein and RNA analyses. Arrow points to ciliated fimbriae. d) Expression of P73 is shown in the human FT. Upper panel depicts a magnification of the boxed region in the lower panel. Arrowheads mark $\mathrm{P}^{+} 3^{+}$cells. Images were obtained from Human Protein Atlas:

(https://www.proteinatlas.org/ENSG00000078900-TP73/tissue/fallopian+tube). e) Schematic illustration of murine brain ventricles (arrows). Blue dotted lines indicate the position of coronal brain slices used for protein and RNA analyses. f) Expression of TAp73 is shown in the lateral and 4th ventricle of WT mice. Red dotted lines demarcate ventricles lined with ependymal cells. Notice that both ependymal and CP epithelial cells express TAp73. 
RT-qPCR and western blot analyses showed that, among different multiciliated epithelia, FTs and EDs exhibited higher levels of TAp73 expression than the ventricles of the brain (Figure 15a-c). Together, our results demonstrate robust TAp73 expression in different multiciliated epithelia.

a

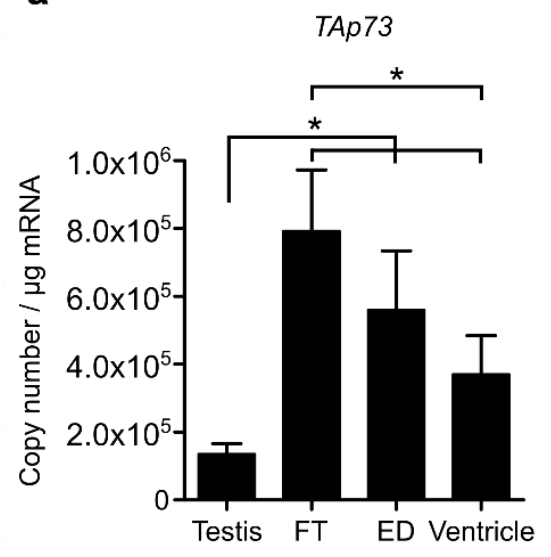

b
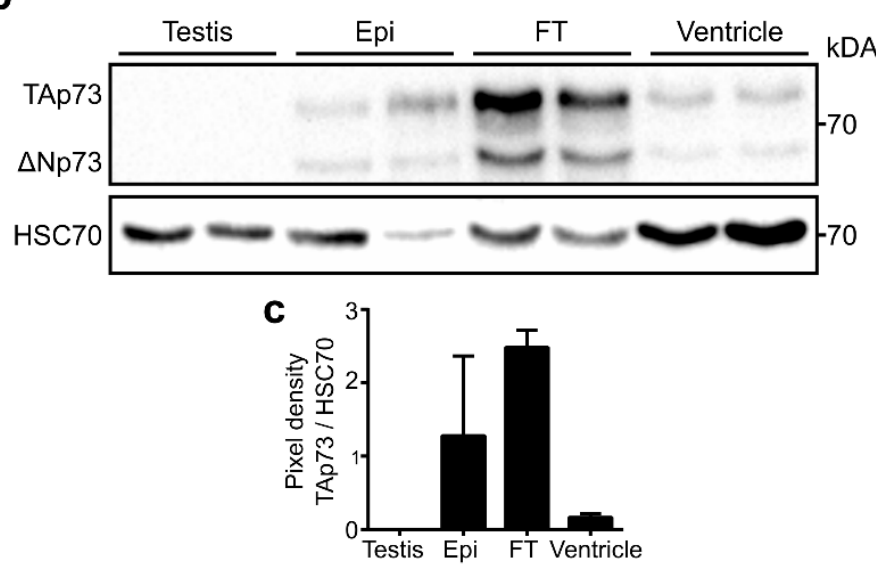

Figure 15: Expression levels of TAp73 vary within different multiciliated tissues.

a) Absolute quantification of the expression of TAp73 in the testis, FT, epididymis (Epi), and the brain ventricle of WT mice using a murine TAp73 plasmid standard curve. Expression levels are shown relative to TAp73 expression in testis (testis: $n=3, \mathrm{FT}: n=4$, ED: $n=6$, ventricle: $n=4$ ). b) Western blot analysis of the expression of TAp73, and P73 isoform lacking N-terminal ( $\triangle \mathrm{Np} 73$ ) in Epi, FT, and brain ventricle of WT mice. HSC70 serves as a loading control. Of note, TAp73 was not detected in testis. c) Quantitation of the immunoblot bands shown in (b). Data are presented as the mean \pm SEM. Confer (Cf.) Figure 14a, c, and e for cutting area of ED, FT, and ventricle, respectively.

\subsubsection{TAp73 is crucial for the molecular circuit of multiciliogenesis in efferent ducts}

Loss of TAp73 leads to male infertility that has been attributed to defective germ cell maintenance during spermatogenesis, which results in a massive reduction of germ cells in the testis (Holembowski et al., 2014; Inoue et al., 2014). Interestingly, we detected spermatozoa in the testis of TAp73 KO mice, though at a markedly reduced level (Supplemental Figure 2a). However, spermatozoa did not reach the cauda epididymis (Figure 16a), indicative of a defect in the conduit (EDs) connecting the testis and the epididymis. The multiciliated epithelium of the EDs contributes to spermatozoa transport from the testis to the epididymis by facilitating testicular fluid circulation, fluid reabsorption, and spermatozoa concentration (Hess, 2002; Hess, 2015), all essential aspects of male fertility (Mendive et al., 2006; Dacheux and Dacheux, 2013; Danielian et al., 2016). As TAp73 is expressed in MCCs of the ED epithelium we analyzed the EDs in TAp73 KO mice. Indeed, although no gross morphological differences were observed between WT and TAp73 KO EDs (Figure 16a), immunofluorescent staining of the cilia components acetylated-alpha-tubulin (Ac- $\alpha-T U B)$ and the axonemal dynein DNAl1 showed a dramatic 
reduction in the number and length of motile cilia in the EDs of TAp73 KO mice (Figure 16b and c), resembling the loss of airway cilia in these animals (Nemajerova et al., 2016). Ultrastructural studies supported the observation of a ciliary defect in TAp73 KO EDs and additionally indicated an increased BB mislocalization (Figure 16d, Supplemental Figure $2 b)$.

a

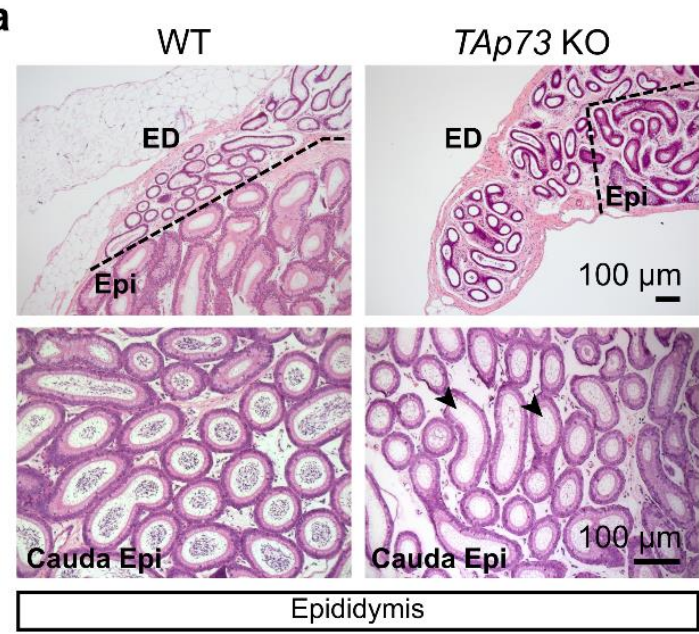

C

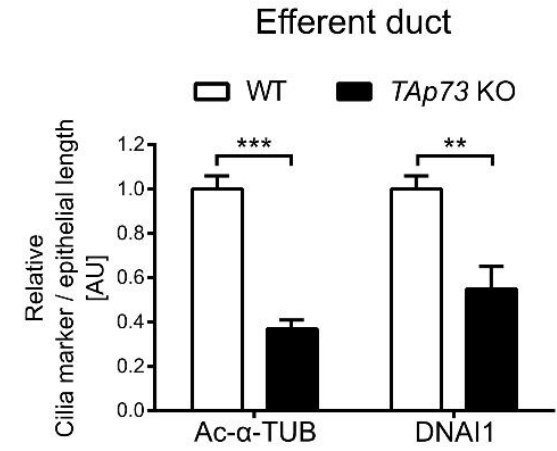

d

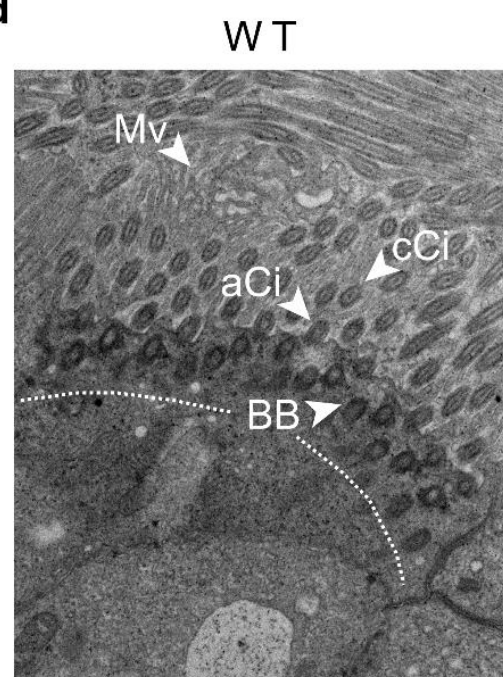

b
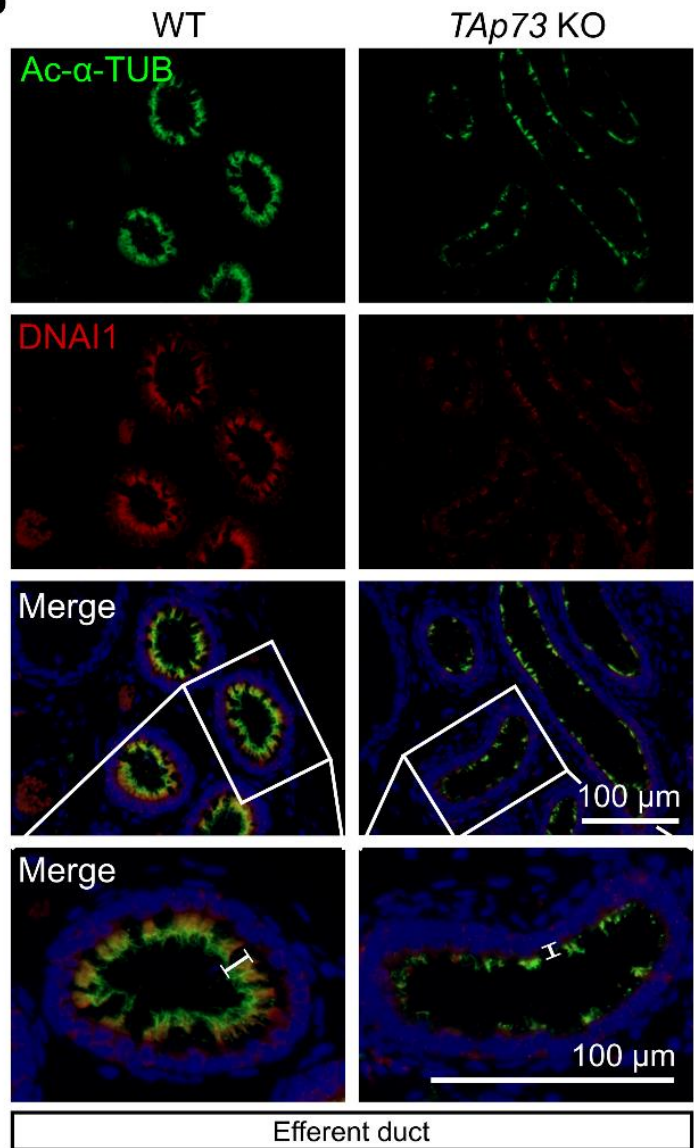

Efferent duct

Figure 16. 
Figure 16: TAp73 deficiency results in loss of motile cilia in the efferent ducts.

a) Representative images of H\&E staining of ED and epididymis (Epi) sections in WT and TAp73 KO mice. Bracket lines demarcate the border of ED and Epi. Notice the lack of mature spermatozoa in the cauda Epi in TAp73 KO mice (arrowhead). b) Expression of the cilia markers Ac-a-TUB (green) and axonemal dynein DNAl1 (red) in EDs of WT and TAp73 KO mice. DAPI staining (blue) labels nuclei. Boxed regions are magnified in the bottom panel. Notice that TAp73 KO mice have less abundant and shorter cilia (white bars) compared to their WT counterparts. c) Quantitation of Ac-a-TUB and DNAl1 signals normalized to epithelial length (WT: $n=6$ images from 3 mice; TAp73 KO: $n=11$ images from 4 mice). Data are presented as the mean \pm SEM and relative to WT group. d) Representative photomicrographs of transmission electron microscopy (TEM) of a MCC in the ED from WT and TAp73 KO mice. BB = basal body, $\mathrm{aCi}=$ axoneme of cilium, $\mathrm{cCi}=$ cross section of cilium, $\mathrm{Mv}=$ microvilli.

We and others previously demonstrated that TAp73 promotes multiciliogenesis by transcriptionally activating a network of pro-ciliogenic genes (e.g. Foxj1) (Marshall et al., 2016; Nemajerova et al., 2016). Here, we confirmed this interaction and identified two new TAp73 targets as ChIP followed by qPCR revealed a significant enrichment of TAp73 in genomic loci of the axonemal dyneins DNALI1 and DNA/1, and as previously reported FOXJ1 (Figure 17a, Supplemental Figure 3a) (Nemajerova et al., 2016). Accordingly, transcript levels of Dnali1, Foxj1, Rfx2, and Rfx3 and protein levels of DNAI1 and DNALI1 were strongly reduced in male reproductive ducts of TAp73 KO animals (Figure $17 \mathrm{~b}$ and c, Supplemental Figure $2 \mathrm{c}$ and $\mathrm{d}$ ).

In summary, our results indicate that TAp73 directs Dnali1 and Dnai1 in addition to known critical nodes including Foxj1, Rfx2, and Rfx3 to mediate multiciliogenesis in EDs (Figure $27 \mathrm{a}$ and $\mathrm{b})$. Thus, these additional defects in the multiciliated epithelium of the EDs may contribute to male infertility in TAp73 KO mice. 
a

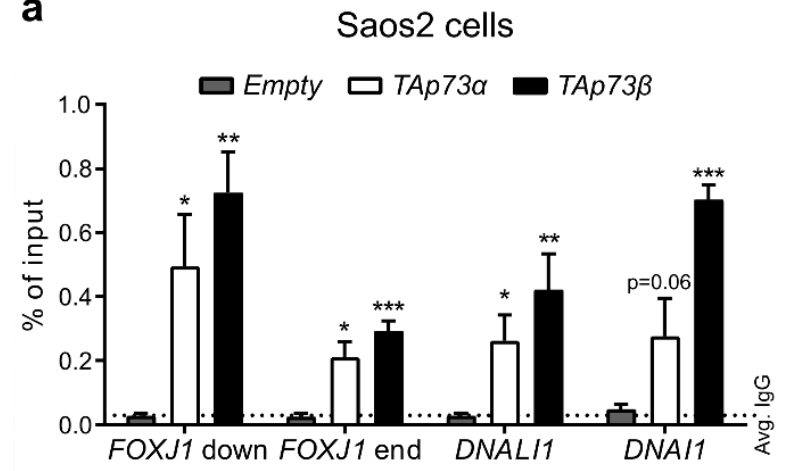

b

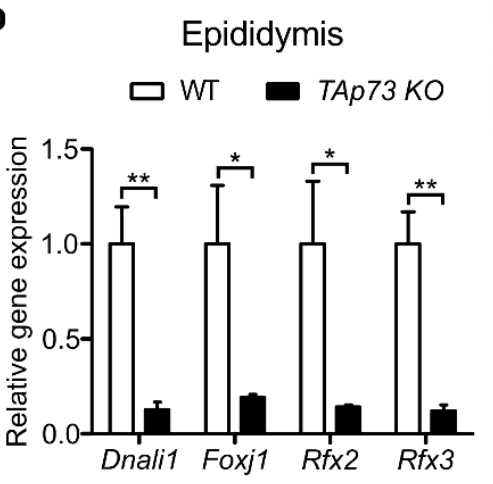

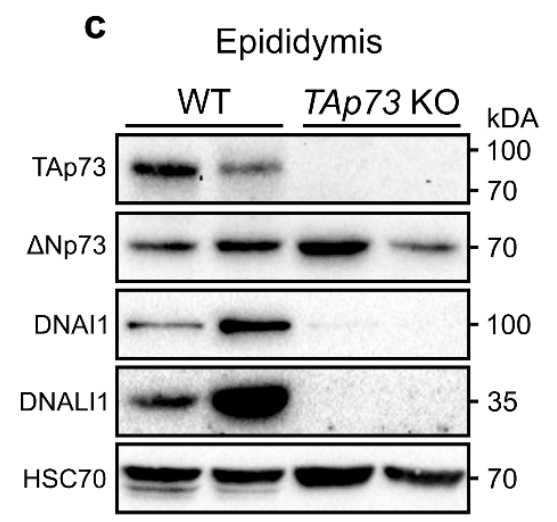

Figure 17: TAp73 controls multiciliogenesis in the male reproductive tract.

a) ChIP was performed in Saos2 cells transfected with TAp73a, TAp73 $\beta$, and empty control vector. Binding of TAp73a and TAp73 $\beta$ to genomic regions of FOXJ1, axonemal dyneins DNALI1 and DNAl1 was compared to that of the empty vector using RT-qPCR ( $n=3$ for each antibody/gene pair, except for DNALI1 [ $n=4]$ ). Genomic regions examined are illustrated in Supplemental Figure 3 (Koeppel et al., 2011). b) RT-qPCR analysis of Dnali1, Foxj1, Rfx2, and Rfx3 expression in EDs from WT and TAp73 KO mice relative to the WT group (WT: $n=4$ for Dnali1, Foxj1, and Rfx3, $n=3$ for Rfx2; TAp73 KO: $n=3$ ). c) Immunoblot analysis of the expression of TAp73, $\triangle$ Np73, DNALI1, and DNAl1 in epididymis of WT and TAp73 KO animals. HSC70 serves as a loading control. Cf. Figure 14a for cutting area of EDs. Data are presented as the mean \pm SEM.

\subsubsection{TAp73-driven transcriptional network regulates multiciliogenesis in fallopian tubes}

Infertility in female TAp73 mutant mice is associated with defects of oocyte release from the ovary and progression along the FTs (Tomasini et al., 2008; Tomasini et al., 2009). However, it remains unclear how TAp73 loss affects gamete transport in the female reproductive tract. Gamete transport along the FT epithelium depends on efficient motile cilia beating and smooth muscle contraction (Ezzati et al., 2014), whereas the latter one was not affected by TAp73 loss (video not shown). As TAp73 is expressed in MCCs of the FT epithelium (Figure 14d, Supplemental Figure 1a), we examined the multiciliated epithelium of the FTs in TAp73 KO animals to identify the mechanism behind the impaired gamete transport. Despite normal tubal morphology (Figure 18a), analysis of Ac- $\alpha-$ TUB and DNAI1 expression showed reduced cilia coverage of the oviduct epithelium in TAp73 mutant mice (Figure $18 \mathrm{~b}$ and $\mathrm{c}$ ). Consistently, transmission electron microscopy (TEM) 
demonstrated ciliary defects including mislocated BBs in the FTs of TAp73 KO mice (Figure 18d, Supplemental Figure 4a).
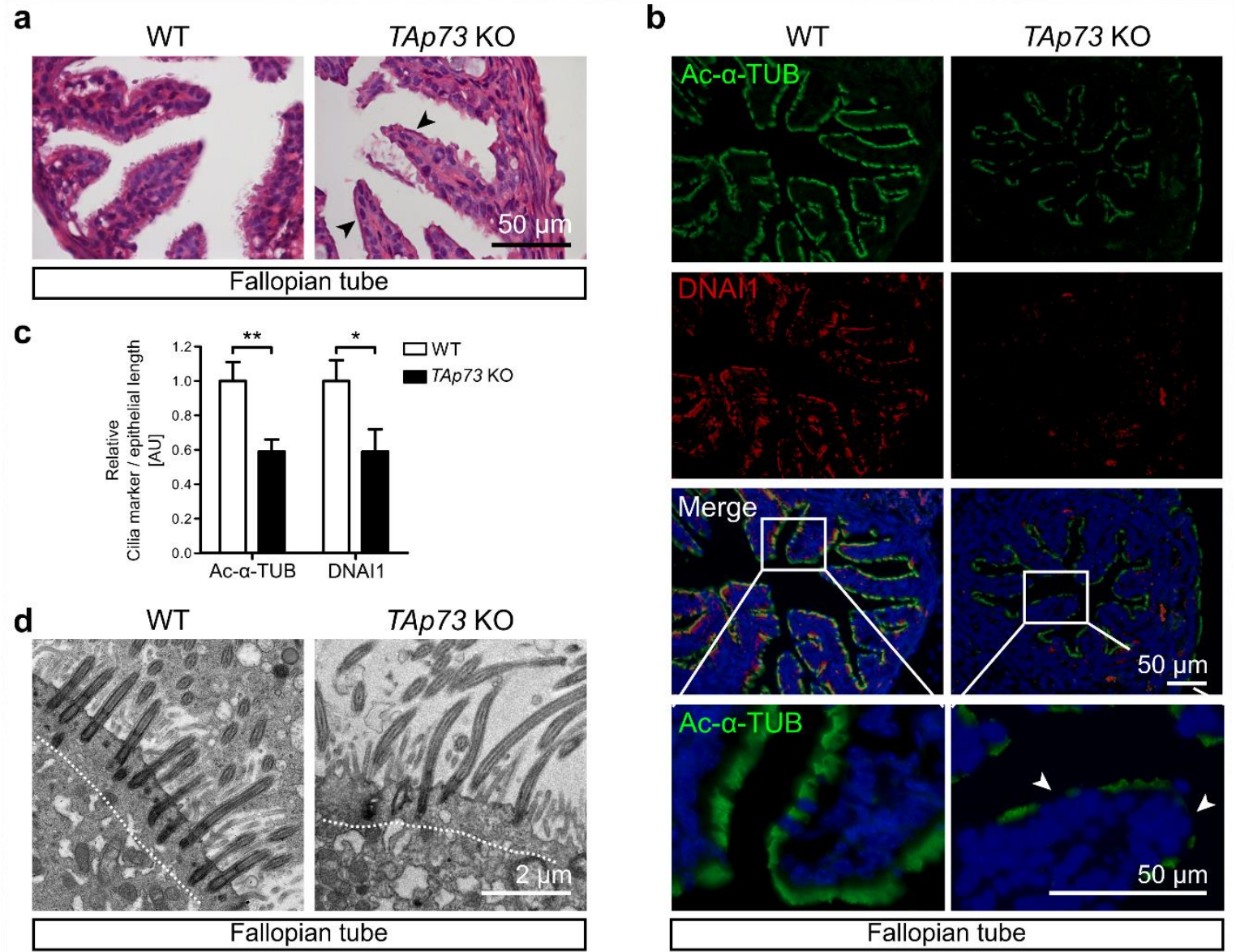

Figure 18: Loss of TAp73 results in reduced ciliation in the oviducts.

a) Representative H\&E images of the FT of WT and TAp73 KO animals. b) Expression of Ac-a-TUB (green) and DNAl1 (red) in the FT of WT and TAp73 KO mice. DAPI staining (blue) labels nuclei. Boxed regions are magnified in bottom panel. In contrast to multiciliated FTs in WT, TAp73 KO mice exhibit segments devoid of cilia (arrowheads). c) Quantitation of Ac-a-TUB and DNAI1 IF signals normalized to epithelial length (WT: $n=6$ images from 4 mice; TAp73 KO: $n=6$ images from 3 mice). Data are presented as the mean \pm SEM and relative to the WT group. d) Representative photomicrographs of TEM of a MCC in the FT from WT and TAp73 KO mice.

Similar to the EDs of TAp73 KO mice, mRNA expression levels of Dnali1, Foxj1, Rfx2, and Rfx3 were reduced in TAp73 KO FTs (Figure 19a), which was accompanied by declined protein expression of FOXJ1, DNAI1, DNALI1 (all expressed in the human FTs, cf. Supplemental Figure 4b), and gamma-tubulin ( $\gamma$-TUB, BB marker) (Figure 19b). Of note, the loss of the TAp73-driven multiciliogenesis program in FTs was less pronounced as in the EDs.

Taken together, these data strongly suggest that loss of TAp73 leads to reduced multiciliogenesis in the oviducts that may contribute to defective oocyte transport and infertility (Tomasini et al., 2008) (Figure 27a and c). 
a

Fallopian tube

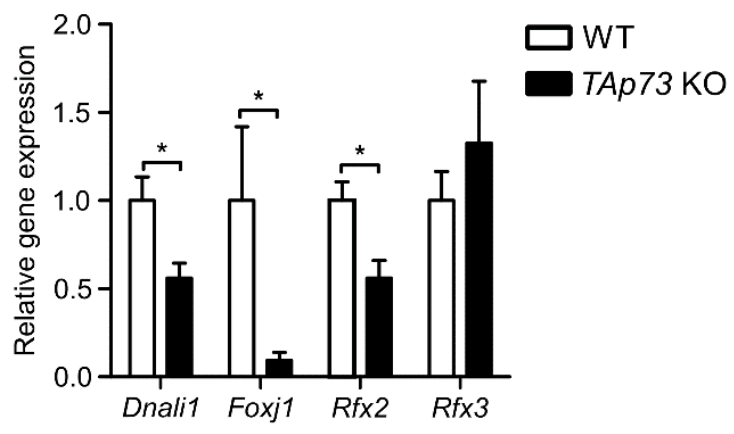

b

Fallopian tube

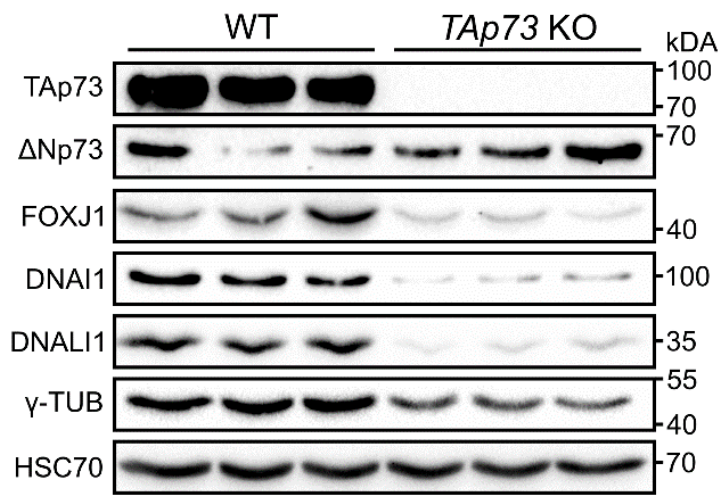

Figure 19: TAp73 controls multiciliogenesis in the oviducts.

a) RT-qPCR analysis of Dnali1, Foxj1, Rfx2, and Rfx3 expression in oviducts from WT and TAp73 KO mice $(n=3)$. Data are presented as the mean \pm SEM and relative to the WT group. b) Immunoblot analysis of the expression of TAp73, $\triangle$ Np73, FOXJ1, DNALI1, DNAI1, and $y$-TUB in oviducts from WT and TAp73 KO animals. HSC70 serves as a loading control. Cf. Figure $14 \mathrm{c}$ for cutting sites used to isolate FTs.

\subsubsection{Ciliary function in the brain is unaltered in the absence of TAp73}

Given TAp73 expression in ependymal and CP epithelial cells in adulthood (Figure 14f), we further evaluated TAp73 expression during brain development. Immunofluorescent studies showed that proliferative progenitors $\left(\mathrm{KI}-67^{+}\right)$are present in the hindbrain roof plate, whereas post-mitotic cells expressing Aquaporin 1 (AQP1) (Huang et al., 2009b; Li et al., 2016) are detected in CP epithelium (KI-67/AQP1 ${ }^{+}$) (Figure 20a). Notably, a portion of the roof plate exists between the progenitors and $\mathrm{CP}$ epithelium that remains undifferentiated after cell cycle exit (KI-67/AQP1') (Figure 20a). In contrast to progenitors with a solitary primary cilium, the "transition" zone is comprised of MCCs that exhibit P73 expression at day E14.5 (Figure 20b).

a

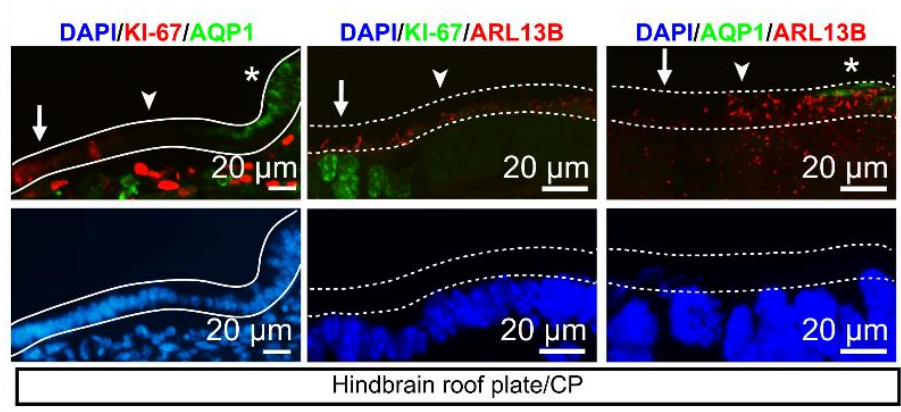

b

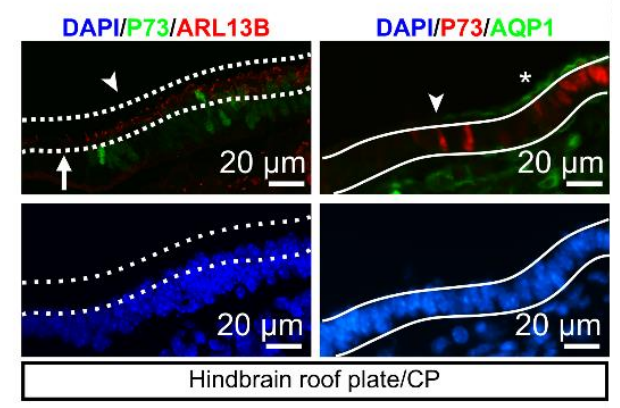

Figure 20. 
Figure 20: TAp73 is expressed during brain development.

a) The expression of KI-67 (green, red), Aquaporin (AQP1, green), and ADP-ribosylation factor-like $13 \mathrm{~b}$ (ARL13B) (red) in WT hindbrain roof plate/CP at embryonic day (E) 14.5. Notice that $\mathrm{KI}-67^{+}$roof plate progenitors, and $A Q P 1+C P$ epithelial cells are spatially separated. ARL13B labels monociliated roof pate progenitors and multiciliated CP epithelial cells. White lines demarcate roof plate epithelium (KI-67+/AQP1arrows), CP epithelium (KI-67-IAQP1+, asterisks), and "transition zone" (KI-67-IAQP1-, arrowheads) in which MCCs appear. Dotted lines mark apical surface with cilia. DAPI staining (blue) labels nuclei. b) Expression of TAp73 (green, red), AQP1 (green), and ARL13B (red) in WT hindbrain roof plate/CP at day E14.5. Dotted lines mark apical surface of roof plate (TAp73-, arrow) and transition zone (TAp73+, arrowhead). White lines mark transition zone (TAp73+/AQP1-, arrowhead) and $\mathrm{CP}$ epithelium (TAp73+/AQP1 ${ }^{+}$, asterisk). DAPI staining (blue) labels nuclei.

The expression of TAp73 in the ventricles of the brain, together with recent studies suggesting the role of E2F4/MCIDAS (which activate the transcription of TAp73 (Nemajerova et al., 2016)]) in brain multiciliogenesis (Kyrousi et al., 2015; Kyrousi et al., 2016), led us to examine the function of TAp73 in MCCs in the ependyma and CP. Gene expression studies confirmed loss of P73 expression in ependymal cells and the CP from TAp73 KO mice (Figure 21a), whereas morphological analysis revealed no apparent defect in these cells (Supplemental Figure 5a). Next, we performed immunostainings for the cilia markers ADP-ribosylation factor-like 13b (ARL13B; (Caspary et al., 2007)]), Ac-a-TUB, and DNAI1 in the 4th and lateral ventricle to assess the effect of TAp73 deficiency on brain multiciliogenesis. In contrast to FTs and EDs, the number and length of cilia from ependymal and CP cells in TAp73 KO mice were similar to those of WT mice (Figure 21b and c, Supplemental Figure $5 b-d)$.

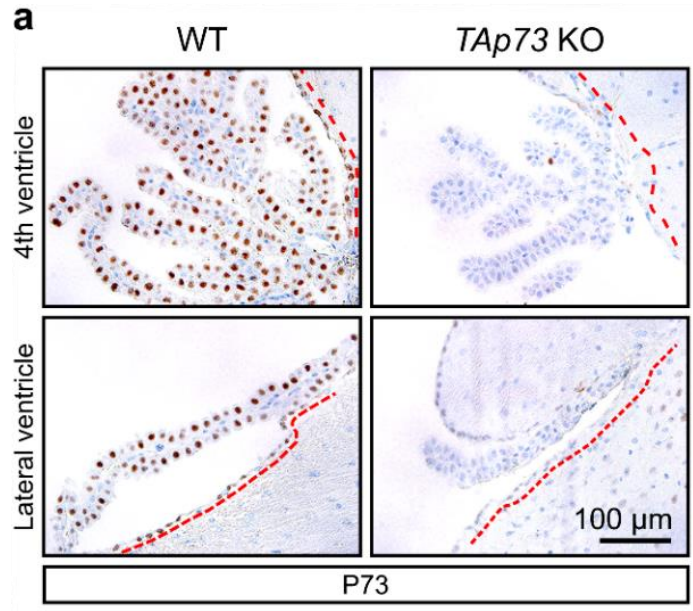

C

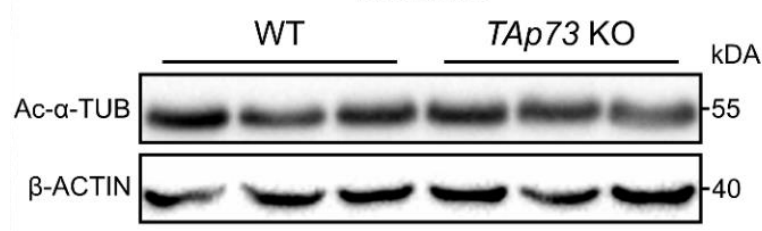

b
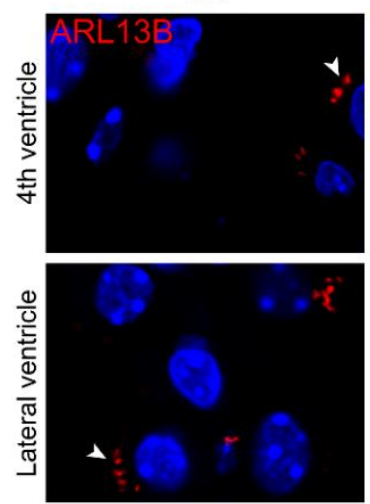

4 th ventricle

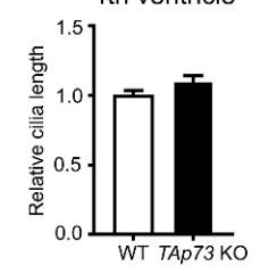

TAp73 KO

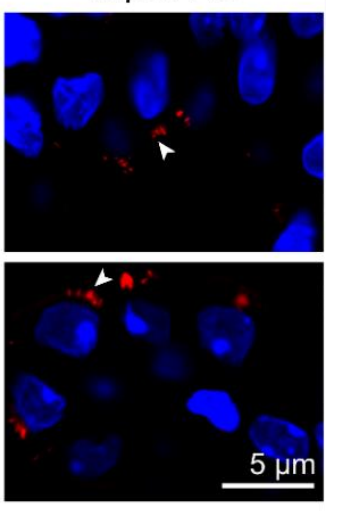

Lateral ventricle

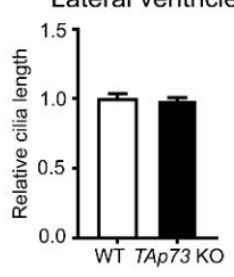

Choroid plexus

Figure 21. 
Figure 21: Ablation of TAp73 does not results in loss of cilia in the ventricles of the brain.

a) Representative images of TAp73 expression in ependymal and CP epithelial cells in the 4th and lateral ventricle from WT and TAp73 KO mice. Red dotted lines mark ventricles lined with ependymal cells. Notice that TAp73 expression is lost in TAp73 KO animals. b) Expression of the cilia marker ARL13B (red) in CP epithelial cells in WT and TAp73 KO mice. DAPI staining (blue) labels nuclei. Quantitation of average cilia length is shown in the lower graphs (WT: $n=12$ cells [4th] and 9 cells [lateral ventricle] from 2 mice; TAp73 KO: $n=17$ cells [4th] and 15 cells [lateral ventricle] from 3 mice). Data are presented as the mean \pm SEM and relative to the WT group. c) Immunoblot analysis of the expression of Ac-a-TUB in brain ventricles from WT and TAp73 KO animals. Cf. Figure $14 \mathrm{e}$ for brain cutting area. $\beta$-ACTIN serves as a loading control.

Moreover, transcript analysis demonstrated comparable expression of Dnali1 and Foxj1, whereas increased $R f x 2$ and $R f x 3$ mRNA levels were observed in brain ventricles of TAp73 $\mathrm{KO}$ mice (Figure 22a). Consistently, ciliary beating and bead flow in the cerebrospinal fluid appeared unaffected in TAp73 KO animals (Figure 22b, video not shown). In addition, no significant difference was observed in expression markers for CP epithelial differentiation between control and TAp73 KO animals (Supplemental Figure 6a-d). To conclude, our results indicate that the function of MCCs in the brain remains intact despite TAp73 loss.

a

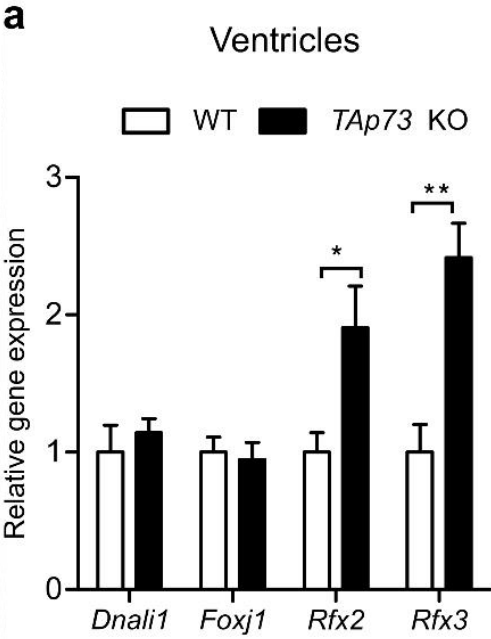

b

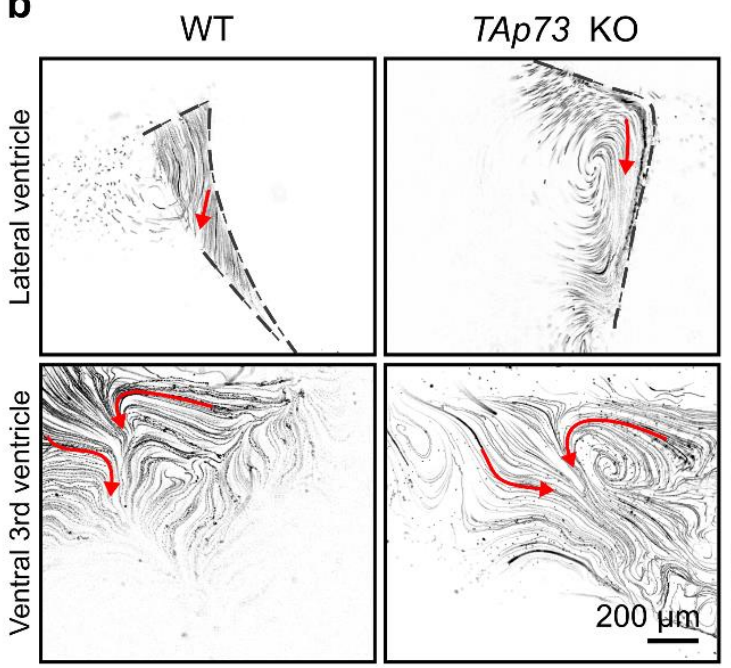

Figure 22: TAp73 is dispensable for brain multiciliogenesis.

a) RT-qPCR analysis of Dnali1, Foxj1, Rfx2, and Rfx3 expression in brain ventricles from WT and TAp73 KO mice (WT: $n=3$; TAp73 KO: $n=4$ ). Cf. Figure $14 \mathrm{e}$ for cutting sites used for brain analysis. Data are presented as the mean \pm SEM and relative to the WT group. b) Quantitation of the movement of fluorescent beads along the ventricular system. Images of maximum intensity projections of representative movies of the lateral and the ventral 3rd ventricles (WT: $n=2$; TAp73KO: $n=3$, TAp73 heterozygous: $n=1$ ). Red arrows mark the direction of the bead flow. Bracket lines depict ependymal layer lining the ventricles.

\subsubsection{TAp73 regulates $m i R-34 / 449$ family members in diverse multiciliated tissues}

In addition to the direct transcriptional regulation, TAp73 influences post-transcriptional mechanisms via miRNAs (Nemajerova et al., 2016). Sequencing of small RNA species from brain ventricles in TAp73 KO mice revealed a significant reduction in miR34b,c (Figure 23a), reminiscent of findings in the airways of these animals (Nemajerova et al., 2016). 
Remarkably, our analysis also showed a strong induction of the miR449 cluster (Figure 23a) that works together with miR34b,c to regulate multiciliogenesis in different tissues across species (Lizé et al., 2010a; Marcet et al., 2011; Song et al., 2014; Otto et al., 2017) and is predominately detected in the CP (Redshaw et al., 2009) (Figure 23b). RT-qPCR analysis confirmed the deregulation of the miR-34/449 family members and revealed a 10-fold increase of miR449 upon TAp73 loss in the ventricles (Figure 23c). The interaction between TAp73 and the miR-34/449 family members was further validated in other multiciliated tissues, namely the ED, FT, and trachea (Figure 23d, Supplemental Figure 7a). Taken together, these results demonstrate a conserved reaction from the pro-ciliogenic miR34/449 family following TAp73 loss in MCCs.

a

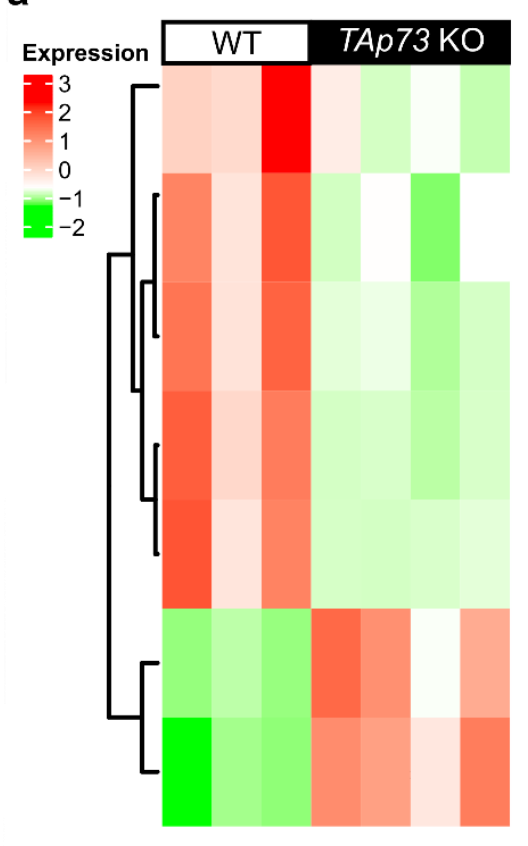

b
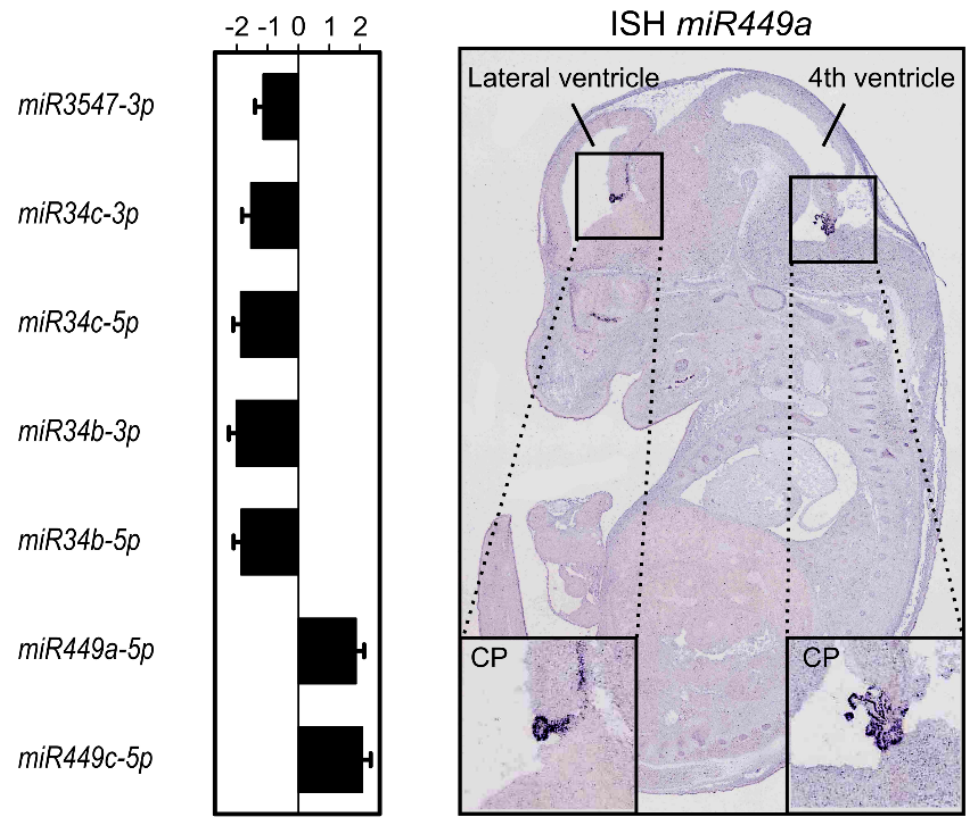

d

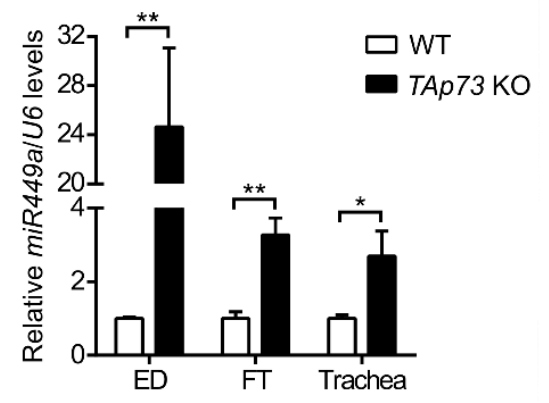

Figure 23. 
Figure 23: TAp73 functions through miR-34/449 family in brain multiciliogenesis.

a) Hierarchical clustering of differentially expressed miRNAs in brain ventricles of WT and TAp73 KO mice (WT: $n=3$; TAp73 KO: $n=4$, one-way ANOVA, FDR $<0.05$, fold change is shown). $\log _{2}$ values for miRNAs are plotted. b) ISH hybridization analysis of the expression of miR449a in roof plate/CP at day E14.5 (http://www.eurexpress.org/ee/, (Diez-Roux et al., 2011)]). c) RT-qPCR analysis of miR-34/449 family members in brain ventricles from WT and TAp73 KO mice (WT: $n=3$; TAp73 KO: $n=4$. d) RT-qPCR analysis of miR449a in ED, FT, and trachea from WT and TAp73 KO mice (WT: ED, $n=3 ; \mathrm{FT}, n=7$; trachea, $n=4$; TAp73 KO: ED, $n=4 ; \mathrm{FT}, n=8$; trachea, $n=4)$. Cf. Figure 14a, c, and e for cutting area of ED, FT, and ventricle, respectively. Data are presented as the mean \pm SEM and relative to the WT group.

To identify the mechanism for miR449 upregulation upon TAp73 loss, we analyzed the p21$\mathrm{pRb} / \mathrm{E} 2 \mathrm{~F}$ pathway. Briefly, TAp73 induces the expression of cyclin-dependent kinase inhibitor 1 (P21, Cdkn1a) (Lee and La Thangue, 1999), thereby inhibiting cell cycle progression via the Rb/E2F pathway (Henley and Dick, 2012). As miR449 can be activated by E2F1 (Yang et al., 2009; Lizé et al., 2010b), miR449 upregulation in the absence of TAp73 might be due to the downregulation of P21 followed by an upregulation of E2F1. However, the expression levels of P21 (Cdkn1a) and its downstream effectors were not altered in the ventricles of TAp73 KO mice (Supplemental Figure 8a and b). Interestingly, transcript and protein expression levels of another E2F family member, namely E2F4, which is a potent inducer of multiciliogenesis (Stubbs et al., 2012; Boon et al., 2014; Ma et al., 2014; Kyrousi et al., 2015; Kyrousi et al., 2016; Kim et al., 2018), was markedly increased in TAp73 KO ventricles, despite only a mild increase of its cofactor Mcidas (Figure 24a and b). In contrast, E2F4 expression remained mostly unaltered in FTs and EDs, and downregulated in trachea upon TAp73 ablation (Supplemental Figure 7b - e). Therefore, increased E2F4 levels concurrent with a miR449 increase is restricted to the brain in TAp73 KO mice (Figure 27d).

E2F1 is capable of directly stimulating miR449 expression (Yang et al., 2009; Lizé et al., 2010b). Due to the conserved binding motif of E2F transcription factors, we hypothesized that the upregulation of E2F4 might contribute to the elevation of miR449 in the ventricles of TAp73 KO mice. Thus, we isolated the genomic region of miR449 ( $m i R 449$ is embedded in $C D C 20 B$ gene) containing putative E2F4 binding sites to assess the interaction between E2F4/MCIDAS and miR449 in a reporter-based assay (Table 3). Indeed, E2F4 in combination with MCIDAS elicited a strong transcriptional response from the miR449 locus, a reaction almost abolished by mutating a strong E2F consensus motif within the region (Figure 24c, Table 3). Altogether, these data indicate that the increased E2F4/MCIDAS activity stimulates miR449 expression in the ventricles of TAp73 KO mice, thereby creating a robust network ensuring multiciliogenesis in the brain upon TAp73 loss (Figure 27d). 
a

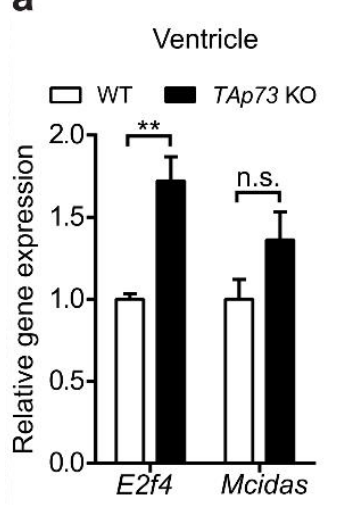

b

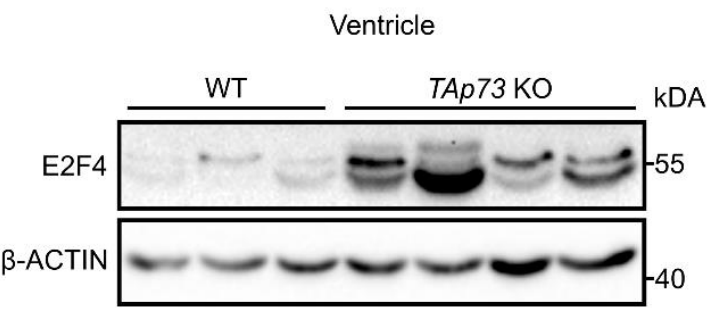

C

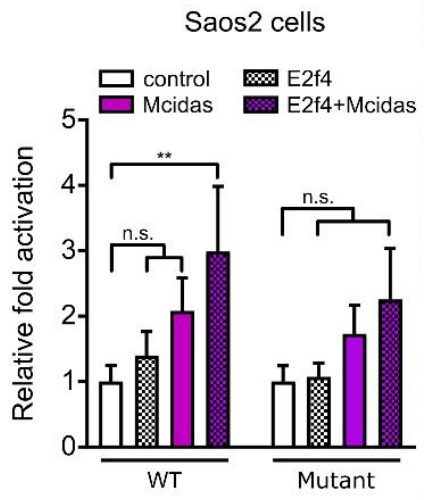

Figure 24: E2F4 levels are upregulated in the ventricles of the brain in TAp73 KO mice.

a) RT-qPCR analysis of E2f4/Mcidas in brain ventricles from WT and TAp73 KO mice (WT: $n=3 ;$ TAp73 KO: $n=4$. b) Immunoblot analysis of the expression of E2F4 in the ventricles of the brain from WT and TAp73 $\mathrm{KO}$ animals. $\beta$-ACTIN serves as a loading control. Cf. Figure $14 \mathrm{e}$ for cutting sites used for brain analysis. c) Luciferase assay of miR449 regulatory regions containing E2F binding motifs. Three consensus E2F binding sites in miR449 locus (http://jaspar.binf.ku.dk/) were placed in front of a luciferase cassette. A deletion mutant lacking the strongest consensus site was also created (Table 3). WT or mutant luciferase vector was cotransfected with empty vector (control), or vectors expressing E2f4, Mcidas, or both. Fold changes in luciferase activities relative to those of the control vector are shown $(n=5)$. Non-significant (n.s.). Data are presented as the mean \pm SEM.

\subsubsection{Combined deletion of TAp73 and miR449 impairs brain multiciliognesis}

Our data suggest that the crosstalk between E2F4 and miR449 partially compensates for the loss of TAp73 in brain multiciliogenesis. To address this, we generated mice with a deletion of the miR449 cluster in addition to TAp73. Strikingly, TAp73xmiR449 KO mice developed severe hydrocephalus (Figure 25a, Supplemental Figure 9a). Since defective ependymal and CP cilia contribute to the development of hydrocephalus (Ibanez-Tallon, 2004; Banizs et al., 2005; Banizs et al., 2007), we next assessed the ciliation in the ventricles of TAp73xmiR449 KO mice. Analysis of ARL13B expression revealed that, although miR449 loss alone resulted in a significant decrease in the number and length of cilia in CP epithelia, a more pronounced reduction in cilia was observed in TAp73xmiR449 $\mathrm{KO}$ mice (Figure 25b and c, Supplemental Figure 9b). Despite CP ciliary defects, the expression of $\mathrm{CP}$ epithelial differentiation markers including cytokeratins, AQP1, and Orthodenticle homeobox $\underline{2}$ (OTX2) was similar between WT, miR449 KO, and TAp73xmiR449 KO animals (Supplemental Figure 10a - c). Moreover, we observed mild defects in apical docking of BBs in ependymal cells in TAp73 KO and TAp73xmiR449 KO mice revealed by TEM (Figure 25d, Supplemental Figure 9d). In contrast, TAp73xmiR449 KO mice showed similar numbers of motile cilia on ependymal cells compared to WT mice (Supplemental Figure 9c). In agreement, ciliary beating of ependymal cilia and bead flow in cerebrospinal fluid were unaffected in TAp73xmiR449 KO mice (Supplemental Figure 9e). Therefore, additional loss of miR449 strongly impairs ciliogenesis in the $\mathrm{CP}$, but only slightly 
affects ependymal cilia, which is consistent with its prominent expression in the CP. Despite the role of Notch signaling in CP development and tumorigenesis (Bill et al., 2008; Li et al., 2016), RNAscope studies revealed similar expression of Notch pathway targets Hes1 and Hes5 in the roof plate of TAp73xmiR449 KO and miR449KO, compared to WT embryos at day E14.5 (Supplemental Figure 11a), indicating normal Notch pathway activity in both KO strains.

In summary, our study suggests that combined depletion of TAp73 and miR449 disrupts the robust multiciliogenesis program in the brain by impairing CP ciliogenesis, and thereby contributes to the development of hydrocephalus (Figure 27d and e).

We next analyzed other multiciliated tissues in TAp73xmiR449 KO mice to evaluate whether miR449 loss intensifies the ciliary defects already present upon TAp73 deficiency. Immunofluorescent staining revealed a significant reduction in the expression of Ac- $\alpha-T U B$ in trachea of TAp73xmiR449 KO compared to WT animals (Figure 26a and b). Consistently, ultrastructural studies showed that BB were not properly docked to the apical cell surface and consequently less cilia were formed in TAp73xmiR449 KO mice (Figure 26c, Supplemental Figure 12a). However, the deletion of the miR449 cluster failed to exacerbate multiciliogenesis defects in the absence of TAp73 as the observed ciliary defect was similar to the one we previously described in TAp73 KO mice (Nemajerova et al., 2016). Similarly, immunostainings for the cilia marker Ac- $\alpha$-TUB revealed that additional loss of miR449 did not further enhance cilia reduction in EDs compared to TAp73 KO mice (Supplemental Figure $12 \mathrm{~b}$ and c), despite a strong upregulation of miR449 in TAp73 KO EDs (Figure 23d).

To conclude, our data indicate that TAp73 utilizes the unique topology of its transcriptional network to communicate with the miR-34/449 family members and other crucial regulators of multiciliogenesis e.g. E2F4/MCIDAS to regulate brain multiciliogenesis (Figure 27d and e). This contrasts with the reproductive ducts, where loss of TAp73 severely impairs multiciliogenesis (Figure 27b and c). 
a
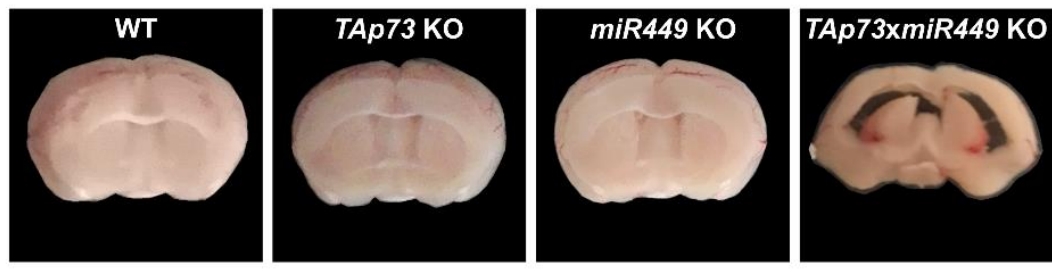

b

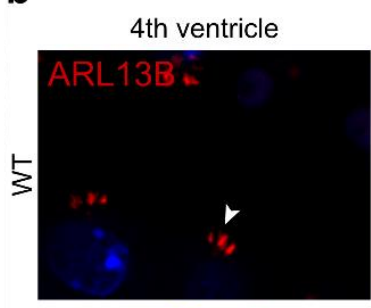

Lateral ventricle

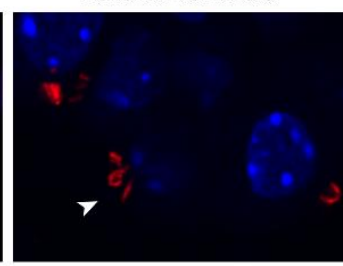

C
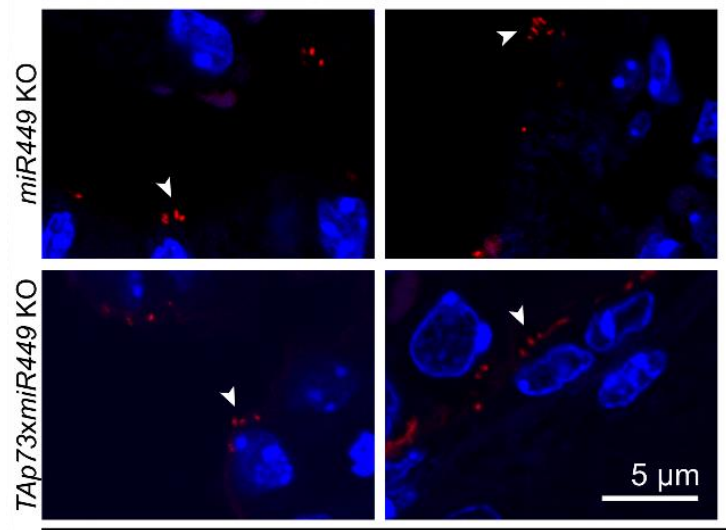

4th ventricle

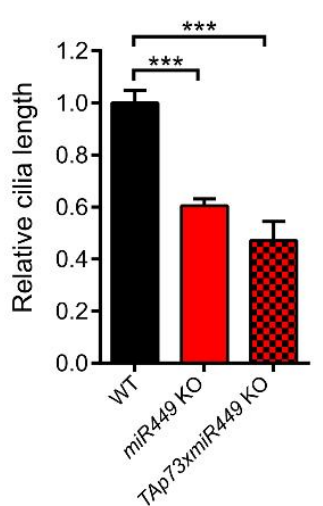

Lateral ventricle

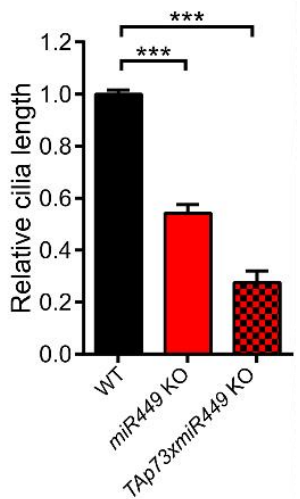

Choroid plexus

d

WT

TAp73 KO

TAp73xmiR449 KO

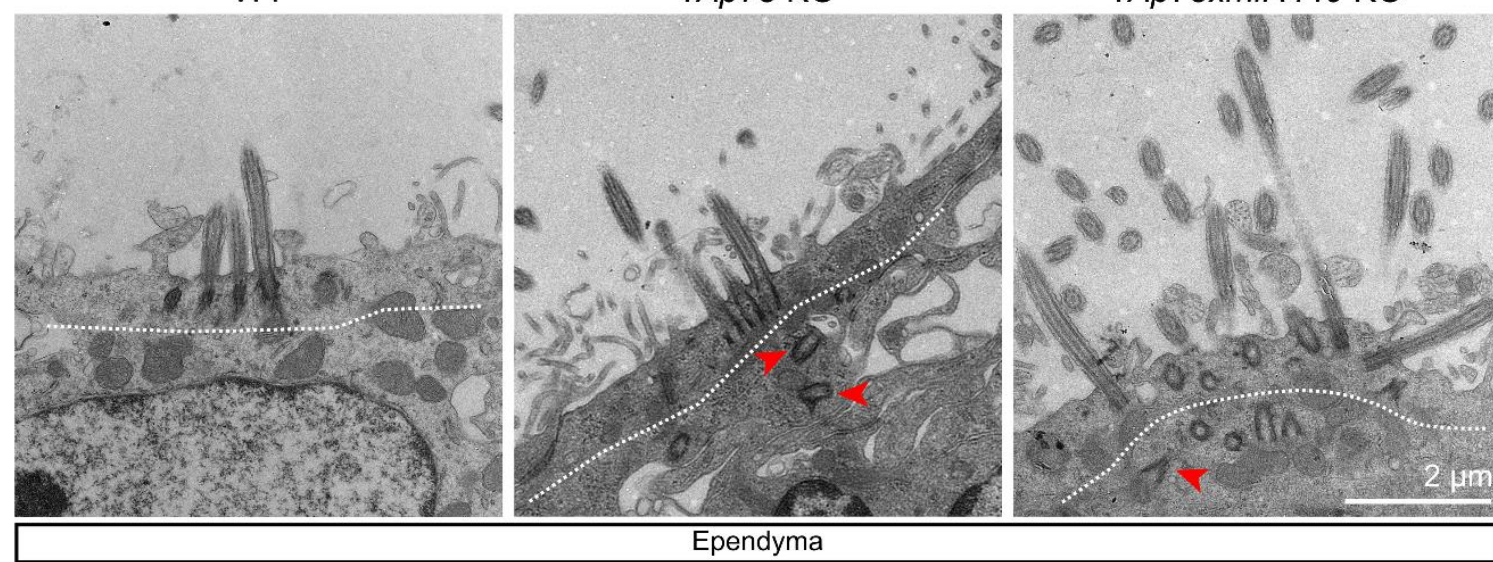

Figure 25: TAp73 functions through miR-34/449 family in brain multiciliogenesis.

a) Coronal brain slices from WT (40 weeks), TAp73 KO (12 weeks), miR449 KO (7 weeks), and TAp73xmiR449 KO, 12 weeks) mice. Notice the enlarged lateral ventricles in TAp73xmiR449 KO mice. b) Expression of ARL13B (red) in CP epithelial cells of the 4th and lateral ventricle in WT, miR449 KO, and TAp73xmiR449 KO animals. DAPI staining (blue) labels nuclei. c) Quantitation of cilia length of CP epithelial cells (4th ventricle: WT: $n=4$ cells from 2 mice; miR449 KO: $n=18$ cells from 4 mice; TAp73xmiR449 KO: $n=8$ cells from 3 mice Lateral ventricle: WT: $n=4$ cells from 2 mice; miR449 KO: $n=14$ cells from 4 mice; TAp73xmiR449 KO: $n=8$ cells from 3 mice). Data are presented as the mean \pm SEM and relative to the WT group. d) Representative photomicrographs of TEM of multiciliated ependymal cells in WT, TAp73 KO, and TAp73xmiR449 KO mice. Notice the presence of mislocated BBs in TAp73 KO and TAp73xmiR449 KO mice (arrows). 


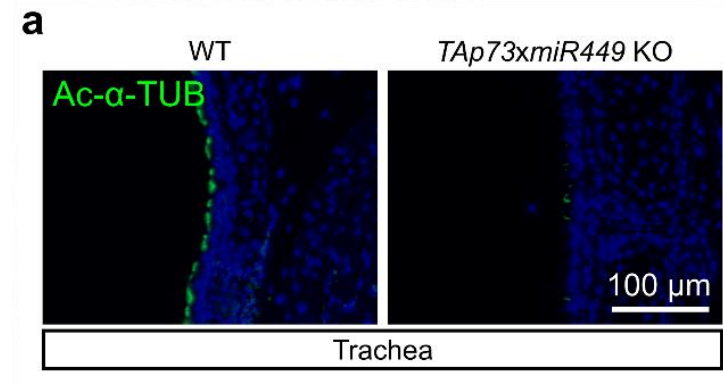

b

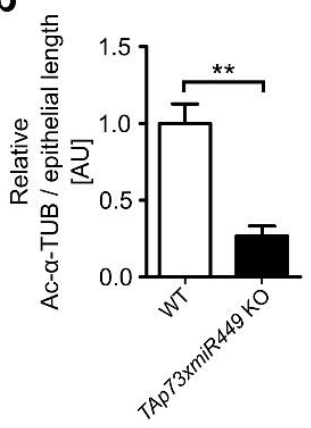

C

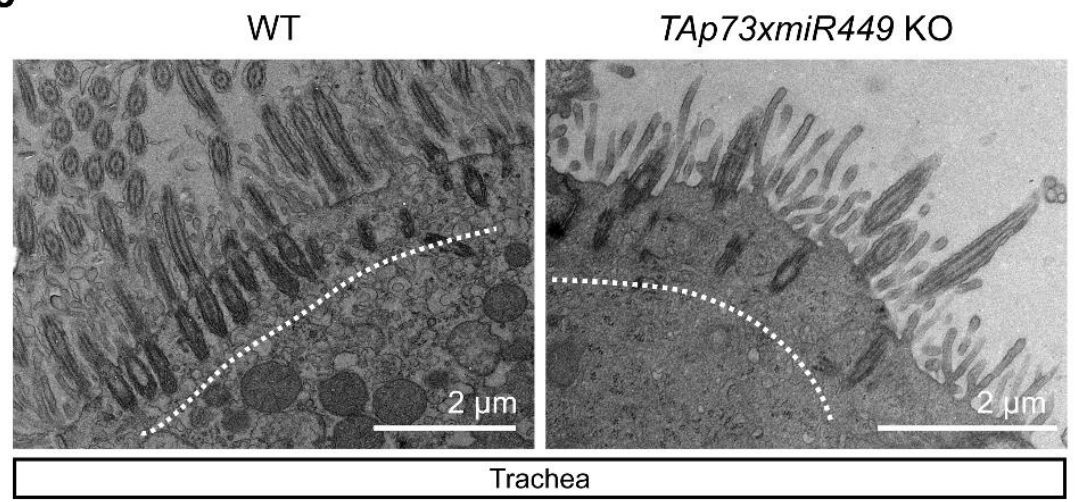

Figure 26: $m i R 449$ cluster fails to exacerbate airway multiciliogenesis defects in the absence of TAp73.

a) Expression of Ac-a-TUB (green) in trachea of WT and TAp73xmiR449 KO mice. DAPI staining (blue) labels nuclei. b) Quantitation of Ac-a-TUB signal normalized to epithelial length ( $n=4$ samples/genotype). Data are presented as the mean \pm SEM and relative to WT group. c) Representative TEM images of trachea of WT and TAp73xmiR449 KO mice. 
a

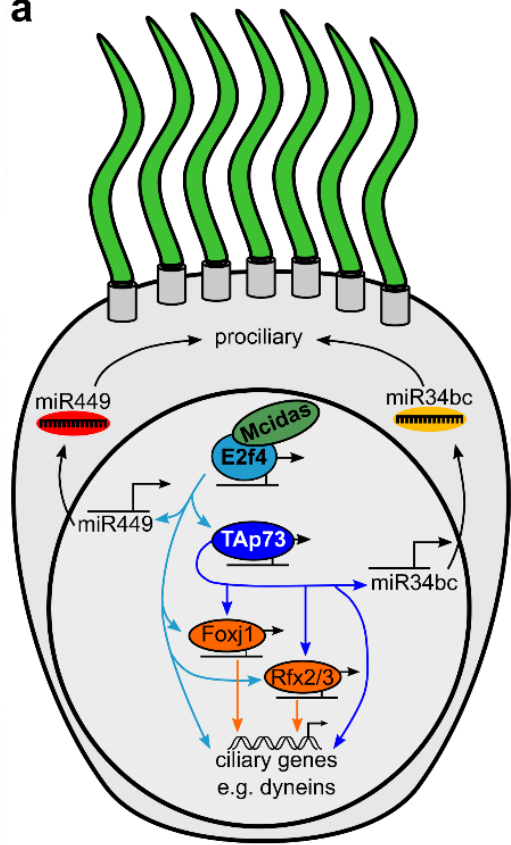

Wild type b

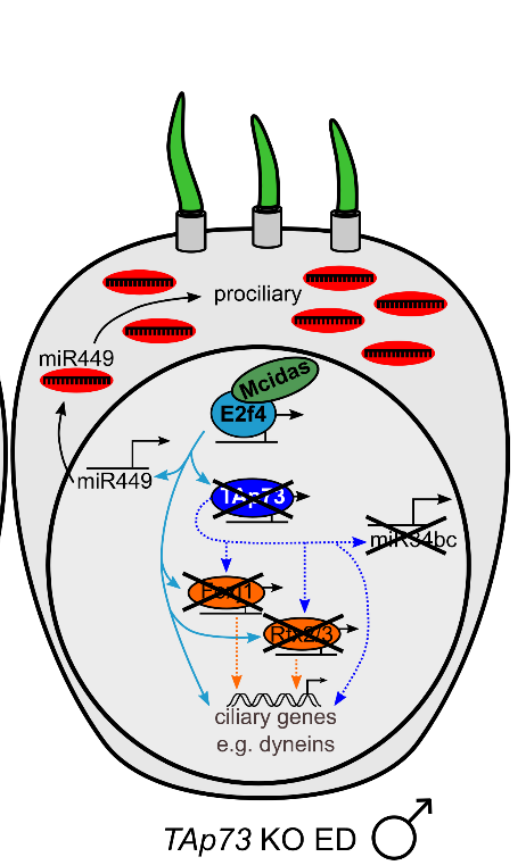

C

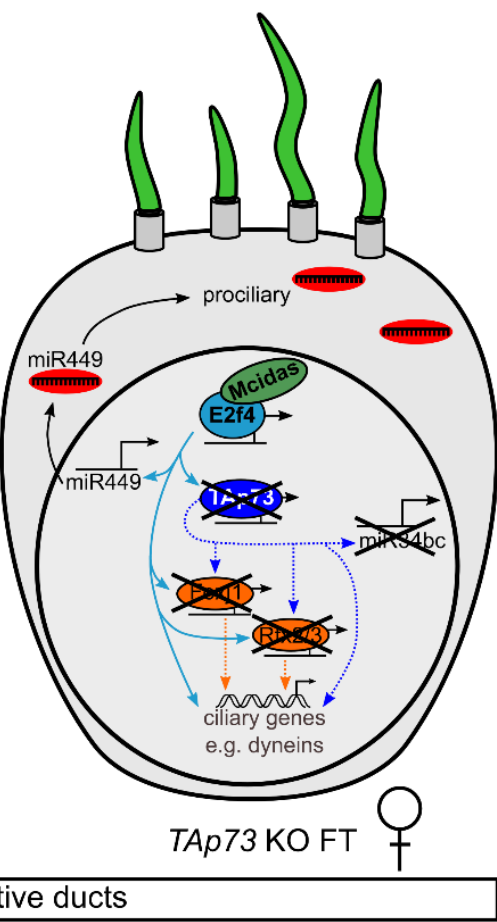

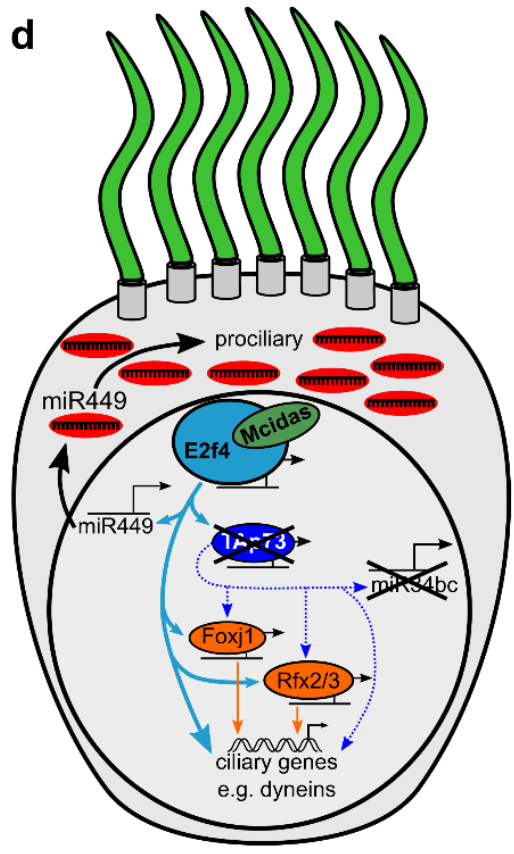

TAp73 KO

e

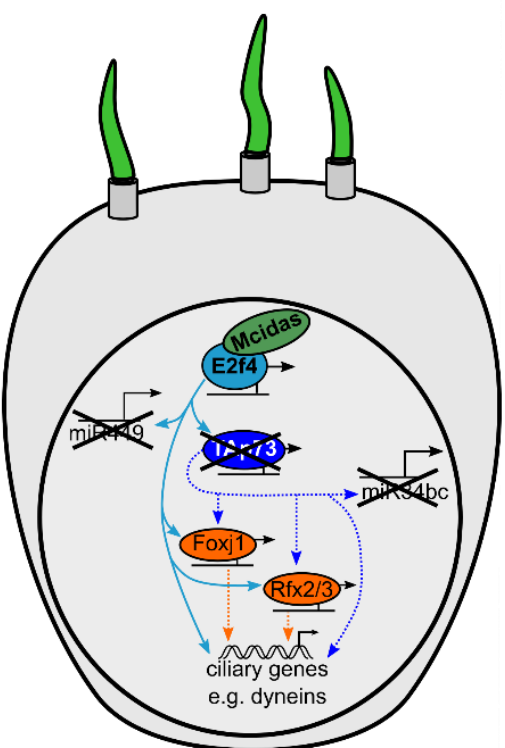

TAp73xmiR449 KO

Brain ventricles/Choroid plexus

Figure 27: Schematic diagram of the molecular mechanisms of TAp73-driven multiciliogenesis in diverse tissues.

a) TAp73-dependent transcriptional network, including dyneins, miR34b,c, Foxj1, Rfx2, and Rfx3 factors, critically regulates multiciliogenesis in various ciliated tissues downstream of E2f4/Mcidas. $b$ and c) In reproductive ducts, TAp73 ensures the generation of MCCs and proper gamete and zygote transport, whereas loss of TAp73 impairs fertility in male (b) and female (c) mice. d) TAp73 is not essential for multiciliogenesis in the brain. However, TAp73 loss leads to upregulation of pro-ciliogenic E2F4 and its target miR449. e) Removal of miR449 in addition to TAp73 loss leads to reduced motile cilia formation in the CP epithelium and severe hydrocephalus, indicating that miR449 partially compensates loss of TAp73 in brain ciliogenesis. 


\subsubsection{Supplemental Figures}

a WT

TAp73 KO
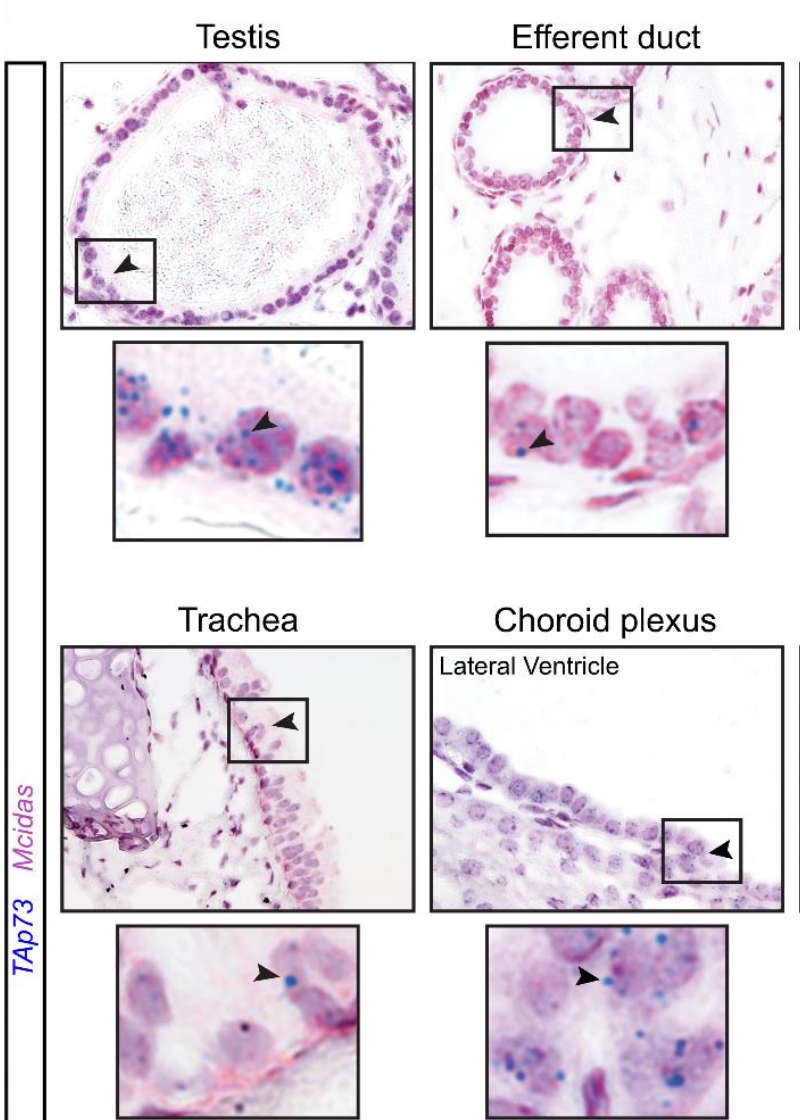

Fallopian tube
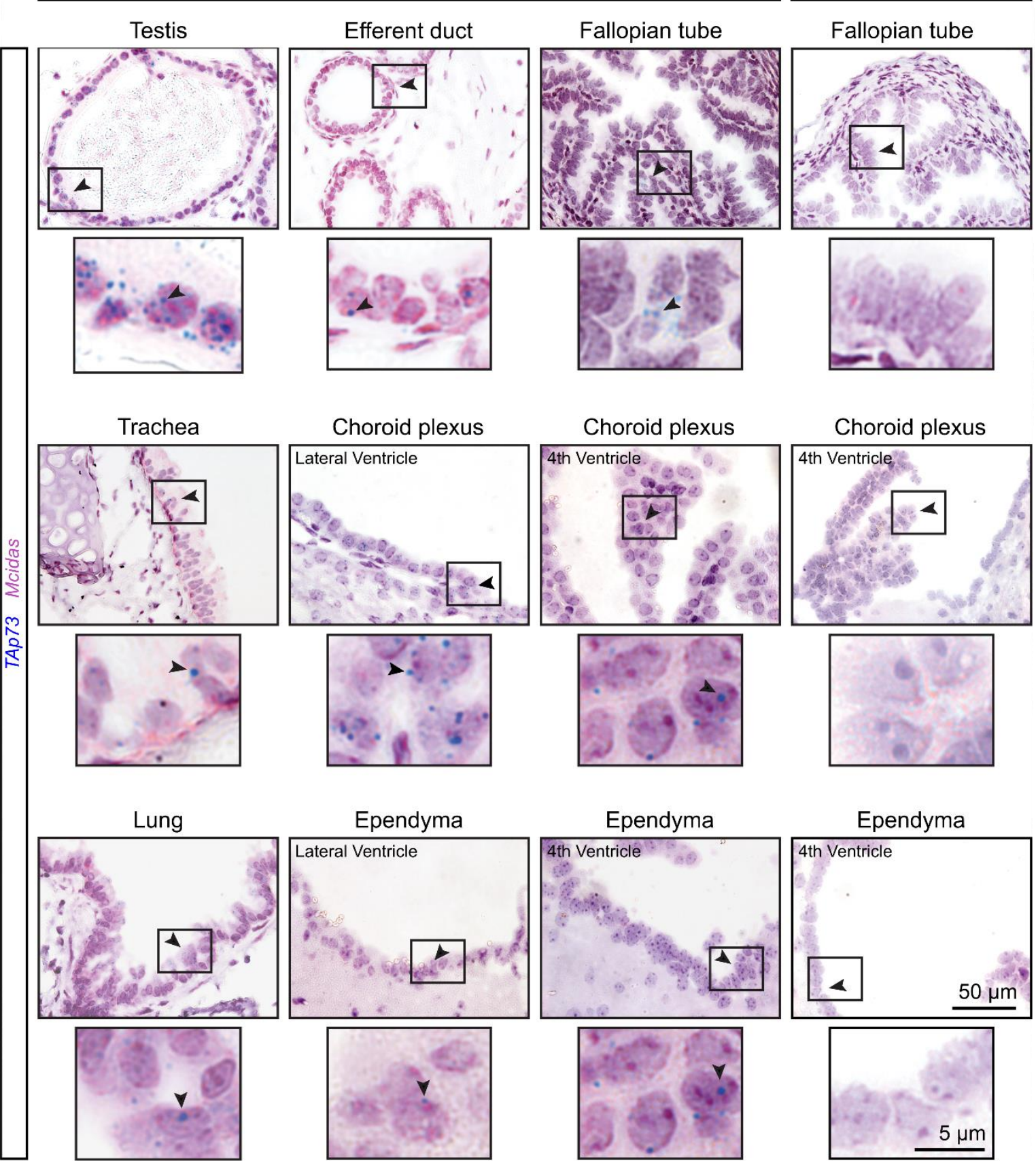

\section{Supplemental Figure 1: Expression of TAp73 in multiciliated tissues.}

a) RNAscope analysis of TAp73 (blue) and Mcidas expression (red) in testis, efferent duct, fallopian tube, FT, trachea, lung, ependymal and choroid plexus cells in lateral and 4th ventricle in adult mice. Boxed regions are magnified below each image and show TAp73-positive cells. In contrast to WT cells, TAp73 mRNA is absent in TAp73 KO cells. 


\section{a}

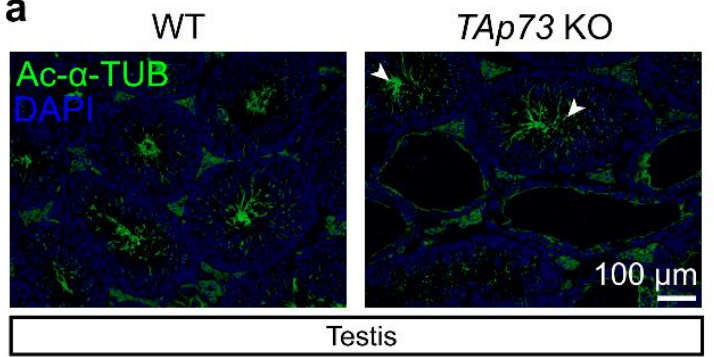

C

WT

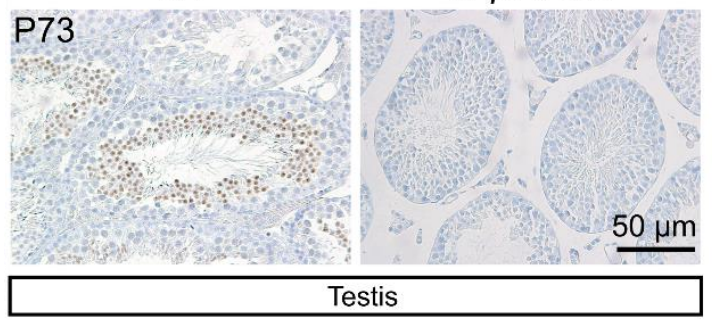

Testis b
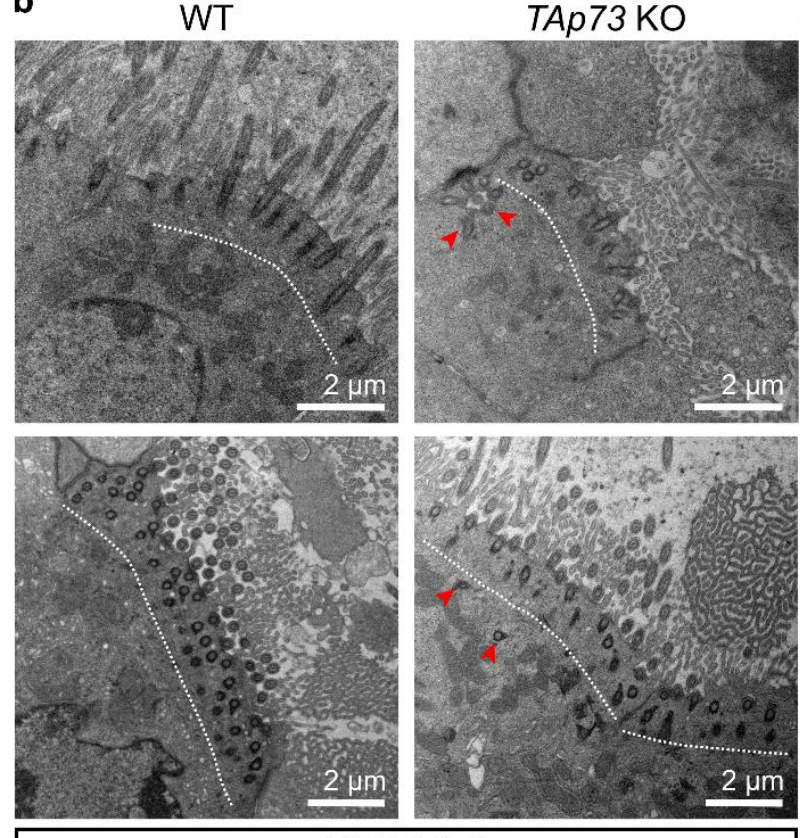

Efferent duct

d Testis

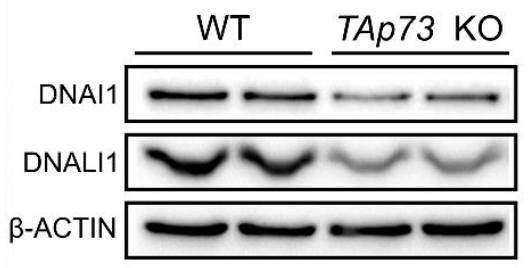

Supplemental Figure 2: Loss of TAp73 impairs multiciliogenesis in the male reproductive duct. a) Representative images of the expression of Ac-a-TUB (green) are shown in testis of WT and TAp73 KO mice. DAPI staining (blue) labels nuclei. Notice the presence of spermatozoa (Ac-a-TUB') in TAp73 KO testis (arrowheads). b) TEM of MCCs in EDs of WT and TAp73 KO mice. Red arrowheads indicate mislocalization of BBs. c) Expression of P73 in testis of WT and TAp73 KO mice. Notice that P73-positive cells are absent in TAp73 KO testis. d) Immunoblot analysis of the expression of DNAl1 and DNALI1 in the testis from WT and TAp73 KO animals. $\beta$-ACTIN serves as a loading control. Notice that DNAI1 and DNALI1 levels are reduced in TAp73 mutant testis compared to WT animals. 
a
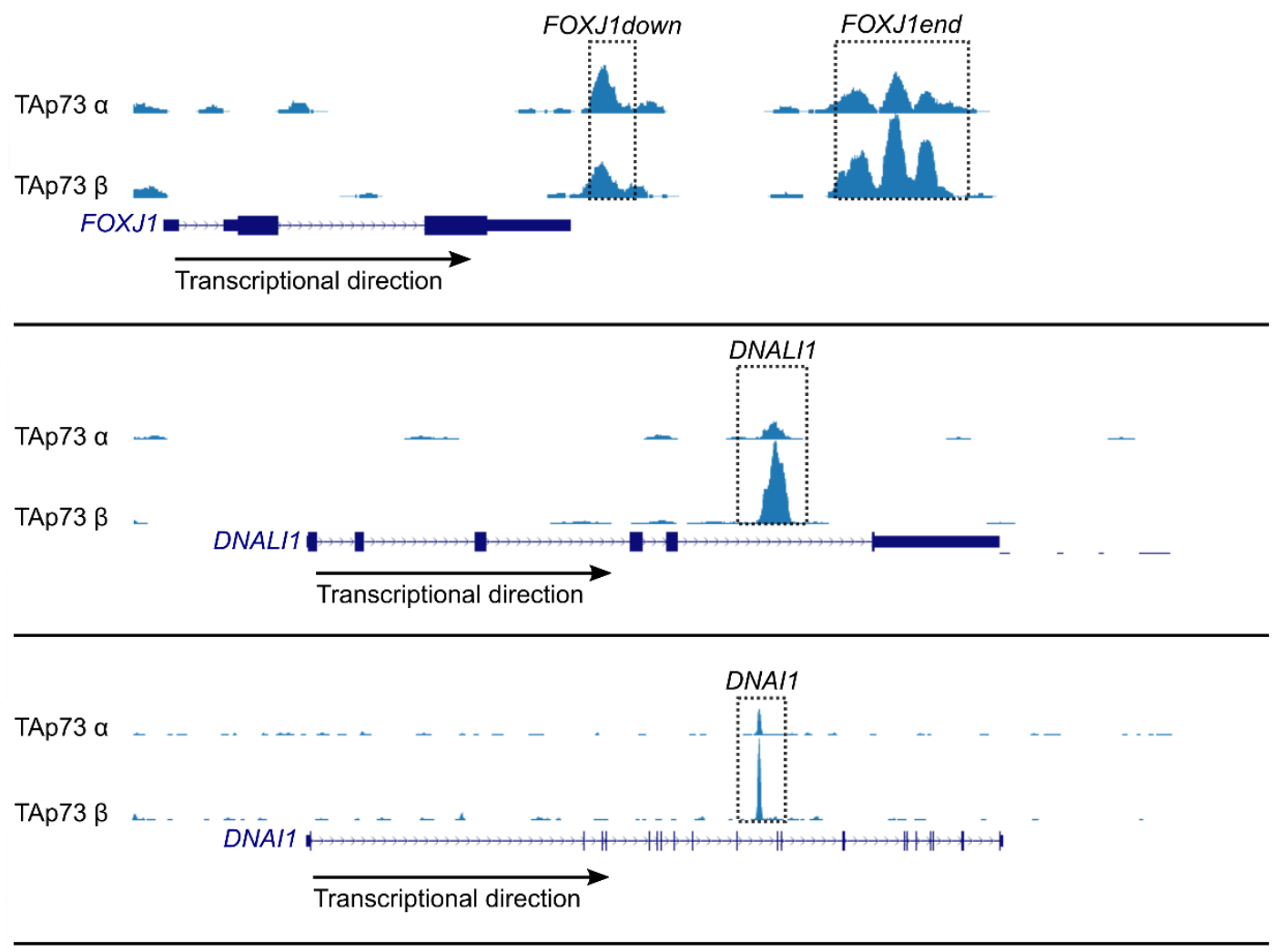

Supplemental Figure 3: Binding of TAp73 is enriched at genomic loci from ciliary genes.

a) TAp73 binding at FOXJ1, DNALI1, and DNAl1 genomic loci is shown in results from ChIP-seq (Koeppel et al., 2011), Geo accession no. GSE15780. Boxed regions mark genomic loci enriched with TAp73 binding and validated by ChIP-qPCR (Figure 17a). 
a

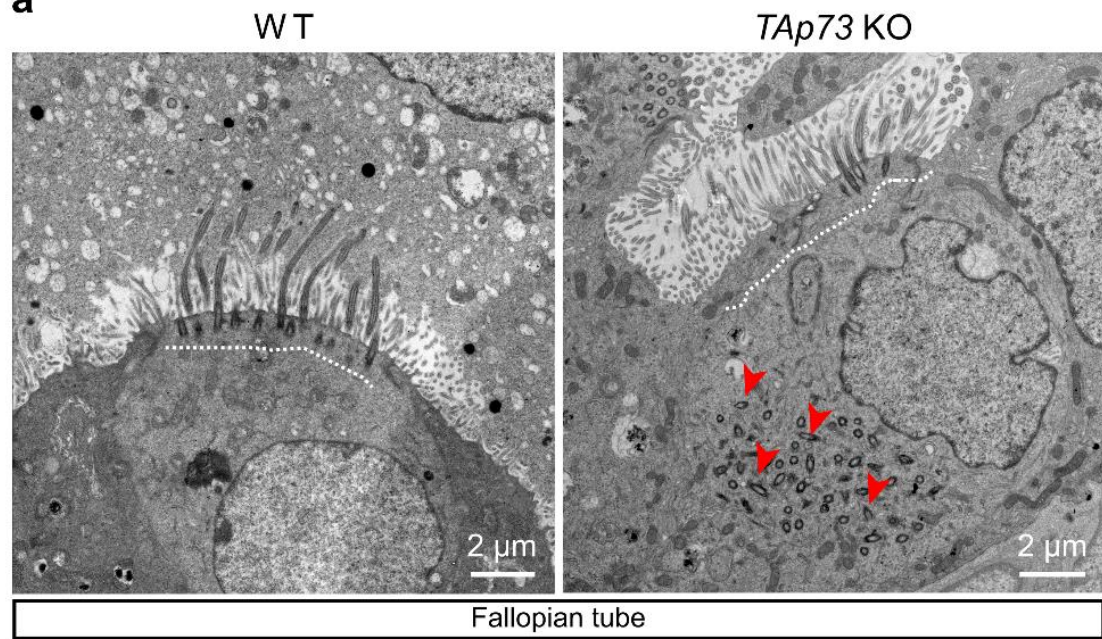

b

DNALI1

FOXJ1
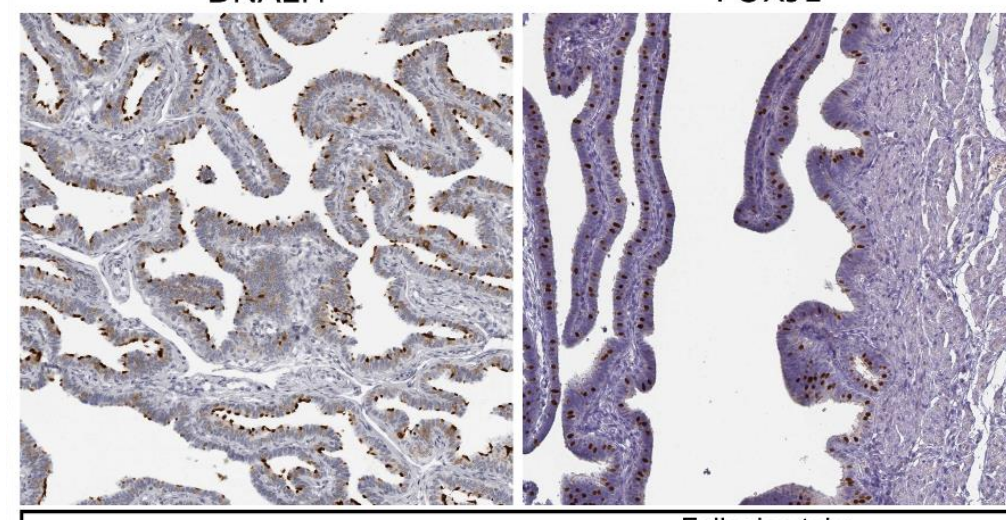

DNAI1

Fallopian tube

\section{Supplemental Figure 4: TAp73 KO mice show defective motile cilia in fallopian tubes.}

a) TEM of MCCs in FTs from WT and TAp73 KO mice. Red arrowheads indicate mislocalization of BBs. b) Human FT epithelia express DNALI1, FOXJ1, and DNAI1. Images were retrieved from the Human Protein Atlas:

DNALI1: http://www.proteinatlas.org/ENSG00000163879-DNALI1/tissue/fallopian+tube, FOXJ1: http://www.proteinatlas.org/ENSG00000129654-FOXJ1/tissue/fallopian+tube, DNAl1: http://www.proteinatlas.org/ENSG00000122735-DNAl1/tissue/fallopian+tube. 
a
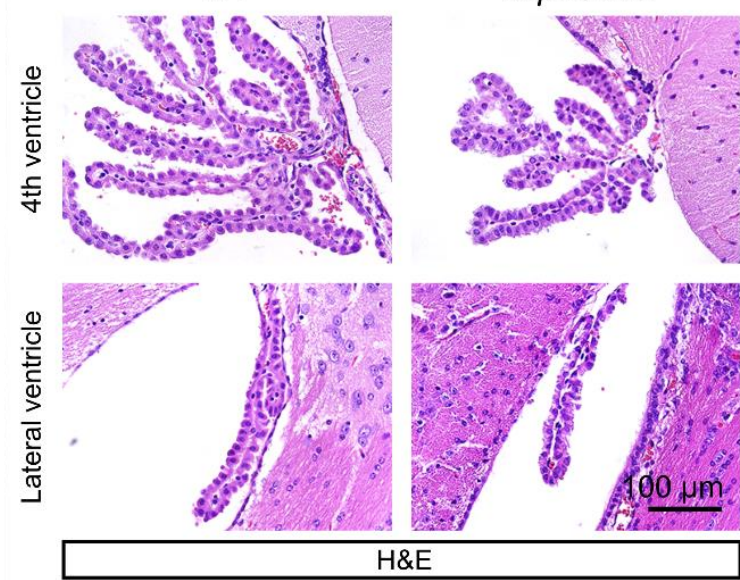

C
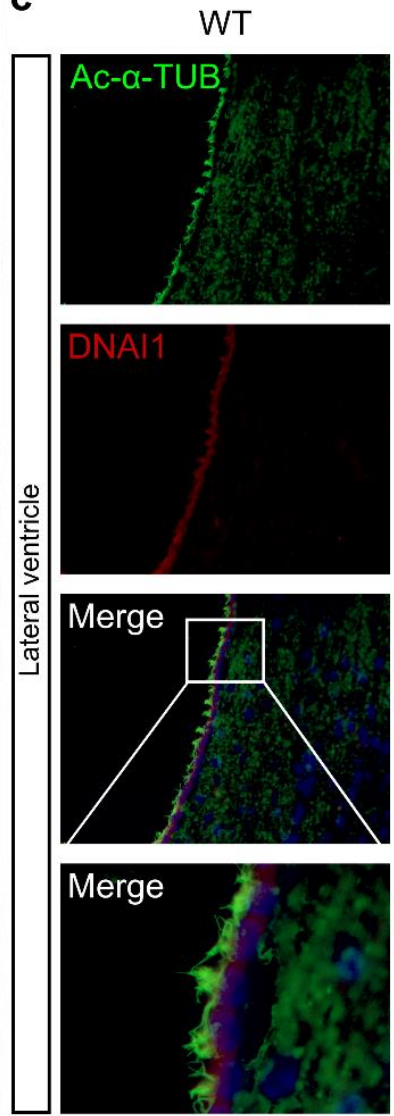

TAp73 KO
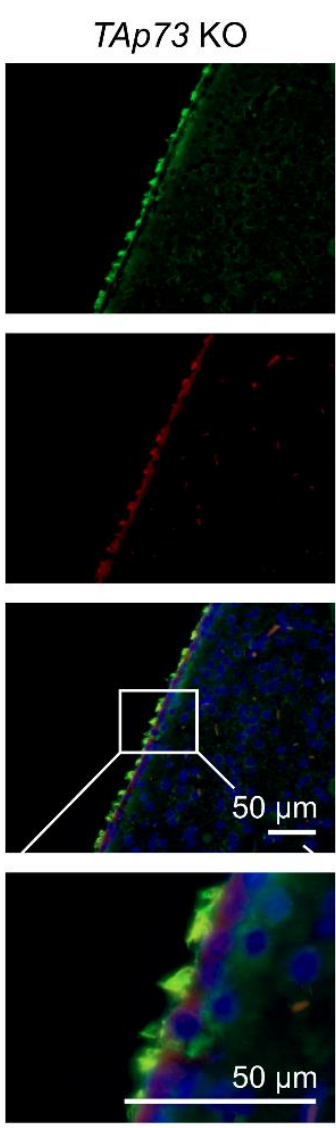

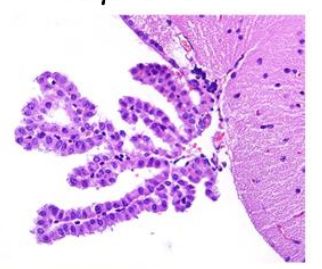

b
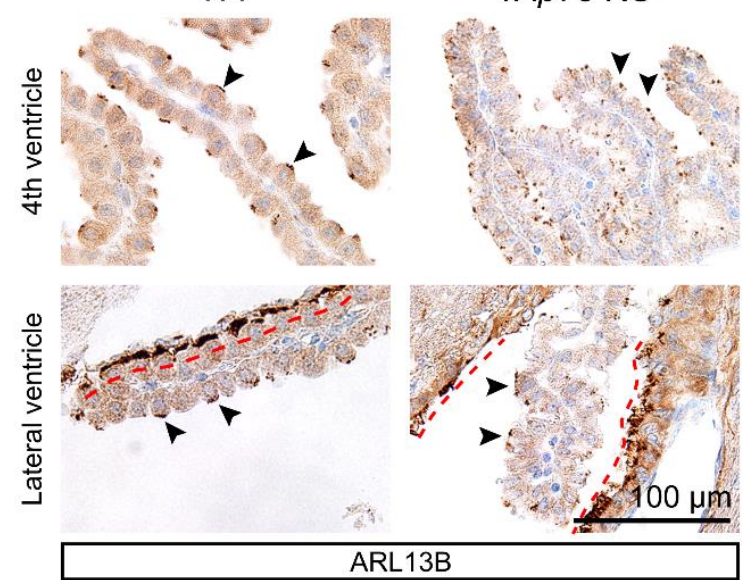

d

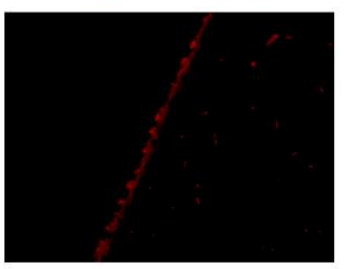

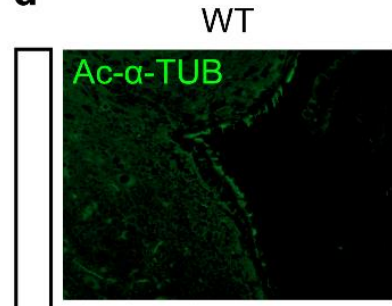
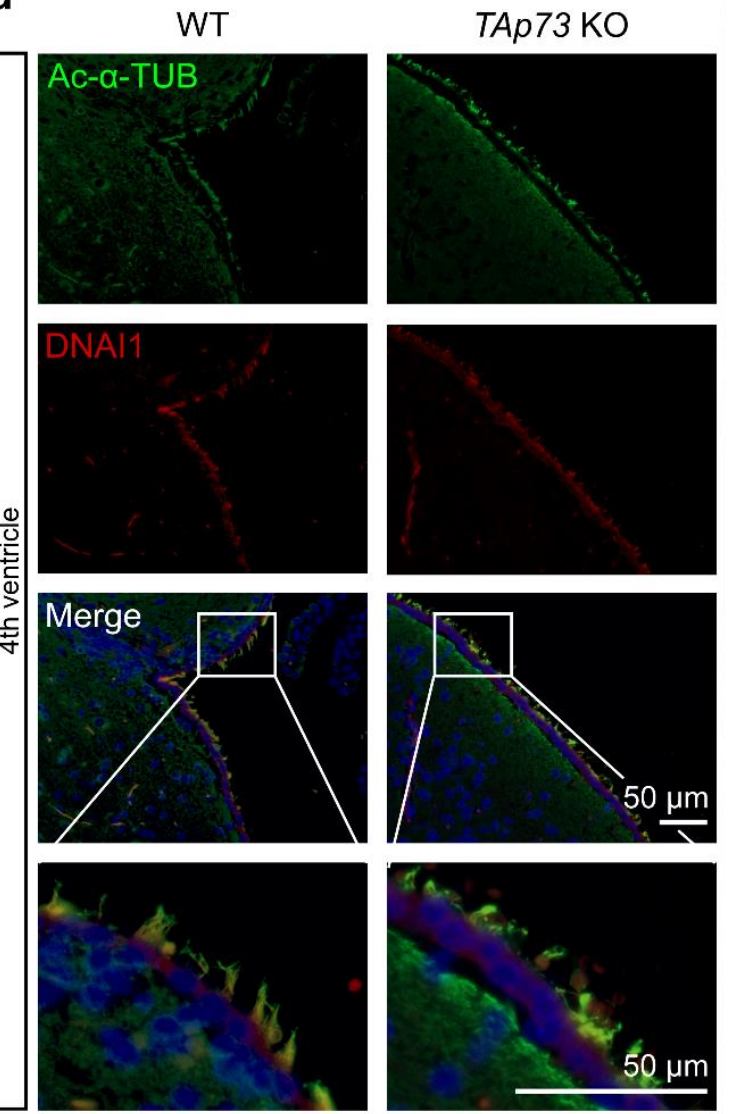

TAp73 KO

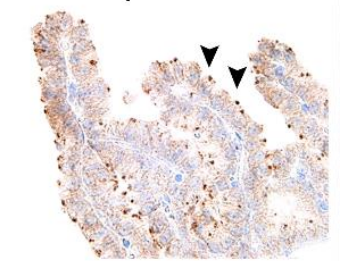

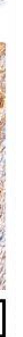



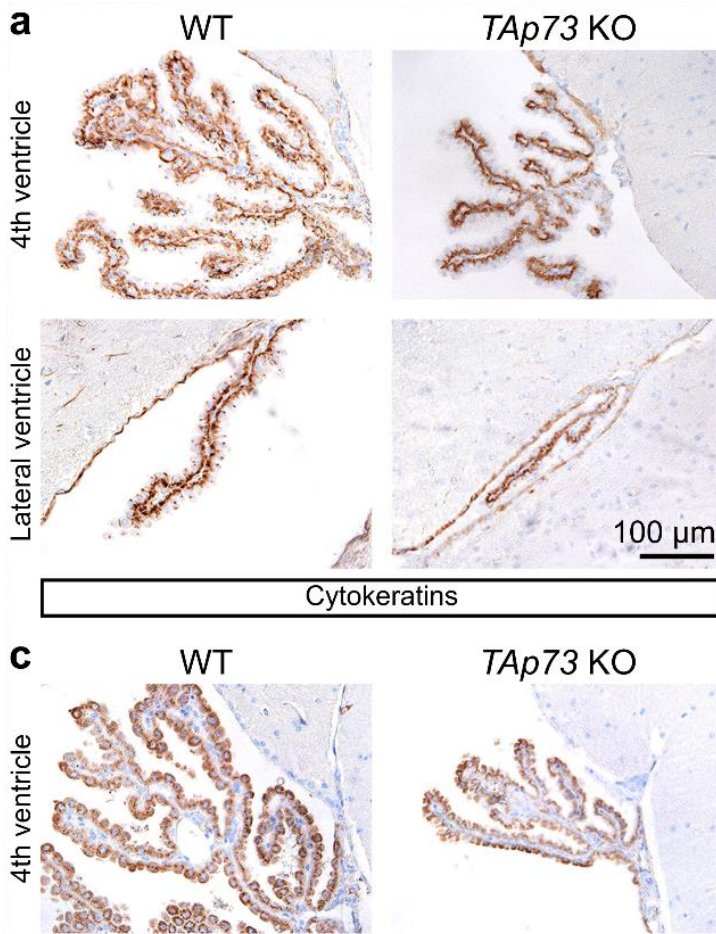

TAp73 KO

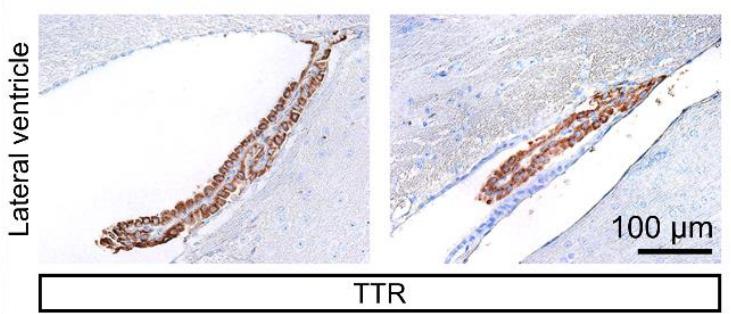

b

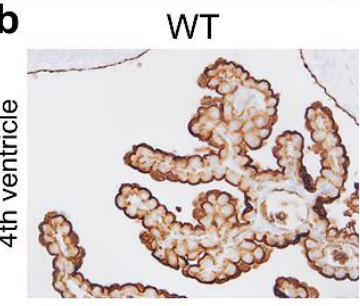

TAp73 KO

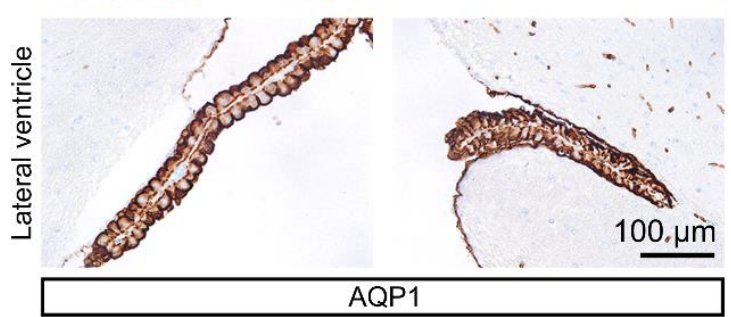

d

WT

TAp73 KO
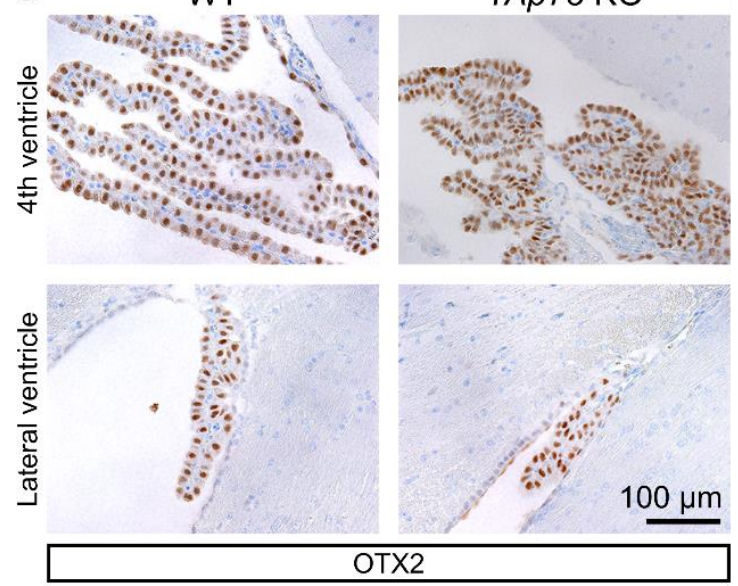

Supplemental Figure 6: Epithelial differentiation of CP cells is not affected upon TAp73 loss.

a - d) Representative images of the expression of Cytokeratins (a), AQP1 (b), Transthyretin (TTR, c), and Orthodenticle homeobox $\underline{2}$ (OTX2, d) are shown in CP epithelium of the 4th and lateral ventricles of WT and $\bar{T} A$ 73 KO animals. 
a

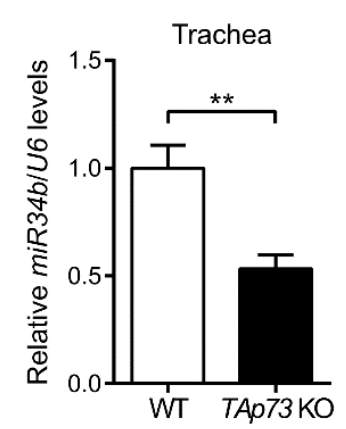

C
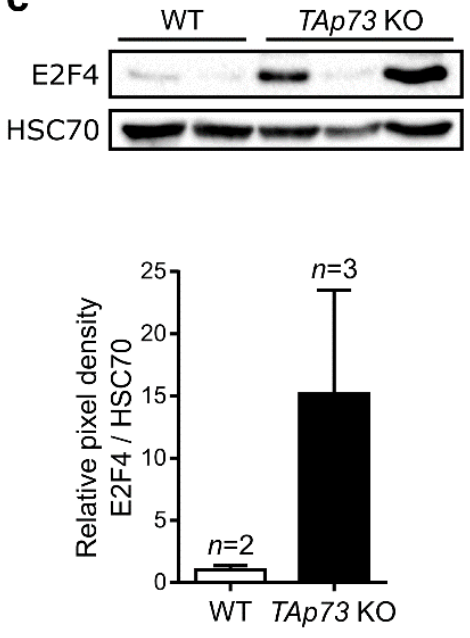

b
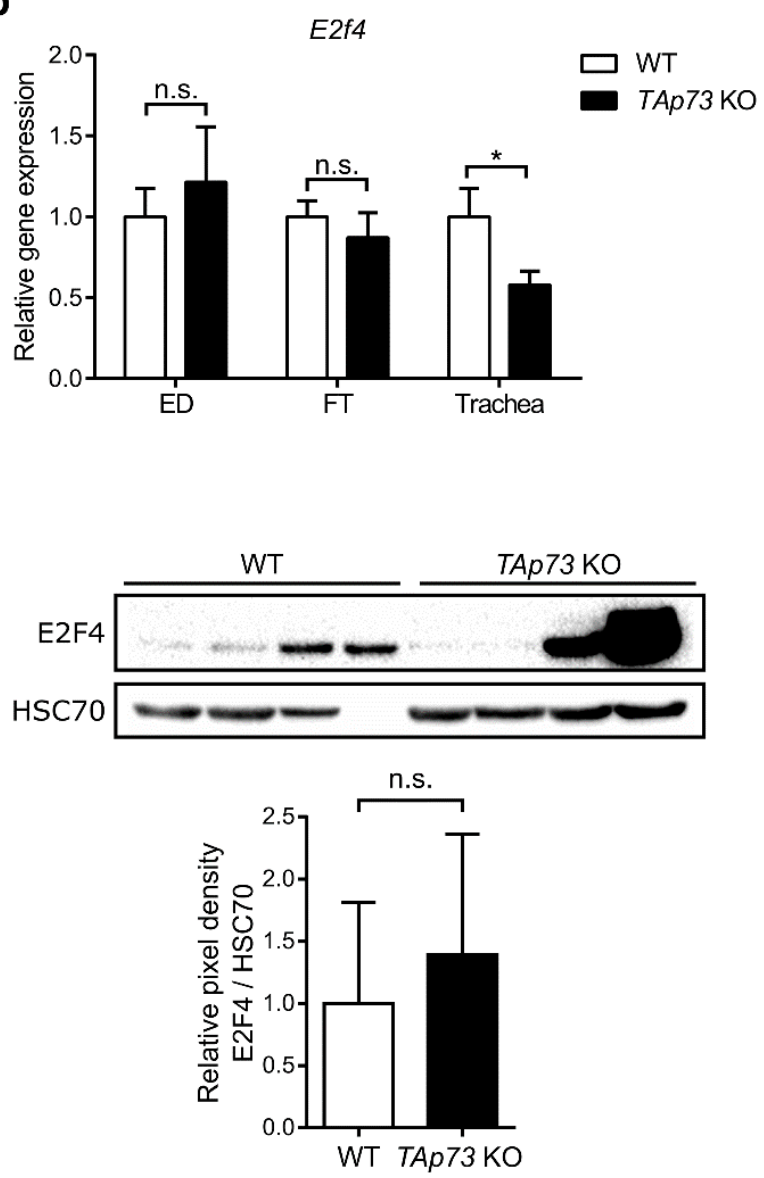

Fallopian tube

d

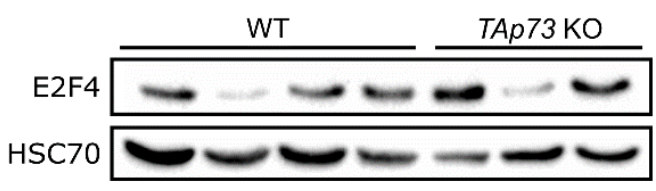

e
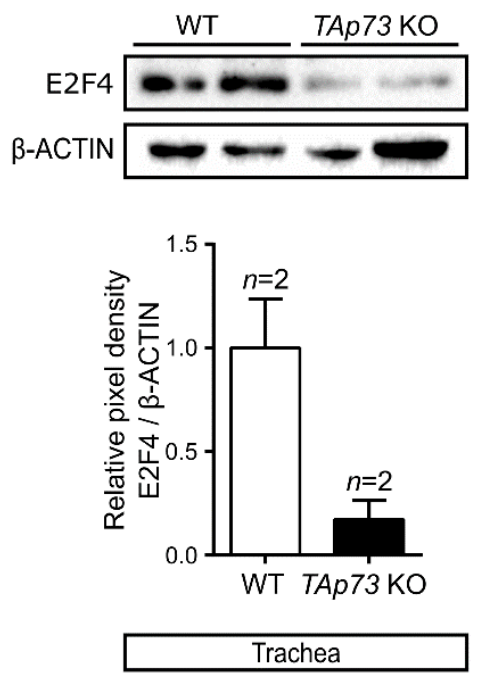

Supplemental Figure 7: Expression analysis of pro-ciliogenic factors in multiciliated tissues of TAp73 KO mice.

a) RT-qPCR analysis of miR34b expression in trachea from WT and TAp73 KO mice (WT: $n=3$; TAp73 KO: $n=4)$. b) RT-qPCR analysis of E2f4 expression in ED, FT, and trachea from WT and TAp73 KO mice (WT: $\mathrm{ED}, n=5 ; \mathrm{FT}, n=7$; trachea, $n=4$; TAp73 KO: ED, $n=4 ; \mathrm{FT}, n=8$; trachea, $n=4)$. c - e) Immunoblot analysis of the expression of E2F4 in FT (c), ED (d), and trachea (e) in WT and TAp73 KO animals. B-ACTIN or HSC70 serve as a loading control. Quantitation of the signal intensity of E2F4 bands normalized to that of the loading ctrl is shown below each immunoblot ( $n=$ number of bands per genotype). Cf. Figure 14a and $c$ for cutting area of ED and FT, respectively. Non-significant (n.s.). Data are presented as the mean \pm SEM and relative to the WT group. 
a

Ventricles

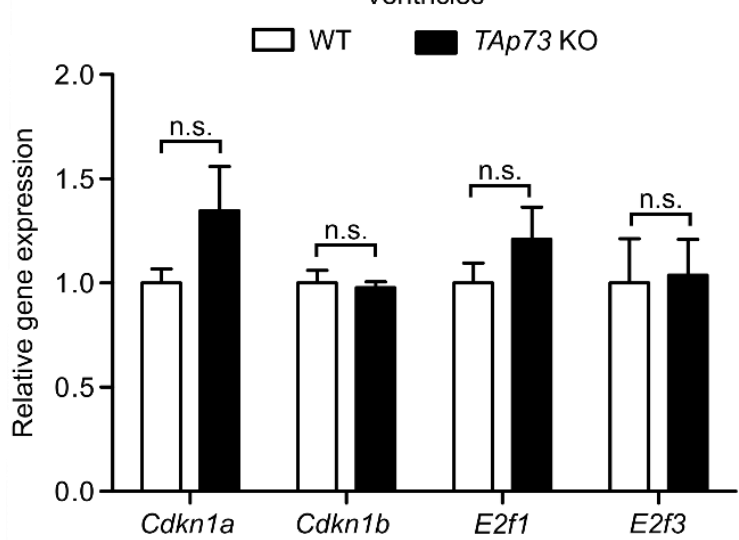

b

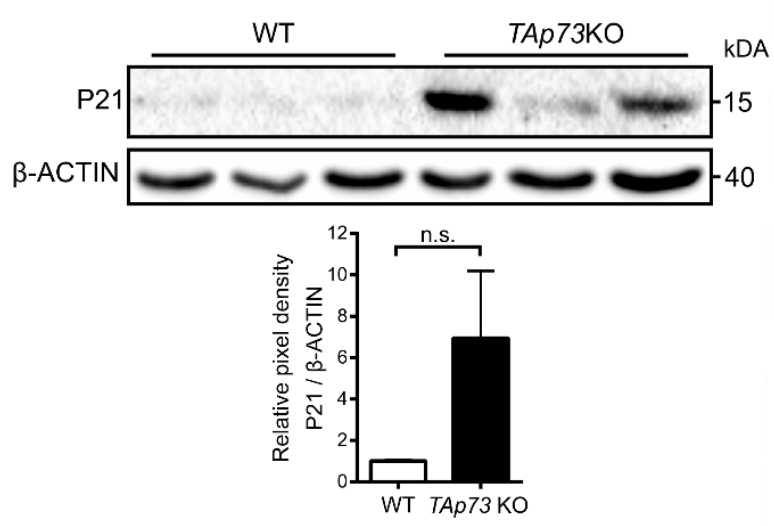

Supplemental Figure 8: $\quad \mathrm{pRb} / \mathrm{E} 2 \mathrm{~F}$ pathway activity is not deregulated in $T A p 73 \mathrm{KO}$ ventricles.

a) RT-qPCR analysis of Cdkn1a (P21), Cdkn1b, E2f1, and E2f3 in the ventricles of WT and TAp73 KO animals $(n=4)$. b) Immunoblot analysis of P21 expression in the ventricles of WT and TAp73 KO animals. Graph shows quantification of the signal intensity of P21 bands relative to that of $\beta$-ACTIN $(n=3)$. Nonsignificant (n.s.). Data are presented as the mean \pm SEM. and relative to the WT group. 
a

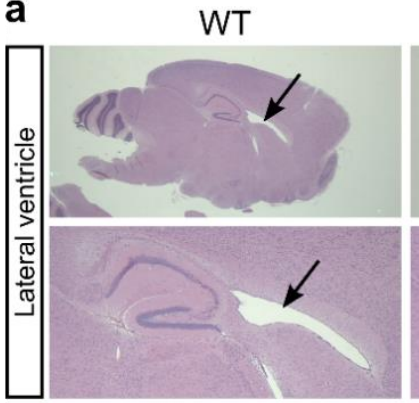

TAp73 KO

$\operatorname{miR} 449 \mathrm{KO}$

TAp73xmiR449 KO
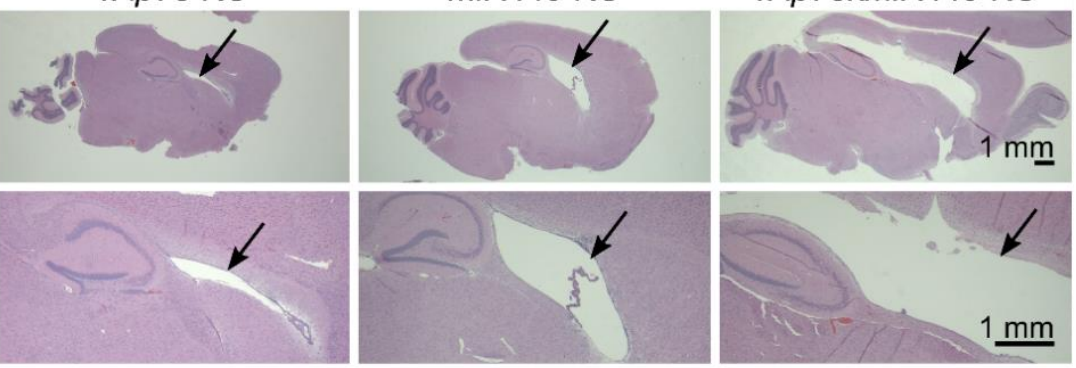

b

Lateral ventricle

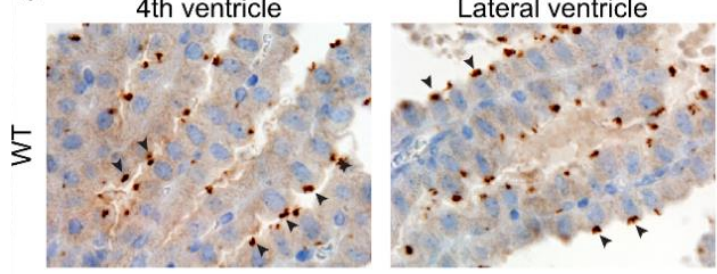

C
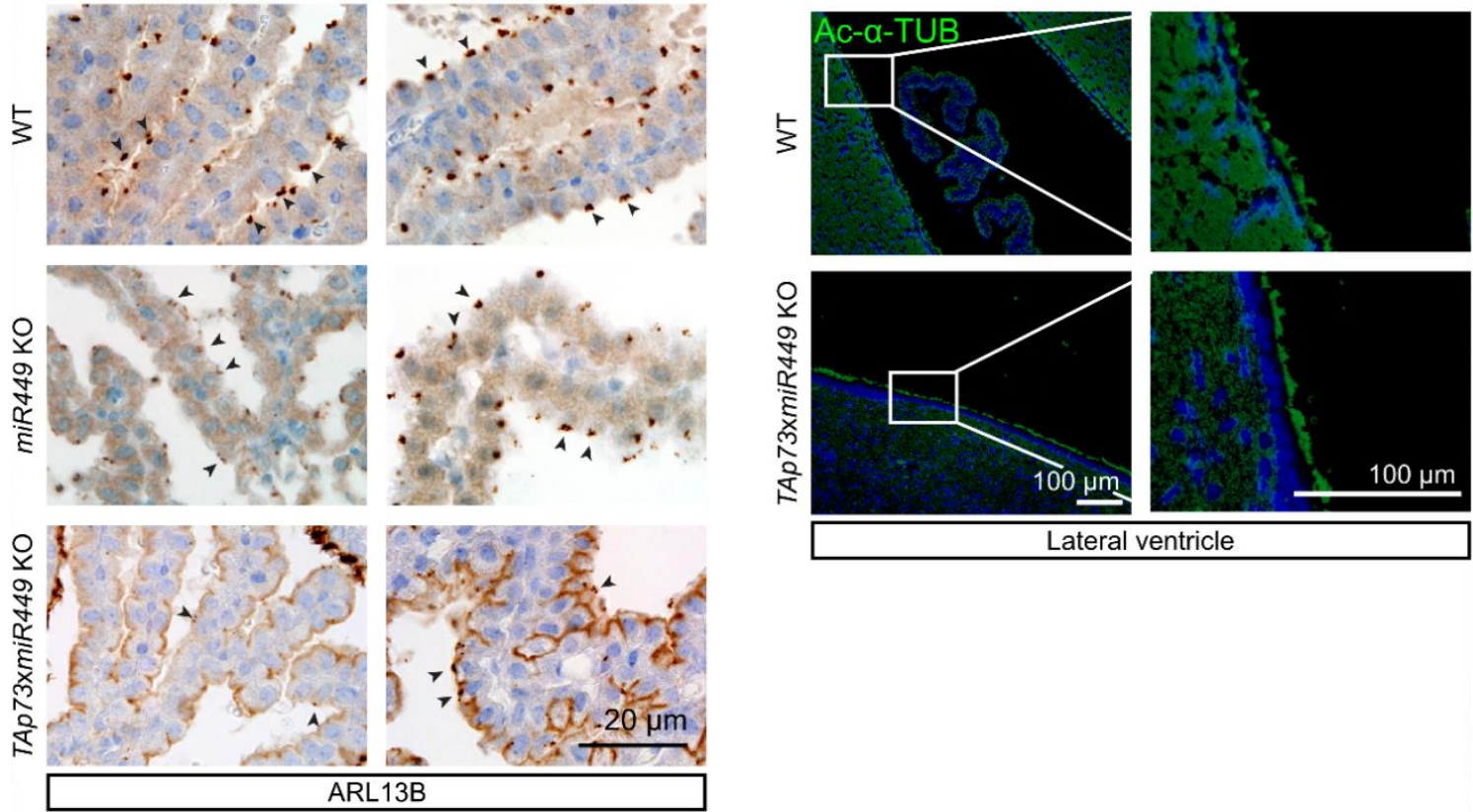

Lateral ventricle
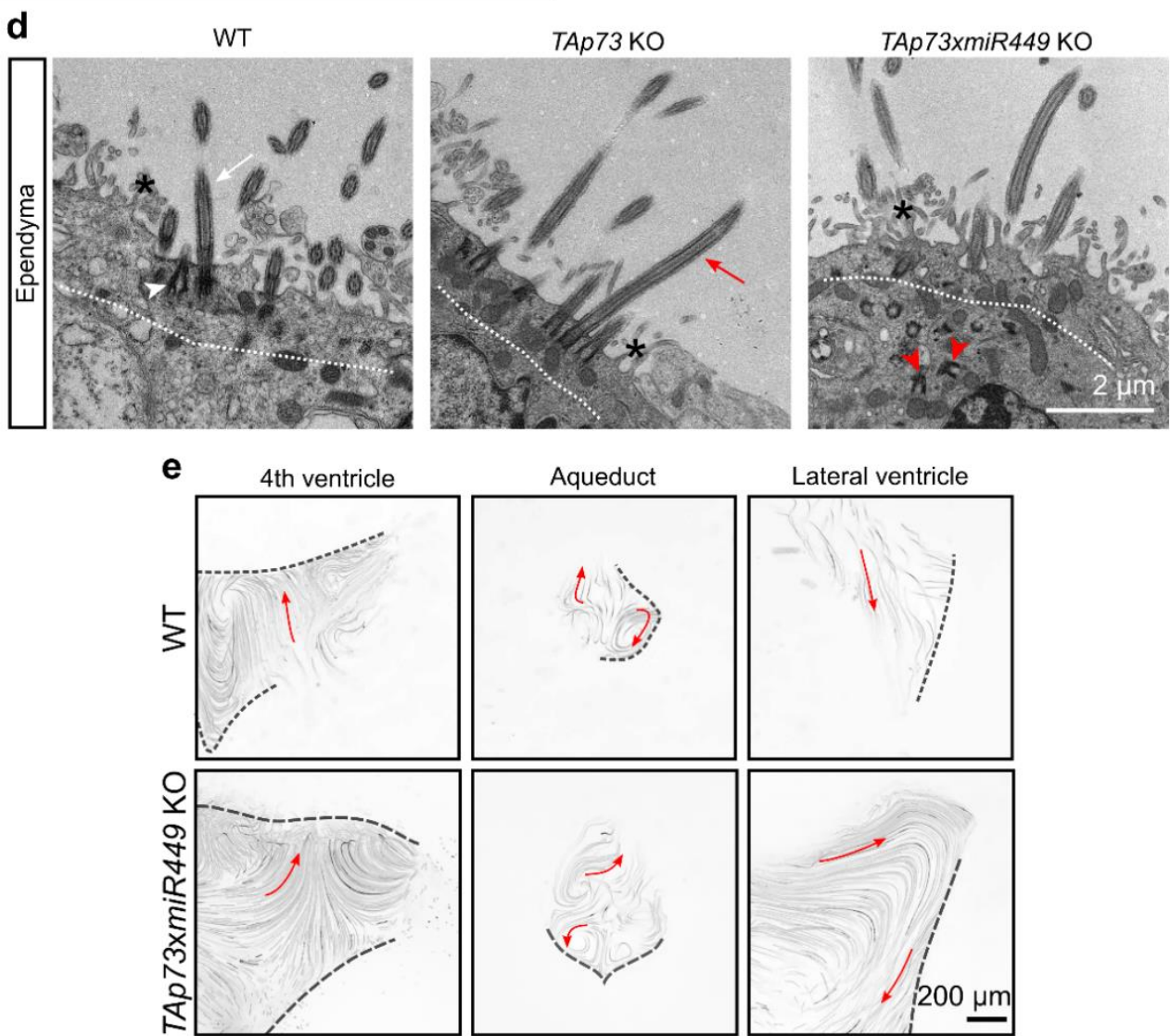

Aqueduct

Lateral ventricle

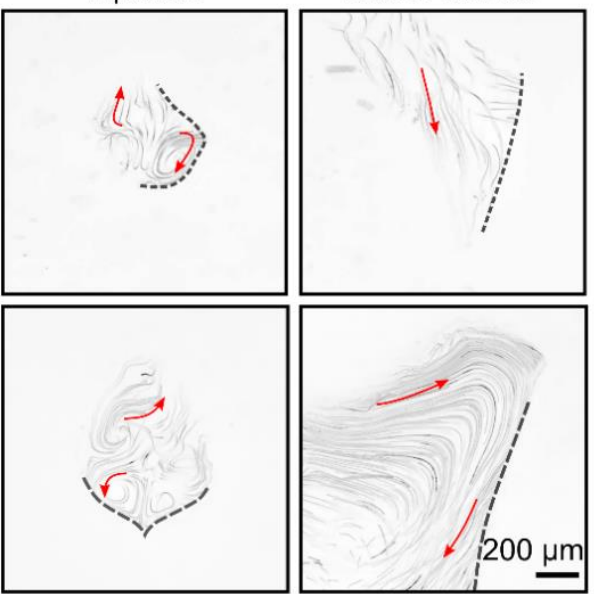

Supplemental Figure 9. 
Supplemental Figure 9: TAp73xmiR449 KO mice possess reduced numbers of CP cilia, but not of ependymal cilia.

a) Representative H\&E images of sagittal brain sections from WT, TAp73 KO, miR449 KO, and TAp73xmiR449 KO mice. Arrows point at the lateral ventricles. Notice the enlarged lateral ventricle in TAp73xmiR449 KO animals. b) Representative images of the expression of ARL13B (arrowheads mark cilia) of the CP of the 4th and lateral ventricles from WT, miR449 KO, and TAp73xmiR449 KO mice. c) Expression of Ac-a-TUB (green) in the lateral ventricle of WT and TAp73xmiR449 KO mice. DAPI staining (blue) labels nuclei. Boxed regions are magnified and illustrate similar cilia number in WT and TAp73xmiR449 KO animals. d) TEM of ependymal cilia from WT, TAp73 KO, and TAp73xmiR449 KO mice. Red arrowheads indicate mislocalization of BBs. e) Analysis of the movement of fluorescent beads along the ventricular system. Images of maximum intensity projections of representative movies of the $4^{\text {th }}$ ventricle, lateral ventricle, and aqueduct are shown $(n=2)$. Red arrows mark the direction of bead flow. Bracket lines delineate ependymal layer lining the ventricles. 
a

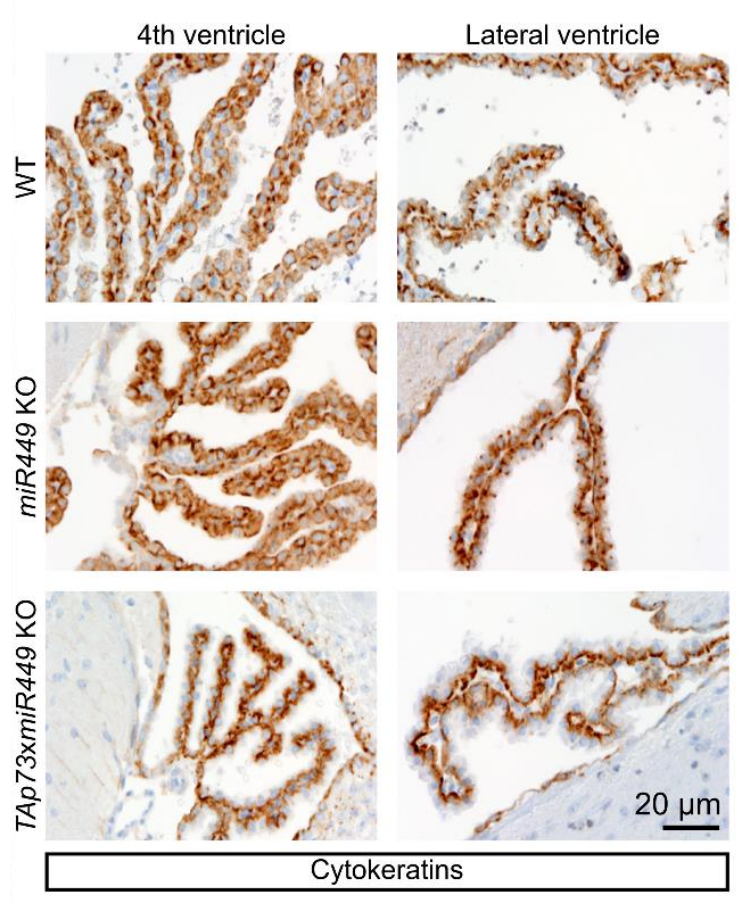

b
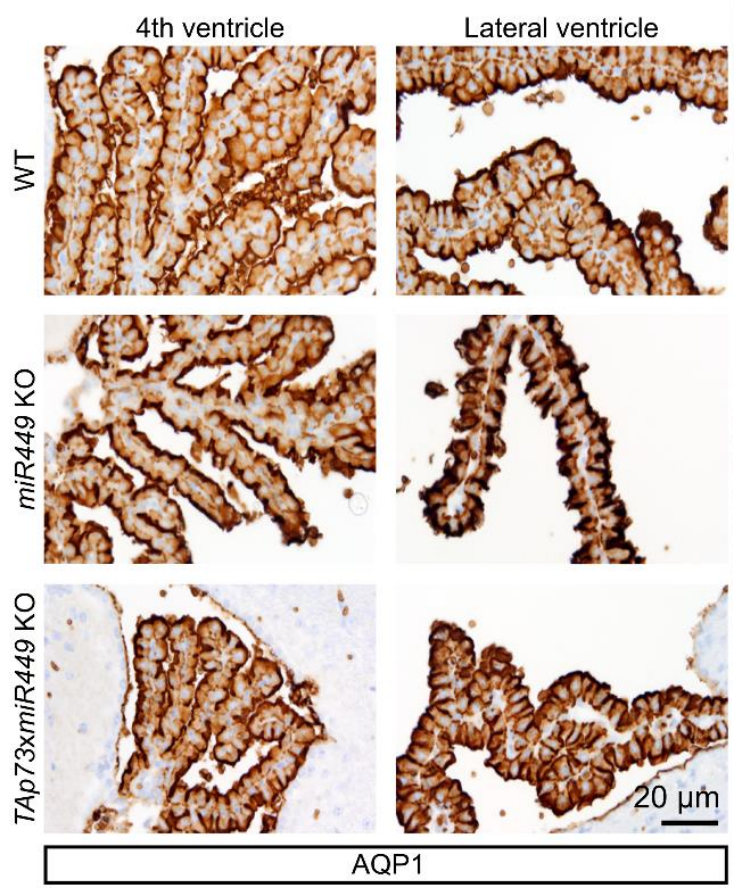

c

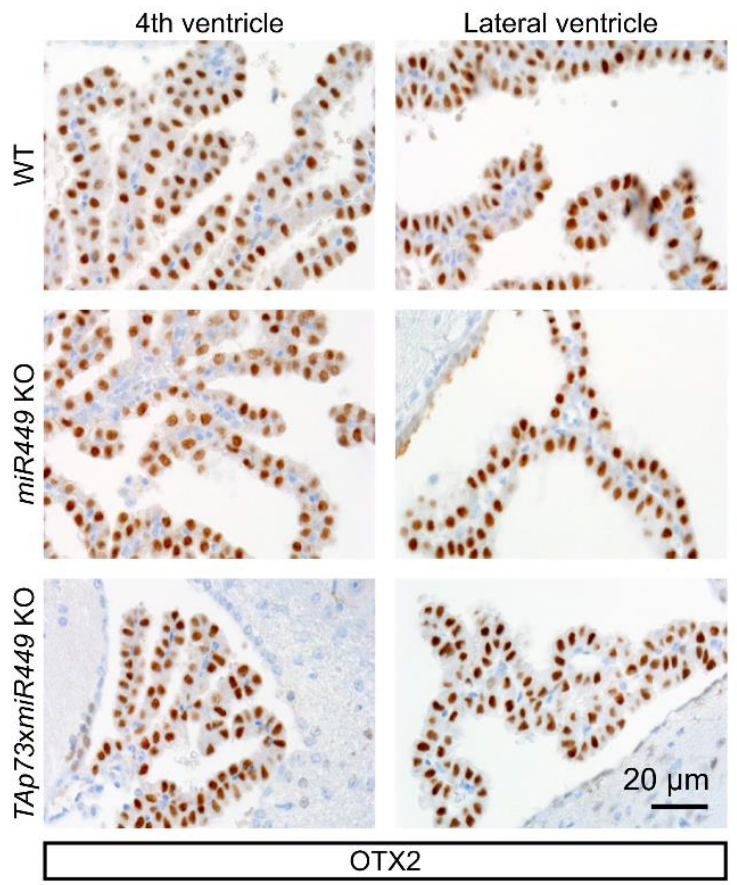

Supplemental Figure 10: Combined loss of TAp73 and miR449 does not affect epithelial differentiation of CP cells.

a - c) Representative images of the expression of Cytokeratins (a), AQP1 (b), and Orthodenticle homeobox 2 (OTX2, c) in CP epithelium of the 4th and lateral ventricles from WT, miR449 KO, and TAp73xmiR449 KO mice. 

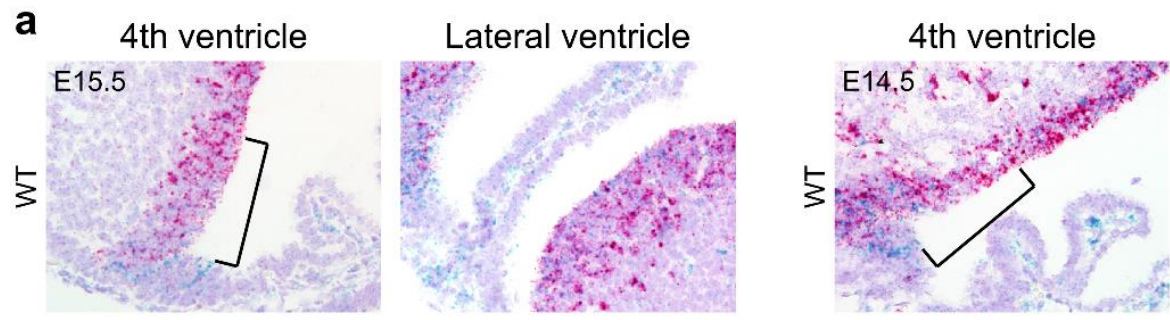

Lateral ventricle
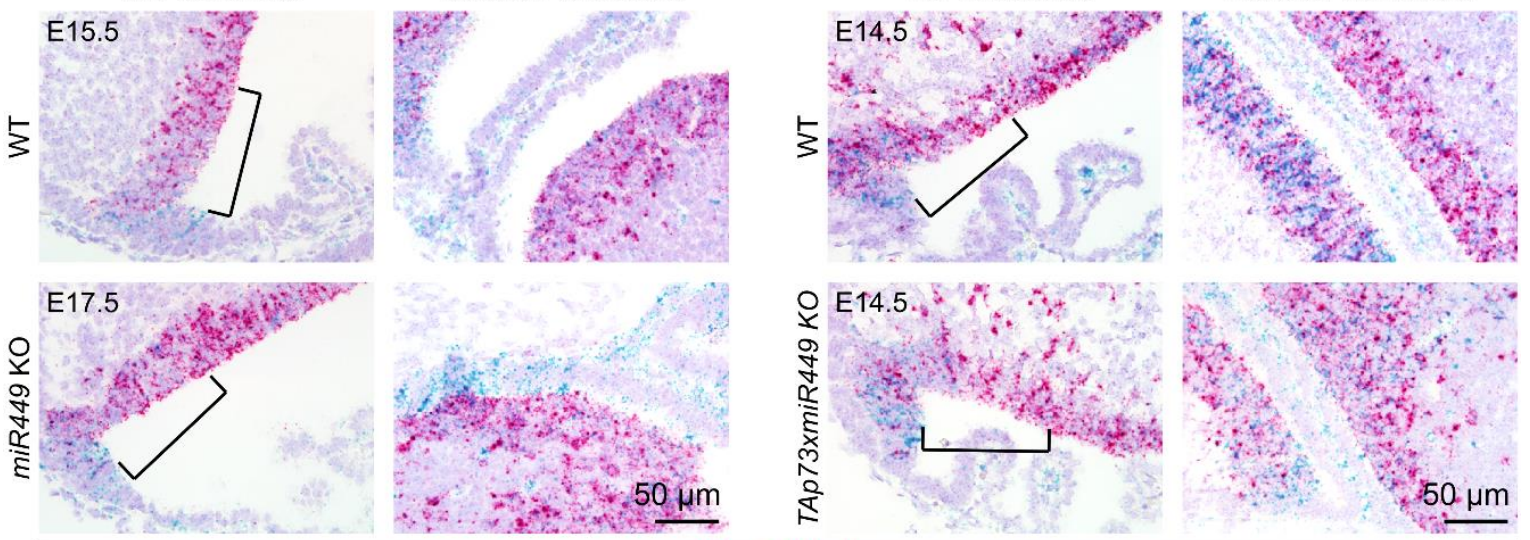

Supplemental Figure 11: Notch signaling is unaltered in developing ventricles of miR449 KO and TAp73xmiR449 KO mice.

a) RNAscope analysis of the expression of Notch targets Hes 1 (blue) and Hes5 (red) in roof plate of 4 th and lateral ventricles of WT, miR449 KO, and TAp73xmiR449 KO mice.

\section{a}

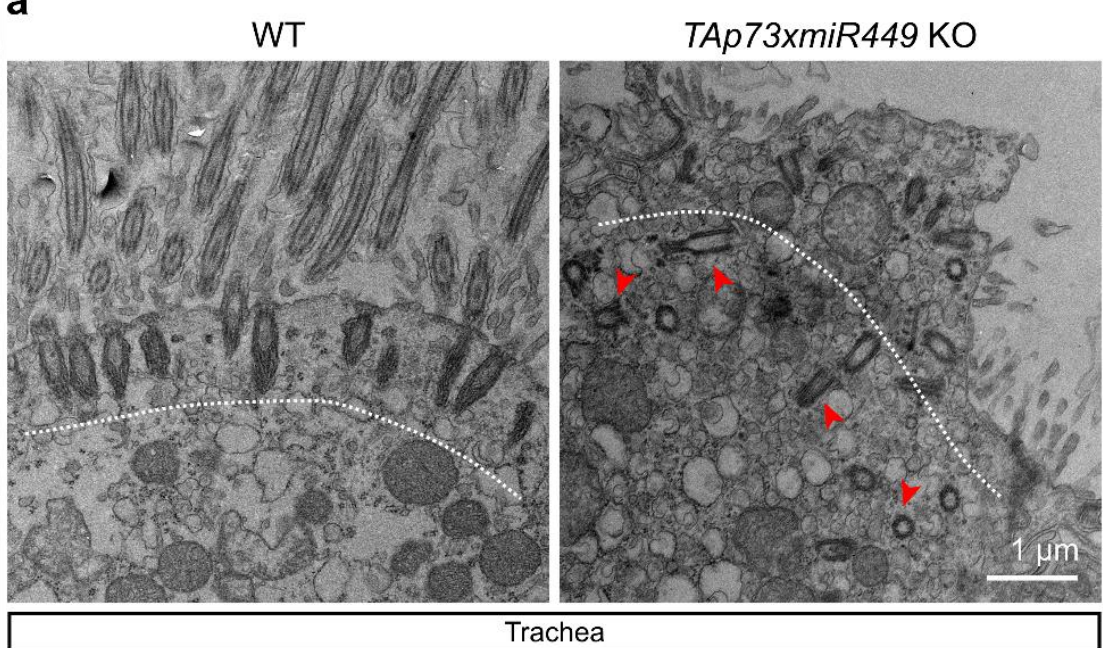

b

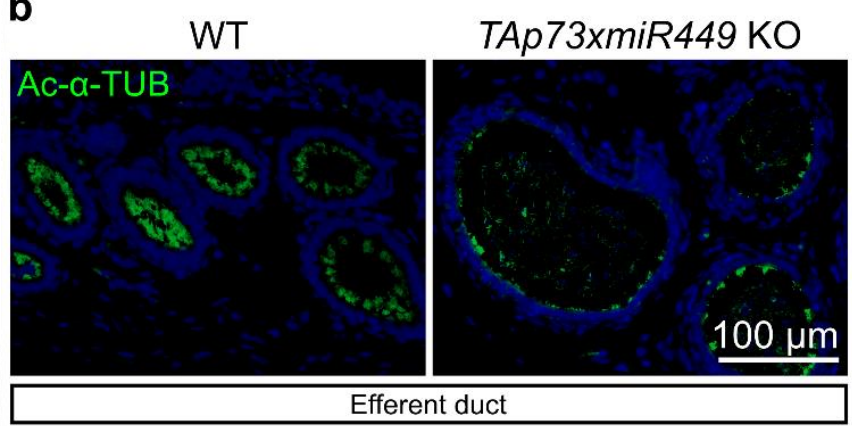

C

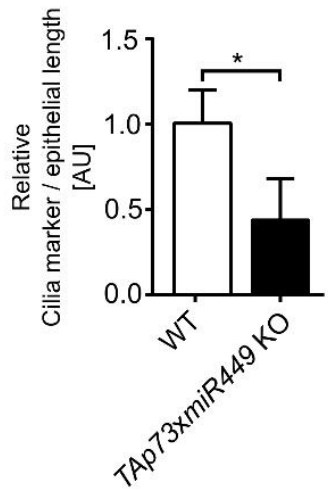

Supplemental Figure 12. 
Supplemental Figure 12: Additional loss of miR449 does not exacerbate ciliary defect in the absence of TAp73 in the airways and efferent ducts.

a) TEM of airway cilia from WT and TAp73xmiR449 KO mice. Red arrowheads indicate mislocalization of BBs. b) Representative images of the expression of Ac-a-TUB (green) are shown in EDs of WT and TAp73xmiR449 KO mice. DAPI staining (blue) labels nuclei. c) Quantitation of Ac- $a-T U B$ signals shown in (a) was normalized to epithelial length ( $n=7$ images from 4 WT mice; $n=6$ images from 3 TAp73xmiR449 KO mice). Data are presented as the mean \pm SEM and relative to the WT group.

a

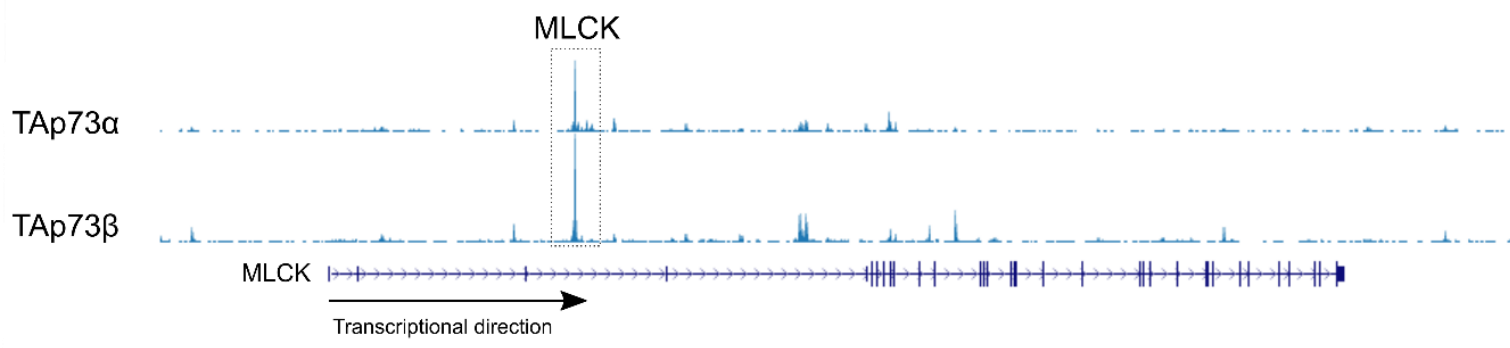

b

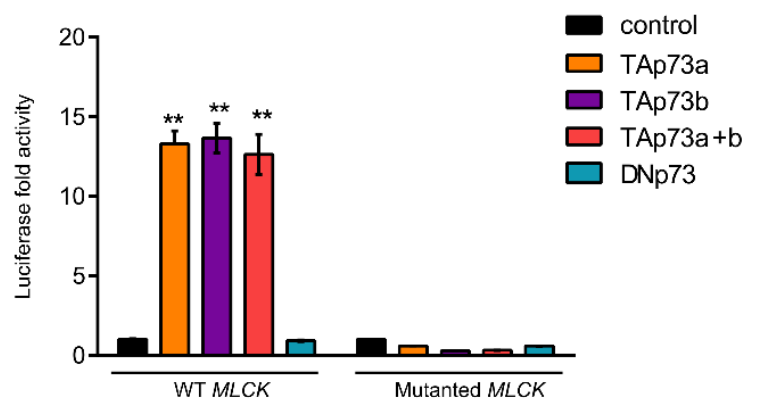

Supplemental Figure 13: Binding of TAp73 is enriched at the genomic locus from MLCK.

a) TAp73 binding at myosin light chain kinase (MLCK) genomic locus is shown in results from ChIP-seq (Koeppel et al., 2011), Geo accession no. GSE15780. Boxed regions mark genomic loci enriched with TAp73 binding. b) Luciferase reporter assay of MLCK regulatory regions containing TAp73 binding motifs. Mutant vector lacks TAp73 binding site (Table 3). WT or mutant luciferase vectors were co-transfected with empty vector (control), or vectors expressing different $\mathrm{p} 73$ isoforms. Fold changes in luciferase activities relative to those of the control vector are shown $(n=3)$. Data are presented as the mean \pm SEM. 


\subsection{Chapter 2: miR449 protects airway cilia and healthy lung aging, thereby preventing COPD}

Merit Wildung ${ }^{1}$, Christian Herr ${ }^{2}$, Dietmar Riedel ${ }^{3}$, Maren Cors ${ }^{1}$, Hataitip Tasena ${ }^{4,5}$, Mihai Alevra $^{6,7}$, Cornelia Wiedwald ${ }^{1}$, Naira Movsisyan ${ }^{8}$, Maike Schuldt ${ }^{1}$, Larisa Volceanov-Hahn ${ }^{1}$, Sharen Provoost ${ }^{9}$, Tabea Menchen ${ }^{10}$, Diana Urrego ${ }^{8}$, Bernard Freytag ${ }^{1}$, Julia Wallmeier ${ }^{10}$, Christoph Beisswenger ${ }^{2}$, Robert Bals ${ }^{2}$, Maarten van den Berge ${ }^{5,11}$, Corry-Anke Brandsma $^{4,5}$, Tania Maes ${ }^{9}$, Stefan Andreas ${ }^{1}$, Irene H. Heijink ${ }^{4,5,11}$, Luis Pardo ${ }^{8}$ and Muriel Lizé $^{1^{*}}$

* $=$ corresponding author

1) Molecular \& Experimental Pneumology Group, Clinic for Cardiology and Pneumology, University Medical Center Goettingen, Germany

2) Department of Internal Medicine V, Saarland University, Homburg, Germany

3) Electron Microscopy, Max-Planck-Institute for Biophysical Chemistry, Goettingen, Germany

4) University of Groningen, University Medical Center Groningen, Department of Pathology and Medical Biology, Groningen, The Netherlands

5) University of Groningen, University Medical Center Groningen, Groningen Research Institute for Asthma and COPD, Groningen, The Netherlands

6) Department of Neurophysiology and Cellular Biophysics, Goettingen University, Goettingen, Germany

7) Institute of Neuro- and Sensory Physiology, Goettingen University, Goettingen, Germany

8) Oncophysiology Group, Max-Planck-Institute of Experimental Medicine, Goettingen, Germany

9) Laboratory for translational Research in Obstructive Pulmonary Diseases, Department of Respiratory Medicine, Ghent University Hospital, Ghent, Belgium

10) Department of Pediatrics, University Hospital Muenster, Muenster, Germany

11) University of Groningen, University Medical Center Groningen Groningen Department of Pulmonology, Groningen, The Netherlands

Manuscript in preparation

\section{Contribution to manuscript}

M. W. conducted experiments and data analysis for the following figures:

Figure 29c; Figure 30a-d; Figure 31a; Figure 32a-d; Figure 33a-c; Figure 34a-c; Figure 35ac; Figure 37c-f; Figure 39a, d-f; Figure 40c, d; Figure 41; Supplemental Figure 14a; Supplemental Figure 15a, b; Supplemental Figure 16b; Supplemental Figure 17a, b; Supplemental Figure 18a.

M. W. arranged the figures and contributed to manuscript writing.

C.H., R.B, and C.B. performed lung function analysis, NTHi and CS mouse models. D.R. identified and quantified abnormal cilia ultrastructure by EM. M.C., L.V-H., and B.F. contributed to the target validation, M.C., M.S., T.Me, and J.W. contributed to analysis of the ciliary phenotype. H.T., I.H., C-A.B, and M.B. contributed to the analysis COPD patient data. M.A. analyzed mucociliary clearance assay. C.W. performed RNA isolation and qPCRs. N.M. and D.U performed ciliary disassembly assay. S.P. and T. Ma. contributed to the analysis of DEP-treated lungs. S.A. contributed to interpretation and supported the group. L.P., I.H., M.L. and contributed to manuscript writing. M.L. designed and initiated the study and acquired the required funding. 


\subsubsection{Cilia-associated genes positively correlate with miR449 in COPD patients}

Using genome-wide mRNA and miRNA expression profiles of bronchial biopsies collected from 57 COPD patients (Figure 28a, Supplemental Table 1), we identified 1,444 genes positively correlated with miR449a (FDR<0.05) (data not shown). To identify biological processes in which miR449a-correlated genes play a role, GSEA (Subramanian et al., 2005) was performed on a list of genome-wide genes ranked by the strength of their correlation with miR449a. Several gene sets related to ciliogenesis were significantly enriched among the miR449a-positively correlated genes, including the top 5 processes depicted in Figure 28b. As expected from earlier observations (Lizé et al., 2010a; Song et al., 2014; Wu et al., 2014; Otto et al., 2017), genes positively correlated with the other members of the miR-34/449 family including miR34b,c but not miR34a also showed an enrichment for cilia-associated processes (Supplemental Table 2 - 4). To identify specific genes through which miR449a regulates ciliogenesis, we compared 1,444 miR449apositively correlated genes with a previously published list of 581 cilia-associated genes (Nemajerova et al., 2016) and found 135 overlapping genes associated with structure, maintenance, and function of cilia (Figure 28c). One of the most significant gene was $D N A / 1$, a component of the outer dynein arm, which is involved in cilia beating (Guichard et al., 2001; Zariwala et al., 2006; Chhin et al., 2009) (Figure 28c). To conclude, miR449a is positively correlated with cilia-associated genes in COPD patients. 
a

COPD patients

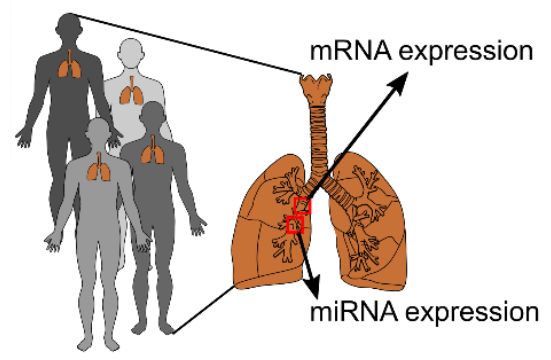

b

Top 10 GO terms among miR449a-positivly correlated genes

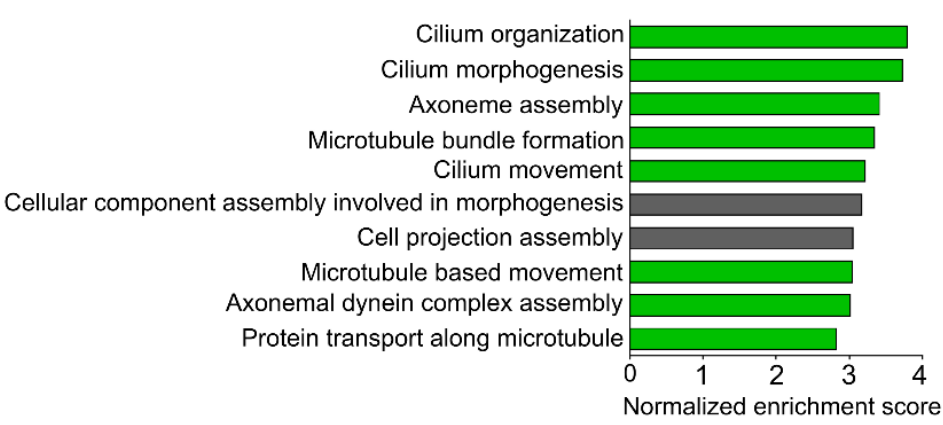

C

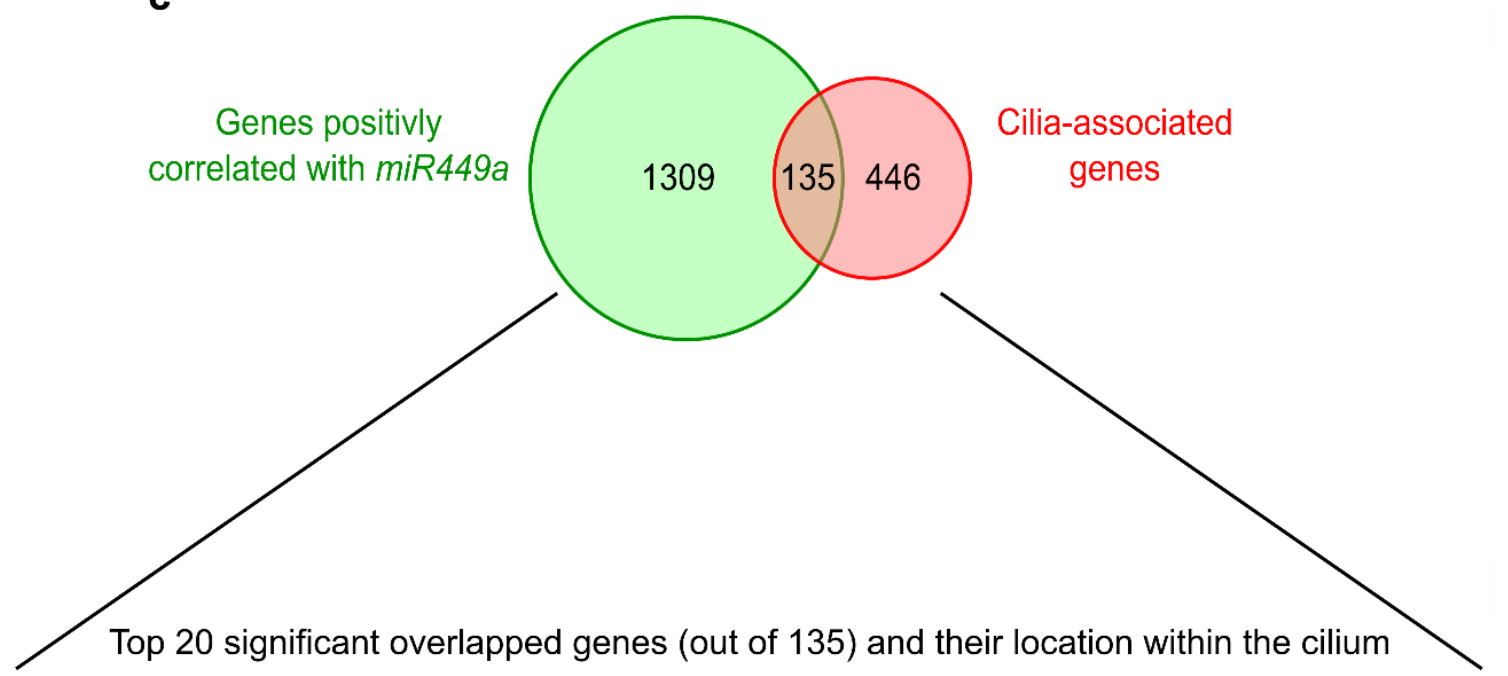

\begin{tabular}{|l|c|c|}
\hline \multicolumn{1}{|c|}{ Gene } & p-value & FDR \\
\hline DYNLT1 & $1.8 \mathrm{E}-08$ & $3.9 \mathrm{E}-05$ \\
\hline STIL & $3.5 \mathrm{E}-08$ & $6.2 \mathrm{E}-05$ \\
\hline STK36 & $1.0 \mathrm{E}-07$ & $1.0 \mathrm{E}-04$ \\
\hline TTLL4 & $3.7 \mathrm{E}-07$ & $1.9 \mathrm{E}-04$ \\
\hline IFT140 & $2.3 \mathrm{E}-06$ & $4.6 \mathrm{E}-04$ \\
\hline CCDC65 & $2.4 \mathrm{E}-06$ & $4.6 \mathrm{E}-04$ \\
\hline GAS8 & $2.6 \mathrm{E}-06$ & $4.9 \mathrm{E}-04$ \\
\hline DNAI1 & $3.1 \mathrm{E}-06$ & $5.5 \mathrm{E}-04$ \\
\hline STX3 & $3.6 \mathrm{E}-06$ & $5.8 \mathrm{E}-04$ \\
\hline ZMYND10 & $4.0 \mathrm{E}-06$ & $5.9 \mathrm{E}-04$ \\
\hline RSPH9 & $4.4 \mathrm{E}-06$ & $6.1 \mathrm{E}-04$ \\
\hline RSPH3 & $4.8 \mathrm{E}-06$ & $6.4 \mathrm{E}-04$ \\
\hline KIF3B & $6.0 \mathrm{E}-06$ & $7.2 \mathrm{E}-04$ \\
\hline ROPN1L & $9.6 \mathrm{E}-06$ & $9.2 \mathrm{E}-04$ \\
\hline AKAP14 & $9.8 \mathrm{E}-06$ & $9.3 \mathrm{E}-04$ \\
\hline DNAI2 & $9.9 \mathrm{E}-06$ & $9.4 \mathrm{E}-04$ \\
\hline RSPH1 & $1.1 \mathrm{E}-05$ & $1.0 \mathrm{E}-03$ \\
\hline DNAL11 & $1.1 \mathrm{E}-05$ & $1.0 \mathrm{E}-03$ \\
\hline TTLL5 & $1.3 \mathrm{E}-05$ & $1.1 \mathrm{E}-03$ \\
\hline CBY1 & $2.0 \mathrm{E}-05$ & $1.3 \mathrm{E}-03$ \\
\hline
\end{tabular}

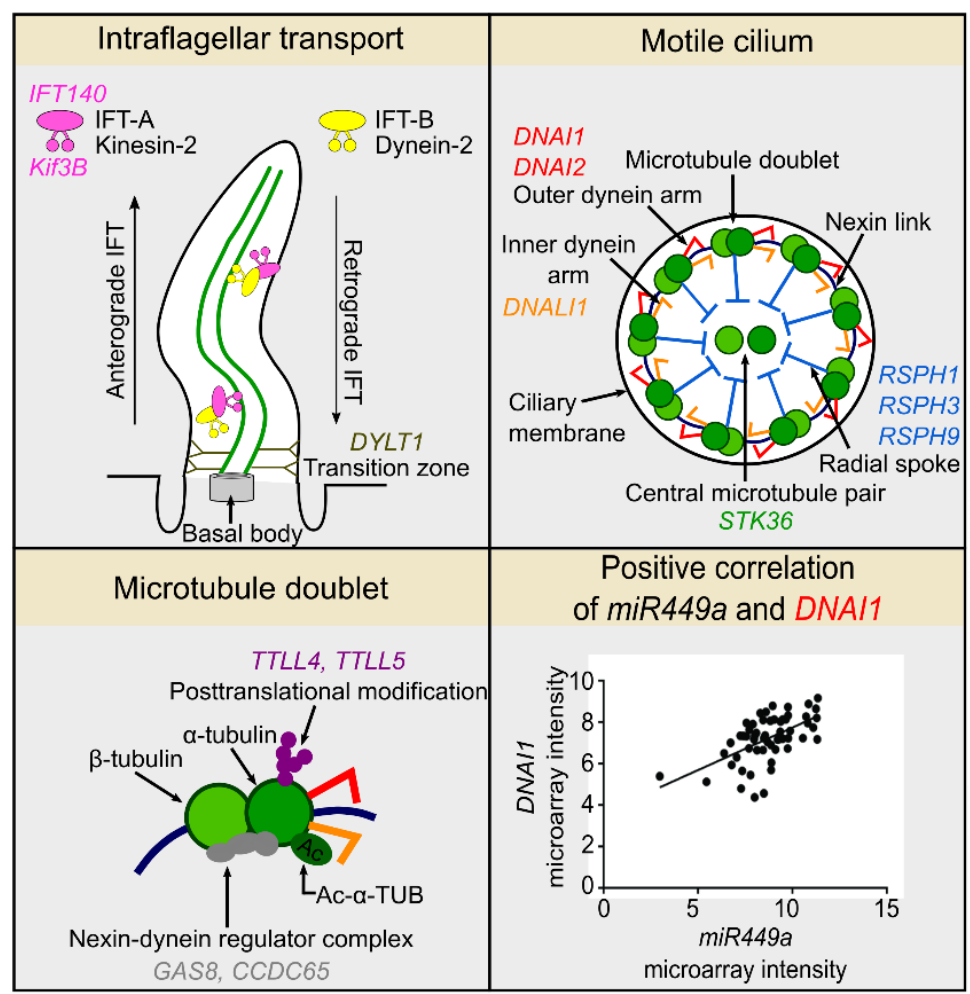

Figure 28. 
Figure 28: miR449 expression is positively correlated with the ciliation status in COPD patients. a) Illustration of the GLUCOLD study design. Bronchial biopsies from 57 COPD patients "with moderate to severe symptoms" were taken and microarray analysis was performed for mRNA and miRNA profiling. b) Top 10 significant biological processes enriched among miR449a-positively correlated genes in COPD patients. GSEA was performed using the list of genes ranked based on the strength of their correlation with miR449a and the Gene Ontology dataset for biological processes. All gene sets are significant with FDR $<0.001$. Green bars highlight cilia-associated processes. c) 135 genes, which are positively correlated with miR449a expression in COPD patients, are cilia-related genes (Nemajerova et al., 2016). Left panel: Table shows the top 20 significant overlapped genes (out of 135). Right panel: Schematic illustrations of the IFT, cross section of a motile cilium, and the microtubule doublet structure highlight the location of some of the genes positively correlated with miR449a. Lower right panel: Positive correlation of miR449a and DNAl1. Significance was defined by FDR $<0.05$.

\subsection{2 miR449 is upregulated during airway epithelial differentiation and upon bronchial challenges}

The positive correlation between miR449 and ciliary genes in COPD patients matches well with the expression of miR449 in multiciliated epithelia (Lizé et al., 2010a; Marcet et al., 2011; Song et al., 2014). To confirm miR449 expression during the formation of multiciliated epithelia, we assessed its expression by ISH on mouse embryos (E18.5) and found an enrichment of miR449a in multiciliated organs, notably in the developing bronchial epithelium (Figure 29a). Previous studies already demonstrated that miR449 cluster members are far more strongly upregulated than the miR34 loci during mucociliary differentiation (Lizé et al., 2010a; Marcet et al., 2011). We confirmed this in our present study using human airway epithelial cells from healthy donors, which were cultured at ALI for 23 days (Figure 29b). This is further supported by the result of RNA-seq data from ALI cultures of MTECs (Nemajerova et al., 2016) (Figure 29c). The onset of miR449 expression was accompanied by the expression of other well-known regulators of multiciliogenesis such as Gemc1, Mcidas, and Foxj1 (Spassky and Meunier, 2017), whereby miR449 total read counts were most strongly induced compared to other pro-ciliogenic regulators including miR34b,c and peaked between d4 and d7 (Figure 29c).

Altogether, this suggests a much stronger contribution of miR449 to mucociliary differentiation than its homologues, and we thus, focused our subsequent analyses on elucidating the role of miR449 in the regeneration of the multiciliated airway epithelium. 
a

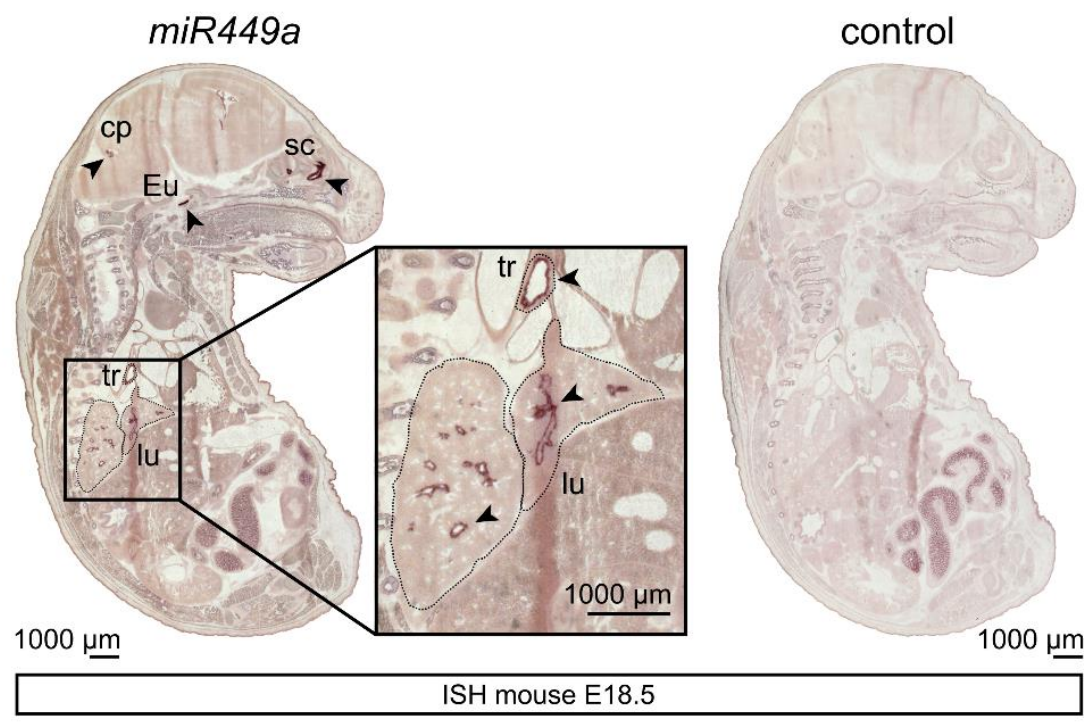

b

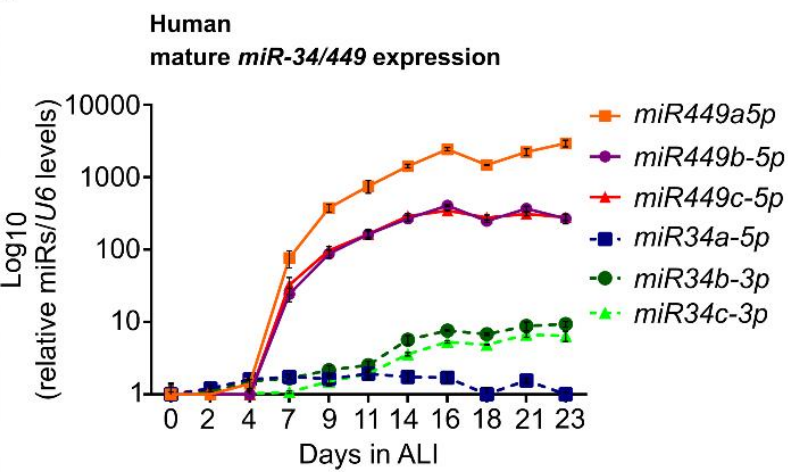

C

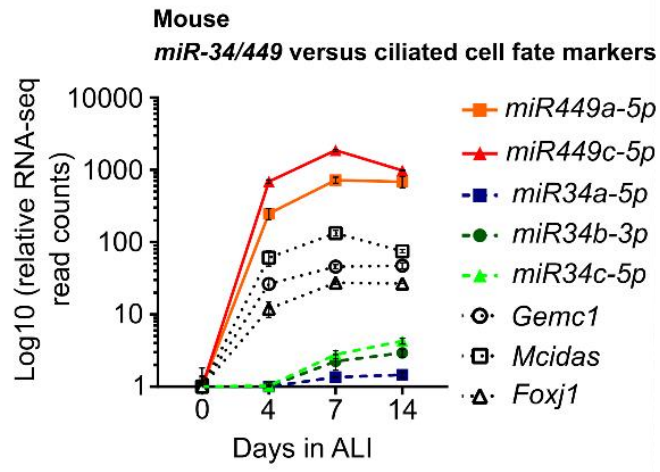

Figure 29: miR449 is induced upon mucociliary differentiation in vitro.

a) ISH of sections from mouse embryos (E18.5) with a miR449a probe (left) and a secondary antibody ctrl (right). Arrowheads indicate positive staining for miR449a in multiciliated organs. Magnification highlights the

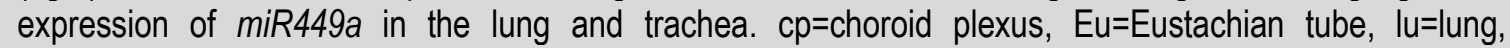
$\mathrm{sc}=$ supranasal cavity, and tr=trachea. b) Mature miR-34/449 levels in human airway epithelial cells undergoing multiciliated differentiation at ALI. do serves as reference. $n=3$ per time point. Data points for miR34a and miR449a were previously published (Lizé et al., 2010a). c) Total read counts from RNA-seq data (Nemajerova et al., 2016) for miR-34/449 and ciliated cell fate markers (Gemc1, Mcidas, and Foxj1) during differentiation of MTECs at ALI. d0 serves as reference. mRNA species: $n=3$ ALIs per time point; miRNA species: $n=2$ per time point. Data are presented as the mean \pm SEM.

One widely used airway regeneration model consists of the exposure of rodents to naphthalene, which causes extensive club cells exfoliation (van Winkle et al., 1995) and ciliated cell squamation accompanied by motile cilia disassembly (Lawson et al., 2002). Therefore, we treated WT mice with naphthalene and analyzed the lungs at different phases during the regeneration process, which is usually completed after two weeks (Figure 30a). miR449a levels immediately increased on d1 after naphthalene treatment and accumulated further until d7, where miR449a reached its highest expression (Figure 30b).

Pollutants such as DEPs, which are the main component of traffic-related particulate matter, also represent environmental challenges for the airway epithelium. DEPs are known to 
cause acute airway inflammation, reduced cilia beat frequency (Grove et al., 2018), as well as changes in the epithelial composition by reducing ciliated and non-ciliated cells (Harrod et al., 2005). The link between DEPs and ciliated epithelial regeneration prompted us to analyze miR449 expression after exposure to DEPs in WT mice (Figure 30c). Indeed, transcript levels of the miR449 host gene $C d c 20 b$ and miR449a itself were strongly upregulated in DEP-treated WT mice (Figure 30d).

Overall, the data show an upregulation of miR449 during differentiation and upon regenerative challenges of multiciliated airway epithelia, indicating a potential role in the regulation of these processes.

a

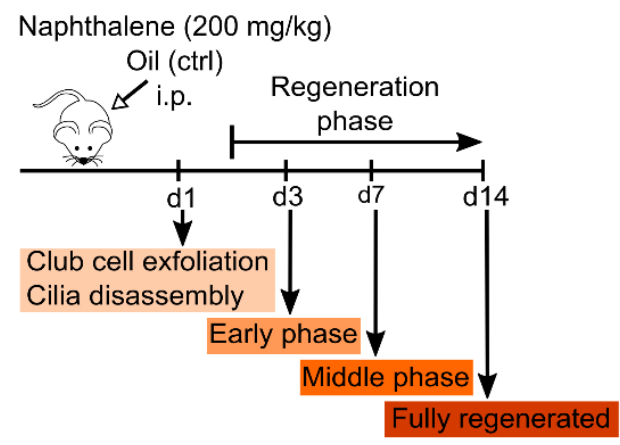

C

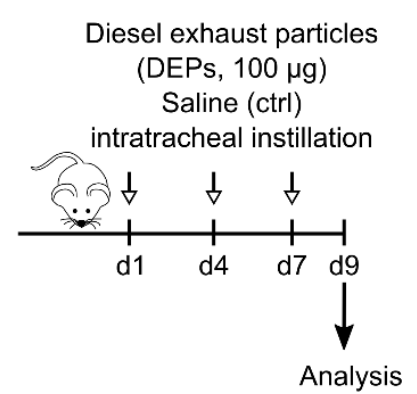

b

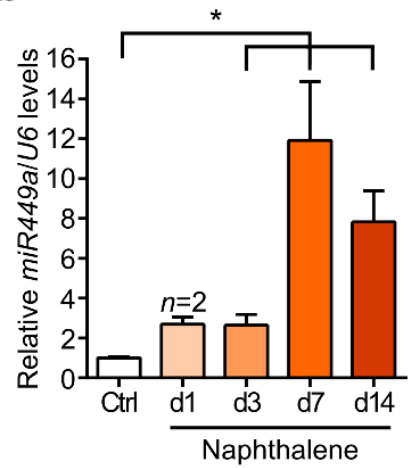

d

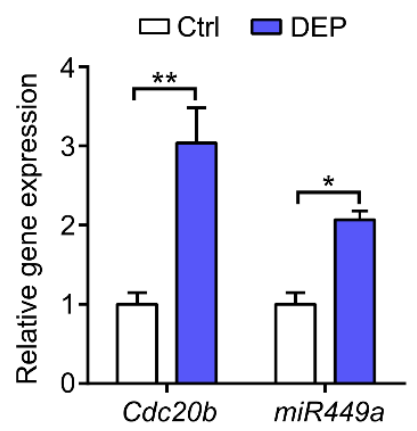

Figure 30: miR449 is elevated in the lungs upon regenerative challenges.

a) Schematic overview of naphthalene-induced airway injury and the regeneration timeline. Mice were injected (i.p.) once with naphthalene $(200 \mathrm{mg} / \mathrm{kg}$ ) or oil (ctrl). b) Mature miR449a levels were quantified in WT lungs at different time points after naphthalene treatment. $n=3$ (ctrl), $n=2$ (d1), and $n=5$ (d3, d7, d14) mice per group. Ctrl levels serve as reference. c) Overview of DEP-induced acute lung inflammation. Mice were instilled intratracheally with DEPs $(100 \mu \mathrm{g})$ or saline (ctrl) on d1, d4, and d7, and analyzed at d9. d) $\mathrm{Cdc} 20 \mathrm{~b}$ as well as mature miR449a levels were quantified in ctrl and DEP-treated lungs, and normalized to $36 b 4$ or U6, respectively. Ctrl levels serve as reference. $n=6$ (ctrl), $n=8$ (DEP) mice per group. Data are presented as the mean \pm SEM. 


\subsection{3 miR449 deficiency reduces ciliation in Air-liquid interface cultures}

To further elucidate the role of miR449 in mucociliary differentiation and lung homeostasis, we used the miR449 KO (miR449- from here one) mouse model, where only miR449a, $b$ and $c$ (miR449 from here on) are depleted, but not miR34a nor miR34b,c (Song et al., 2014) (Figure 31a and b). It was shown that miR34b,c are upregulated upon miR449 depletion and can compensate for loss of miR449 in testis (Bao et al., 2012a; Wu et al., 2014). Consistently, miR449-/ mice are fertile, while miR34b, $c^{-/} \times$miR449-/ mice are infertile (Song et al., 2014; Wu et al., 2014; Otto et al., 2017). In contrast to testes, lungs of miR449-/ mice did not show elevated levels of miR34 members (Figure 31b), as previously reported (Wu et al., 2014). Thus, it is possible to study miR449-mediated ciliated regeneration and respiratory function without effects attributable to miR34 changes in these mice.

a

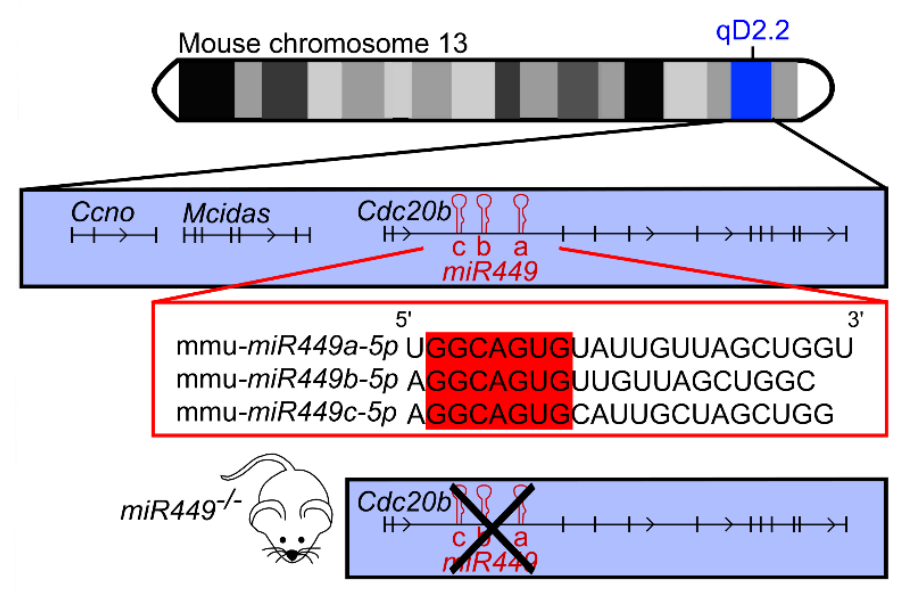

b

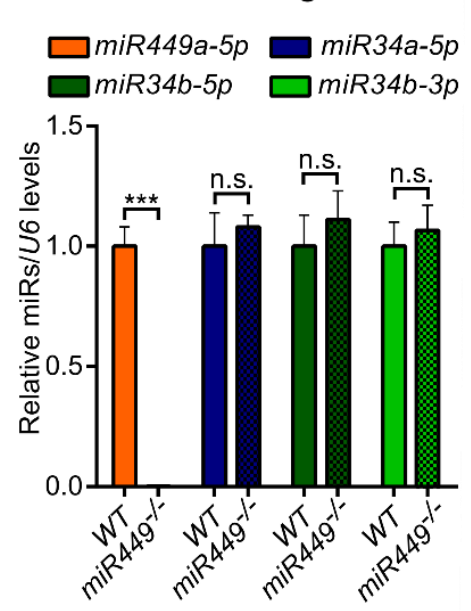

Figure 31: $m i R 34$ expression is not induced in lungs from $m i R 449^{-1-}$ mice.

a) Schematic representation of murine chromosome 13, harboring the genomic region of Ccno, Mcidas, $C d c 20 b$, and miR449 (plus strand). The miR449 cluster is located within the second intron of $C d c 20 b$. Seed sequences are highlighted in red. miR449a, $b$, and $c$ are depleted in miR449--mice without impairing Cdc20b. b) Relative mature miR-34/449 species expression in lungs from WT and miR449--mice. Taqman qPCR, $n$ $=5$ mice per genotype. Data are presented as the mean \pm SEM.

We analyzed cilia quantity and length in ALI cultures derived from WT and miR449/- mice at d6, where miR449 levels reached its highest expression in WT cells (Figure 29c). IF staining for the axonemal cilia markers Ac- $\alpha-$ TUB and DNAl1 revealed a strong reduction in the number and length of motile cilia in miR449-/ compared to WT cultures (Figure 32a $\mathrm{d}$, Supplemental Figure 14a). Thus, miR449 deficiency impairs the generation of a multiciliated epithelium in vitro. 

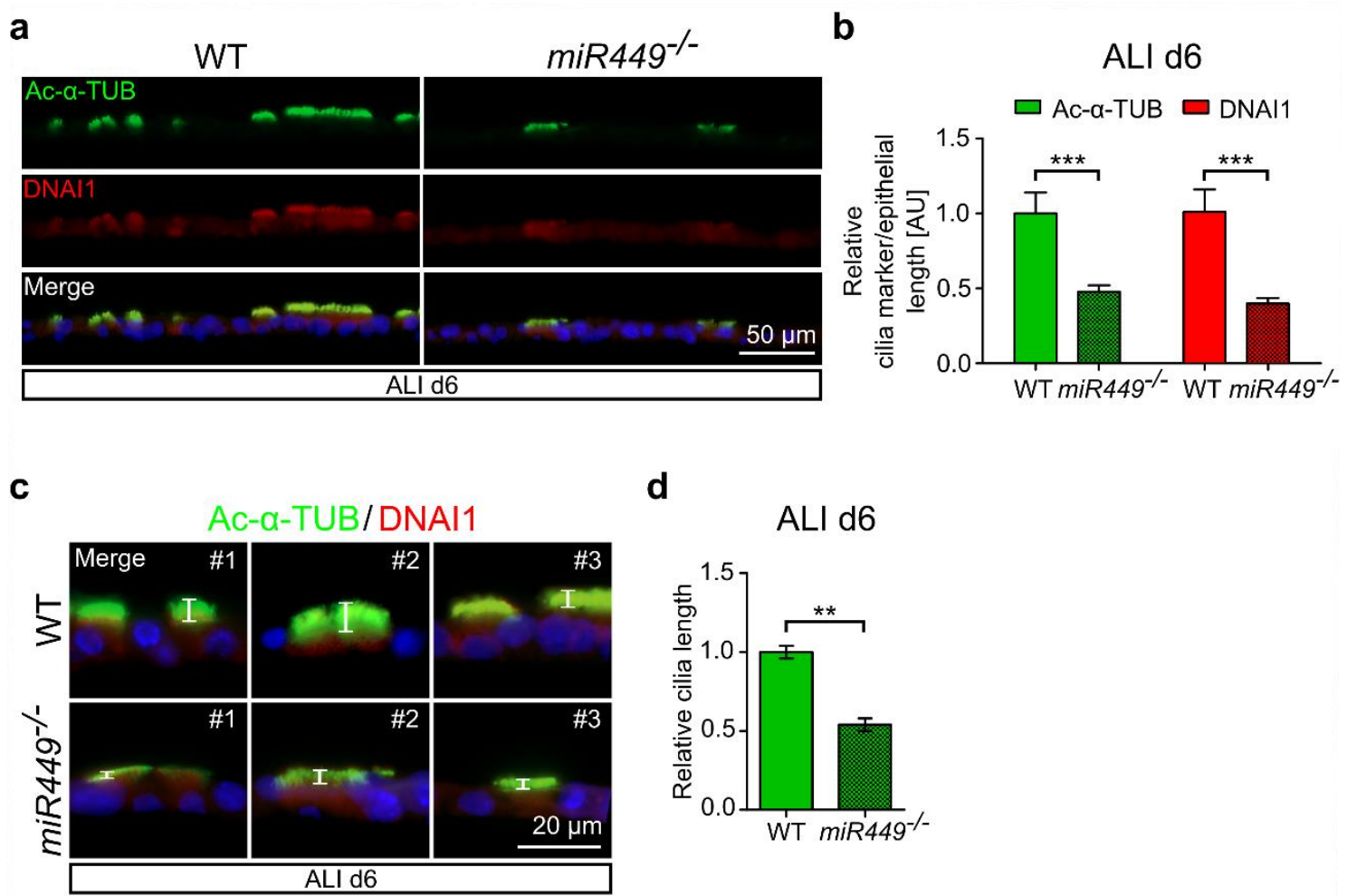

d

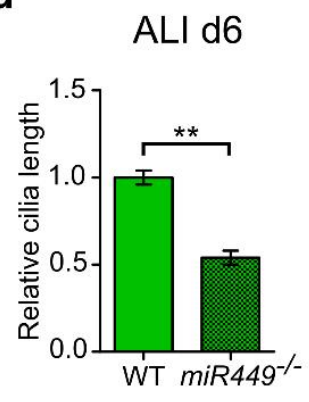

Figure 32: miR449 depletion reduces ciliation in airway cell cultures.

a) WT and miR449- ALI cultures (d6) were stained for axonemal cilia markers Ac-a-TUB (green) and DNAl1 (red). Nuclei were counterstained with DAPI (blue). For more images see Supplemental Figure 14a. b) Quantification of IF signal of cilia markers per epithelial length of ALIs shown in (a) relative to WT. $n=63$ (WT) and $n=99$ (miR449--) images from 3 WT and 3 miR449-- cultures ( 3 mice per culture), respectively. c) Representative magnifications from (a) highlighting cilia length (white bars). d) Relative cilia length measured from (c). Data are presented as the mean \pm SEM.

\subsection{4 miR449 is required for bronchial epithelial regeneration}

Generation and balanced regeneration of the multiciliated airway epithelium is of utmost importance to respiratory function since the airways are constantly exposed to environmental insults. As mucociliary differentiation is impaired in ALI-differentiated epithelial cells from miR449- mice and miR449 is upregulated upon environmental challenges, we assessed the ciliated bronchial epithelial regeneration potential of miR449'- mice after naphthalene treatment (Figure 30a). Airway ciliation was assessed by IF and immunoblot staining for Ac- $\alpha$-TUB and DNAl1, respectively, at different regeneration phases. Both axonemal cilia markers were strongly decreased after injury by naphthalene (d1-d7) and even in the fully regenerated bronchial epithelium (d14) ciliation remained reduced in miR449 $^{-/}$mice (Figure $33 \mathrm{a}$ - c). Microscopy did not reveal any obvious differences in the histology between WT and miR449/- lungs after naphthalene treatment (Supplemental Figure 15a), but miR449-/ mice did not recover to the same extent as WT mice as indicated by their weight loss (Supplemental Figure 15b). These findings indicate that miR449 is required for efficient bronchial epithelial regeneration. 
a

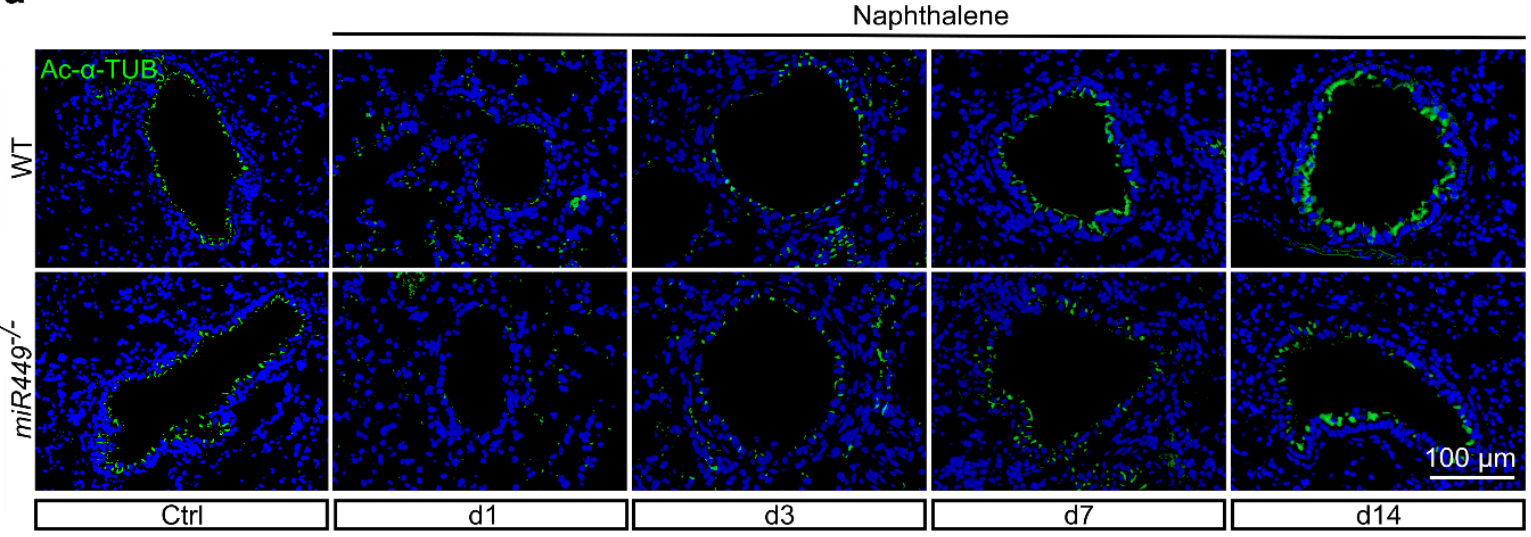

b

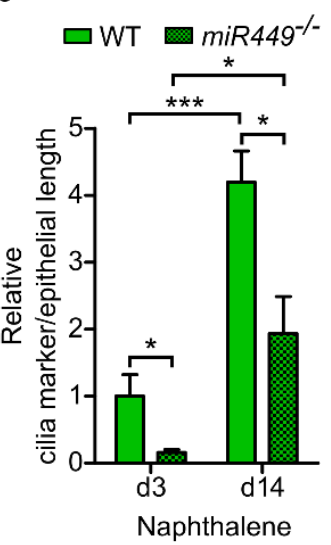

C
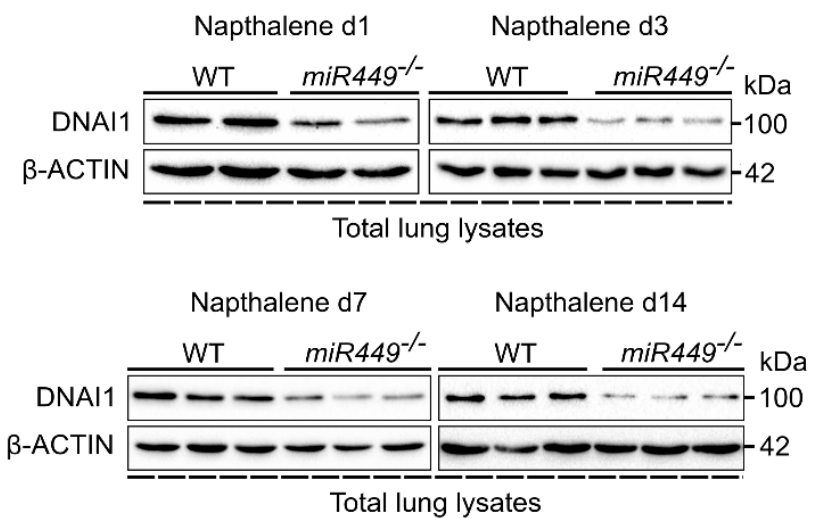

Figure 33: Multiciliated airway regeneration is impaired in miR449-\% mice.

a) Lung sections of ctrl and naphthalene-treated WT and miR449-- mice were stained for the cilium marker Ac-a-TUB (green) and counterstained with DAPI (blue). Cf. Figure 30a for experimental setting. b) Quantification of IF signal of Ac-a-TUB per epithelial length in WT and miR449- lungs at d3 and d14 after naphthalene. WT d3 levels serve as reference. d3: $n=6$ mice/genotype; d14: WT $n=5$ and miR449- $n=3$. Data are presented as the mean \pm SEM. c) The motile cilium marker DNAI1 was quantified in lung lysates from naphthalene-treated WT and miR449-- mice by immunoblot analysis with $\beta$-ACTIN as loading control.

In addition to toxicants, the airway epithelium is continuously exposed to pathogenic bacteria. We next studied whether miR449-- mice are more susceptible to NTHi (Figure 34a), an opportunistic respiratory pathogen affecting cilia beating and often associated with airway diseases such as COPD (King and Sharma, 2015). Motile cilia were indeed reduced in lungs from NTHi-exposed miR449-/ mice compared to NTHi-exposed WT mice, as shown by immunostaining of Ac- $\alpha-T U B$, immunoblot analysis of DNAI1, and transcript levels of the outer dynein arm component Dnah5 (Figure 34b - d). 
a

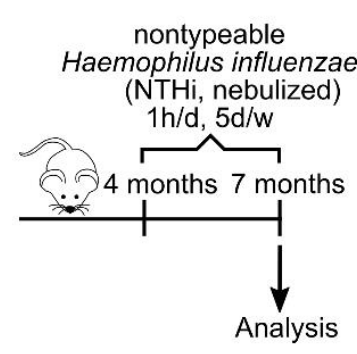

C

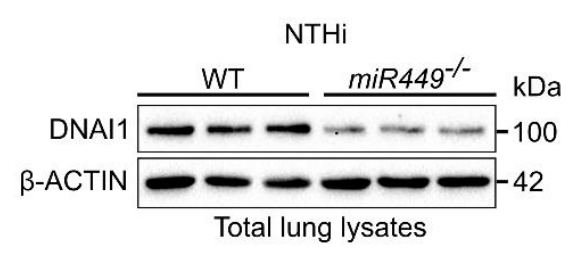

b

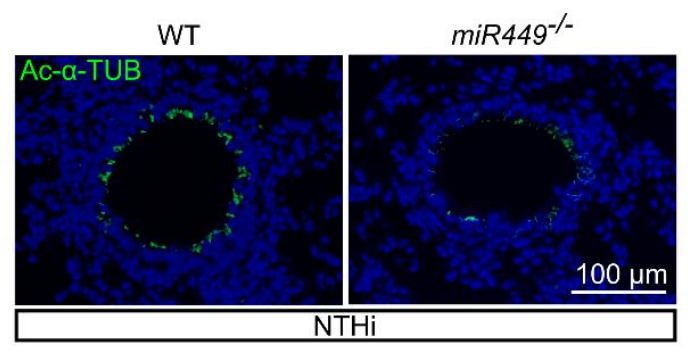

d $\quad \mathrm{NTHi}$

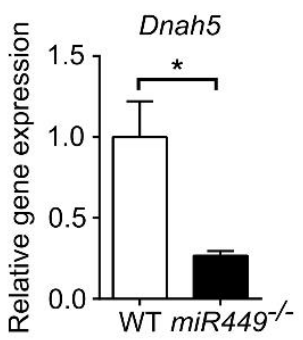

Figure 34: Exposure to NTHi results in a loss of airway cilia in miR449-- mice.

a) Schematic overview of NTHi-induced chronic lung inflammation. WT and miR449-- mice were nebulized with NTHi for $1 \mathrm{~h} / \mathrm{d}$ and $5 \mathrm{~d} /$ week (w) over a period of 3 months. b) Lung sections from NTHi-treated WT and miR449-- mice were stained for Ac-a-TUB (green) and counterstained with DAPI (blue). c) Motile cilium marker DNAl1 was quantified in lung lysates from NTHi-treated WT and miR449- mice by immunoblot analysis with $\beta$-ACTIN as loading control. d) Transcript levels of Dnah5 were quantified by qPCR and normalized to $36 \mathrm{~b} 4$ in NTHi-treated WT and miR449-- lungs. $n=4 /$ genotype. Data are presented as the mean \pm SEM.

As airway challenges never stop as long as we breathe, epithelial regeneration is constantly required during aging. We therefore hypothesized that miR449 depletion might impair airway cilia in aged mice. We stained tracheal sections from 6 months old WT and miR449${ }^{\prime}$ mice for axonemal Ac- $\alpha$-TUB and DNAl1. The comparison showed reduced Ac- $\alpha$-TUB and a nearly complete loss of DNAl1 in aged miR449 ${ }^{-/}$mice (Figure $35 \mathrm{a}$ and b), despite being barrier-maintained under specific pathogen-free conditions including autoclaved cage content and air filtration. Further analysis of protein levels of DNAl1 in total lung lysates from WT and miR449- mice confirmed the strong reduction in ciliation with age upon miR449 depletion (Figure 35c). By contrast, ciliation was not altered in 10 weeks old miR449/- mice (Supplemental Figure 16a and b).

In summary, our data indicate a supportive effect of miR449 on bronchial epithelial regeneration upon various challenges including aging. 
a

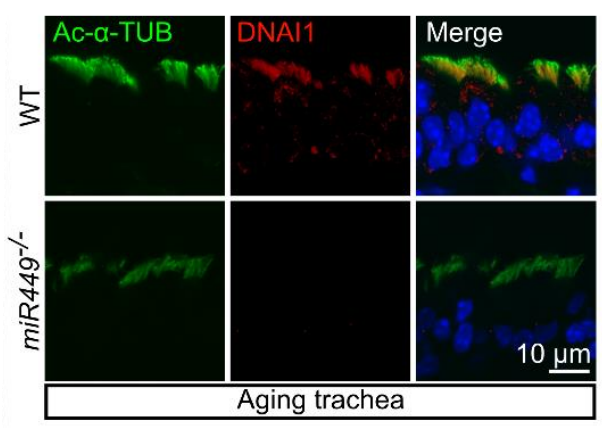

b

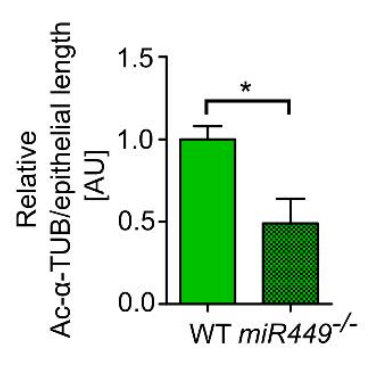

C

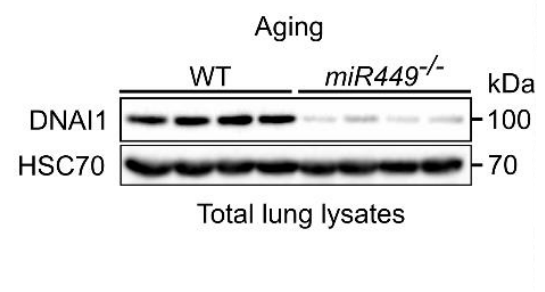

Figure 35: Motile airway cilia are strongly reduced in aged miR449-- mice.

a) Tracheal sections from 6 months old WT and miR449-- mice were stained for the cilia markers Ac-a-TUB (green) and DNAI1 (red). Nuclei were counterstained with DAPI (blue). b) Quantification of the IF signal of Ac- $\alpha$-TUB per epithelial length of tracheal sections shown in (a). WT serves as reference. $n=3$. c) Total lung lysates from 6 months old WT and miR449-- were analyzed by immunoblot using DNAl1 as a motile cilia marker and the constitutive protein $\mathrm{HSC70}$ as a loading control. Data are presented as the mean \pm SEM.

\subsection{5 miR449// mice develop spontaneous COPD accompanied by an increased inflammatory response upon challenge}

Pulmonary tissue and airway regeneration are impaired in COPD, resulting in reduced lung function in the long-term (Kotton and Morrisey, 2014; Staudt et al., 2014), and miR449 has an important positive effect at least on airway epithelial regeneration. Hence, we tested next whether loss of miR449 affects lung function. Spirometry was performed on aged WT and miR449 $\%$ mice inhaling ambient air (AA). As a positive control to induce emphysematous COPD manifestations, WT mice were exposed to CS over a period of 6 months (Shu et al., 2017) (Figure 36a). miR449-/ mice exposed to AA displayed impaired pulmonary function to a similar extent as CS-exposed WT mice, as indicated by a reduced tissue elastance and an increased total lung capacity (Figure $36 \mathrm{~b}$ and $\mathrm{c}$ ). This phenotype was not observed in young miR449 ${ }^{-/}$mice (Supplemental Figure $17 \mathrm{a}$ and b) and did not further increase upon CS exposure of $m i R 449^{-/}$mice (data not shown).

$\mathbf{a}$

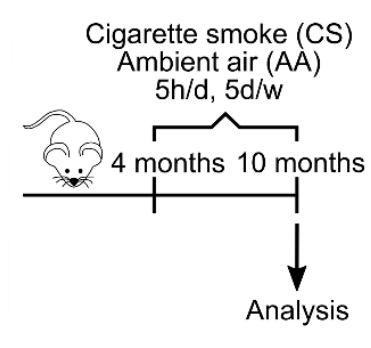

b

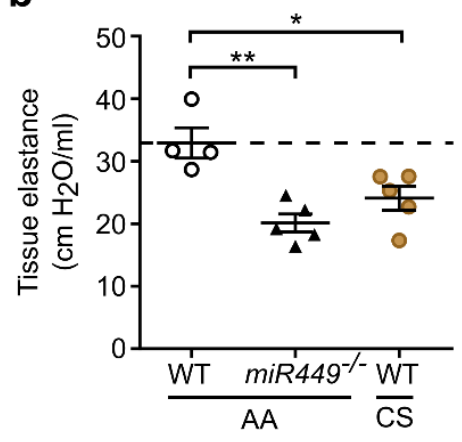

C

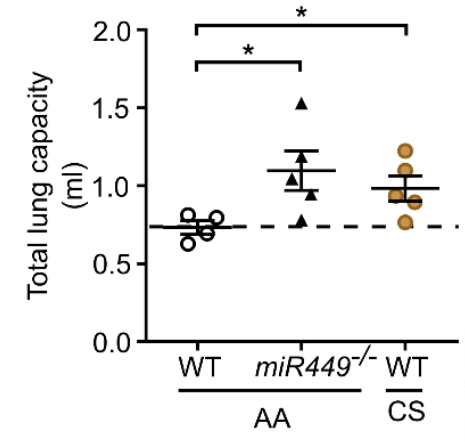

Figure 36. 
Figure 36: miR449 deficiency impairs pulmonary function to a similar extent as overserved in CSexposed WT mice.

a) Schematic overview of the CS-induced COPD model. 4 months old WT miR449-- mice were left at AA or exposed to CS for $5 \mathrm{~h} / \mathrm{d}$ and $5 \mathrm{~d} / \mathrm{w}$ over 6 months. CS-exposed WT mice were used as a positive ctrl for the COPD phenotype. b and c) Pulmonary function was assessed in 10 months old WT, miR449-/, and CSexposed WT mice by measuring tissue elastance (b) and total lung capacity (c) using the FlexiVent system. $n=4-5$ mice/genotype. Data are presented as the mean \pm SEM.

We assessed the mean chord length of serial histological lung sections by stereology and confirmed that aged miR449 ${ }^{--}$mice develop spontaneous emphysematous changes characterized by airspace enlargements (Figure 37a), one of the hallmarks of COPD (Barnes et al., 2015). This is usually accompanied by increased infiltration of macrophages and neutrophils, potentially contributing to an imbalance between proteases and antiproteases in the lungs (Houghton, 2015). Therefore, we counted inflammatory cells in the bronchoalveolar lavage fluid (BALF), which mainly consisted of macrophages under specific pathogen-free conditions. Macrophages were significantly increased in miR449-compared to WT mice, although it was obviously and logically not as strong as in CSexposed WT mice (Figure 37b). To further study pulmonary inflammatory response upon miR449 deficiency, mice were treated with NTHi to boost inflammation (as described in Figure 34a). Upon NTHi treatment, protein levels of the M2A macrophage marker CD206 were elevated in lungs of miR449 ${ }^{--}$compared to WT mice (Figure $37 \mathrm{c}$ ). Accordingly, transcript levels of the COPD-associated matrix metalloproteinases Mmp9 (gelatinase) and Mmp12 (macrophage elastase), both expressed by macrophages (Houghton, 2015), were significantly upregulated in NTHi-treated miR449 ${ }^{-1}$ mice (Figure $37 \mathrm{~d}$ ). Conversely, the antiprotease level, as measured by the protein expression of tissue inhibitor of metalloproteinases 1 (TIMP1), remained unchanged upon miR449 depletion (Figure 37e and $\mathrm{f}$ ), thus it is not counteracting the observed increase in protease content.

Together, these results strongly suggest that $m i R 449^{-/}$mice develop spontaneous manifestations of COPD with age, which are associated with increased inflammatory response. 
a

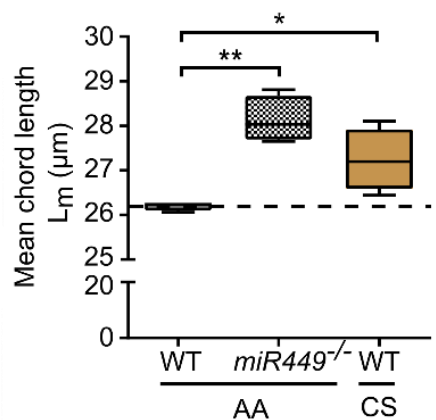

d

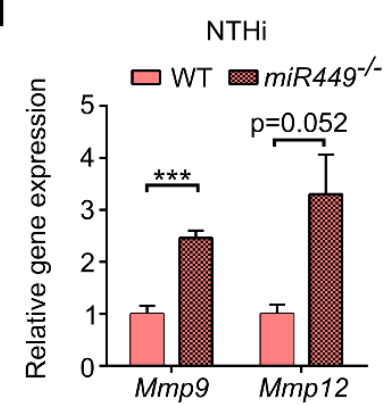

b

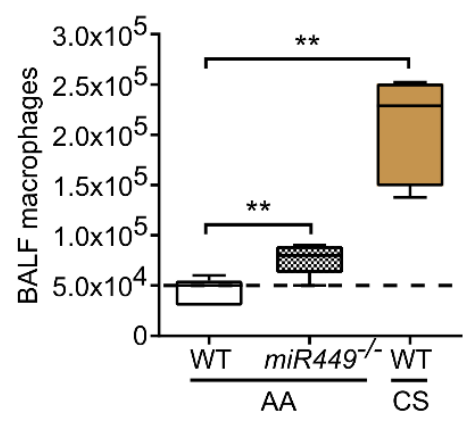

e

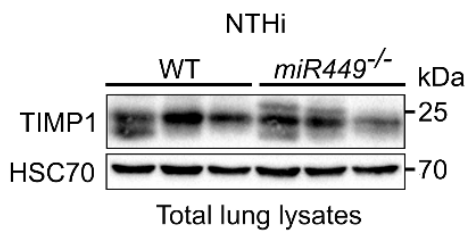

C

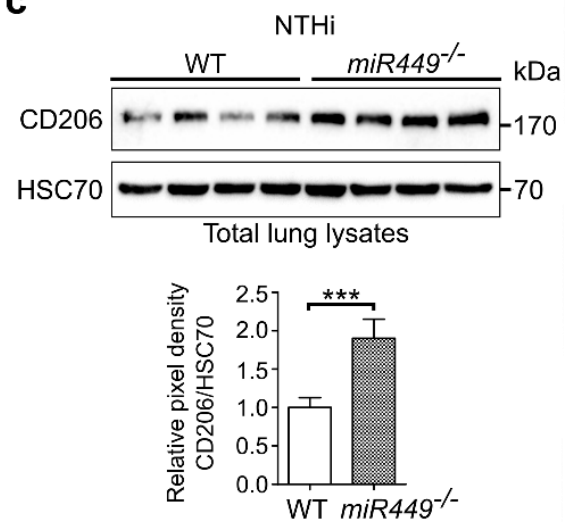

$\mathbf{f}$

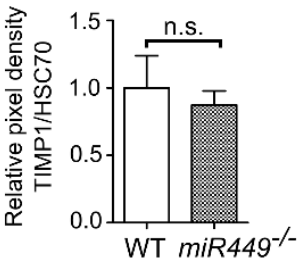

Figure 37: miR449-- mice develop spontaneous emphysema with increased inflammatory response. a) Emphysema was determined by measuring the mean chord length in 10 months old WT, miR449CS-exposed WT mice. $n=4$ mice/genotype. b) Macrophages were counted in BALF of 10 months old WT, miR449- , and CS-exposed WT mice. $n=5$ mice/genotype. c) CD206 levels, as marker for M2A macrophages, were analyzed by immunoblot in WT and miR449-- mice after NTHi treatment using HSC70 as a loading control. Graph shows quantification of immunoblot bands. WT serves as reference. d) Transcript levels of matrix metalloproteinases Mmp9 and Mmp12 in NTHi-treated WT and miR449-- mice normalized to 36b4. WT levels serve as reference. $n=4$ mice/genotype. e) TIMP1 levels were analyzed by immunoblot using HSC70 as a loading control. f) Quantification of immunoblot shown in (e). Data are presented as the mean \pm SEM.

\subsubsection{Aurora kinase $A$ is a key player in miR449-regulated ciliary homeostasis and epithelial regeneration processes}

Next, we aimed to elucidate the mechanism involved in the defective mucociliary regeneration in miR449 ${ }^{-/}$mice. Airway regeneration requires a balance between proliferation and differentiation to avoid pathologies and is coupled to ciliary disassembly and re-assembly (Beers and Morrisey, 2011; Goto et al., 2013). Due to a lack of convenient models for quantifying motile cilia assembly and disassembly, we studied the functions of miR449 in these key processes using a simplified in vitro model. Primary cilia assembly and disassembly can be monitored and measured on MEFs by serum withdrawal causing cell cycle exit and by subsequent serum addition leading to cell cycle re-entry, respectively

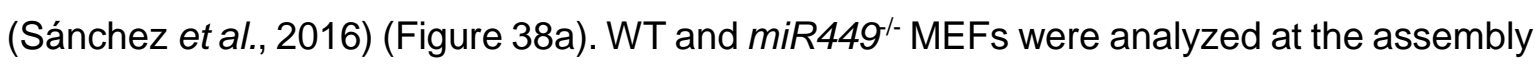
and disassembly time point by IF staining for cilia components of the axoneme (Ac- $\alpha$-TUB) and BB (Pericentrin). Surprisingly, there was no change in the assembly rate between the genotypes (Figure 38b and c). However, ciliary disassembly was significantly increased 
resulting in reduced ciliation in miR449-/ MEFs (Figure $38 \mathrm{~b}$ and c). High structural similarities between motile and primary cilia imply common mechanisms for ciliary assembly and disassembly (Satir and Christensen, 2007a). Hence, the increased primary cilia disassembly rate could explain the reduction in motile cilia in $m i R 449^{-/-}$mice.

a

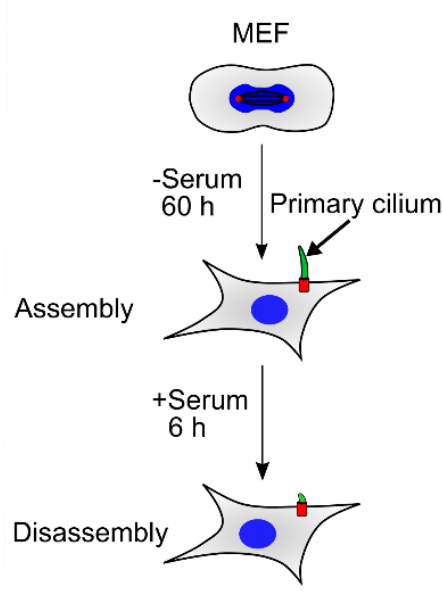

C

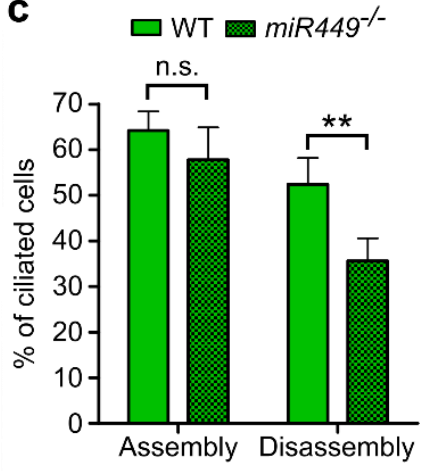

b
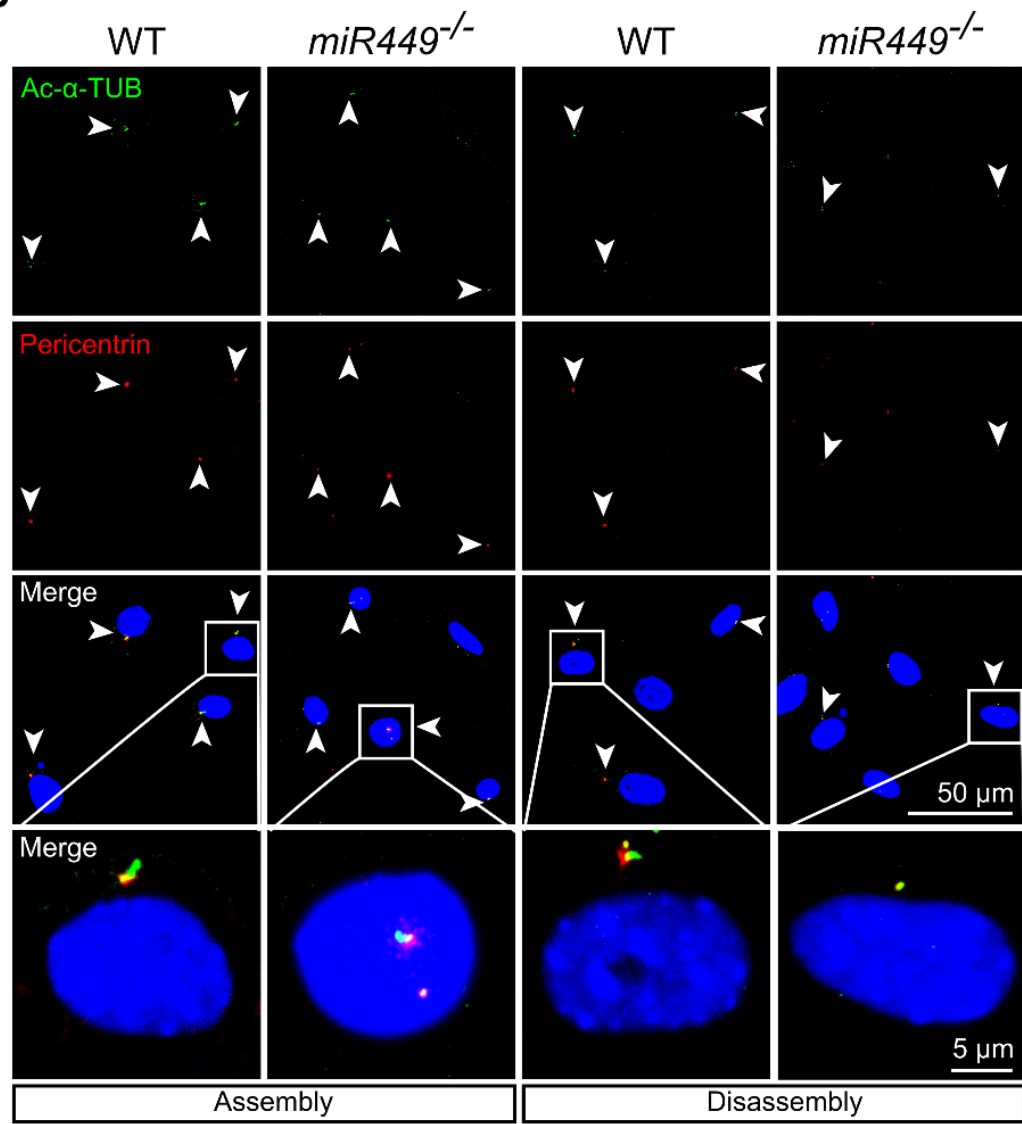

Figure 38: miR449 depletion increases primary cilia disassembly.

a) Schematic view of the experimental design for primary cilia assembly and disassembly in MEFs. Assembly of a primary cilium is induced by the removal of serum for $60 \mathrm{~h}$. Subsequent addition of serum for $6 \mathrm{~h}$ triggers ciliary disassembly. b) WT and miR449-- MEFs were stained with Ac-a-TUB (green) and Pericentrin (red) to visualize the ciliary axoneme and BB, respectively. Nuclei were counterstained with DAPI (blue). Arrowheads indicate primary cilia. Boxed regions are shown in higher magnification in the lowest panel for highlighting primary cilia. c) Quantification of the percentage of ciliated cells in WT and miR449- MEFs at the assembly and disassembly time point. Data are presented as the mean \pm SEM.

Given the increased disassembly rate in miR449-1 MEFs, we asked whether miR449 interferes with key players of the ciliary disassembly pathway (Goto et al., 2013; Sánchez et al., 2016) (Figure 39a). Trough analysis of published data (Lin et al., 2011b; Song et al., 2014; Mercey et al., 2017; Otto et al., 2017) and in silico prediction tools (TargetScan (Agarwal et al., 2015), PITA (Kertesz et al., 2007)), we identified human and murine AURKA, $H D A C 6$, and $K C N H 1$ as potential miR449 targets (Supplemental Figure 18a). Of note, when targeting several nodes within a pathway, it is likely that miRNAs elicit a profound effect on this pathway, since each individual miRNA target is typically only repressed to a modest 
degree (Baek et al., 2008). For detailed analysis, we focused on AURKA as a miR449 target, because of its pivotal role in HDAC6-mediated ciliary disassembly (Pan et al., 2004; Pugacheva et al., 2007; Lam et al., 2013), and its up-regulation in miR34 ${ }^{-/}$x miR449/- mice (Song et al., 2014; Otto et al., 2017). To verify the regulation of AURKA by miR449, we overexpressed miR449 in human lung carcinoma cells (H1299). Upon overexpression of miR449, protein as well as transcript levels of AURKA strongly declined (Figure 39b and c). In confirmation, AURKA levels were upregulated in lungs from aged and naphthalenetreated miR449 $^{-/}$mice compared to WT controls (Figure $39 \mathrm{~d}-\mathrm{f}$ ).

Taken together, these findings suggest that miR449 deficiency reduces airway ciliation by increasing the ciliary disassembly rate, probably through the upregulation of AURKA. 
a

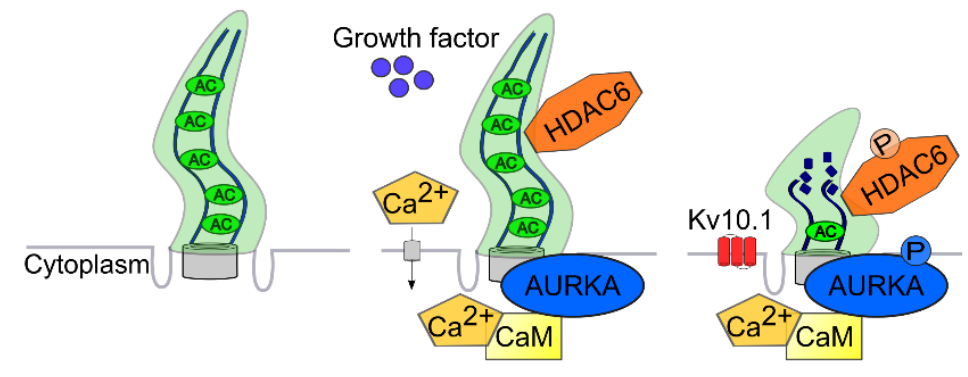

b

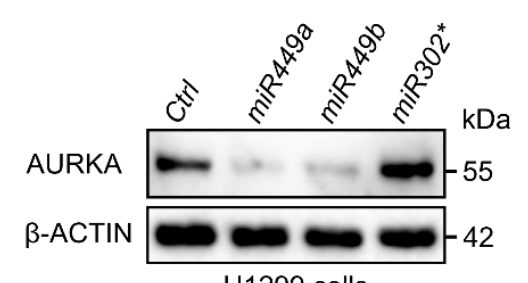

H1299 cells

C

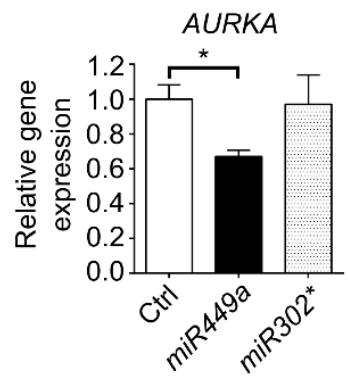

H1299 cells d

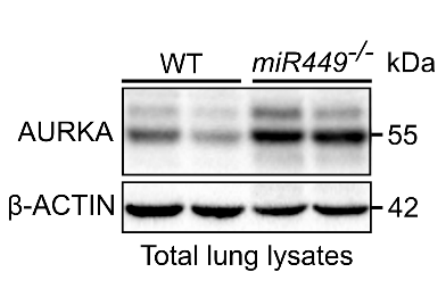

e

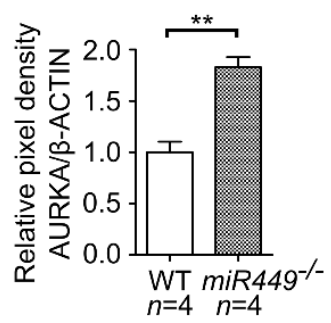

f

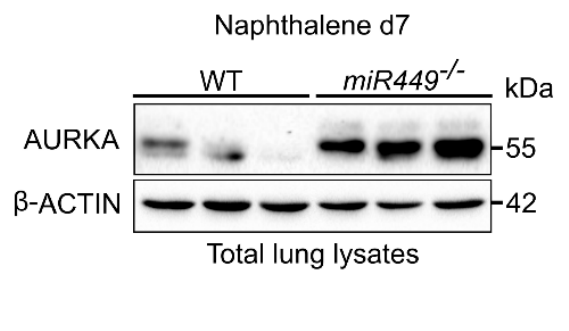

Figure 39: miR449 represses ciliary disassembly by targeting Aurora kinase A.

a) Schematic overview of the ciliary disassembly pathway (adapted from (Goto et al., 2013)]). Growth factor stimulation leads to $\mathrm{Ca}^{2+}$ influx into the cytoplasm. $\mathrm{Ca}^{2+}$ binds to $\mathrm{CaM}$, which subsequently binds to and activates AURKA by autophosphorylation. Activated AURKA phosphorylates HDAC6, which removes acetyl groups from a-tubulin and thereby triggering ciliary disassembly (Goto et al., 2013). Kv10.1 (KCNH1) promotes ciliary disassembly via an unknown mechanism (Sánchez et al., 2016). b and c) miR449a and miR449b pre-miRNAs were transfected into $\mathrm{H} 1299$ cells using a synthetic ctrl sequence (NC\#2) and a natural miRNA with no predicted binding site on AURKA (miR302*) as references. Lysates harvested at $48 \mathrm{~h}$ posttransfection were analyzed by immunoblot for AURKA protein levels (b) or by qPCR for transcript levels $(n=$ 3) (c). d) Immunoblot analysis of AURKA levels in lungs from 6 months old WT and miR449-- mice with $\beta$ ACTIN as loading control. e) Quantification of $n=4$ mice per genotype of AURKA immunoblot bands from aged WT and miR449-- mice. f) AURKA levels in lung lysates from naphthalene-treated WT and miR449-mice (d7) using $\beta$-ACTIN as a loading control. Data are presented as the mean \pm SEM. 


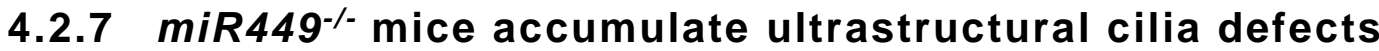 associated with reduced mucociliary clearance}

Increased ciliary disassembly caused by AURKA upregulation has been associated with primary cilia instability due to altered ultrastructure (Jacoby et al., 2009; Plotnikova et al., 2015). Indeed, electron microscopy revealed an altered ultrastructure of motile airway cilia characterized by an accumulation of ciliary plasma membrane detachments in tracheal sections from miR449/- mice (cilia with detachments are named abnormal cilia from here on), while the typical 9+2 arrangement of microtubules was maintained (Figure 40a and b). Defects in structural components of motile cilia impair the highly coordinated cilia beating process, resulting in mucociliary clearance disorders (Horani and Ferkol, 2016). Thus, we assumed that the observed abnormal airway cilia of $\mathrm{miR}_{4} 49^{-/}$mice would result in reduced mucociliary transport. To assess mucociliary transport, the velocity of fluorescently labelled beads over WT and miR449-/ tracheae was recorded, whereby only areas with beating cilia were analyzed. Indeed, bead transport was significantly diminished in miR449-/ mice (Figure 40c and d), indicating reduced clearance. Taken together, these results demonstrate that miR449 depletion impairs cilia structure and function.

Altogether, loss of miR449 in mice impairs bronchial epithelial regeneration upon environmental insults and aging, reduces airway cilia, and increases emphysematous and inflammatory manifestations, all consistent with a COPD phenotype. This is at least partially mediated by an upregulation of AURKA and increased ciliary disassembly and instability, ultimately resulting in mucociliary clearance defects in vivo (Figure 41). Our study reveals a new function of miR449 in regulating cilia maintenance and underlines the importance of balanced ciliated regeneration for respiratory health. 


\section{a}
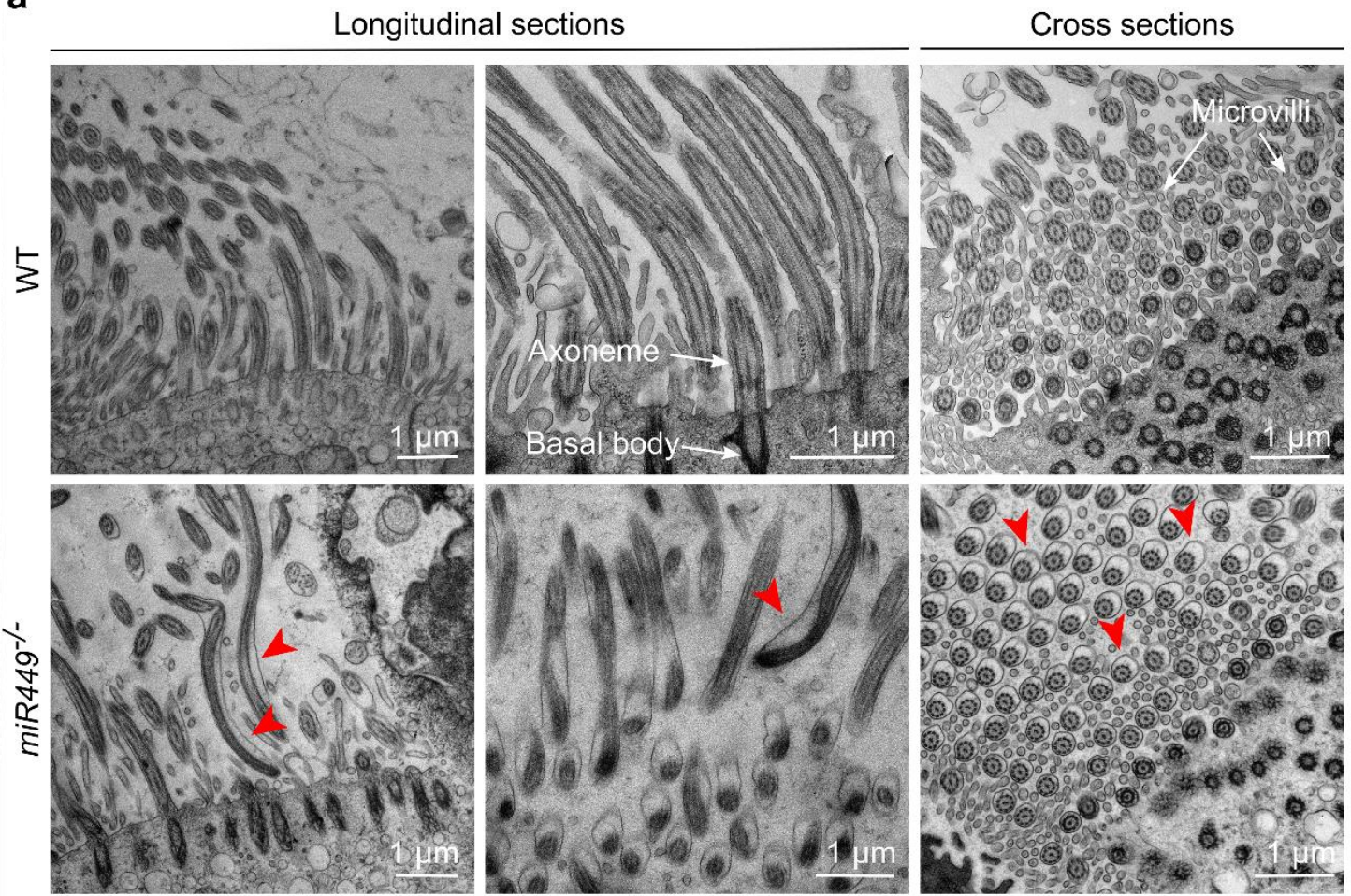

b

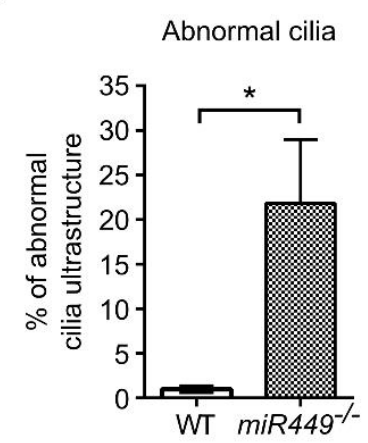

C

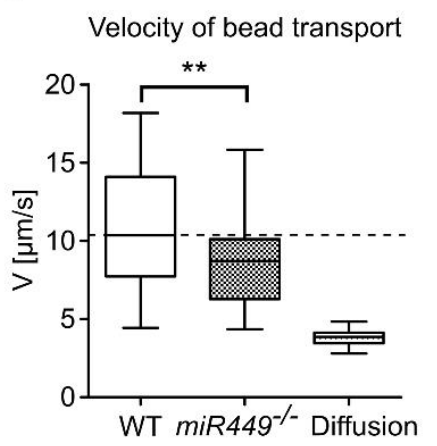

d

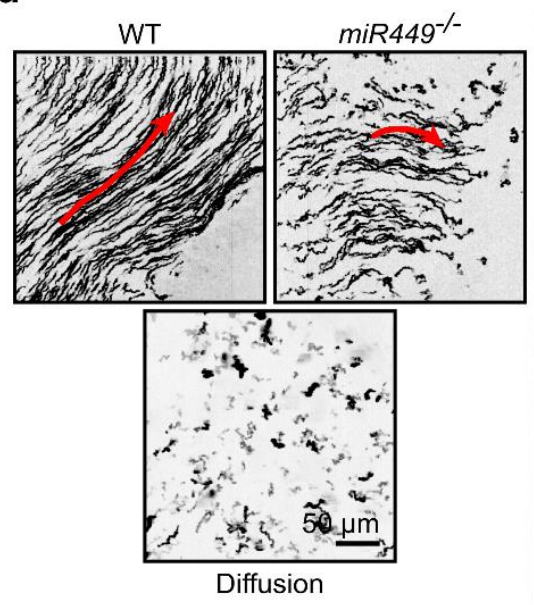

Figure 40: Loss of miR449 generates cilia ultrastructural defects accompanied by reduced mucociliary clearance.

a) Tracheae from WT and miR449-/ mice (7-9 weeks) were subjected to TEM. Cilia with atypical plasma membrane detachments ("abnormal cilia") are marked with red arrowheads. b) Quantification of the percentage of abnormal cilia found in TEM. $n=3$ WT (711 cilia from 168 images) and $n=5$ miR449- (305 cilia from 184 images). c) Bead transport velocity over WT and miR449-/ tracheae. Passive diffusion over dead tracheae of both genotypes serves as ctrl. $n=38$ WT measurements on 5 mice; $n=36$ miR449measurements on 5 mice; $n=25$ diffusion measurements on 9 mice. d) Bead trajectories aggregated from 2000 images over 32 seconds of movie. WTs display longer trajectories along a flow field. Red arrows indicate bead direction. Data are presented as the mean \pm SEM. 

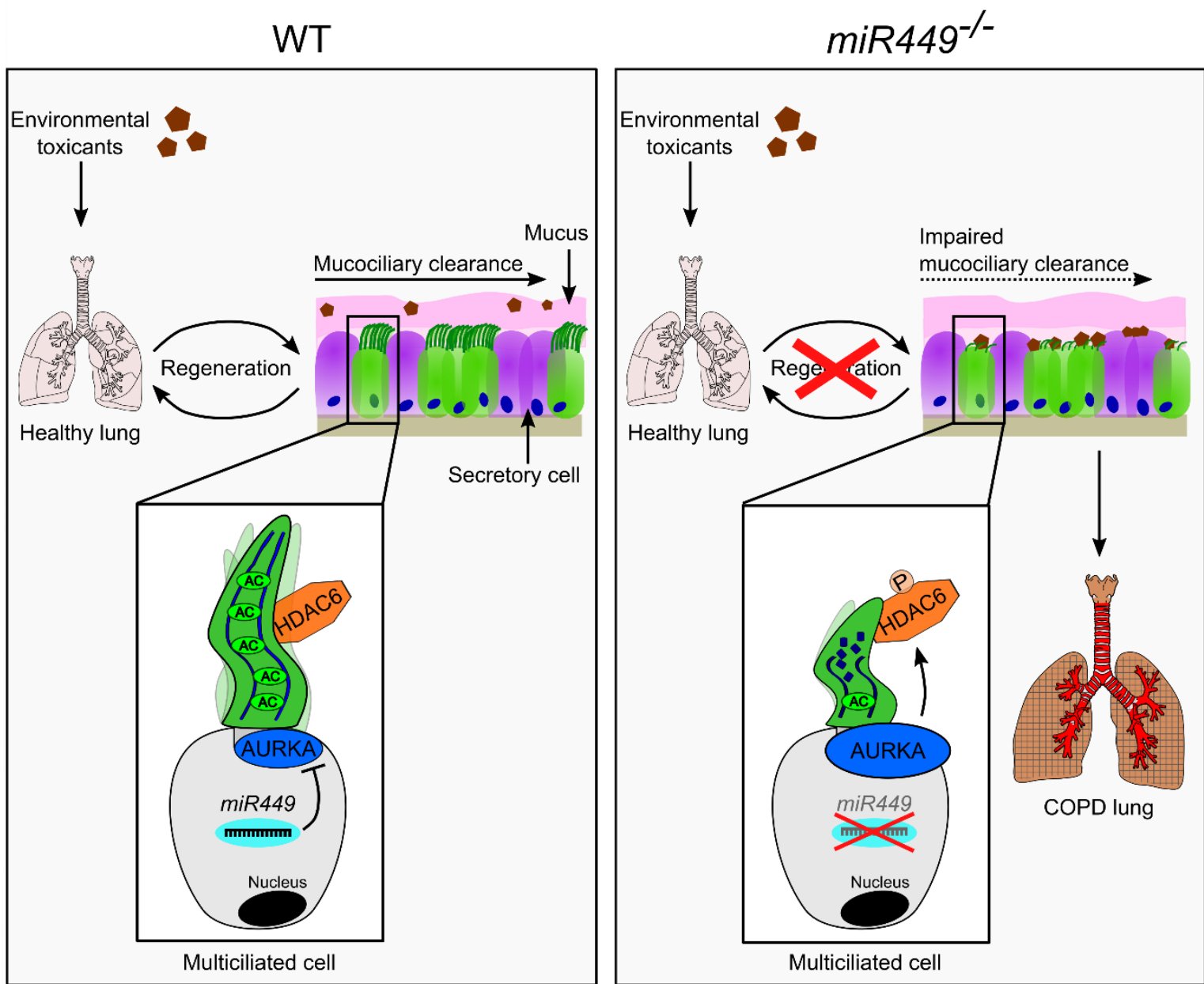

Figure 41: Proposed model of the protective role of miR449 in airway epithelial regeneration.

Left panel (WT mice): Upon reaching the airway epithelium, environmental toxicants (e.g. bacteria, CS, DEPs, naphthalene) trapped in mucus are transported out of the airways by coordinated beating of multiple motile cilia, a process termed mucociliary clearance. Efficient mucociliary clearance allows proper airway regeneration, thereby preventing chronic airway inflammation. miR449 prevents AURKA-mediated ciliary disassembly and thus, ensures cilia maintenance, which is required for proper mucociliary clearance. Right panel (miR449--mice): Loss of miR449 impairs ciliated epithelial regeneration and mucociliary clearance by increasing AURKA-mediated ciliary disassembly. Thus, in miR449-/ mice environmental toxicants are not cleared from the airways resulting in increased pulmonary inflammation and development of emphysematous manifestations of COPD. 


\subsubsection{Supplemental Data}

\section{Supplemental Tables}

Supplemental Table 1: Characteristics of COPD patients enrolled in the GLUCOLD study.

\section{Characteristic}

Gender (male), n (\%)

Ex-smokers, n (\%)

Pack-year ${ }^{*}$

Age, year ${ }^{*}$

FEV $_{1}, \%$ predicted

$\mathrm{FEV}_{1} / \mathrm{FVC}^{*}$

*Data are presented as median (interquartile range); FEV1 is forced expiratory volume in 1 second;

\section{COPD patients $(n=57)$}

48 (84.2)

$35(61.4)$

41.5 (31.9-53.3)

59 (54-66)

$64.3(58.5-69.8)$

$0.50(0.44-0.56)$

Supplemental Table 2: Top 10 enriched biological processes among miR34a-5p-positivly correlated genes in COPD patients.

\section{NAME}

DNA_DAMAGE_RESPONSE_SIGNAL_TRANSDUCTION_RESULT ING_IN_TRANSCRIPTION

EPOXYGENASE_P450_PATHWAY

\begin{tabular}{|c|c|c|}
\hline AXONEMAL_DYNEIN_COMPLEX_ASSEMBLY* & 0,0094 & 0,8557 \\
\hline MICROTUBULE_BUNDLE_FORMATION* & 0,0018 & 0,6606 \\
\hline GLYCERALDEHYDE_3_PHOSPHATE_METABOLIC_PROCESS & 0,0020 & 0,5371 \\
\hline KERATINIZATION & 0,0036 & 0,4565 \\
\hline AXONEME_ASSEMBLY* & $<0.001$ & 0,3979 \\
\hline GLUCOSE_6_PHOSPHATE_METABOLIC_PROCESS & 0,0057 & 0,4535 \\
\hline HISTONE_H3_DEACETYLATION & 0,0054 & 0,4680 \\
\hline MORPHOGENESIS_OF_A_POLARIZED_EPITHELIUM & 0,0018 & 0,4294 \\
\hline
\end{tabular}

${ }^{*}$ Cilia-associated processes are highlighted in green; GSEA was performed using the list of genes ranked based on the strength of their correlation with miR34a-5p and the Gene Ontology dataset for biological processes.

Supplemental Table 3: Top 10 enriched biological processes among miR34b-5p-positivly correlated genes in COPD patients.

\section{NAME}

CILIUM_MORPHOGENESIS*

NOM p-val FDR q-val

CILIUM_ORGANIZATION*

$<0.001$

$<0.001$

CELLULAR_COMPONENT_ASSEMBLY_INVOLVED_IN_MORPHO GENESIS

CELL_PROJECTION_ASSEMBLY

$<0.001$

$<0.001$

AXONEME_ASSEMBLY*

$<0.001 \quad<0.001$

CILIUM_MOVEMENT*

$<0.001<0.001$

MICROTUBULE_BUNDLE_FORMATION*

$<0.001<0.001$

$<0.001$

$<0.001$

MICROTUBULE_BASED_MOVEMENT*

$<0.001<0.001$

ORGANELLE_ASSEMBLY

$<0.001$

$<0.001$

PROTEIN_TRANSPORT_ALONG_MICROTUBULE*

$<0.001$

$<0.001$

${ }^{*}$ Cilia-associated processes are highlighted in green; GSEA was performed using the list of genes ranked based on the strength of their correlation with miR34b-5p and the Gene Ontology dataset for biological processes. 
Supplemental Table 4: Top 10 enriched biological processes for miR34c-5p-positivly correlated genes in COPD patients.

\begin{tabular}{|l|l|l|}
\hline NAME & NOM p-val & FDR q-val \\
\hline CILIUM_ORGANIZATION* & $<0.001$ & $<0.001$ \\
CILIUM_MORPHOGENESIS* $^{*}$ & $<0.001$ & $<0.001$ \\
\hline CELLULAR_COMPONENT_ASSEMBLY_INVOLVED_IN_MORPHOGENESIS & $<0.001$ & $<0.001$ \\
\hline CELL_PROJECTION_ASSEMBLY & $<0.001$ & $<0.001$ \\
\hline AXONEME_ASSEMBLY* & $<0.001$ & $<0.001$ \\
CILIUM_MOVEMENT* $^{*}$ & $<0.001$ & $<0.001$ \\
MICROTUBULE_BUNDLE_FORMATION* $^{*}$ & $<0.001$ & $<0.001$ \\
\hline MICROTUBULE_BASED_MOVEMENT* & $<0.001$ & $<0.001$ \\
\hline ORGANELLE_ASSEMBLY & $<0.001$ & $<0.001$ \\
\hline PROTEIN_TRANSPORT_ALONG_MICROTUBULE* & $<0.001$ & 00.001 \\
\hline
\end{tabular}

*Cilia-associated processes are highlighted in green; GSEA was performed using the list of genes ranked based on the strength of their correlation with miR34b-5p and the Gene Ontology dataset for biological processes.

\section{Supplemental Figures}

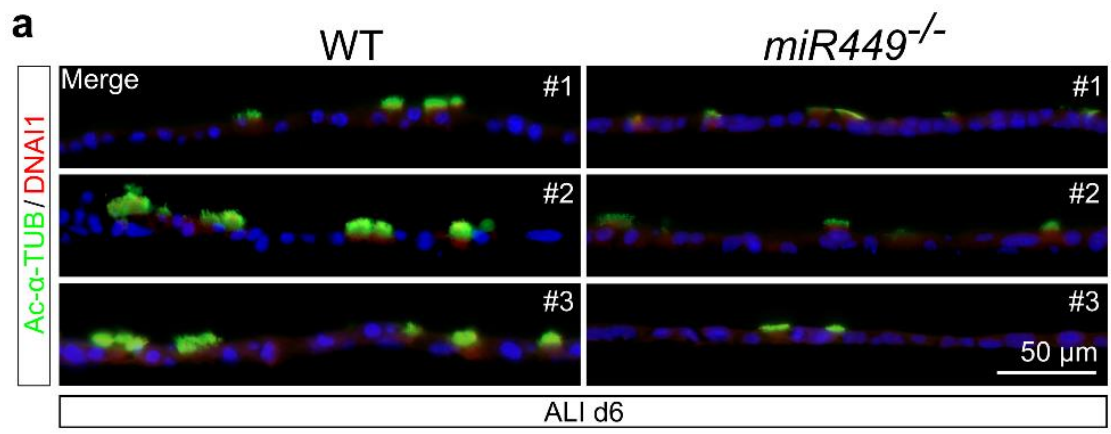

Supplemental Figure 14: miR449\% ALI cultures express less cilia markers than their WT counterparts.

a) IF staining of axonemal cilia markers Ac-a-TUB (green) and DNAI1 (red) in WT and miR449-- ALI cultures at d6. Nuclei were counterstained with DAPI (blue). Images from $n=3 \mathrm{ALI}$ cultures per genotype. For one ALI culture 3 mice were used. 
a

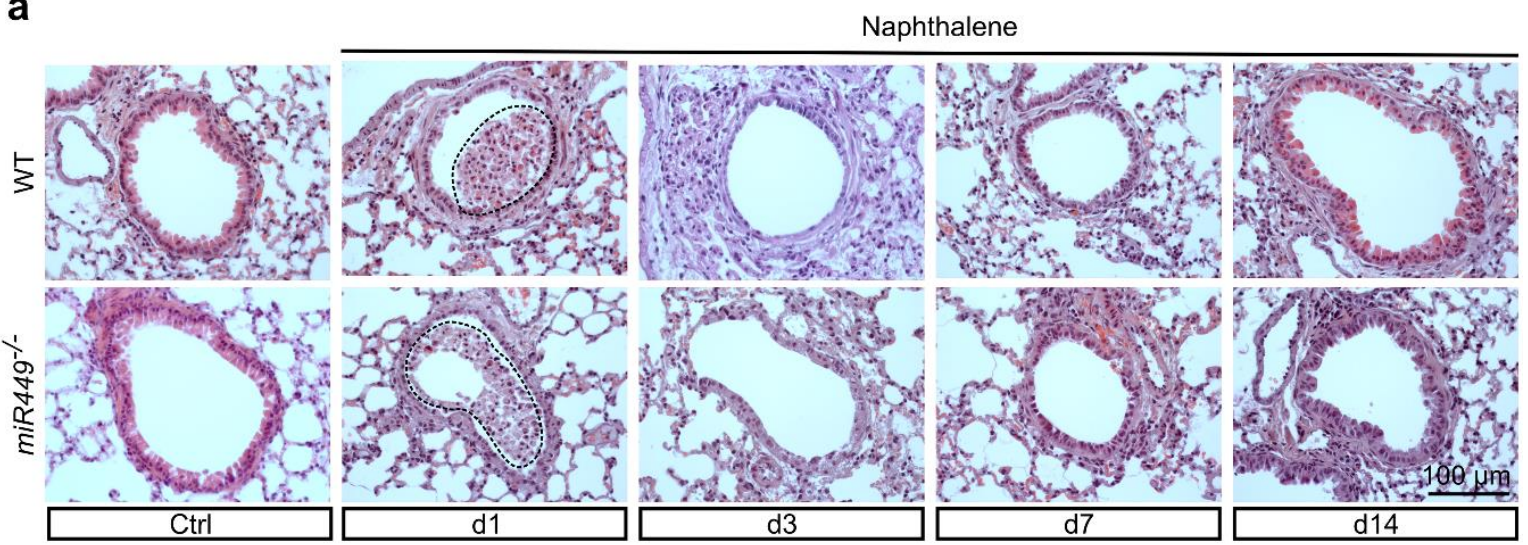

b Naphthalene

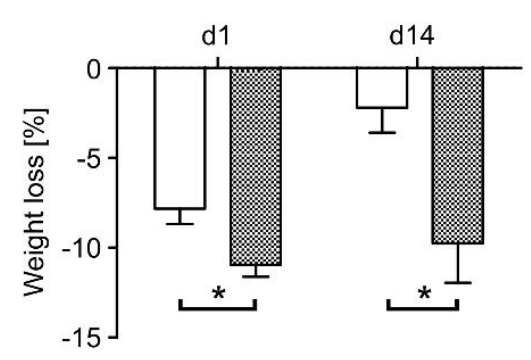

$\square$ WT

miR449\%

\section{Supplemental Figure 15: Naphthalene treatment affects lung histology in WT and miR449-/ mice to} a similar extent.

a) H\&E staining of lung sections from oil (ctrl) and naphthalene treated WT and miR449-- mice. Ctrl mice (oil) show a pseudostratified bronchial epithelium. After naphthalene injection at d1, injured club cells exfoliate and accumulate in the airway lumen. Exfoliated club cells are marked by a dotted circle at d1. b) Weight of WT and miR449-\% mice was recorded before naphthalene injection (d0) and at different time points after injection (d1 and d14). Weight loss [\%] represents the difference between the weight before and after naphthalene injection. WT: $n=10$ (d1) and $n=5$ (d14) mice per group; miR449 $\cdots: n=9$ (d1), and $n=4$ (d14) mice per group. Data are presented as the mean \pm SEM.

a

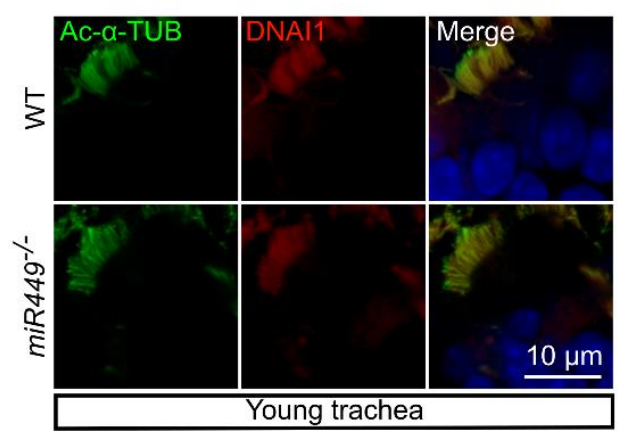

b

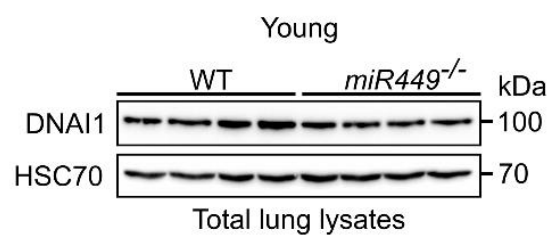

Supplemental Figure 16: Ciliation appears normal in young $m i R 449 \%$ mice.

a) Cryosections of 10 weeks old WT and miR449- trachea were stained with Ac-a-TUB (green) and DNAl1 (red). Nuclei were counterstained with DAPI (blue). Images were taken with a confocal microscope. b) Lung lysates from 10 weeks old WT and miR449-- were analyzed by immunoblot using DNAl1 as a motile cilia marker and the constitutive protein HSC70 as a loading control. 

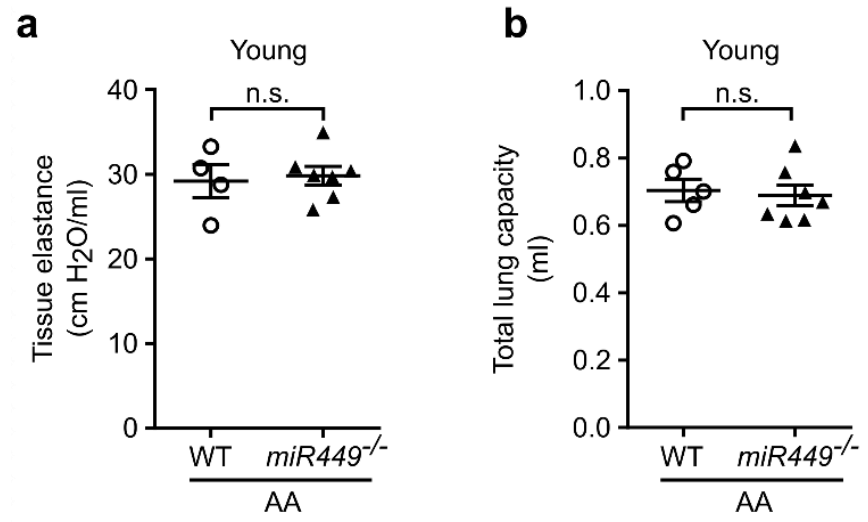

Supplemental Figure 17: Young miR449-/ mice do not display any signs of spontaneous COPD. a and b) Pulmonary function was assessed in 12 weeks old WT and miR449-- mice exposed to AA by measuring the tissue elastance (a) and total lung capacity (b) using the FlexiVent system. $n=4-7$ mice/genotype. Data are presented as the mean \pm SEM.

a

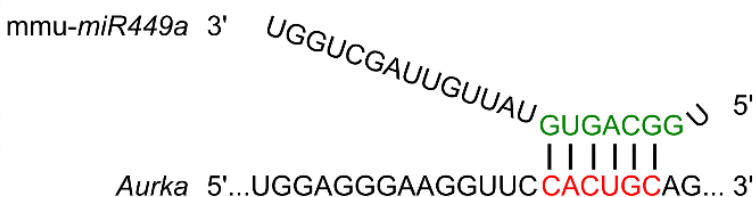

mmu-miR449a $3^{\prime}$

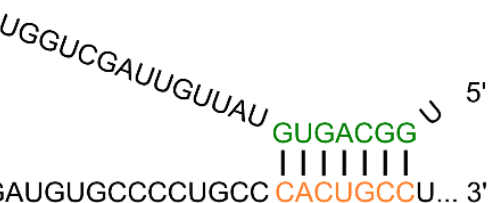

mmu-miR449a $3^{\prime}$

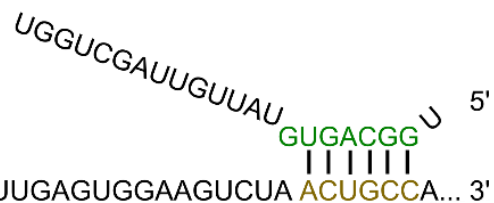

Hdac6 $5^{\prime}$...GAUGUGCCCCUGCC CACUGCCU... 3'

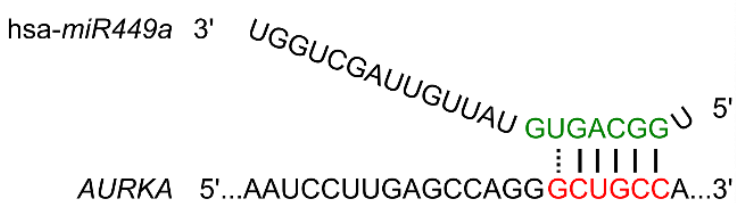

hsa-miR449a

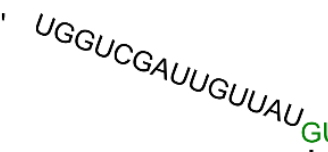

IIIIII

HDAC6 $55^{\prime} . . . A G G G G U A G C C U C C$ CACUGCAU... $3^{\prime}$

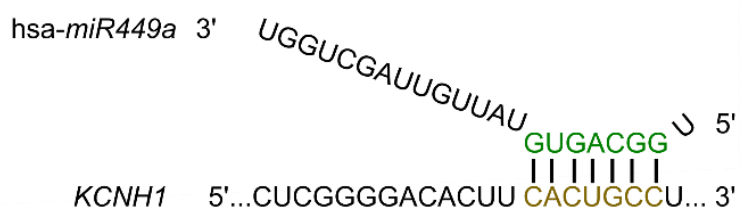

Supplemental Figure 18: Predicted targets involved in ciliary disassembly of miR449.

a) Mouse and human AURKA (Otto et al., 2017), HDAC6 (Song et al., 2014), as well as KCNH1 (Lin et al., 2011b) possess binding sites for miR449 in their 3'UTR. The seed sequence of miR449a is shown in green. 


\section{DISCUSSION}

Proper beating of multiple motile cilia is of great importance for human health. Dysfunction of motile cilia can cause infertility, hydrocephalus, and defective mucociliary clearance, which results in respiratory infections and lung injury (Spassky and Meunier, 2017). However, the regulation of the highly complex network of multiciliogenesis is poorly understood. It is necessary to understand the program of multiciliogenesis to develop new therapeutic approaches for the treatment of inherited and acquired cilia dysfunctions. In this thesis, we aimed to evaluate the role of two different multiciliogenesis regulators: the transcription factor and tumor suppressor TAp73 and the post-transcriptional regulator miR449 in various multiciliated tissues. To achieve this, we analyzed different multiciliated epithelia from mice depleted for TAp73, miR449, or both. Here, we demonstrate that TAp73 as well as miR449 are key regulators of the complex hierarchy of multiciliogenesis by promoting it at different steps. TAp73, on one hand, is required for the generation of multiciliated epithelia in the female and male reproductive tract by activating the transcription of several pro-ciliogenic factors. This is in line with a previous report from us and another group showing that TAp73 is a central regulator of airway multiciliogenesis (Marshall et al., 2016; Nemajerova et al., 2016). Interestingly, our study illustrates that multiciliogenesis is differently regulated within multiciliated tissues. miR449, on the other hand, promotes the maintenance of motile cilia and thereby facilitates proper regeneration of the multiciliated airway epithelium, which is required for healthy lung aging.

\subsection{TAp73 - Tissue-dependent regulation of multiciliogenesis}

So far, the role of TAp73 has been extensively described in airway multiciliogenesis (Nemajerova et al., 2016). Briefly, TAp73 depletion causes loss of airway cilia accompanied by impaired mucociliary clearance (Figure $12 \mathrm{~g}$ - j). TAp73 ensures airway multiciliogenesis by directly activating the transcription of several key players of multiciliogenesis such as Foxj1, Rfx2, Rfx3, Myb, miR34b,c, and structural and functional ciliary genes (Figure 13) (Marshall et al., 2016; Nemajerova et al., 2016). In our study, we describe the role of TAp73 in further multiciliated tissues (Figure $27 \mathrm{a}-\mathrm{d}$ ). We observed that the depletion of TAp73 resulted in defective multiciliogenesis in EDs (Figure 16a - d, Figure 17a - c) and FTs (Figure $18 \mathrm{a}-\mathrm{d}$, Figure 19a and b) in mice. Altogether, these data indicate that TAp73 is key regulator of multiciliogenesis in airways and reproductive tracts. However, TAp73 is dispensable for the ciliary function in the ventricles of the brain (Figure 21a - c, Figure 22a and $b$ ), indicating that the regulatory network of multiciliogenesis is more robust in the ventricles compared to other multiciliated tissues. Similar to this observation, it has been shown that depletion of miR-34/449 results in a severe loss of motile cilia in all murine 
multiciliated organs except for the ventricles as hydrocephalus was not detected (Song et al., 2014; Wu et al., 2014; Otto et al., 2017). Moreover, Danielian and colleagues demonstrated that E2F4 is required for the development of the ciliated epithelium in the airways but not for the ciliated epithelium of the FTs, EDs, and ventricles (Danielian et al., 2007). To summarize, these data illustrate that the regulation of multiciliogenesis is tissuedependent. The remaining question is what makes the ventricles of the brain different. This question will be further discussed in the section 5.1.3.1.

\subsubsection{Role of motile cilia in causing male infertility}

Male fertility is mainly depended on three processes: 1) Spermatogenesis resulting in functional spermatozoa. 2) Transport of immotile spermatozoa from their production site (testis) to their storage/maturation place (epididymis) via the EDs, and 3) Spermatozoa concentration in the EDs achieved by testicular fluid reabsorption.

It has been shown that male TAp73 KO mice are infertile due to defective spermatogenesis (Tomasini et al., 2008). More precisely, loss of TAp73, which is specifically expressed in germ cells (Hamer et al., 2001; Inoue et al., 2014), resulted in impaired germ cell adhesion and maintenance, and spermatozoa maturation (Holembowski et al., 2014; Inoue et al., 2014). Consequently, this led to a strong decline in the number of spermatozoa, which resulted in a "near-empty seminiferous tuble" phenotype in the testis of TAp73 KO mice (Holembowski et al., 2014). This is similar to the effect we observed in male testis (Supplemental Figure 2a). Of note, some seminiferous tubes were filled with spermatozoa. However, we did not detect any spermatozoa in the cauda epididymis (Figure 16a), indicative of a defect in the conduit (EDs) connecting the testis and the epididymis. Here, we provide interesting new data regarding this issue as TAp73 loss causes defects in the multiciliated epithelium of the EDs by reducing the number and length of ED cilia (Figure $16 \mathrm{~b}$ - d). Thus, TAp73 does not only regulate multiciliogenesis in the airways (Nemajerova et al., 2016), but also in the EDs.

The function of motile cilia in the EDs is not well described. It is assumed that motile cilia in the EDs possess a rotational beating pattern (llio and Hess, 1994; Hess, 2002). The rotational beating pattern is a unique feature of cilia in the EDs as motile cilia of other multiciliated epithelia possess a back and forth beating pattern (Satir et al., 2014). The coordinated back and forth beating pattern of these motile cilia (in FT, airway, brain ventricles) is responsible for the directed transport of fluid containing particles/gametes/zygotes (Spassky and Meunier, 2017). However, the rotational beating of ED cilia prevents the coordinated transport of spermatozoa towards the epididymis (Hess, 2015). Thus, it is hypothesized that motile cilia in the EDs stir the testicular fluid and thereby facilitating fluid circulation, reabsorption, and spermatozoa concentration. Each of 
these processes represent an important parameter that determines effective spermatozoa transport through the EDs (Hess, 2015). One finding supporting the "rotational beating pattern" hypothesis of ED cilia is based on ultrastructural analysis of ED cilia, which showed twisted microtubules at the ciliary tip. These twisted microtubules are only observed in ED cilia and thus, might represent an explanation for the distinct beating pattern (Hess, 2015). However, the rotational beating pattern is only mentioned in some reports lacking video evidence (llio and Hess, 1994; Hess, 2015). Hence, further video evidence would be required to proof such rotational beating pattern of ED cilia.

How are immotile spermatozoa transported through the EDs, if not by the beating of motile cilia? It is suggested that spermatozoa transport via the EDs depends on the interaction of several forces including smooth muscle contraction and fluid dynamics (Hess, 2002). We ruled out that TAp73 loss negatively impacts smooth muscle contraction since smooth muscle contraction is not altered in FTs from TAp73 KO mice (video recorded by us, unpublished). Moreover, TAp73 is only expressed in multiciliated cells and germ cells (Supplemental Figure 1a) (Hamer et al., 2001; Holembowski et al., 2014; Inoue et al., 2014), but not in smooth muscle cells. However, it is possible that TAp73 loss influences fluid dynamics. Up to $90 \%$ of the seminiferous fluid produced in the testis is reabsorbed in the EDs to concentrate spermatozoa, which is required for effective spermatozoa transport (Figure 42a) (Ilio and Hess, 1994; Yeste et al., 2017). Impaired fluid reabsorption in the EDs has been shown to contribute to male infertility (Zhou et al., 2001; Mendive et al., 2006; Danielian et al., 2016), highlighting the importance of tightly balanced fluid dynamics. Water reabsorption occurs via aquaporin channels, which are not only expressed on microvilli of nonciliated cells but also on cilia of ciliated cells in the EDs (Ruz et al., 2006; Yeste et al., 2017). Consequently, the reduced number of motile cilia in EDs of TAp73 KO mice might lead to a decline in the expression of aquaporin channels and defective seminiferous fluid reabsorption, thereby resulting in fluid accumulation (Figure 42b). In addition, impaired fluid reabsorption in the EDs can cause reflow of the fluid, which results in the dilation of the rete testis (Zhou et al., 2001; Mendive et al., 2006). Hence, to validate the effects of TAp73 loss on fluid reabsorption, it is necessary to study whether TAp73 KO mice express less aquaporin channels (e.g. AQP 1) and display dilated rete testis. Aquaporin channel expression can be quantified by IF staining on ED sections or by WB analysis of ED protein lysates. Rete testis dilation can be assessed by histological analysis of paraffin-embedded testes. 

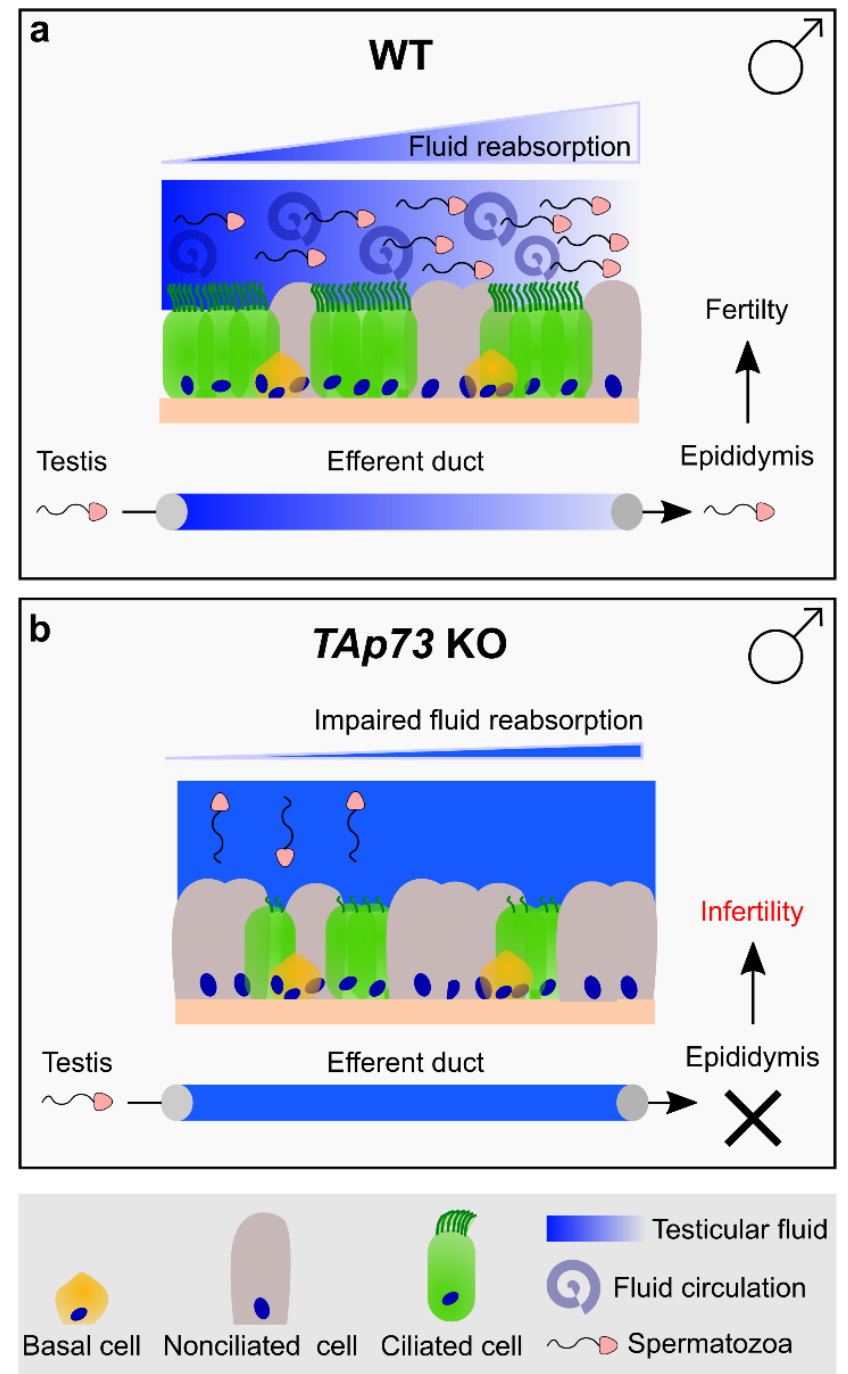

Figure 42: Proposed mechanism leading to male infertility in TAp73 KO mice.

a) Spermatozoa produced in the testis are transported via the EDs to the epididymis in fertile male WT mice. Ciliated cells of the EDs contribute to the spermatozoa transport by facilitating testicular fluid circulation, fluid reabsorption, and spermatozoa concentration. b) Number and length of motile cilia in EDs are strongly reduced in male TAp73 KO mice. Defective ED cilia might impair spermatozoa transport by declining fluid circulation, fluid reabsorption, and spermatozoa concentration. Due to the defective transport, spermatozoa do not reach the epididymis and thereby contributing to male infertility in addition to reduced spermatozoa production.

Altogether, current knowledge suggests that motile cilia of the EDs ensure male fertility by regulating testicular fluid circulation, fluid homeostasis, and spermatozoa concentration (Hess, 2015; Yeste et al., 2017). Thus, our data indicates that defective multiciliogenesis in EDs contributes to male infertility in TAp73 KO mice by disrupting testicular fluid dynamics (Figure 42a and b).

The link between cilia dysfunction, impaired fluid dynamics, and male fertility is supported by another study investigating the effect of the depletion of the pro-ciliogenic transcription factors E2f4 and E2f5 on male fertility (Danielian et al., 2016), which activate the transcription of TAp73 (Nemajerova et al., 2016). Mice harboring a specific depletion of $E 2 f 4 / 5$ (E2f4 ${ }^{-1+}$; $E 2 f 5^{-/}$; Vil-cre KO mice; E2f4/5 KO form here on) in the ED epithelium 
showed a similar phenotype as the one we observed in TAp73 KO mice (Figure 16a -d). Briefly, male E2f4/5 KO mice are infertile and showed a loss of ED cilia, no spermatozoa in the epididymis, testicular fluid accumulation, and dilation of the rete testis. Of note, the last two alterations were not studied in TAp73 KO mice. The phenotypic signs of E2f4/5 KO mice suggest that impaired ciliogenesis in the EDs compromises fluid reabsorption, thereby leading to male infertility. However, E2F4/5 also impairs the ability of nonciliated cells in the ED to reabsorb the fluid as E2F4/5 is not only expressed in ciliated but also in nonciliated cells. Thus, it is unclear whether the underlying mechanism of male infertility in E2f4/5 KO mice is based on defective ciliated or nonciliated cells (Danielian et al., 2016). As TAp73 is specifically expressed in ciliated cells and not in nonciliated cells in the ED epithelium (Supplemental Figure 1a), our study provides new insights into the connection between ED cilia and male infertility.

Although male infertility occurs in genetically modified mice depleted for factors involved in the regulation of multiciliogenesis (e.g. miR-34/449, Foxj1, Gemc1), the underlying mechanism is either not known or is attributed to dysfunctional spermatogenesis (Chen et al., 1998; Comazzetto et al., 2014; Wu et al., 2014; Yuan et al., 2015; Terré et al., 2016). For example, previous studies have connected male infertility in miR-34/449 KO mice to impaired spermatogenesis (Bao et al., 2012b; Comazzetto et al., 2014; Wu et al., 2014; Yuan et al., 2015) as miR-34/449, like TAp73 (Hamer et al., 2001; Holembowski et al., 2014; Inoue et al., 2014), are also expressed in germ cells (Bao et al., 2012b). However, they did not investigate the ciliation of the ED epithelium in miR-34/449 KO mice. As miR-34/449 is necessary for proper multiciliogenesis in airways and FTs (Song et al., 2014; Otto et al., 2017), it is likely that the ciliated epithelium of the ED could also be affected and contribute to the infertility phenotype in miR-34/449 KO mice.

To conclude, studies including ours indicate that functional motile cilia in the EDs are required for male fertility. However, to find out whether dysfunction of motile cilia in the ED epithelium is sufficient to cause male infertility irrespective of defective spermatogenesis, a specific depletion of pro-ciliogenic factors (e.g. TAp73) in multiciliated cells is required. This can be achieved by combining cell-type-specific gene promotors (e.g. Foxj1 promotor) with the Cre/LoxP system (Ostrowski et al., 2003; Zhang et al., 2007).

\subsubsection{Role of multiciliogenesis in ensuring female fertility}

Female infertility of TAp73 KO mice has been associated with poor oocyte quality (Tomasini et al., 2008; Tomasini et al., 2009). Interestingly, it was reported that ovulated oocytes are trapped in the bursa of TAp73 KO mice (Tomasini et al., 2008). Oocytes are usually captured by motile cilia of the fimbriae and then transported along the epithelium of the FT by coordinated beating of multiple motile cilia (Figure 43a) (Ezzati et al., 2014). Here, we 
show that deficiency of TAp73 reduces the number of cilia in FTs (Figure 18a - d) and thus, impairs oocytes capture and transport through the FT. Consequently, lack of cilia-driven transport along the epithelium of the FT of TAp73 KO mice might contribute to female infertility in these mice (Figure 43b).
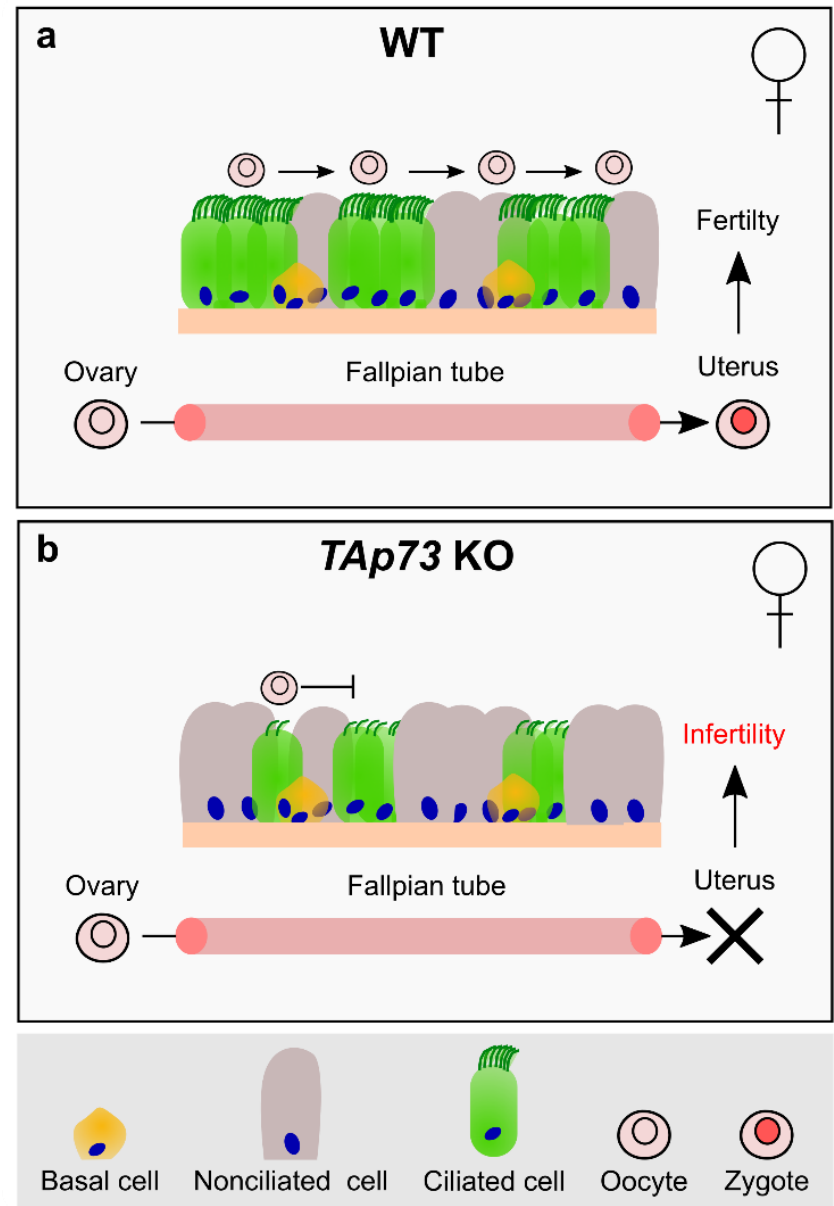

Figure 43: Proposed mechanism leading to female infertility in TAp73 KO mice.

a) In fertile WT mice, oocytes are released from the ovary, captured by ciliated fimbriae, and transported by coordinated beating of multiple motile cilia through the FT. Upon fertilization in the distal part of the FT, implantation of the fertilized egg (zygote) takes place in the uterus. b) TAp73 KO mice possess less motile cilia resulting in impaired oocytes capture and transport along the epithelium of the FT. Consequently, oocytes do not reach the distal part of the FT so that fertilization can no longer take place, resulting in infertility.

Remarkably, two single nucleotide polymorphisms (SNPs) in TAp73 were found in patients receiving in vitro fertilization, however the functional consequence of these SNPs is not known (Feng et al., 2011; Hu et al., 2011). Furthermore, TAp73 is downregulated in aged women, when motile cilia are not required anymore for gamete/zygote transport in the FT (Guglielmino et al., 2011). Together, these patient data highlight the importance of TAp73 in ensuring female fertility.

The importance of cilia-driven transport in ensuring female fertility has been reported by other studies. Terre and colleagues analyzed the ciliation of mice deficient for Gemc1, a 
regulator of multiciliogenesis upstream of TAp73 (Figure 13). They observed that female Gemc1 KO mice lack motile cilia in the FTs and are infertile (Terré et al., 2016). Similar results have been obtained by depleting other pro-ciliogenic factors of multiciliogenesis such as Ccno and miR-34/449 (Núnez-Ollé et al., 2017; Otto et al., 2017). Interestingly, Ccno mutations have been associated with increased infertility in women, which suffer from the syndrome called reduced generation of multiple motile cilia (Amirav et al., 2016). Moreover, defects in ciliary functions have been associated with ectopic pregnancies or infertility (Lyons et al., 2006; Vanaken et al., 2017).

In summary, we show that TAp73 is important for multiciliogenesis in the male and female reproductive tract. Hence, we hypothesize that dysfunction of motile cilia in FTs and EDs contributes to the infertility phenotype in TAp73 KO mice. However, further investigation using cell-specific deletion of TAp73 is required to form a direct link between dysfunction of motile cilia and infertility.

\subsubsection{Transcriptional regulation of multiciliogenesis in the ventricles of the brain - An exception}

Depletion of TAp73 neither affects the number and functionality of motile ependymal cilia nor the expression of pro-ciliogenic factors (e.g. Foxj1, Rfx2, and $R f x 3$ ) in the ventricles of the brain (Figure 21b and c, Figure 22a and b, Supplemental Figure 5b - d). Moreover, we and others have observed that TAp73 KO mice do not develop a hydrocephalus (Tomassini 2008) (Figure 25a, Supplemental Figure 9a), which would have normally occurred as a consequence of cilia dysfunction (Ibañez-Tallon et al., 2002; Ibanez-Tallon, 2004; Lee, 2013; Ohata et al., 2014). Thus, TAp73 seems to be dispensable for the development and maintenance of functional motile cilia on ependymal cells. This is in line with a recent report showing that conditional deletion of $p 73$ (deletion of both TAp73 and DNp73) in adult mice (conditional p73 KO mice: p73flox/flox;Foxj1CreERT;R26R-tdTomato) did not cause cilia deficiency in the ventricles of the brain and hydrocephalus (Fujitani et al., 2017). Briefly, Fujitani et al. analyzed the translational polarity in ependymal cells in mice either depleted for p73 during embryogenesis or adulthood (for the definition of polarity see section 1.1.2). By using these two genetically modified mice, they identified that $p 73$ is required for the establishment of translational polarity during embryogenesis but not for the maintenance of translational polarity in adult ependymal cells. Consequently, hydrocephalus development was only observed when depleting p73 during embryogenesis (Fujitani et al., 2017). The effect of p73 on planar cell polarity is attributed to TAp73 isoform, as we demonstrated in collaboration with another group that disrupted planar cell polarity is only observed in TAp73 and p73 KO mice, but not in $D N p 73 \mathrm{KO}$ mice (revised manuscript, FuertesAlvarez,..Wildung et al., 2018). Moreover, we identified that TAp73 regulates planar cell 
polarity by transcriptionally activating the myosin light chain kinase (MLCK), thereby modulating actin dynamics, which is required for proper BB alignment (Supplemental Figure $13 \mathrm{a}$ and $\mathrm{b})$. However, the disrupted motile cilia polarity did neither result in an impaired multiciliogenesis nor in a defective ciliary function in TAp73 KO mice (Figure 22a and b, Figure 25a, Supplemental Figure 9a). As p73 KO mice harbor a reduction of ependymal cilia (Medina-Bolívar et al., 2014), the question arises whether DNp73 compensates for TAp73-driven functions in axoneme formation in the absence of TAp73.

In summary, TAp73 deficiency does not disrupt the multiciliogenesis network in the ventricles of the brain, while it severely impairs multiciliogenesis in all other multiciliated tissues. A similar phenotype has been observed in mice depleted for an upstream factor of TAp73, namely E2f4 as these KO mice possess defective airway multiciliogenesis but no hydrocephalus (Danielian et al., 2007). Only depletion of Gemc1, which interacts with E2F4 or Multicilin, and thus, might represent the most upstream identified regulator of multiciliogenesis, causes ciliary defects in all multiciliated tissues (Terré et al., 2016; Terre et al., 2018).

\subsubsection{In ventricles, a robust network ensures multiciliogenesis in the absence of TAp73}

Studies investigating brain multiciliogenesis including ours suggest that the multiciliogenesis program in the brain is more robust compared to the one in other multiciliated tissues (Danielian et al., 2007; Song et al., 2014). Why is this the case? One major difference between the brain and the airways is their regeneration potential (Kotton and Morrisey, 2014). Ependymal cells are only generated once during embryogenesis (Spassky et al., 2005) and their regenerative capacity after injury is quite low (Kuo et al., 2006; Luo et al., 2008; Luo et al., 2015). In comparison, ciliated cells of the airway epithelium can be rapidly replaced after injury by progenitor cell differentiation and transdifferentiation (Hogan et al., 2014). Thus, we hypothesize that due to the lack of proper repair, ependymal cells build up a more robust multiciliogenesis network to ensure ciliary function while encountering various challenges. Here, we identified two possible players involved in the maintenance of multiciliogenesis in the absence of TAp73 in the brain: E2F4 and miR449, as both are upregulated in TAp73 KO ventricles (Figure 24a and b, Figure 23a and c, Figure $44 b)$. 

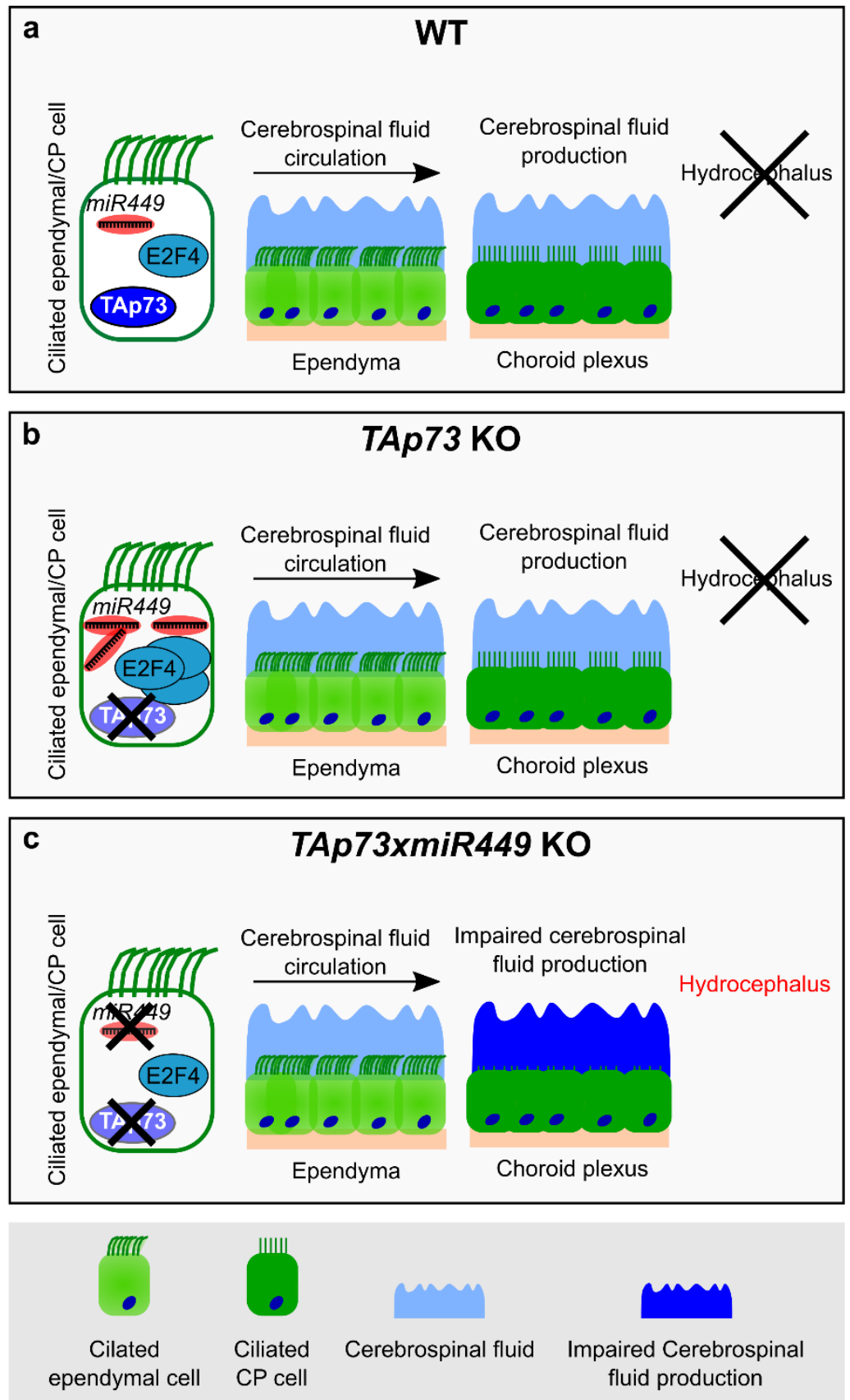

Figure 44: Scheme illustrating the robust network of brain multiciliogenesis.

a) In WT mice, the beating of ependymal cilia is required for the circulation of the cerebrospinal fluid and ciliated CP cells regulate cerebrospinal fluid production. Together, these actions prevent the development of hydrocephalus. b) TAp73 deficiency results in the upregulation of E2F4 and miR449, which jointly maintain the ciliogenesis network. c) Only combined depletion of TAp73 and miR449 reduces CP cilia number and length and results in hydrocephalus. Thus, shorter CP cilia might impair cerebrospinal fluid production and thereby causing hydrocephalus.

When bound to its cofactor Mcidas, E2F4 directly activates the transcription of several prociliogenic factors including TAp73, Foxj1, Rfx2, Rfx3, and Myb (Stubbs et al., 2012; Ma et al., 2014; Spassky and Meunier, 2017). Consistently, depletion of E2f4 results in defective airway multiciliogenesis (Danielian et al., 2007). Interestingly, ectopic expression of an E2f4 form, which bears a generic transactivation domain, along with Mcidas were sufficient to 
induce the formation of motile cilia in MEFs, a cell type that normally generates only primary cilia (Kim et al., 2018). These data illustrate that E2F4 is a potent regulator of multiciliogenesis. Thus, E2F4 upregulation represents one possible explanation for the maintenance of the expression of pro-ciliogenic factors in TAp73 KO brains. Although E2F4 is expressed in cells of the subventricular zone (Ruzhynsky et al., 2007), we ruled out that these cells contribute to the E2F4 upregulation as E2F4 elevation is dependent on TAp73 and TAp73 is only expressed in MCCs of the ventricular zone. Moreover, E2F4 levels seem to be predictive for the ciliary phenotype in TAp73 KO mice as E2F4 is only upregulated in the brains but not in other multiciliated tissues (Supplemental Figure $7 b-e$ ).

In addition to the upregulation of E2F4, we observed increased levels of miR449 in TAp73 $\mathrm{KO}$ brains (Figure 23a and c). miR449 belongs to the miR-34/449 family and since this family is necessary to ensure proper ciliogenesis (discussed in more detail in the sections 5.2.1 and 5.2.3) (Lizé et al., 2010a; Song et al., 2014; Otto et al., 2017), it might also compensate for the loss of TAp73. However, miR449 upregulation upon TAp73 deficiency is a common phenomenon as it is also observed in multiciliated tissues with defective ciliogenesis (Figure 23d). Thus, in FTs, EDs, and airways, miR449 elevation is not sufficient to rescue multiciliogenesis upon TAp73 depletion. The first reason for this could be that the miR449 levels in FTs and tracheae (3-fold) are not as strongly induced as in brains (10-fold) (Figure 23c and d). However, an exception to this are the EDs, which have an even higher induction of miR449 (24-fold) compared to that in the brain, although the variability within the TAp73 KO EDs was considerably high (Figure 23d). Therefore, miR449 upregulation alone is not enough to trigger motile cilia formation. We and others have already shown that miR449 alone is not a potent inducer of ciliogenesis, as depletion of miR449 alone in mice does not affect the generation of motile cilia in diverse tissues under unchallenged conditions (Supplemental Figure 17a and b) (Song et al., 2014; Otto et al., 2017). Only deficiency of all family members (miR-34/449) causes loss of motile cilia (Song et al., 2014; Otto et al., 2017). Therefore, we hypothesize that only the combined upregulation of miR449 along with E2F4 induces the ciliogenesis program in ventricles of TAp73 KO mice (Figure $44 a$ and $b)$.

\subsubsection{Regulatory mechanisms explaining E2F4 and miR449 upregulation upon TAp73 loss}

Nevertheless, the question remains: Why are E2F4 and miR449 upregulated in the brains of TAp73 KO mice? To address this question, we investigated but also propose several different mechanisms, which are described and illustrated below (Figure 45).

To explore the mechanism for the upregulation of E2F4, we first investigated whether Notch signaling is deregulated. Notch signaling represents an interesting target as it promotes CP 
development (Bill et al., 2008; Li et al., 2016), is repressed by miR-34/449 in xenopus (Marcet et al., 2011), and inhibits E2F4/MCIDAS activation (Brooks and Wallingford, 2014). Thus, the observed increase in the levels of miR449 in TAp73 KO animals could induce E2F4 levels via inhibition of Notch signaling (Figure 45).

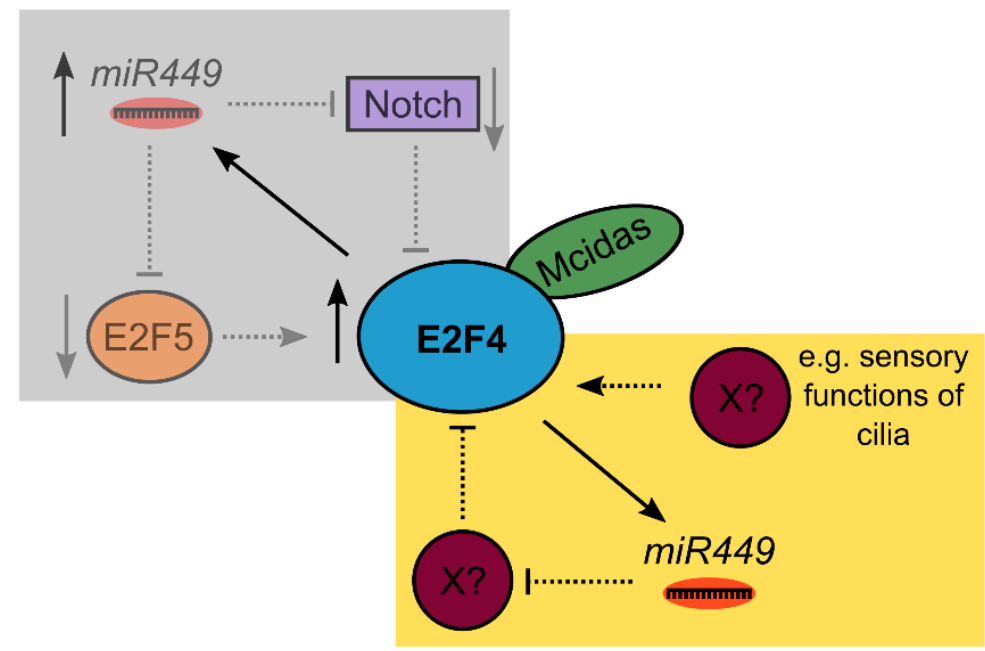

Figure 45: Schematic diagram displaying possible mechanisms involved in the upregulation of E2F4 in TAp73 KO brains.

Grey box illustrates the investigated mechanisms, which do not contribute to E2F4 upregulation. E2F4 upregulation is not due to the downregulation of Notch signaling or E2F5. Yellow box shows proposed mechanisms, which could lead to an elevation of E2F4 but have not yet been studied. Unknown targets of miR449 (X) that inhibit E2F4 or miR449 independent mechanisms (X, e.g. sensory function of cilia) could lead to an upregulation of E2F4.

However, we did not observe an increase of the Notch targets Hes1 and Hes5 in miR449 $\mathrm{KO}$ and TAp73xmiR449 KO mice (Supplemental Figure 11a) and thus, excluded this explanation. In addition, these data suggest that miR449 does not repress the Notch pathway in mice, although it was shown in xenopus. This is in agreement with another dataset showing that the ciliated cell fate in the airway of miR-34/449 $\mathrm{KO}$ mice is not affected, which is usually a consequence of activated Notch signaling (Song et al., 2014). Moreover, another study conducted by Otto et al. supports the species dependency of the interaction between miR449 and Notch as they did not detect altered Notch target levels in the airways of miR-34/449 KO mice (Otto et al., 2017).

Our next hypothesis is based on the link between E2F4, its closely related family member $\mathrm{E} 2 \mathrm{~F} 5$, and miR449. It has been shown that miR449 directly represses E2f5 (Redshaw et al., 2009) and thus, E2F5 might be downregulated upon increased miR449 levels in TAp73 KO mice. As E2F4 and E2F5 possess redundant functions (Gaubatz et al., 2000; Danielian et al., 2016), it is likely that E2F4 transcription is activated to compensate for the loss of E2F5, similar to the compensatory effect between miR34b,c and miR449 (Figure 45) (Bao et al., 2012b; Wu et al., 2014). 
However, our data display unchanged transcript levels of E2f5 in TAp73 KO brains compared to WT brains (data not shown) indicating that E2F4 upregulation might be not due to E2F5 downregulation. As miRNAs often function by inhibiting protein translation without causing mRNA degradation (Bartel, 2018), it would be necessary to confirm this result by protein analysis.

Altogether, our experiments do not provide a reasonable explanation for the upregulation of E2F4. Thus, other unknown targets of miR449 negatively regulating E2F4 might be involved to create a feedback loop between miR449 and E2F4 (Figure 45). Another possible mechanism could involve the sensory function of motile cilia. Motile cilia express several sensory proteins on their plasma membrane, which sense and transduce different kinds of environmental signals (e.g. shear stress, fluid flow, osmotic force) to ensure cellular homeostasis (Jain et al., 2012). For example, the proteins polycystin-1 and 2 act as flow sensors on primary cilia and mutations in these genes result in polycystic kidney disease. Interestingly, polcystin-1 is also abundantly expressed in ciliated ependymal and CP cells, where it is important for ciliary function and signal transduction (Wodarczyk et al., 2009). Thus, it is possible that the upregulation of E2F4 results from signaling cascades initiated by sensory proteins on motile cilia.

The next question that needs to be addressed is the upregulation of miR449 in multiciliated tissues. Here, we explored that E2F4 can directly bind to $\mathrm{Cdc} 20 \mathrm{~b}$ and activate its expression (Figure 24c). miR449 shares the same promotor as Cdc20b (Figure 31a) (Yang et al., 2009; Lizé et al., 2010b) and thus, miR449 upregulation can be partially explained by the increased activity of E2F4 (Figure 46). However, this cannot be the only reason since miR449 upregulation is observed in all other multiciliated tissues depleted for TAp73 where E2F4 levels remain the same (Figure 23d, Supplemental Figure 7b - e). Thus, the increase in miR449 levels seems to be a general phenomenon triggered by TAp73 deficiency. 


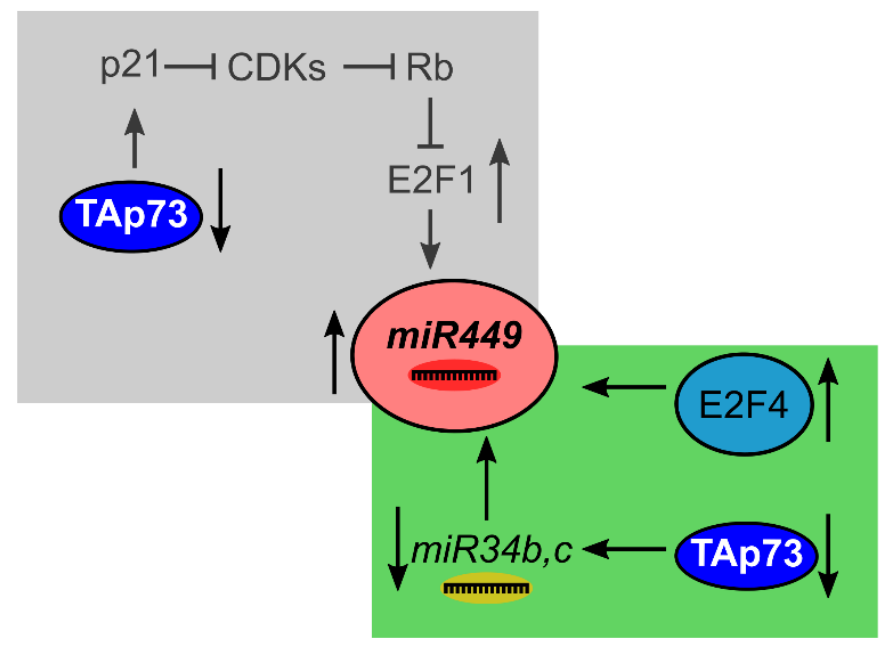

Figure 46: Alterations in the expression of E2F4 and $m i R 34 b, c$ contribute to miR449 upregulation in TAp73 KO brains.

Grey box illustrates a studied signaling cascade, which does not mediate miR449 upregulation in TAp73 KO mice. Theoretically, TAp73 depletion would result in the upregulation miR449 via the p21-Rb-E2F1 axis. Green box displays two mechanisms, which contribute to the increased miR449 levels: 1) E2F4 directly activates the transcription of miR449. 2) Downregulation of the TAp73 target miR34b,c results in upregulation of miR449.

Besides its role in promoting multiciliogenesis, TAp73 inhibits cell cycle progression by activating p21 (Lee and La Thangue, 1999), which at the end results in the inhibition of the cell cycle promoting E2F1 protein (Henley and Dick, 2012). As miR449 has been shown to be E2F1-responsive (Yang et al., 2009; Lizé et al., 2010b), depletion of TAp73 could mediate miR449 upregulation via E2F1 (Figure 46). However, our experiments did not reveal any differences in these cell cycle-associated genes between WT and TAp73 KO mice (Supplemental Figure $8 a$ and $b$ ) and therefore we excluded this possibility.

Previously, we published that TAp73 directly activates miR34b,c expression (Nemajerova et al., 2016). In the present study, we validated this interaction as miR34b,c levels were strongly declined in multiciliated tissues of TAp73 KO mice (Figure 23a and c, Supplemental Figure 7a). Due to the redundant functions of miR34b,c and miR449 (Bao et al., 2012b; Song et al., 2014; Otto et al., 2017), miR449 upregulation might be a consequence of the decreased miR34b,c levels (Figure 46). This compensatory effect has been observed by other groups, which found an upregulation of miR449 in testis, lungs, ovaries, and brains of miR34b,c KO mice (Bao et al., 2012b; Wu et al., 2014).

In summary, we identified two potential mechanisms explaining miR449 upregulation, one via E2F4 and the other via miR34b,c downregulation. However, the mechanism for E2F4 upregulation remains unknown. 


\subsubsection{Combined depletion of TAp73 and the miR449 cluster results in hydrocephalus}

Although, miR449 upregulation alone seems to be insufficient to counterbalance the loss of TAp73 in multiciliated tissues, the importance of its upregulation in this scenario is highlighted by the fact that depletion of miR449 and TAp73 leads to the development of hydrocephalus in mice (Figure 25a, Supplemental Figure 9a). Consistent with the expression of miR449 in the CP (Figure 23b), TAp73xmiR449 KO mice display reduced cilia number and length in the CP epithelium (Figure 25c and d, Supplemental Figure 9b), whereas ciliation of the ependyma is not affected (Supplemental Figure 9c and d). In contrast to ependymal cilia, CP cilia are nonmotile and thus, do not contribute to the circulation of cerebrospinal fluid (Narita et al., 2012). Instead, CP cilia regulate the production of cerebrospinal fluid. Impairment of CP cilia also leads to hydrocephalus (Banizs et al., 2005; Banizs et al., 2007). Therefore, we suggest that combined depletion of TAp73 and miR449 impairs CP ciliogenesis, which results in alterations of the cerebrospinal fluid production and hydrocephalus (Figure 44c).

Interestingly, miR-34/449 KO mice do not phenocopy TAp73xmiR449 KO mice as they do not develop a hydrocephalus (Song et al., 2014), which would have been expected since both mouse models harbor a loss of miR34 and miR449. This illustrates again that the network of multiciliogenesis in the ventricles of the brain is quite robust because only the depletion of two non-related pro-ciliogenic factors (TAp73 and miR-34/449) can disrupt the ciliogenesis program. To prove this, it would be interesting to evaluate whether depletion of E2F4 and TAp73 also results in a similar defective brain multiciliogenesis as observed in TAp73xmiR449 KO mice, since depletion of E2F4 alone does not cause hydrocephalus (Danielian et al., 2007).

Altogether, we identified TAp73 as an important regulator of multiciliogenesis in the male and female reproductive tract. Our study suggests that proper cilia function is required for fertility. Therefore, rescuing cilia generation and motility represent a potential treatment option for PCD patients who suffer from infertility. Furthermore, our investigations of multiciliogenesis in the ventricles emphasize that the transcriptional regulation of multiciliogenesis is tissue-dependent and consist of a complex hierarchy of transcriptional and post-transcriptional regulators, which has not been fully discovered. 


\section{2 miR449 - Post-transcriptional regulation of airway multiciliogenesis}

Besides the transcriptional regulation of multiciliogenesis (e.g. by TAp73), ciliogenesis is additionally fine-tuned at the post-transcriptional level e.g. by miRNAs (Cao et al., 2012; Song et al., 2014; Otto et al., 2017). An important miRNA family involved in the regulation of airway multiciliogenesis is the miR-34/449 family (Song et al., 2014; Otto et al., 2017).

\subsection{1 miR449 KO mice represent a novel model to study the role of motile cilia in COPD}

Here, we demonstrate for the first time that the sole depletion of miR449 influences motile airway cilia homeostasis. In more detail, our data show that miR449 is required for motile cilia maintenance, therefore allowing proper ciliated airway epithelial regeneration upon exposure to various environmental challenges. Consequently, depletion of miR449 in mice combined with exposure to chemicals or infective insults resulted in a loss of motile airway cilia (Figure 33a - c, Figure 34a - d), reduced mucociliary clearance (Figure 40c and d), and increased pulmonary inflammation (Figure $37 \mathrm{a}-\mathrm{f}$ ). The interplay of all these events triggered emphysematous manifestations of COPD in aged miR449 KO mice (Figure 36a c, Figure 37a). Since miR449 seemed to have a protective role in the development of COPD, it would be very interesting to analyze whether miR449 expression is deregulated in COPD patients as well.

Previous studies have claimed that ablation of miR449 alone does not influence multiciliogenesis and only the deletion of the whole family of miR-34/449 results in severe loss of motile airway cilia (Song et al., 2014; Otto et al., 2017). At first glance, this seems to be contrary to our present data. However, a closer look into these studies reveals that these groups evaluated only airway ciliation in young unchallenged miR449 KO mice. This in turn, coincides with our data, which indicates no reduction in cilia number in young miR449 KO mice (Supplemental Figure 16a and b). The novel finding of our study is that miR449 is required for the maintenance of motile airway cilia and ciliated epithelial regeneration and thus, effects in mice with a sole ablation of miR449 are only evident in aged (Figure 35a c) or challenged mice (Figure 33a - c, Figure 34a - d).

In contrast to miR449 KO mice, which are fertile and possess a normal life span, deficiency of all members of the miR-34/449 family results in infertility and early lethality (Song et al., 2014; Otto et al., 2017). Early lethality in miR-34/449 KO mice is due to the loss of airway cilia, which results in defective mucociliary clearance and increased opportunistic infections (Song et al., 2014; Otto et al., 2017). Interestingly, treatment of miR-34/449 KO mice with antibiotics tremendously increased their life span (Otto et al., 2017). Female and male infertility in miR-34/449 $\mathrm{KO}$ mice is caused by the loss of motile cilia in FTs and immotile spermatozoa, respectively (Comazzetto et al., 2014; Song et al., 2014; Wu et al., 2014; Otto 
et al., 2017). Thus, miR-34/449 is required for the formation of motile cilia in the airways and FTs. However, whether miR-34/449 regulate multiciliogenesis in other multiciliated tissues remains unknown.

The comparison between the phenotypes of miR449 $\mathrm{KO}$ and miR-34/449 $\mathrm{KO}$ mice illustrates that the phenotype in miR449 KO mice is much milder compared to the PCD-like phenotype in miR-34/449 KO mice. This indicates that miR34 compensates for the loss of miR449 at least in some respects, although we and others have shown that its levels are not upregulated in the lungs of miR449 KO mice (Figure 31b) (Wu et al., 2014). This is in contrast to the testis of miR449 KO mice, where miR34b,c levels are upregulated and thereby protecting mice from infertility (Bao et al., 2012b; Wu et al., 2014).

Remarkably, it has been shown that the PCD-like phenotype of miR-34/449 KO mice can be rescued by the addition of one allele of miR449 or miR34b,c (Song et al., 2014; Otto et al., 2017). Moreover, we could show that miR449 is more strongly upregulated during mucociliary differentiation (Figure 29b and c). Thus, these data in conjunction with our analysis of miR449 KO mice propose that miR449 is the major contributor that ensures proper (re)generation of the multiciliated airway epithelium. However, it would also be intriguing to investigate the regenerative potential of miR34b,c KO mice, since miR34b,c is also highly expressed in the lungs (Song et al., 2014; Wu et al., 2014; Otto et al., 2017).

Altogether, our data show that miR449 KO mice have a normal life span and only develop ciliary defects upon challenge or with age, which results in manifestations of COPD. As the susceptibility of humans to COPD increases with age and exposure to environmental toxicants (Barnes et al., 2015), the miR449 KO mouse model closely resembles the human situation. Consequently, this genetic mouse model represents a suitable tool to study the role of motile cilia in COPD development.

\subsubsection{Role of motile cilia in chronic respiratory diseases}

Motile airway cilia beat vigorously and thereby help to transport mucus containing trapped particles and pathogens out of the airways. Proper beating of airway cilia is of utmost importance for lung health as any disruption in the ciliary ultrastructure results in mucociliary clearance disorders such as PCD (Tilley et al., 2015). Common mutations found in PCD patients can affect the axonemal dynein arms, which are the motor proteins generating the force required for cilia sliding (Hornef et al., 2006; Zariwala et al., 2006; Loges et al., 2008). The role of motile cilia in inherited ciliary diseases such as PCD is well established. However, the link between motile cilia, mucociliary clearance, and chronic airway diseases is not well described. So far, studies have only shown that COPD patients possess a reduced ciliary beat frequency (Piatti et al., 2005; Yaghi et al., 2012) and cilia length (Hessel et al., 2014). The latter remains controversial as cilia length seems to be unaffected in some 
COPD patient cohorts (Yaghi and Dolovich, 2016). Despite these data, it remains unclear whether cilia dysfunction triggers the onset of COPD or whether it is a consequence of the increased inflammation and airway remodelling occurring during exacerbations.

Here, we provide some new evidence on this topic as we show that deficiency of miR449 disrupts mucociliary clearance (Figure $40 \mathrm{c}$ and $\mathrm{d}$ ) and ciliated epithelial regeneration (Figure $33 a-c$, Figure $34 a-d$, Figure $35 a-c$ ), thereby compromising the innate lung defense, which results in chronic inflammation and COPD development (Figure 36a - c, Figure $37 \mathrm{a}-\mathrm{f}$ ). Since we show that miR449 is specifically expressed in multiciliated tissues (Figure 29a), which is in line with a previous report from Marcet et al. showing miR449 expression exclusively in multiciliated cells (Marcet et al., 2011), our data strongly suggest a link between motile cilia dysfunction and the onset of COPD. Moreover, another study conducted in our lab reinforces the connection between motile cilia dysfunction and COPD. Depletion of the multiciliogenesis regulator TAp73 triggered a COPD-like phenotype with spontaneous emphysema and defective mucociliary clearance (Figure 12a - j) (Nemajerova et al., 2016). In addition, Lam et al. observed that smoking reduces motile airway cilia length via HDAC6-dependent autophagy pathway (Lam et al., 2013). As smoking is one of the main risk factors for COPD (Barnes et al., 2015), motile cilia shortening upon CS exposure might contribute to COPD development.

Moreover, effective mucociliary clearance is important to prevent COPD progression as recurrent pathogen-driven infections drive disease progression and results in a worse prognosis (Barnes et al., 2015). Interestingly, several respiratory pathogens (e.g. Pseudomonas (Wilson et al., 1985; Read et al., 1992), Haemophilus influenzae (Janson et al., 1999; Bailey et al., 2012), viruses (Zhang et al., 2005), and fungi (Piecková and Jesenská, 1996; Bafadhel et al., 2014)), which often colonize COPD lungs (Sethi and Murphy, 2008), have developed ciliostatic factors that impair mucociliary clearance. Consequently, these pathogens are not cleared from the airways and can colonize and damage the lungs.

In summary, as motile cilia are the major contributors to mucociliary clearance, protecting motile airway cilia homeostasis might represent a new treatment option for COPD. The immediate questions that arise are: how is motile cilia homeostasis regulated? How can we target it to protect motile cilia from being disassembled? These two questions will be discussed in terms of miR449-mediated motile cilia maintenance in the following chapters.

\subsubsection{Motile cilia homeostasis is not regulated by well-known miR-34/449 targets in miR449 KO mice}

The miR449 KO mouse represents a good model to identify regulators of motile cilia maintenance as miR449 deficiency reduced the number of motile cilia upon environmental 
challenges (Figure 33a - c, Figure 34a - d). To identify the miR449 targets, which are involved in motile cilia maintenance, we first investigated whether the well-known studied targets of miR-34/449 contributed to the observed phenotype in miR449 KO mice. Previously established targets of miR-34/449 involved in ciliogenesis are: (1) Notch (Marcet et al., 2011), (2) Cp110 (Song et al., 2014), and (3) cell cycle promoting genes (Lizé et al., 2010b; Otto et al., 2017). However, we excluded that the cilia maintenance defect in aged or challenged miR449 KO mice is based on the de-repression of these targets due to the following reasons:

(1) It has been proposed by one study conducted by Marcet et al. that miR449 represses Notch signaling and thereby promotes ciliated cell fate decision (Tsao et al., 2009; Marcet et al., 2011; Carraro and Stripp, 2015). Consequently, the depletion of the miR-34/449 family members would lead to a reduced number of MCCs. Thus, de-repression of Notch in miR449 KO mice could explain the reduction in motile cilia during regeneration processes (Figure 32a - d, Figure 33a - c, Figure 34a - d, Figure 35a - c), which require proliferation of progenitor cells and differentiation into MCCs in order to restore the bronchial epithelium. However, two subsequent studies did not observe a reduction in the number of MCCs in miR-34/449 KO mice. They only noticed that the MCCs express less motile cilia in miR34/449 KO mice (Song et al., 2014; Otto et al., 2017). This indicates that the fate of ciliated cells is unaffected in miR-34/449 KO mice. Moreover, Otto et al. did not detect an upregulation of the Notch signaling pathway in miR-34/449 KO mice (Otto et al., 2017). This agrees with our data from the TAp73 $\mathrm{KO}$ mice, which have an upregulation of miR449 (Figure 23a and c) but no deregulation of the Notch pathway (Supplemental Figure 11a). As Marcet et al. validated the repression of Notch by miR449 only in xenopus (Marcet et al., 2011) and none of the studies performed in mice could confirm the interaction between miR449 and Notch (Song et al., 2014; Otto et al., 2017), we assume that Notch is a speciesspecific target of miR449 and would not be responsible for regulating cilia maintenance in aged or challenged miR449 KO mice.

(2) The second described target of miR-34/449 is Cp110. On the one hand, Cp110 inhibits proper BB maturation and docking (Tsang and Dynlacht, 2013; Song et al., 2014), but on the other hand, Cp110 promotes BB anchorage (Walentek et al., 2016; Yadav et al., 2016). Due to these two opposing roles of $\mathrm{Cp} 110$ in ciliogenesis, its levels must be tightly and timely regulated. In miR-34/449 KO mice, BB are mislocated leading to the loss of motile cilia (Song et al., 2014). However, we did not observe any form of BB mislocalization in ciliated cells of miR449 KO mice (Figure 40a), indicating that Cp110 levels are not affected. This agrees with another report that shows unaltered Cp110 levels in murine airways of miR-34/449 KO mice (Otto et al., 2017). Since the inhibition of Cp110 has been previously described as the main mechanism of miR-34/449-mediated ciliogenesis by rescue 
experiments in xenopus (Song et al., 2014), we concluded that Cp110, like Notch, might be a species-specific target of miR449. Hence, we excluded Cp110 as a miR449 target that could be responsible for the defective ciliated epithelial regeneration in miR449 KO mice.

(3) The third set of miR-34/449 targets that have been described are cell cycle promoting genes (e.g. Cyclin D3, B1, CDC25A). The repression of cell cycle promoting genes by miR$34 / 449$ initiates cell cycle exit, which is a prerequisite for cilia formation (Lizé et al., 2010b; Otto et al., 2017). However, we did not observe reduced numbers of motile cilia in airways of young unchallenged miR449 KO mice (Supplemental Figure 17a and b) and no reduction in the assembly of primary cilia in miR449 KO MEFs (Figure $38 \mathrm{~b}$ and c). Hence, we concluded that the sole depletion of miR449 alone is not sufficient to de-repress the cell cycle machinery in order to inhibit initial motile cilia formation.

However, we cannot exclude the cell cycle regulatory function of miR449 and its impact on cilia formation during the regeneration of the ciliated epithelium. As the regeneration process requires proliferation followed by differentiation into ciliated cells and we observed a reduced formation of motile cilia in aged (Figure 35a -c) and challenged mice (Figure 33ac, Figure $34 \mathrm{a}-\mathrm{d}$ ), it is possible that in this case, miR449 depletion leads to increased proliferation and thereby inhibiting the differentiation into MCCs. To study whether the proliferation rate is increased in challenged miR449 KO mice, an IF staining using a marker for proliferation (e.g. KI-67) could be performed on lung sections from naphthalene-treated WT and miR449 KO mice.

In summary, our experimental data together with the published data suggest that none of the described miR449 targets could explain the defective cilia maintenance phenotype observed in miR449 KO mice. Therefore, the question remains, how does miR449 protect cilia homeostasis and prevent COPD occurrence?

\subsubsection{A new role of $m i R 449$ in protecting motile airway cilia by repressing AURKA-mediated ciliary disassembly}

Having a closer look at the miR449 KO phenotype provides some evidence regarding the mechanism of miR449-mediated cilia homeostasis. Firstly, we identified that depletion of miR449 resulted in loss of motile airway cilia only with age (Figure 35a - c) or upon challenge with respiratory toxicants (Figure 33a - c, Figure 34a - d). Secondly, although both WT and miR449 KO mice showed a decrease in motile cilia one day post naphthalene treatment, we observed a stronger reduction in motile cilia in the miR449 KO mice compared to its WT counterpart (Figure 33a and c). Of note, it has been shown that in addition to club cell exfoliation, ciliated cell squamation followed by motile cilia disassembly occurs one day after naphthalene treatment (van Winkle et al., 1995; van Winkle et al., 1999; Lawson et al., 2002; 
Rawlins et al., 2007). Thirdly, miR449 KO MEFs showed an increased primary cilia disassembly rate with no effect on the assembly rate (Figure $38 \mathrm{~b}$ and $\mathrm{c}$ ). Altogether, these data suggest that miR449 represses ciliary disassembly, thereby protecting cilia maintenance.

So far, the mechanistic information on ciliary disassembly are mainly based on studies that analyzed the homeostasis of primary cilia (Goto et al., 2013; Liang et al., 2016). These studies showed that AURKA-mediated activation of HDAC6 promotes the deacetylation of a-tubulins, which triggers primary cilia disassembly (Figure 39a) (Pan et al., 2004; Pugacheva et al., 2007; Plotnikova et al., 2012; Ran et al., 2015). Although, no studies have directly investigated the molecular mechanisms of motile cilia disassembly, there is evidence, which suggest that motile cilia utilize the same disassembly pathway as primary cilia. Firstly, the structure between primary and motile cilia is quite similar as both are assembled by $\alpha$ - and $\beta$-microtubules using the IFT system (Figure 1b) (Satir and Christensen, 2007b). Secondly, HDAC6 is not only expressed in primary cilia (Pugacheva et al., 2007; Ran et al., 2015), but also localizes to the ciliary axoneme in motile airway cilia (Lam et al., 2013). Last but not least, CS induces the expression of AURKA and HDAC6 and reduces motile cilia length (Lam et al., 2013). Although this study claims that cilia shortening is not likely to be mediated by the deacetylase function of HDAC6, but through the ability of HDAC6 to bind and transport ubiquitinated ciliary proteins to aggresomes, it still indicates that AURKA and HDAC6 are involved in motile cilia homeostasis (Lam et al., 2013).

Our work provides new evidence on this topic and supports the idea that motile cilia disassembly also occurs via the AURKA/HDAC6 axis. Here, we showed that aged and challenged lungs of miR449 KO mice, which harbor less motile cilia, expressed elevated levels of AURKA (Figure 39d - f). In addition to the upregulation of AURKA in vivo, protein as well as transcript levels of AURKA were downregulated upon miR449 overexpression in vitro (Figure 39b and c). Furthermore, the 3'UTR of AURKA possesses predicted binding sites for miR449 (Supplemental Figure 18a). Although we did not perform a reporter-based assay, our data indicate that miR449 could directly repress AURKA and thereby prevents motile cilia disassembly. The connection between miR449 and AURKA is confirmed by another study showing increased AURKA levels in airways and FTs of miR-34/449 KO mice (Song et al., 2014; Otto et al., 2017). Usually, excess levels of AURKA are eliminated by the proteasomal machinery (Lindon et al., 2015). However, as AURKA is still upregulated in miR449 KO mice, it seems that the proteasomal degradation fails to eliminate the excess protein levels. Thus, post-transcriptional regulation by miR449 seems to be another important mechanism to ensure balanced AURKA levels. 
Moreover, it has been shown that elevated levels of AURKA resulted in a dilated appearance of primary cilia (Jacoby et al., 2009; Plotnikova et al., 2015). Remarkably, we also observed similar ultrastructural defects in motile airway cilia as they show ciliary membrane detachments (Figure 40a and b). Thus, we hypothesize that elevated AURKA levels not only destabilize primary cilia but also decrease motile cilia stability in miR449 KO mice. However, as AURKA expression in motile cilia has never been investigated, it is necessary to stain motile airway cilia for AURKA (e.g. IF) in order to confirm our hypothesis. Another follow-up experiment would be to check whether miR449 KO mice possess elevated levels of phosphorylated AURKA (at Thr288), because only if AURKA is phosphorylated it is able to activate HDAC6-mediated disassembly (Pugacheva et al., 2007). The destabilized motile airway cilia in miR449 KO mice could explain the reduced mucociliary clearance, which we identified in mice deficient for miR449 (Figure 40c and d). The connection between defective ciliary ultrastructure and impaired mucociliary clearance is supported by several other studies depicting that structural defects in motile cilia are often associated with mucociliary clearance disorders (Hornef et al., 2006; Zariwala et al., 2006; Tilley et al., 2015).

In summary, our data indicate that miR449 represses AURKA-induced motile cilia disassembly, thereby preventing cilia dysfunction and COPD.

\subsubsection{Does $\operatorname{miR449}$ repress the ciliary disassembly pathway at several nodes?}

miRNAs possess several different targets (Brennecke et al., 2005). To fortify its effect on the ciliary disassembly pathway, it is likely that miR449 represses other targets of the disassembly pathway. Indeed, by using in silico prediction tools (targetScan (Agarwal et al., 2015), PITA (Kertesz et al., 2007)), we predicted two other targets of miR449 within the ciliary disassembly pathway: HDAC6 and KCNH1 (Supplemental Figure 18a).

In comparison to AURKA, HDAC6 has already been associated to mediate motile cilia disassembly upon CS exposure (Lam et al., 2013). However, Lam and colleagues proposed that CS-induced cilia shortening is rather mediated by binding to ubiquitinated ciliary proteins and transporting them along the cytoplasmic microtubule network to aggresomes, where the ciliary proteins are subsequently degraded by autophagy than by the deacetylase function of HDAC6 (Kawaguchi et al., 2003; Lam et al., 2013). Nevertheless, we assume that in the absence of CS, HDAC6 can still promote motile cilia disassembly via its deacetylase activity in miR449 KO mice, since CS inhibits the deacetylase activity of HDAC6. The inhibition of the deacetylase activity of HDAC6 is due an increased acetylation of HDAC6 resulting from decreased levels of its deacetylase Sirtuin-1 upon CS exposure 
(Rajendrasozhan et al., 2008; Liu et al., 2012; Yao et al., 2012; Gal et al., 2013; Lam et al., 2013).

Ciliary disassembly is also induced by increased actin polymerization. Interestingly, HDAC6 promotes actin polymerization by deacetylating cortactin (Ran et al., 2015). Thus, we propose that miR449 also prevents ciliary disassembly by repressing HDAC6 and thereby maintaining a proper actin network. The role of miR449 in actin network formation has been already described. Briefly, it has been shown that miR449 represses Ras-related protein RRas and thereby promotes Filamin A redistribution, which results in the reorganization of the apical actin network essential for proper BB anchoring (Chevalier et al., 2015). Hence, it is likely that miR449 influences actin network formation at different steps to promote cilia generation and maintenance.

The second predicted target of miR449 involved in primary cilia disassembly is the voltagegated potassium channel KCNH1 (Kv10.1) (Sánchez et al., 2016). Overexpression of $K C N H 1$ resulted in an increased disassembly rate of primary cilia via a not yet identified mechanism. One proposed mechanism is that Kv10.1 increases $\mathrm{K}^{+}$permeability, which results in a hyperpolarization of the membrane, which in turn triggers $\mathrm{Ca}^{2+}$ entry into the cell (Sánchez et al., 2016). Ca ${ }^{2+}$ influx is a well-known trigger for the activation of AURKA (Plotnikova et al., 2012). Thus, it can be assumed that Kv10.1 promotes ciliary disassembly by activating the AURKA/HDAC6 pathway. As it has been shown that miR34 represses KCNH1 (Lin et al., 2011a) and miR34 and miR449 share similar targets due to the same seed sequence (Figure 9a), we propose that miR449 represses $K C N H 1$ and thereby interferes with the disassembly pathway at this additional node. Interestingly, SNPs in $K C N H 1$ have been linked to an increased impairment in lung function upon CS exposure (Jong et al., 2017). Although, it represents a first connection between KCNH1 and COPD development, the effect of the SNPs on the function of Kv10.1 remains unknown and these rare SNPs did not reach genome-wide significance (Jong et al., 2017).

Taken together, HDAC6 and $\mathrm{KCNH} 1$ represent two promising candidates that could regulate motile cilia disassembly in the airways of miR449 KO mice. Hence, it would be interesting to further investigate whether these two predicted targets are upregulated in our model system and influence motile cilia disassembly. As in silico prediction tools have a high false positive rate (Pinzón et al., 2017), it is necessary to perform in vitro experiments in order to validate both as true miR449 targets. This can be either done by overexpression of miR449 in cell culture and subsequent staining of HDAC6 and $\mathrm{KCNH} 1$ protein or transcript levels, or by a reporter-based assays. 
In summary, our data suggest that miR449 promotes cilia maintenance by repressing the ciliary disassembly at several nodes, thereby preventing COPD development (Figure 47).

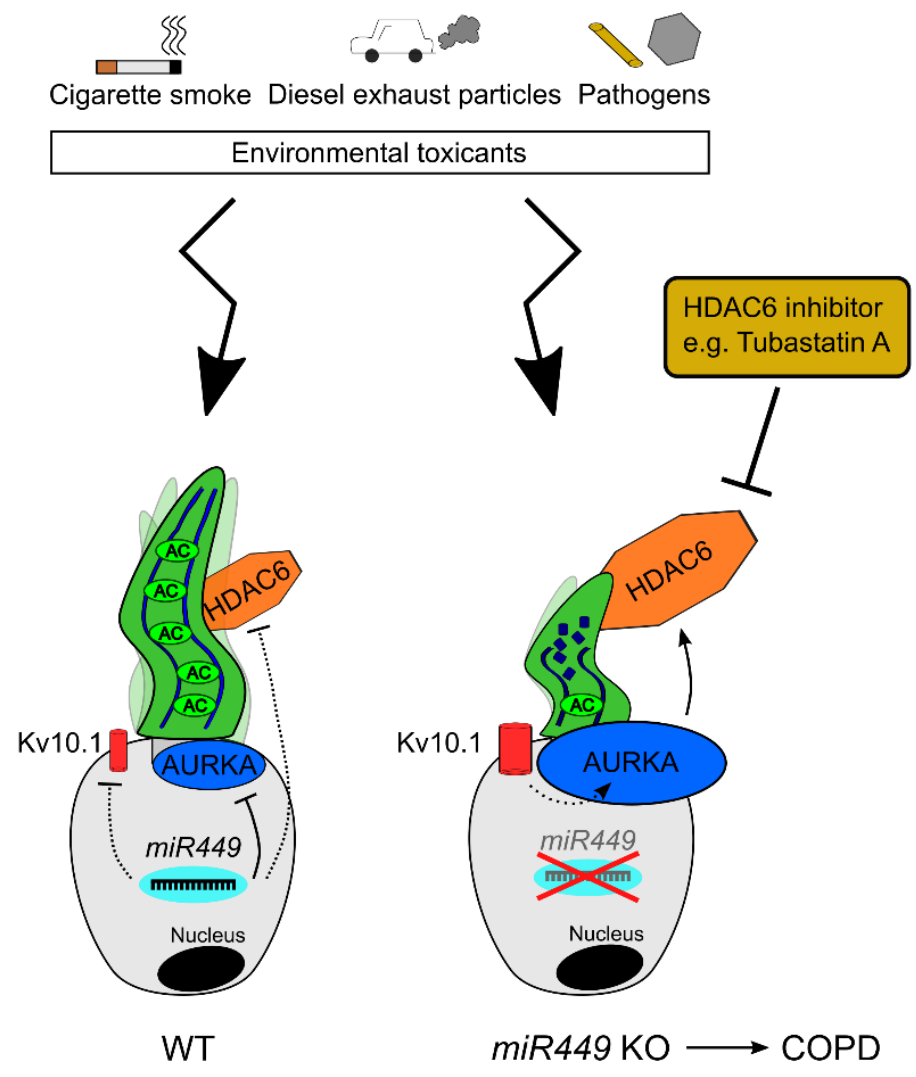

Figure 47: Working model for the role of miR449 in the maintenance of motile airway cilia in response to environmental challenges.

In WT mice, miR449 represses motile cilia disassembly at several nodes, thereby ensuring proper cilia regeneration upon challenge, which is required for healthy lung function. In miR449 KO mice, elevated levels of AURKA promote HDAC6-mediated ciliary disassembly. The resulting impaired ciliated epithelial regeneration in response to environmental challenges triggers manifestations of COPD in miR449 KO mice. Cilia stabilizing agents such as HDAC6 inhibitors might represent a new therapeutic strategy to treat COPD. Continuous lines show validated interaction. Dotted lines indicate predicted connections.

However, to further prove that the reduction of motile cilia in miR449 $\mathrm{KO}$ mice is directly caused by an upregulation of the ciliary disassembly pathway, follow-up experiments are required. One possible experiment would be to treat miR449 KO ALI cultures, which display reduced cilia length (Figure 32c and d, Supplemental Figure 14a), with AURKA and HDAC6 inhibitors to assess potential rescue of ciliary length. If this is the case, the immediate question that arises is whether AURKA or HDAC6 inhibitors can be also used to treat COPD (discussed in section 5.2.6).

Although miR449 is specifically expressed in MCCs of the airway epithelium (Figure 29a) (Lizé et al., 2010a; Marcet et al., 2011), we cannot rule out that other mechanisms independent of MCCs contribute to the COPD phenotype in miR449 KO mice, as we used a systemic KO mouse model. Moreover, it has been shown that miRNAs are expressed in 
exosomes, thus by exosome trafficking miRNAs can be transported from one cell type to another and elicit their function in a paracrine manner (Zhang et al., 2015).

The following mechanisms could contribute to the COPD phenotype in miR449 KO mice (Figure 48).

1) We observed an increased primary cilia disassembly in MEFs from miR449 KO mice compared to WT MEFs (Figure 38b and c) indicating that miR449 deficiency results in a changed fibroblast phenotype. As alterations of the functional properties of lung fibroblasts are proposed to play a role in the pathogenesis of COPD (Togo et al., 2008; Zhang et al., 2012), changes in this cell type might contribute to the COPD phenotype in miR449 KO mice (Figure 48).

2) Moreover, it has been shown that in COPD patients the expression of primary cilia on bronchial epithelia is increased (Perotin et al., 2018). However, it is not known whether primary cilia dysregulation is consequence of airway remodeling or a disease-driving process. Although we rather expect a decrease in primary cilia lining the airways of miR449 KO mice due to the increased primary disassembly rate in miR449 KO MEFs (Figure 38b and c) and not an increase, we cannot rule out that primary cilia dysfunction might be partly responsible for the COPD development in miR449 KO mice (Figure 48).

3) Another possible cell type that could contribute to the COPD phenotype in miR449 $\mathrm{KO}$ mice are pulmonary macrophages. Macrophages secrete pro- and antiinflammatory mediators (e.g. tumor necrosis factor (TNF)- $\alpha$ and interleukin (IL)-10, respectively) and proteases (e.g. MMPs), which degrade elastin fibers leading to the destruction of the alveolar parenchyma (emphysema) (Barnes, 2004). miR449 KO mice developed emphysema with age (Figure 37a), which was accompanied by an increased infiltration of macrophages and elevated MMP levels (Figure 37b - d). We hypothesize that the accumulation of macrophages is a secondary event triggered by an impaired mucociliary clearance resulting in an increased inflammatory response. However, it is also possible that miR449 depletion directly affects macrophages as it has been shown that miR449 is expressed in M2A macrophages (Melton et al., 2016). Moreover, in macrophages, deacetylation of cytoplasmic microtubules by HDAC6 (predicted miR449 target) reduced the secretion of the antiinflammatory cytokine IL-10 (Wang et al., 2014). Thus, it is possible that an altered macrophage polarization results in an impaired resolution of airway inflammation, thereby contributing to the manifestations of COPD in miR449 KO mice (Figure 48). 


\section{DISCUSSION}

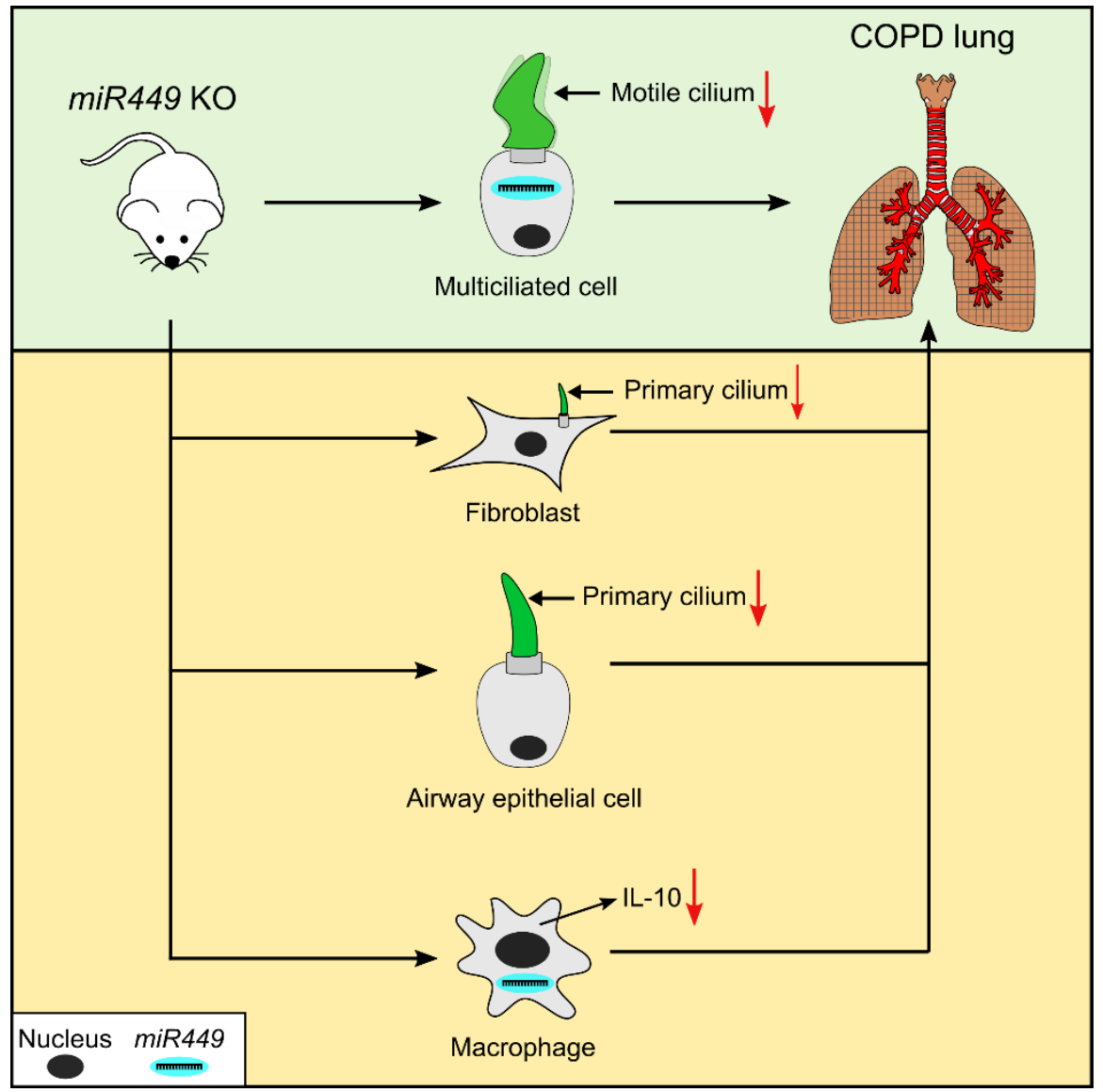

Figure 48: Hypothetical model of mechanisms contributing to the COPD phenotype in miR449 KO mice.

Upper box shows the proposed mechanism that miR449 deficiency impairs motile cilia maintenance and mucociliary clearance resulting in COPD. Lower box depicts alternative mechanisms that could contribute to the COPD phenotype in miR449 KO mice, but these mechanisms have not been investigated in detail in this study. miR449 deficiency might influence the function of other cell types including fibroblasts, airway epithelial cells, and macrophages.

In summary, we cannot exclude that mechanisms other than motile cilia dysfunction including alterations in lung fibroblasts, primary cilia or pulmonary macrophages are involved in the COPD development in miR449 KO mice. To rule out these other mechanisms for the COPD onset in miR449 KO mice, cell-type-specific gene promotors (e.g. FoxJ1 promotor for multiciliogenesiss) in combination with the Cre/LoxP system can be used (Ostrowski et al., 2003; Zhang et al., 2007) to attribute the COPD phenotype to MCCs. 


\subsubsection{Cilia stabilizing agents as a new treatment option for COPD}

As we have shown here that COPD is associated with cilia dysfunction in miR449 KO mice and COPD patients possess a reduced cilia beat frequency (Piatti et al., 2005; Yaghi and Dolovich, 2016) and length (Hessel et al., 2014), we hypothesize that cilia stabilizing agents such as HDAC6 inhibitors might represent a new treatment option for COPD patients (Figure 47). Our hypothesis is supported by a study showing that HDAC6 and AURKA are indeed upregulated in COPD patients (Lam et al., 2013).

AURKA promotes not only ciliary disassembly (Pugacheva et al., 2007; Plotnikova et al., 2012) but also cell cycle progression by ensuring faithful transition through mitosis (Carmena et al., 2009). Thus, its inhibition causes cell cycle defects, which is the reason for using AURKA inhibitors as anti-cancer drugs (Gautschi et al., 2008). However, upon bronchial epithelial injury in COPD patients, proper proliferation of airway epithelial cells is important for the regeneration process in order to restore epithelial function (Beers and Morrisey, 2011). Thus, AURKA inhibitors might not be the drug of choice for the treatment of chronic airway diseases.

HDAC6 inhibitors (e.g. ACY-1215, ACY-241, KA2507) are also under clinical trials for cancer therapy in particular for multiple melanoma (ClinicalTrials.gov Identifier: NCT01323751, ClinicalTrials.gov Identifier: NCT02400242, ClinicalTrials.gov Identifier: NCT03008018 KA2507), but also for non-small cell lung cancer (ClinicalTrials.gov Identifier: NCT02635061). HDAC6 inhibitors are used to treat cancer as HDAC6 mediates degradation of ubiquitinated misfolded proteins via the aggresome pathway (Kawaguchi et al., 2003), which is one of the many survival pathways for cancer cells (Rodriguez-Gonzalez et al., 2008). As this function of HDAC6 has also been shown to trigger CS-induced motile airway cilia shortening, HDAC6 selective inhibitors might represent a new therapeutic option for COPD. Moreover, HDAC6 KO mice do not display any severe defects (Zhang et al., 2008), which indicates that HDAC6 inhibition by pharmacological compounds would not elicit strong side effects in humans. HDAC6 inhibition would not only have a positive effect on cilia maintenance but would also promote the resolution of airway inflammation by inhibiting cytoplasmic microtubule deacetylation and thereby inducing IL-10 secretion (Wang et al., 2014). Thus, HDAC6 inhibitors represent a potent drug candidate. One newly identified and highly selective HDAC6 inhibitor is tubastatin A (Butler et al., 2010), which is not yet listed in clinical trials.

To date, there are no available treatments to cure COPD and to restore the functionality of the airway epithelium. Thus, motile cilia stabilizing agents might represent a new therapeutic strategy to treat COPD. 


\subsection{Conclusion}

In conclusion, our work has highlighted that TAp73 and miR449 are crucial for proper formation and maintenance of motile cilia in different multiciliated organs.

We established that TAp73 is an important central regulator of multiciliogenesis in many multiciliated tissues, including airways (Nemajerova et al., 2016), EDs, and FTs. It activates the transcription of several pro-ciliogenic genes, thereby facilitating motile cilia formation. Moreover, our data suggest that proper transcriptional regulation of multiciliogenesis by TAp73 is important to ensure female and male fertility. In contrast, TAp73 is dispensable for brain multiciliogenesis and only depletion of both TAp73 and miR449 impairs brain cilia homeostasis, leading to hydrocephalus. This indicates that the transcriptional and posttranscriptional network of multiciliogenesis is more robust in the brain. Consequently, multiciliogenesis is differently regulated in multiciliated tissues. However, more research is required to fully understand the precise interactions of this complex network.

Besides the importance of proper motile cilia formation for human health, we demonstrated that the maintenance of motile airway cilia in response to environmental challenges is equally important for assuring lung health. We identified miR449 as a key player in this process as its depletion disrupted ciliated epithelial regeneration accompanied by defective mucociliary clearance resulting in COPD manifestations in aged miR449 KO mice. Our data suggest that miR449 prevents COPD onset by repressing the ciliary disassembly pathway and by protecting proper motile cilia regeneration. Thus, targeting cilia maintenance might represent a new strategy to restore ciliary function in COPD. As not only motile cilia but also primary cilia defects are involved in the pathogenesis of diseases, looking into other diseases associated with defective primary cilia such as renal diseases (e.g. polycystic kidney disease (Cao et al., 2009)) might also be of interest for the application of cilia stabilizing agents. Altogether, tight regulation of motile cilia formation and maintenance by TAp73 and miR449 is essential for the functionality of several processes in diverse multiciliated organs. Therefore, it would be interesting to study whether mutations of TAp73 and miR449 are found in patients with cilia dysfunction. 


\section{REFERENCES}

Agarwal, V., Bell, G.W., Nam, J.-W., and Bartel, D.P. (2015) Predicting effective microRNA target sites in mammalian mRNAs. Elife 4.

Amirav, I., Wallmeier, J., Loges, N.T., Menchen, T., Pennekamp, P., Mussaffi, H., et al. (2016) Systematic Analysis of CCNO Variants in a Defined Population: Implications for Clinical Phenotype and Differential Diagnosis. Human Mutation 37 (4): 396-405.

Arbi, M., Pefani, D.-E., Kyrousi, C., Lalioti, M.-E., Kalogeropoulou, A., Papanastasiou, A.D., et al. (2016) GemC1 controls multiciliogenesis in the airway epithelium. EMBO Rep. 17 (3): 400-413.

Avasthi, P., and Marshall, W.F. (2012) Stages of ciliogenesis and regulation of ciliary length. Differentiation 83 (2): S30-42.

Avidor-Reiss, T., and Gopalakrishnan, J. (2013) Cell Cycle Regulation of the Centrosome and Cilium. Drug Discov Today Dis Mech 10 (3-4): e119-e124.

Baek, D., Villen, J., Shin, C., Camargo, F.D., Gygi, S.P., and Bartel, D.P. (2008) The impact of microRNAs on protein output. Nature 455 (7209): 64-71.

Bafadhel, M., McKenna, S., Agbetile, J., Fairs, A., Desai, D., Mistry, V., et al. (2014) Aspergillus fumigatus during stable state and exacerbations of COPD. Eur Respir $\mathrm{J} \mathbf{4 3}$ (1): 64-71.

Bailey, K.L., LeVan, T.D., Yanov, D.A., Pavlik, J.A., DeVasure, J.M., Sisson, J.H., and Wyatt, T.A. (2012) Non-typeable Haemophilus influenzae decreases cilia beating via protein kinase $\mathrm{C} \varepsilon$. Respir Res 13: 49.

Baldwin, R.M., Jewell, W.T., Fanucchi, M.V., Plopper, C.G., and Buckpitt, A.R. (2004) Comparison of pulmonary/nasal CYP2F expression levels in rodents and rhesus macaque. J Pharmacol Exp Ther 309 (1): 127-136.

Banizs, B., Komlosi, P., Bevensee, M.O., Schwiebert, E.M., Bell, P.D., and Yoder, B.K. (2007) Altered $\mathrm{pH}(\mathrm{i})$ regulation and $\mathrm{Na}(+) / \mathrm{HCO} 3(-)$ transporter activity in choroid plexus of cilia-defective Tg737(orpk) mutant mouse. Am J Physiol, Cell Physiol 292 (4): C1409-16.

Banizs, B., Pike, M.M., Millican, C.L., Ferguson, W.B., Komlosi, P., Sheetz, J., et al. (2005) Dysfunctional cilia lead to altered ependyma and choroid plexus function, and result in the formation of hydrocephalus. Development 132 (23): 5329-5339.

Bao, J., Li, D., Wang, L., Wu, J., Hu, Y., Wang, Z., et al. (2012a) MicroRNA-449 and microRNA-34b/c function redundantly in murine testes by targeting E2F transcription factor-retinoblastoma protein (E2F-pRb) pathway. J Biol Chem 287 (26): 21686-21698.

Bao, J., Li, D., Wang, L., Wu, J., Hu, Y., Wang, Z., et al. (2012b) MicroRNA-449 and MicroRNA-34b/c Function Redundantly in Murine Testes by Targeting E2F Transcription Factor-Retinoblastoma Protein (E2F-pRb) Pathway. J Biol Chem 287 (26): 21686-21698.

Barnes, P.J. (2004) Mediators of chronic obstructive pulmonary disease. Pharmacol Rev 56 (4): 515-548.

Barnes, P.J., Burney, P.G.J., Silverman, E.K., Celli, B.R., Vestbo, J., Wedzicha, J.A., and Wouters, E.F.M. (2015) Chronic obstructive pulmonary disease. Nat Rev Dis Primers 1: 15076.

Bartel, D.P. (2018) Metazoan MicroRNAs. Cell 173 (1): 20-51.

Beers, M.F., and Morrisey, E.E. (2011) The three R's of lung health and disease: repair, remodeling, and regeneration. J Clin Invest 121 (6): 2065-2073.

Bill, B.R., Balciunas, D., McCarra, J.A., Young, E.D., Xiong, T., Spahn, A.M., et al. (2008) Development and Notch Signaling Requirements of the Zebrafish Choroid Plexus. PLoS One 3 (9): e3114. 
Blatt, E.N., Yan, X.H., Wuerffel, M.K., Hamilos, D.L., and Brody, S.L. (1999) Forkhead transcription factor HFH-4 expression is temporally related to ciliogenesis. Am J Respir Cell Mol Biol 21 (2): 168-176.

Boon, M., Wallmeier, J., Ma, L., Loges, N.T., Jaspers, M., Olbrich, H., et al. (2014) MCIDAS mutations result in a mucociliary clearance disorder with reduced generation of multiple motile cilia. Nat Commun 5: 4418.

Boucher, R.C. (2007) Evidence for airway surface dehydration as the initiating event in CF airway disease. J Intern Med 261 (1): 5-16.

Brennecke, J., Stark, A., Russell, R.B., and Cohen, S.M. (2005) Principles of microRNAtarget recognition. PLoS Biology 3 (3): e85.

Brody, S.L., Yan, X.H., Wuerffel, M.K., Song, S.K., and Shapiro, S.D. (2000) Ciliogenesis and left-right axis defects in forkhead factor HFH-4-null mice. Am J Respir Cell Mol Biol 23 (1): 45-51.

Brooks, E.R., and Wallingford, J.B. (2014) Multiciliated cells. Curr Biol 24 (19): R973-82.

Buckpitt, A., Boland, B., Isbell, M., Morin, D., Shultz, M., Baldwin, R., et al. (2002) Naphthalene-induced respiratory tract toxicity: metabolic mechanisms of toxicity. Drug Metab Rev 34 (4): 791-820.

Buckpitt, A., Chang, A.M., Weir, A., van Winkle, L., Duan, X., Philpot, R., and Plopper, C. (1995) Relationship of cytochrome P450 activity to Clara cell cytotoxicity. IV. Metabolism of naphthalene and naphthalene oxide in microdissected airways from mice, rats, and hamsters. Mol Pharmacol 47 (1): 74-81.

Bustamante-Marin, X.M., and Ostrowski, L.E. (2017) Cilia and Mucociliary Clearance. Cold Spring Harb Perspect Biol 9 (4).

Butler, K.V., Kalin, J., Brochier, C., Vistoli, G., Langley, B., and Kozikowski, A.P. (2010) Rational design and simple chemistry yield a superior, neuroprotective HDAC6 inhibitor, tubastatin A. J Am Chem Soc 132 (31): 10842-10846.

Cao, J., Shen, Y., Zhu, L., Xu, Y., Zhou, Y., Wu, Z., et al. (2012) miR-129-3p controls cilia assembly by regulating CP110 and actin dynamics. Nat Cell Biol 14 (7): 697-706.

Cao, Y., Semanchik, N., Lee, S.H., Somlo, S., Barbano, P.E., Coifman, R., and Sun, Z. (2009) Chemical modifier screen identifies HDAC inhibitors as suppressors of PKD models. Proc Natl Acad Sci U S A 106 (51): 21819-21824.

Capece, V., Garcia Vizcaino, J.C., Vidal, R., Rahman, R.-U., Pena Centeno, T., Shomroni, O., et al. (2015) Oasis: online analysis of small RNA deep sequencing data. Bioinformatics 31 (13): 2205-2207.

Carmena, M., Ruchaud, S., and Earnshaw, W.C. (2009) Making the Auroras glow: regulation of Aurora A and B kinase function by interacting proteins. Curr Opin Cell Biol 21 (6): 796-805.

Carraro, G., and Stripp, B.R. (2015) A new notch for lung stem cells. Cell Stem Cell 16 (2): 107-109.

Caspary, T., Larkins, C.E., and Anderson, K.V. (2007) The Graded Response to Sonic Hedgehog Depends on Cilia Architecture. Developmental Cell 12 (5): 767-778.

Chen, J., Knowles, H.J., Hebert, J.L., and Hackett, B.P. (1998) Mutation of the mouse hepatocyte nuclear factor/forkhead homologue 4 gene results in an absence of cilia and random left-right asymmetry. J Clin Invest 102 (6): 1077-1082.

Chevalier, B., Adamiok, A., Mercey, O., Revinski, D.R., Zaragosi, L.-E., Pasini, A., et al. (2015) miR-34/449 control apical actin network formation during multiciliogenesis through small GTPase pathways. Nat Commun 6: 8386.

Chhin, B., Negre, D., Merrot, O., Pham, J., Tourneur, Y., Ressnikoff, D., et al. (2009) Ciliary beating recovery in deficient human airway epithelial cells after lentivirus ex vivo gene therapy. PLoS Genet 5 (3): e1000422.

Choksi, S.P., Lauter, G., Swoboda, P., and Roy, S. (2014) Switching on cilia: transcriptional networks regulating ciliogenesis. Development 141 (7): 1427-1441. 
Chung, M.-I., Kwon, T., Tu, F., Brooks, E.R., Gupta, R., Meyer, M., et al. (2014) Coordinated genomic control of ciliogenesis and cell movement by RFX2. Elife 3: e01439.

Chung, M.-I., Peyrot, S.M., LeBoeuf, S., Park, T.J., McGary, K.L., Marcotte, E.M., and Wallingford, J.B. (2012) RFX2 is broadly required for ciliogenesis during vertebrate development. Dev Biol 363 (1): 155-165.

Comazzetto, S., Di Giacomo, M., Rasmussen, K.D., Much, C., Azzi, C., Perlas, E., et al. (2014) Oligoasthenoteratozoospermia and infertility in mice deficient for miR-34b/c and miR-449 loci. PLoS Genet 10 (10): e1004597.

Cooke, H.J., and Saunders, P.T.K. (2002) Mouse models of male infertility. Nat Rev Genet 3 (10): 790-801.

Crocker, J. C., et al. (1996) Methods of Digital Video Microscopy for Colloidal Studies. J Colloid Interface Sci 179 (217): 298-310.

Dacheux, J.-L., and Dacheux, F. (2013) New insights into epididymal function in relation to sperm maturation. Reproduction 147 (2): R27-R42.

Danielian, P.S., Bender Kim, C.F., Caron, A.M., Vasile, E., Bronson, R.T., and Lees, J.A. (2007) E2f4 is required for normal development of the airway epithelium. Dev Biol 305 (2): 564-576.

Danielian, P.S., Hess, R.A., and Lees, J.A. (2016) E2f4 and E2f5 are essential for the development of the male reproductive system. Cell Cycle 15 (2): 250-260.

Dawe, H.R., Farr, H., and Gull, K. (2007) Centriole/basal body morphogenesis and migration during ciliogenesis in animal cells. J Cell Sci 120 (Pt 1): 7-15.

Diez-Roux, G., Banfi, S., Sultan, M., Geffers, L., Anand, S., Rozado, D., et al. (2011) A High-Resolution Anatomical Atlas of the Transcriptome in the Mouse Embryo. PLoS Biology 9 (1): e1000582.

Dobin, A., Davis, C.A., Schlesinger, F., Drenkow, J., Zaleski, C., Jha, S., et al. (2013) STAR: ultrafast universal RNA-seq aligner. Bioinformatics 29 (1): 15-21.

Doench, J.G., and Sharp, P.A. (2004) Specificity of microRNA target selection in translational repression. Genes Dev 18 (5): 504-511.

Ezzati, M., Djahanbakhch, O., Arian, S., and Carr, B.R. (2014) Tubal transport of gametes and embryos: a review of physiology and pathophysiology. J Assist Reprod Genet 31 (10): 1337-1347.

Faubel, R., Westendorf, C., Bodenschatz, E., and Eichele, G. (2016) Cilia-based flow network in the brain ventricles. Science 353 (6295): 176-178.

Feng, Z., Zhang, C., Kang, H.-J., Sun, Y., Wang, H., Naqvi, A., et al. (2011) Regulation of female reproduction by p53 and its family members. FASEB J. 25 (7): 2245-2255.

Fliegauf, M., Benzing, T., and Omran, H. (2007) When cilia go bad: cilia defects and ciliopathies. Nat Rev Mol Cell Biol 8 (11): 880-893.

Fox, J.G., Barthold, S., and Davisson, M. (2006) The Mouse in Biomedical Research: Normative Biology, Husbandry, and Models. s.I.: Elsevier professional.

Friedländer, M.R., Mackowiak, S.D., Li, N., Chen, W., and Rajewsky, N. (2012) miRDeep2 accurately identifies known and hundreds of novel microRNA genes in seven animal clades. Nucleic Acids Res. 40 (1): 37-52.

Fujitani, M., Sato, R., and Yamashita, T. (2017) Loss of p73 in ependymal cells during the perinatal period leads to aqueductal stenosis. Scientific Reports 7 (1): 12007.

Funk, M.C., Bera, A.N., Menchen, T., Kuales, G., Thriene, K., Lienkamp, S.S., et al. (2015) Cyclin $\mathrm{O}$ (Ccno) functions during deuterosome-mediated centriole amplification of multiciliated cells. EMBO J. 34 (8): 1078-1089.

Gal, J., Chen, J., Barnett, K.R., Yang, L., Brumley, E., and Zhu, H. (2013) HDAC6 regulates mutant SOD1 aggregation through two SMIR motifs and tubulin acetylation. $J$ Biol Chem 288 (21): 15035-15045. 
Gallinas, S.C., and Lizé, M. (2013) MIR449A (microRNA 449a). Atlas of Genetics and Cytogenetics in Oncology and Haematology (3).

Garcia, G., and Reiter, J.F. (2016) A primer on the mouse basal body. Cilia 5: 17.

Gaubatz, S., Lindeman, G.J., Ishida, S., Jakoi, L., Nevins, J.R., Livingston, D.M., and Rempel, R.E. (2000) E2F4 and E2F5 play an essential role in pocket protein-mediated G1 control. Molecular Cell 6 (3): 729-735.

Gautschi, O., Heighway, J., Mack, P.C., Purnell, P.R., Lara, P.N., and Gandara, D.R. (2008) Aurora kinases as anticancer drug targets. Clinical Cancer Research 14 (6): 1639-1648.

Giangreco, A., Reynolds, S.D., and Stripp, B.R. (2002) Terminal Bronchioles Harbor a Unique Airway Stem Cell Population That Localizes to the Bronchoalveolar Duct Junction. Am J Pathol 161 (1): 173-182.

Gomperts, B.N., Gong-Cooper, X., and Hackett, B.P. (2004) Foxj1 regulates basal body anchoring to the cytoskeleton of ciliated pulmonary epithelial cells. J Cell Sci 117 (Pt 8): 1329-1337.

Gomperts, B.N., Kim, L.J., Flaherty, S.A., and Hackett, B.P. (2007) IL-13 regulates cilia loss and foxj1 expression in human airway epithelium. Am J Respir Cell Mol Biol 37 (3): 339-346.

Goto, H., Inoko, A., and Inagaki, M. (2013) Cell cycle progression by the repression of primary cilia formation in proliferating cells. Cell Mol Life Sci 70 (20): 3893-3905.

Goutaki, M., Meier, A.B., Halbeisen, F.S., Lucas, J.S., Dell, S.D., Maurer, E., et al. (2016) Clinical manifestations in primary ciliary dyskinesia: systematic review and metaanalysis. Eur Respir J 48 (4): 1081-1095.

Grove, K.C. de, Provoost, S., Brusselle, G.G., Joos, G.F., and Maes, T. (2018) Insights in particulate matter-induced allergic airway inflammation: Focus on the epithelium. Clin Exp Allergy 48 (7): 773-786.

Gu, X., Karp, P.H., Brody, S.L., Pierce, R.A., Welsh, M.J., Holtzman, M.J., and BenShahar, Y. (2014) Chemosensory functions for pulmonary neuroendocrine cells. Am J Respir Cell Mol Biol 50 (3): 637-646.

Guglielmino, M.R., Santonocito, M., Vento, M., Ragusa, M., Barbagallo, D., Borzì, P., et al. (2011) TAp73 is downregulated in oocytes from women of advanced reproductive age. Cell Cycle 10 (19): 3253-3256.

Guichard, C.?c., Harricane, M.-C.?c., Lafitte, J.-J., Godard, P., Zaegel, M., Tack, V., et al. (2001) Axonemal Dynein Intermediate-Chain Gene (DNAl1) Mutations Result in Situs Inversus and Primary Ciliary Dyskinesia (Kartagener Syndrome). Am J Hum Genet 68 (4): 1030-1035.

Hackett, B.P., Brody, S.L., Liang, M., Zeitz, I.D., Bruns, L.A., and Gitlin, J.D. (1995) Primary structure of hepatocyte nuclear factor/forkhead homologue 4 and characterization of gene expression in the developing respiratory and reproductive epithelium. Proc Natl Acad Sci U S A 92 (10): 4249-4253.

Hamer, G., Gademan, I.S., Kal, H.B., and Rooij, D.G. de (2001) Role for c-Abl and p73 in the radiation response of male germ cells. Oncogene 20 (32): 4298-4304.

Han, S., and Mallampalli, R.K. (2015) The Role of Surfactant in Lung Disease and Host Defense against Pulmonary Infections. Ann Am Thorac Soc 12 (5): 765-774.

Harrod, K.S., Jaramillo, R.J., Berger, J.A., Gigliotti, A.P., Seilkop, S.K., and Reed, M.D. (2005) Inhaled diesel engine emissions reduce bacterial clearance and exacerbate lung disease to Pseudomonas aeruginosa infection in vivo. Toxicol Sci 83 (1): 155165.

Henley, S.A., and Dick, F.A. (2012) The retinoblastoma family of proteins and their regulatory functions in the mammalian cell division cycle. Cell Div 7 (1): 10.

Hermeking, H. (2010) The miR-34 family in cancer and apoptosis. Cell Death Differ 17 (2): 193-199. 
Hess, R.A. (2002) The Efferent Ductules: Structure and Functions. In The Epididymis: From Molecules to Clinical Practice. Robaire, B., and Hinton, B.T. (ed.). Boston, MA: Springer US, pp. 49-80.

Hess, R.A. (2015) Small tubules, surprising discoveries: from efferent ductules in the turkey to the discovery that estrogen receptor alpha is essential for fertility in the male. Anim Reprod 12 (1): 7-23.

Hessel, J., Heldrich, J., Fuller, J., Staudt, M.R., Radisch, S., Hollmann, C., et al. (2014) Intraflagellar transport gene expression associated with short cilia in smoking and COPD. PLoS One 9 (1): e85453.

Hiemstra, P.S., and Bourdin, A. (2014) Club cells, CC10 and self-control at the epithelial surface. Eur Respir J 44 (4): 831-832.

Hogan, B.L.M., Barkauskas, C.E., Chapman, H.A., Epstein, J.A., Jain, R., Hsia, C.C.W., et al. (2014) Repair and regeneration of the respiratory system: complexity, plasticity, and mechanisms of lung stem cell function. Cell Stem Cell 15 (2): 123-138.

Holembowski, L., Kramer, D., Riedel, D., Sordella, R., Nemajerova, A., Dobbelstein, M., and Moll, U.M. (2014) TAp73 is essential for germ cell adhesion and maturation in testis. J Cell Biol 204 (7): 1173-1190.

Horani, A., and Ferkol, T.W. (2016) Primary ciliary dyskinesia and associated sensory ciliopathies. Expert Rev Respir Med 10 (5): 569-576.

Hornef, N., Olbrich, H., Horvath, J., Zariwala, M.A., Fliegauf, M., Loges, N.T., et al. (2006) DNAH5 mutations are a common cause of primary ciliary dyskinesia with outer dynein arm defects. Am J Respir Crit Care Med 174 (2): 120-126.

Houghton, A.M. (2015) Matrix metalloproteinases in destructive lung disease. Matrix Biol 44-46: 167-174.

Hu, W., Zheng, T., and Wang, J. (2011) Regulation of Fertility by the p53 Family Members. Genes \& Cancer 2 (4): 420-430.

Huang, K., Diener, D.R., and Rosenbaum, J.L. (2009a) The ubiquitin conjugation system is involved in the disassembly of cilia and flagella. J Cell Biol 186 (4): 601-613.

Huang, X., Ketova, T., Fleming, J.T., Wang, H., Dey, S.K., Litingtung, Y., and Chiang, C. (2009b) Sonic hedgehog signaling regulates a novel epithelial progenitor domain of the hindbrain choroid plexus. Development 136 (15): 2535-2543.

Ibanez-Tallon, I. (2004) Dysfunction of axonemal dynein heavy chain Mdnah5 inhibits ependymal flow and reveals a novel mechanism for hydrocephalus formation. Hum Mol Genet 13 (18): 2133-2141.

Ibañez-Tallon, I., Gorokhova, S., and Heintz, N. (2002) Loss of function of axonemal dynein Mdnah5 causes primary ciliary dyskinesia and hydrocephalus. Hum Mol Genet 11 (6): 715-721.

Ilio, K.Y., and Hess, R.A. (1994) Structure and function of the ductuli efferentes: a review. Microsc. Res. Tech. 29 (6): 432-467.

Inaba, H., Goto, H., Kasahara, K., Kumamoto, K., Yonemura, S., Inoko, A., et al. (2016) Ndel1 suppresses ciliogenesis in proliferating cells by regulating the trichoplein-Aurora A pathway. J Cell Biol 212 (4): 409-423.

Inoko, A., Matsuyama, M., Goto, H., Ohmuro-Matsuyama, Y., Hayashi, Y., Enomoto, M., et al. (2012) Trichoplein and Aurora A block aberrant primary cilia assembly in proliferating cells. J Cell Biol 197 (3): 391-405.

Inoue, S., Tomasini, R., Rufini, A., Elia, A.J., Agostini, M., Amelio, I., et al. (2014) TAp73 is required for spermatogenesis and the maintenance of male fertility. Proc Natl Acad Sci U S A 111 (5): 1843-1848.

Ishikawa, H., and Marshall, W.F. (2011) Ciliogenesis: building the cell's antenna. Nat Rev Mol Cell Biol 12 (4): 222-234. 
Jacoby, M., Cox, J.J., Gayral, S., Hampshire, D.J., Ayub, M., Blockmans, M., et al. (2009) INPP5E mutations cause primary cilium signaling defects, ciliary instability and ciliopathies in human and mouse. Nat Genet 41 (9): 1027-1031.

Jain, R., Javidan-Nejad, C., Alexander-Brett, J., Horani, A., Cabellon, M.C., Walter, M.J., and Brody, S.L. (2012) Sensory functions of motile cilia and implication for bronchiectasis. Front Biosci (Schol Ed) 4: 1088-1098.

Janson, H., Carl n, B., Cervin, A., Forsgren, A., Magnusdottir, A.B., Lindberg, S., and Runer, T. (1999) Effects on the ciliated epithelium of protein D-producing and nonproducing nontypeable Haemophilus influenzae in nasopharyngeal tissue cultures. J. Infect. Dis. 180 (3): 737-746.

Jiménez, A.J., Domínguez-Pinos, M.-D., Guerra, M.M., Fernández-Llebrez, P., and PérezFígares, J.-M. (2014) Structure and function of the ependymal barrier and diseases associated with ependyma disruption. Tissue Barriers 2: e28426.

Johnson, A.P., and Inzana, T.J. (1986) Loss of ciliary activity in organ cultures of rat trachea treated with lipo-oligosaccharide from Haemophilus influenzae. J Med Microbiol 22 (3): 265-268.

Jong, K. de, Vonk, J.M., Imboden, M., Lahousse, L., Hofman, A., Brusselle, G.G., et al. (2017) Genes and pathways underlying susceptibility to impaired lung function in the context of environmental tobacco smoke exposure. Respir Res 18 (1): 142.

Junek, S., Chen, T.-W., Alevra, M., and Schild, D. (2009) Activity correlation imaging: visualizing function and structure of neuronal populations. Biophys J 96 (9): $3801-$ 3809.

Kawaguchi, Y., Kovacs, J.J., McLaurin, A., Vance, J.M., Ito, A., and Yao, T.P. (2003) The deacetylase HDAC6 regulates aggresome formation and cell viability in response to misfolded protein stress. Cell 115 (6): 727-738.

Kertesz, M., lovino, N., Unnerstall, U., Gaul, U., and Segal, E. (2007) The role of site accessibility in microRNA target recognition. Nat Genet 39 (10): 1278-1284.

Kim, S., Ma, L., Shokhirev, M.N., Quigley, I., and Kintner, C. (2018) Multicilin and activated E2f4 induce multiciliated cell differentiation in primary fibroblasts. Scientific Reports 8 (1): 12369.

King, P.T., and Sharma, R. (2015) The Lung Immune Response to Nontypeable Haemophilus influenzae (Lung Immunity to NTHi). J Immunol Res 2015: 706376.

Kinzel, D., Boldt, K., Davis, E.E., Burtscher, I., Trümbach, D., Diplas, B., et al. (2010) Pitchfork regulates primary cilia disassembly and left-right asymmetry. Developmental Cell 19 (1): 66-77.

Knust, J., Ochs, M., Gundersen, H.J.G., and Nyengaard, J.R. (2009) Stereological estimates of alveolar number and size and capillary length and surface area in mice lungs. Anat Rec (Hoboken) 292 (1): 113-122.

Kobayashi, T., and Dynlacht, B.D. (2011) Regulating the transition from centriole to basal body. J Cell Biol 193 (3): 435-444.

Koeppel, M., van Heeringen, S.J., Kramer, D., Smeenk, L., Janssen-Megens, E., Hartmann, M., et al. (2011) Crosstalk between c-Jun and TAp73alpha/beta contributes to the apoptosis-survival balance. Nucleic Acids Res. 39 (14): 6069-6085.

Kotton, D.N., and Morrisey, E.E. (2014) Lung regeneration: mechanisms, applications and emerging stem cell populations. Nat Med 20 (8): 822-832.

Krasteva, G., Canning, B.J., Hartmann, P., Veres, T.Z., Papadakis, T., Mühlfeld, C., et al. (2011) Cholinergic chemosensory cells in the trachea regulate breathing. Proc Natl Acad Sci U S A 108 (23): 9478-9483.

Kunimoto, K., Yamazaki, Y., Nishida, T., Shinohara, K., Ishikawa, H., Hasegawa, T., et al. (2012) Coordinated ciliary beating requires Odf2-mediated polarization of basal bodies via basal feet. Cell 148 (1-2): 189-200. 
Kuo, C.T., Mirzadeh, Z., Soriano-Navarro, M., Rasin, M., Wang, D., Shen, J., et al. (2006) Postnatal deletion of Numb/Numblike reveals repair and remodeling capacity in the subventricular neurogenic niche. Cell 127 (6): 1253-1264.

Kyrousi, C., Arbi, M., Pilz, G.-A., Pefani, D.-E., Lalioti, M.-E., Ninkovic, J., et al. (2015) Mcidas and GemC1 are key regulators for the generation of multiciliated ependymal cells in the adult neurogenic niche. Development 142 (21): 3661-3674.

Kyrousi, C., Lalioti, M.-E., Skavatsou, E., Lygerou, Z., and Taraviras, S. (2016) Mcidas and GemC1/Lynkeas specify embryonic radial glial cells. Neurogenesis 3 (1): e1172747.

Lam, H.C., Cloonan, S.M., Bhashyam, A.R., Haspel, J.A., Singh, A., Sathirapongsasuti, J.F., et al. (2013) Histone deacetylase 6-mediated selective autophagy regulates COPD-associated cilia dysfunction. J Clin Invest 123 (12): 5212-5230.

Lapperre, T.S., Postma, D.S., Gosman, M.M.E., Snoeck-Stroband, J.B., Hacken, N.H.T. ten, Hiemstra, P.S., et al. (2006) Relation between duration of smoking cessation and bronchial inflammation in COPD. Thorax 61 (2): 115-121.

Lawson, G.W., van Winkle, L.S., Toskala, E., Senior, R.M., Parks, W.C., and Plopper, C.G. (2002) Mouse Strain Modulates the Role of the Ciliated Cell in Acute Tracheobronchial Airway Injury-Distal Airways. Am J Pathol 160 (1): 315-327.

Lee, C.W., and La Thangue, N.B. (1999) Promoter specificity and stability control of the p53-related protein p73. Oncogene 18 (29): 4171-4181.

Lee, L. (2013) Riding the wave of ependymal cilia: Genetic susceptibility to hydrocephalus in primary ciliary dyskinesia: Ependymal Cilia and PCD. Journal of Neuroscience Research 91 (9): 1117-1132.

Li, L., Grausam, K.B., Wang, J., Lun, M.P., Ohli, J., Lidov, H.G.W., et al. (2016) Sonic Hedgehog promotes proliferation of Notch-dependent monociliated choroid plexus tumour cells. Nat Cell Biol 18 (4): 418-430.

Li, L., Wei, Y., van Winkle, L., Zhang, Q.-Y., Zhou, X., Hu, J., et al. (2011) Generation and characterization of a Cyp2f2-null mouse and studies on the role of CYP2F2 in naphthalene-induced toxicity in the lung and nasal olfactory mucosa. $J$ Pharmacol Exp Ther 339 (1): 62-71.

Liang, Y., Meng, D., Zhu, B., and Pan, J. (2016) Mechanism of ciliary disassembly. Cell Mol Life Sci 73 (9): 1787-1802.

Liao, Y., Smyth, G.K., and Shi, W. (2014) featureCounts: an efficient general purpose program for assigning sequence reads to genomic features. Bioinformatics 30 (7): 923930.

Lin, H., Li, Z., Chen, C., Luo, X., Xiao, J., Dong, D., et al. (2011a) Correction: Transcriptional and Post-Transcriptional Mechanisms for Oncogenic Overexpression of Ether À Go-Go K + Channel. PLOS ONE 6 (11).

Lin, H., Li, Z., Chen, C., Luo, X., Xiao, J., Dong, D., et al. (2011b) Transcriptional and Post-Transcriptional Mechanisms for Oncogenic Overexpression of Ether À Go-Go K+ Channel. PLOS ONE 6 (5).

Lindeman, G.J., Dagnino, L., Gaubatz, S., Xu, Y., Bronson, R.T., Warren, H.B., and Livingston, D.M. (1998) A specific, nonproliferative role for E2F-5 in choroid plexus function revealed by gene targeting. Genes Dev. 12 (8): 1092-1098.

Lindon, C., Grant, R., and Min, M. (2015) Ubiquitin-Mediated Degradation of Aurora Kinases. Front Oncol 5: 307.

Liu, Y., Peng, L., Seto, E., Huang, S., and Quu, Y. (2012) Modulation of histone deacetylase 6 (HDAC6) nuclear import and tubulin deacetylase activity through acetylation. J Biol Chem 287 (34): 29168-29174.

Lizé, M., Herr, C., Klimke, A., Bals, R., and Dobbelstein, M. (2010a) MicroRNA-449a levels increase by several orders of magnitude during mucociliary differentiation of airway epithelia. Cell Cycle 9 (22): 4579-4583. 
Lizé, M., Klimke, A., and Dobbelstein, M. (2011) MicroRNA-449 in cell fate determination. Cell Cycle 10 (17): 2874-2882.

Lizé, M., Pilarski, S., and Dobbelstein, M. (2010b) E2F1-inducible microRNA 449a/b suppresses cell proliferation and promotes apoptosis. Cell Death Differ 17 (3): 452458.

Loges, N.T., Olbrich, H., Fenske, L., Mussaffi, H., Horvath, J., Fliegauf, M., et al. (2008) DNAI2 mutations cause primary ciliary dyskinesia with defects in the outer dynein arm. Am J Hum Genet 83 (5): 547-558.

Love, M.I., Huber, W., and Anders, S. (2014) Moderated estimation of fold change and dispersion for RNA-seq data with DESeq2. Genome Biol. 15 (12): 550.

Luo, J., Shook, B.A., Daniels, S.B., and Conover, J.C. (2008) Subventricular zonemediated ependyma repair in the adult mammalian brain. J Neurosci 28 (14): 3804 3813.

Luo, Y., Coskun, V., Liang, A., Yu, J., Cheng, L., Ge, W., et al. (2015) Single-cell transcriptome analyses reveal signals to activate dormant neural stem cells. Cell 161 (5): 1175-1186.

Lyons, R.A., Saridogan, E., and Djahanbakhch, O. (2006) The reproductive significance of human Fallopian tube cilia. Hum. Reprod. Update 12 (4): 363-372.

Ma, L., Quigley, I., Omran, H., and Kintner, C. (2014) Multicilin drives centriole biogenesis via E2f proteins. Genes Dev. 28 (13): 1461-1471.

Marcet, B., Chevalier, B., Luxardi, G., Coraux, C., Zaragosi, L.-E., Cibois, M., et al. (2011) Control of vertebrate multiciliogenesis by miR-449 through direct repression of the Delta/Notch pathway. Nat Cell Biol 13 (6): 693-699.

Marshall, C.B., Mays, D.J., Beeler, J.S., Rosenbluth, J.M., Boyd, K.L., Santos Guasch, G.L., et al. (2016) p73 Is Required for Multiciliogenesis and Regulates the Foxj1Associated Gene Network. Cell Rep 14 (10): 2289-2300.

Martin, M. (2011) Cutadapt removes adapter sequences from high-throughput sequencing reads. EMBnet.journal 17 (1): 10.

Medina-Bolívar, C., González-Arnay, E., Talos, F., González-Gómez, M., Moll, U.M., and Meyer, G. (2014) Cortical hypoplasia and ventriculomegaly of p73-deficient mice: Developmental and adult analysis: p73 in developing and adult cortex. Journal of Comparative Neurology 522 (11): 2663-2679.

Melino, G., Laurenzi, V. de, and Vousden, K.H. (2002) p73: Friend or foe in tumorigenesis. Nat Rev Cancer 2 (8): 605-615.

Melton, D.W., Lei, X., Gelfond, J.A.L., and Shireman, P.K. (2016) Dynamic macrophage polarization-specific miRNA patterns reveal increased soluble VEGF receptor 1 by miR-125a-5p inhibition. Physiol Genomics 48 (5): 345-360.

Mendive, F., Laurent, P., van Schoore, G., Skarnes, W., Pochet, R., and Vassart, G. (2006) Defective postnatal development of the male reproductive tract in LGR4 knockout mice. Dev Biol 290 (2): 421-434.

Mercey, O., Popa, A., Cavard, A., Paquet, A., Chevalier, B., Pons, N., et al. (2017) Characterizing isomiR variants within the microRNA-34/449 family. FEBS Lett 591 (5): 693-705.

Milla, C.E. (2016) The evolving spectrum of ciliopathies and respiratory disease. Curr Opin Pediatr 28 (3): 339-347.

Montoro, D.T., Haber, A.L., Biton, M., Vinarsky, V., Lin, B., Birket, S.E., et al. (2018) A revised airway epithelial hierarchy includes CFTR-expressing ionocytes. Nature 560 (7718): 319-324.

Murphy, D.B., Seemann, S., Wiese, S., Kirschner, R., Grzeschik, K.H., and Thies, U. (1997) The human hepatocyte nuclear factor 3/fork head gene FKHL13: genomic structure and pattern of expression. Genomics 40 (3): 462-469. 
Narita, K., Kozuka-Hata, H., Nonami, Y., Ao-Kondo, H., Suzuki, T., Nakamura, H., et al. (2012) Proteomic analysis of multiple primary cilia reveals a novel mode of ciliary development in mammals. Biol Open 1 (8): 815-825.

Narita, K., and Takeda, S. (2015) Cilia in the choroid plexus: their roles in hydrocephalus and beyond. Front Cell Neurosci 9: 39.

Nemajerova, A., Kramer, D., Siller, S.S., Herr, C., Shomroni, O., Pena, T., et al. (2016) TAp73 is a central transcriptional regulator of airway multiciliogenesis. Genes Dev. 30 (11): 1300-1312.

Núnez-Ollé, M., Jung, C., Terré, B., Balsiger, N.A., Plata, C., Roset, R., et al. (2017) Constitutive Cyclin $\mathrm{O}$ deficiency results in penetrant hydrocephalus, impaired growth and infertility. Oncotarget 8 (59): 99261-99273.

Ohata, S., Nakatani, J., Herranz-Pérez, V., Cheng, J., Belinson, H., Inubushi, T., et al. (2014) Loss of Dishevelleds disrupts planar polarity in ependymal motile cilia and results in hydrocephalus. Neuron 83 (3): 558-571.

Ostrowski, L.E., Hutchins, J.R., Zakel, K., and O'Neal, W.K. (2003) Targeting expression of a transgene to the airway surface epithelium using a ciliated cell-specific promoter. Mol Ther 8 (4): 637-645.

Otto, T., Candido, S.V., Pilarz, M.S., Sicinska, E., Bronson, R.T., Bowden, M., et al. (2017) Cell cycle-targeting microRNAs promote differentiation by enforcing cell-cycle exit. Proc Natl Acad Sci U S A 114 (40): 10660-10665.

Pan, J., Wang, Q., and Snell, W.J. (2004) An aurora kinase is essential for flagellar disassembly in Chlamydomonas. Developmental Cell 6 (3): 445-451.

Park, K.-S., Wells, J.M., Zorn, A.M., Wert, S.E., Laubach, V.E., Fernandez, L.G., and Whitsett, J.A. (2006) Transdifferentiation of ciliated cells during repair of the respiratory epithelium. Am J Respir Cell Mol Biol 34 (2): 151-157.

Perotin, J.-M., Coraux, C., Lagonotte, E., Birembaut, P., Delepine, G., Polette, M., et al. (2018) Alteration of primary cilia in COPD. Eur Respir J 52 (1).

Piatti, G., Ambrosetti, U., Santus, P., and Allegra, L. (2005) Effects of salmeterol on cilia and mucus in COPD and pneumonia patients. Pharmacol Res 51 (2): 165-168.

Piecková, E., and Jesenská, Z. (1996) Ciliostatic effect of fungi on the respiratory tract ciliary movement of one-day-old chickens in vitro. Folia Microbiol (Praha) 41 (6): 517520.

Pinzón, N., Li, B., Martinez, L., Sergeeva, A., Presumey, J., Apparailly, F., and Seitz, H. (2017) microRNA target prediction programs predict many false positives. Genome Research 27 (2): 234-245.

Plasschaert, L.W., Žilionis, R., Choo-Wing, R., Savova, V., Knehr, J., Roma, G., et al. (2018) A single-cell atlas of the airway epithelium reveals the CFTR-rich pulmonary ionocyte. Nature 560 (7718): 377-381.

Plopper, C.G., Suverkropp, C., Morin, D., Nishio, S., and Buckpitt, A. (1992) Relationship of cytochrome P-450 activity to Clara cell cytotoxicity. I. Histopathologic comparison of the respiratory tract of mice, rats and hamsters after parenteral administration of naphthalene. J Pharmacol Exp Ther 261 (1): 353-363.

Plotnikova, O.V., Nikonova, A.S., Loskutov, Y.V., Kozyulina, P.Y., Pugacheva, E.N., and Golemis, E.A. (2012) Calmodulin activation of Aurora-A kinase (AURKA) is required during ciliary disassembly and in mitosis. Mol Biol Cell 23 (14): 2658-2670.

Plotnikova, O.V., Seo, S., Cottle, D.L., Conduit, S., Hakim, S., Dyson, J.M., et al. (2015) INPP5E interacts with AURKA, linking phosphoinositide signaling to primary cilium stability. J Cell Sci 128 (2): 364-372.

Pozniak, C.D. (2000) An Anti-Apoptotic Role for the p53 Family Member, p73, During Developmental Neuron Death. Science 289 (5477): 304-306. 
Pozniak, C.D., Barnabé-Heider, F., Rymar, V.V., Lee, A.F., Sadikot, A.F., and Miller, F.D. (2002) p73 is required for survival and maintenance of CNS neurons. $J$ Neurosci 22 (22): 9800-9809.

Provoost, S., Grove, K.C. de, Fraser, G.L., Lannoy, V.J., Tournoy, K.G., Brusselle, G.G., et al. (2016) Pro- and Anti-Inflammatory Role of ChemR23 Signaling in PollutantInduced Inflammatory Lung Responses. J Immunol 196 (4): 1882-1890.

Pugacheva, E.N., Jablonski, S.A., Hartman, T.R., Henske, E.P., and Golemis, E.A. (2007) HEF1-dependent Aurora A activation induces disassembly of the primary cilium. Cell 129 (7): 1351-1363.

Rackley, C.R., and Stripp, B.R. (2012) Building and maintaining the epithelium of the lung. J Clin Invest 122 (8): 2724-2730.

Rajendrasozhan, S., Yang, S.-R., Kinnula, V.L., and Rahman, I. (2008) SIRT1, an antiinflammatory and antiaging protein, is decreased in lungs of patients with chronic obstructive pulmonary disease. Am J Respir Crit Care Med 177 (8): 861-870.

Ran, J., Yang, Y., Li, D., Liu, M., and Zhou, J. (2015) Deacetylation of a-tubulin and cortactin is required for HDAC6 to trigger ciliary disassembly. Scientific Reports 5: 12917 EP -.

Rawlins, E.L., Ostrowski, L.E., Randell, S.H., and Hogan, B.L.M. (2007) Lung development and repair: contribution of the ciliated lineage. Proc Natl Acad Sci U S A 104 (2): 410-417.

Read, R.C., Roberts, P., Munro, N., Rutman, A., Hastie, A., Shryock, T., et al. (1992) Effect of Pseudomonas aeruginosa rhamnolipids on mucociliary transport and ciliary beating. J Appl Physiol (1985) 72 (6): 2271-2277.

Redshaw, N., Wheeler, G., Hajihosseini, M.K., and Dalmay, T. (2009) microRNA-449 is a putative regulator of choroid plexus development and function. Brain Research 1250: 20-26.

Reid, L., Meyrick, B., Antony, V.B., Chang, L.-Y., Crapo, J.D., and Reynolds, H.Y. (2005) The mysterious pulmonary brush cell: a cell in search of a function. Am J Respir Crit Care Med 172 (1): 136-139.

Reynolds, S.D., Giangreco, A., Power, J.H.T., and Stripp, B.R. (2000) Neuroepithelial Bodies of Pulmonary Airways Serve as a Reservoir of Progenitor Cells Capable of Epithelial Regeneration. Am J Pathol 156 (1): 269-278.

Rodriguez-Gonzalez, A., Lin, T., Ikeda, A.K., Simms-Waldrip, T., Fu, C., and Sakamoto, K.M. (2008) Role of the aggresome pathway in cancer: targeting histone deacetylase 6-dependent protein degradation. Cancer Res 68 (8): 2557-2560.

Rogers, D.F. (2005) Mucociliary dysfunction in COPD: effect of current pharmacotherapeutic options. Pulm Pharmacol Ther 18 (1): 1-8.

Ross, A.J., Dailey, L.A., Brighton, L.E., and Devlin, R.B. (2007) Transcriptional profiling of mucociliary differentiation in human airway epithelial cells. Am J Respir Cell Mol Biol 37 (2): 169-185.

Ruz, R., Gregory, M., Smith, C.E., Cyr, D.G., Lubahn, D.B., Hess, R.A., and Hermo, L. (2006) Expression of aquaporins in the efferent ductules, sperm counts, and sperm motility in estrogen receptor-alpha deficient mice fed lab chow versus casein. $\mathrm{Mol}$ Reprod Dev 73 (2): 226-237.

Ruzhynsky, V.A., McClellan, K.A., Vanderluit, J.L., Jeong, Y., Furimsky, M., Park, D.S., et al. (2007) Cell cycle regulator E2F4 is essential for the development of the ventral telencephalon. J Neurosci 27 (22): 5926-5935.

Sánchez, A., Urrego, D., and Pardo, L.A. (2016) Cyclic expression of the voltage-gated potassium channel KV10.1 promotes disassembly of the primary cilium. EMBO Rep. 17 (5): 708-723.

Sánchez, I., and Dynlacht, B.D. (2016) Cilium assembly and disassembly. Nat Cell Biol 18 (7): 711-717. 
Satir, P., and Christensen, S.T. (2007a) Overview of structure and function of mammalian cilia. Annu Rev Physio/ 69: 377-400.

Satir, P., and Christensen, S.T. (2007b) Overview of Structure and Function of Mammalian Cilia. Annu Rev Physiol 69 (1): 377-400.

Satir, P., Heuser, T., and Sale, W.S. (2014) A Structural Basis for How Motile Cilia Beat. BioScience 64 (12): 1073-1083.

Schilders, K.A.A., Eenjes, E., van Riet, S., Poot, A.A., Stamatialis, D., Truckenmüller, R., et al. (2016) Regeneration of the lung: Lung stem cells and the development of lung mimicking devices. Respir Res 17: 44.

Schindelin, J., Arganda-Carreras, I., Frise, E., Kaynig, V., Longair, M., Pietzsch, T., et al. (2012) Fiji: an open-source platform for biological-image analysis. Nat. Methods 9 (7): 676-682.

Sethi, S., and Murphy, T.F. (2008) Infection in the pathogenesis and course of chronic obstructive pulmonary disease. N Engl J Med 359 (22): 2355-2365.

Shah, V.S., Meyerholz, D.K., Tang, X.X., Reznikov, L., Abou Alaiwa, M., Ernst, S.E., et al. (2016) Airway acidification initiates host defense abnormalities in cystic fibrosis mice. Science 351 (6272): 503-507.

Shu, J., Li, D., Ouyang, H., Huang, J., Long, Z., Liang, Z., et al. (2017) Comparison and evaluation of two different methods to establish the cigarette smoke exposure mouse model of COPD. Scientific Reports 7 (1): 15454.

Song, R., Walentek, P., Sponer, N., Klimke, A., Lee, J.S., Dixon, G., et al. (2014) miR$34 / 449$ miRNAs are required for motile ciliogenesis by repressing cp110. Nature 510 (7503): 115-120.

Spassky, N., Merkle, F.T., Flames, N., Tramontin, A.D., García-Verdugo, J.M., and Alvarez-Buylla, A. (2005) Adult ependymal cells are postmitotic and are derived from radial glial cells during embryogenesis. J. Neurosci. 25 (1): 10-18.

Spassky, N., and Meunier, A. (2017) The development and functions of multiciliated epithelia. Nat Rev Mol Cell Biol 18 (7): 423-436.

Staudt, M.R., Buro-Auriemma, L.J., Walters, M.S., Salit, J., Vincent, T., Shaykhiev, R., et al. (2014) Airway Basal stem/progenitor cells have diminished capacity to regenerate airway epithelium in chronic obstructive pulmonary disease. Am J Respir Crit Care Med 190 (8): 955-958.

Stubbs, J.L., Vladar, E.K., Axelrod, J.D., and Kintner, C. (2012) Multicilin promotes centriole assembly and ciliogenesis during multiciliate cell differentiation. Nat Cell Biol 14 (2): 140-147.

Studer, D., Michel, M., and Müller, M. (1989) High pressure freezing comes of age. Scanning Microsc Supp/ 3: 253-68; discussion 268-9.

Subramanian, A., Tamayo, P., Mootha, V.K., Mukherjee, S., Ebert, B.L., Gillette, M.A., et al. (2005) Gene set enrichment analysis: a knowledge-based approach for interpreting genome-wide expression profiles. Proc Natl Acad Sci U S A 102 (43): 15545-15550.

Sui, P., Wiesner, D.L., Xu, J., Zhang, Y., Lee, J., van Dyken, S., et al. (2018) Pulmonary neuroendocrine cells amplify allergic asthma responses. Science 360 (6393).

Talos, F., Abraham, A., Vaseva, A.V., Holembowski, L., Tsirka, S.E., Scheel, A., et al. (2010) p73 is an essential regulator of neural stem cell maintenance in embryonal and adult CNS neurogenesis. Cell Death Differ 17 (12): 1816-1829.

Tam, A., Wadsworth, S., Dorscheid, D., Man, S F Paul, and Sin, D.D. (2011) The airway epithelium: more than just a structural barrier. Ther Adv Respir Dis 5 (4): 255-273.

Tan, F.E., Vladar, E.K., Ma, L., Fuentealba, L.C., Hoh, R., Espinoza, F.H., et al. (2013) Myb promotes centriole amplification and later steps of the multiciliogenesis program. Development 140 (20): 4277-4286. 
Tasena, H., Faiz, A., Timens, W., Noordhoek, J., Hylkema, M.N., Gosens, R., et al. (2018) microRNA-mRNA regulatory networks underlying chronic mucus hypersecretion in COPD. Eur Respir J.

Terre, B., Lewis, M., Gil-Gomez, G., and Stracker, T.H. (2018) GEMC1 and CCNO are required for efferent duct development and male fertility.

Terré, B., Piergiovanni, G., Segura-Bayona, S., Gil-Gómez, G., Youssef, S.A., Attolini, C.S.-O., et al. (2016) GEMC1 is a critical regulator of multiciliated cell differentiation. EMBO J. 35 (9): 942-960.

Tilley, A.E., Walters, M.S., Shaykhiev, R., and Crystal, R.G. (2015) Cilia dysfunction in lung disease. Annu Rev Physiol 77: 379-406.

Tissir, F., Ravni, A., Achouri, Y., Riethmacher, D., Meyer, G., and Goffinet, A.M. (2009) DeltaNp73 regulates neuronal survival in vivo. Proc Natl Acad Sci U S A 106 (39): 16871-16876.

Togo, S., Holz, O., Liu, X., Sugiura, H., Kamio, K., Wang, X., et al. (2008) Lung Fibroblast Repair Functions in Patients with Chronic Obstructive Pulmonary Disease Are Altered by Multiple Mechanisms. Am J Respir Crit Care Med 178 (3): 248-260.

Tomasini, R., Tsuchihara, K., Tsuda, C., Lau, S.K., Wilhelm, M., Ruffini, A., et al. (2009) TAp73 regulates the spindle assembly checkpoint by modulating BubR1 activity. Proc Natl Acad Sci U S A 106 (3): 797-802.

Tomasini, R., Tsuchihara, K., Wilhelm, M., Fujitani, M., Rufini, A., Cheung, C.C., et al. (2008) TAp73 knockout shows genomic instability with infertility and tumor suppressor functions. Genes Dev. 22 (19): 2677-2691.

Trimarchi, J.M., and Lees, J.A. (2002) Sibling rivalry in the E2F family. Nat Rev Mol Cell Biol 3 (1): 11-20.

Tsang, W.Y., and Dynlacht, B.D. (2013) CP110 and its network of partners coordinately regulate cilia assembly. Cilia 2 (1): 9.

Tsao, P.-N., Vasconcelos, M., Izvolsky, K.I., Qian, J., Lu, J., and Cardoso, W.V. (2009) Notch signaling controls the balance of ciliated and secretory cell fates in developing airways. Development 136 (13): 2297-2307.

Tulsiani, D.R.P., and Abou-Haila, A. (2012) Biological Processes that Prepare Mammalian Spermatozoa to Interact with an Egg and Fertilize It. Scientifica (Cairo) 2012: 607427.

Turner, J., Roger, J., Fitau, J., Combe, D., Giddings, J., van Heeke, G., and Jones, C.E. (2011) Goblet cells are derived from a FOXJ1-expressing progenitor in a human airway epithelium. Am J Respir Cell Mol Biol 44 (3): 276-284.

Tyner, J.W., Kim, E.Y., Ide, K., Pelletier, M.R., Roswit, W.T., Morton, J.D., et al. (2006) Blocking airway mucous cell metaplasia by inhibiting EGFR antiapoptosis and IL-13 transdifferentiation signals. J Clin Invest 116 (2): 309-321.

van Winkle, L.S., Buckpitt, A.R., Nishio, S.J., Isaac, J.M., and Plopper, C.G. (1995) Cellular response in naphthalene-induced Clara cell injury and bronchiolar epithelial repair in mice. Am. J. Physiol. 269 (6 Pt 1): L800-18.

van Winkle, L.S., Gunderson, A.D., Shimizu, J.A., Baker, G.L., and Brown, C.D. (2002) Gender differences in naphthalene metabolism and naphthalene-induced acute lung injury. Am J Physiol Lung Cell Mol Physiol 282 (5): L1122-34.

van Winkle, L.S., Johnson, Z.A., Nishio, S.J., Brown, C.D., and Plopper, C.G. (1999) Early events in naphthalene-induced acute Clara cell toxicity: comparison of membrane permeability and ultrastructure. Am J Respir Cell Mol Biol 21 (1): 44-53.

Vanaken, G.J., Bassinet, L., Boon, M., Mani, R., Honoré, I., Papon, J.-F., et al. (2017) Infertility in an adult cohort with primary ciliary dyskinesia: phenotype-gene association. Eur Respir J 50 (5): 1700314.

Vikhreva, P., Melino, G., and Amelio, I. (2018) p73 Alternative Splicing: Exploring a Biological Role for the C-Terminal Isoforms. J Mol Biol 430 (13): 1829-1838. 
Volckaert, T., Dill, E., Campbell, A., Tiozzo, C., Majka, S., Bellusci, S., and Langhe, S.P. de (2011) Parabronchial smooth muscle constitutes an airway epithelial stem cell niche in the mouse lung after injury. J Clin Invest 121 (11): 4409-4419.

Walentek, P., Quigley, I.K., Sun, D.I., Sajjan, U.K., Kintner, C., and Harland, R.M. (2016) Ciliary transcription factors and miRNAs precisely regulate $\mathrm{Cp} 110$ levels required for ciliary adhesions and ciliogenesis. Elife 5.

Wallingford, J.B. (2010) Planar cell polarity signaling, cilia and polarized ciliary beating. Curr Opin Cell Biol 22 (5): 597-604.

Wallmeier, J., Al-Mutairi, D.A., Chen, C.-T., Loges, N.T., Pennekamp, P., Menchen, T., et al. (2014) Mutations in CCNO result in congenital mucociliary clearance disorder with reduced generation of multiple motile cilia. Nat Genet 46 (6): 646-651.

Wang, B., Rao, Y.-H., Inoue, M., Hao, R., Lai, C.-H., Chen, D., et al. (2014) Microtubule acetylation amplifies p38 kinase signalling and anti-inflammatory IL-10 production. Nat Commun 5: 3479.

West, J.A., Pakehham, G., Morin, D., Fleschner, C.A., Buckpitt, A.R., and Plopper, C.G. (2001) Inhaled naphthalene causes dose dependent Clara cell cytotoxicity in mice but not in rats. Toxicol Appl Pharmacol 173 (2): 114-119.

Wildung, M., Esser, T.U., Grausam, K.B., Wiedwald, C., Li, L., Zylla, J.L.S., et al. (2018) Transcription factor TAp73 and miRNA-449 cooperate in multiciliogenesis.

Wilhelm, M.T., Rufini, A., Wetzel, M.K., Tsuchihara, K., Inoue, S., Tomasini, R., et al. (2010) Isoform-specific p73 knockout mice reveal a novel role for delta Np73 in the DNA damage response pathway. Genes Dev 24 (6): 549-560.

Wilson, R., Roberts, D., and Cole, P. (1985) Effect of bacterial products on human ciliary function in vitro. Thorax 40 (2): 125-131.

Wloga, D., Joachimiak, E., Louka, P., and Gaertig, J. (2017) Posttranslational Modifications of Tubulin and Cilia. Cold Spring Harb Perspect Biol 9 (6).

Wodarczyk, C., Rowe, I., Chiaravalli, M., Pema, M., Qian, F., and Boletta, A. (2009) A novel mouse model reveals that polycystin-1 deficiency in ependyma and choroid plexus results in dysfunctional cilia and hydrocephalus. PLoS One 4 (9): e7137.

Wu, J., Bao, J., Kim, M., Yuan, S., Tang, C., Zheng, H., et al. (2014) Two miRNA clusters, $\mathrm{miR}-34 \mathrm{~b} / \mathrm{c}$ and miR-449, are essential for normal brain development, motile ciliogenesis, and spermatogenesis. Proc Natl Acad Sci U S A 111 (28): E2851-E2857.

Yadav, S.P., Sharma, N.K., Liu, C., Dong, L., Li, T., and Swaroop, A. (2016) Centrosomal protein $\mathrm{CP} 110$ controls maturation of the mother centriole during cilia biogenesis. Development 143 (9): 1491-1501.

Yaghi, A., and Dolovich, M.B. (2016) Airway Epithelial Cell Cilia and Obstructive Lung Disease. Cells 5 (4).

Yaghi, A., Zaman, A., Cox, G., and Dolovich, M.B. (2012) Ciliary beating is depressed in nasal cilia from chronic obstructive pulmonary disease subjects. Respir Med 106 (8): 1139-1147.

Yang, A., Walker, N., Bronson, R., Kaghad, M., Oosterwegel, M., Bonnin, J., et al. (2000) p73-deficient mice have neurological, pheromonal and inflammatory defects but lack spontaneous tumours. Nature 404 (6773): 99-103.

Yang, X., Feng, M., Jiang, X., Wu, Z., Li, Z., Aau, M., and Yu, Q. (2009) miR-449a and miR-449b are direct transcriptional targets of E2F1 and negatively regulate $p R b-E 2 F 1$ activity through a feedback loop by targeting CDK6 and CDC25A. Genes Dev. 23 (20): 2388-2393.

Yao, H., Chung, S., Hwang, J.-w., Rajendrasozhan, S., Sundar, I.K., Dean, D.A., et al. (2012) SIRT1 protects against emphysema via FOXO3-mediated reduction of premature senescence in mice. J Clin Invest 122 (6): 2032-2045. 
Yeste, M., Morató, R., Rodríguez-Gil, J.E., Bonet, S., and Prieto-Martínez, N. (2017) Aquaporins in the male reproductive tract and sperm: Functional implications and cryobiology. Reprod Domest Anim 52 Suppl 4: 12-27.

You, Y., Huang, T., Richer, E.J., Schmidt, J.-E.H., Zabner, J., Borok, Z., and Brody, S.L. (2004) Role of f-box factor foxj1 in differentiation of ciliated airway epithelial cells. Am J Physiol Lung Cell Mol Physiol 286 (4): L650-657.

You, Y., Richer, E.J., Huang, T., and Brody, S.L. (2002) Growth and differentiation of mouse tracheal epithelial cells: selection of a proliferative population. Am J Physiol Lung Cell Mol Physiol 283 (6): L1315-21.

Yuan, S., Tang, C., Zhang, Y., Wu, J., Bao, J., Zheng, H., et al. (2015) mir-34b/c and mir$449 \mathrm{a} / \mathrm{b} / \mathrm{c}$ are required for spermatogenesis, but not for the first cleavage division in mice. Biol Open 4 (2): 212-223.

Zariwala, M.A., Leigh, M.W., Ceppa, F., Kennedy, M.P., Noone, P.G., Carson, J.L., et al. (2006) Mutations of DNAl1 in Primary Ciliary Dyskinesia: Evidence of Founder Effect in a Common Mutation. Am J Respir Crit Care Med 174 (8): 858-866.

Zhang, J., Li, S., Li, L., Li, M., Guo, C., Yao, J., and Mi, S. (2015) Exosome and exosomal microRNA: trafficking, sorting, and function. Genomics Proteomics Bioinformatics 13 (1): 17-24.

Zhang, J., Wu, L., Qu, J.-m., Bai, C.-x., Merrilees, M.J., and Black, P.N. (2012) Proinflammatory phenotype of COPD fibroblasts not compatible with repair in COPD lung. J Cell Mol Med 16 (7): 1522-1532.

Zhang, K., Wang, P., Huang, S., Wang, X., Li, T., Jin, Y., et al. (2014) Different mechanism of LPS-induced calcium increase in human lung epithelial cell and microvascular endothelial cell: a cell culture study in a model for ARDS. Mol Biol Rep 41 (7): 4253-4259.

Zhang, L., Bukreyev, A., Thompson, C.I., Watson, B., Peeples, M.E., Collins, P.L., and Pickles, R.J. (2005) Infection of ciliated cells by human parainfluenza virus type 3 in an in vitro model of human airway epithelium. J Virol 79 (2): 1113-1124.

Zhang, Y., Huang, G., Shornick, L.P., Roswit, W.T., Shipley, J.M., Brody, S.L., and Holtzman, M.J. (2007) A transgenic FOXJ1-Cre system for gene inactivation in ciliated epithelial cells. Am J Respir Cell Mol Biol 36 (5): 515-519.

Zhang, Y., Kwon, S., Yamaguchi, T., Cubizolles, F., Rousseaux, S., Kneissel, M., et al. (2008) Mice lacking histone deacetylase 6 have hyperacetylated tubulin but are viable and develop normally. Mol Cell Biol 28 (5): 1688-1701.

Zhou, Q., Clarke, L., Nie, R., Carnes, K., Lai, L.W., Lien, Y.H., et al. (2001) Estrogen action and male fertility: roles of the sodium/hydrogen exchanger-3 and fluid reabsorption in reproductive tract function. Proc Natl Acad Sci U S A 98 (24): 1413214137. 